Consortium for Economic Policy Research and Advice

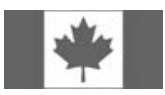

Canadian International Development Agency

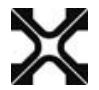

Association of Universities and Colleges of Canada
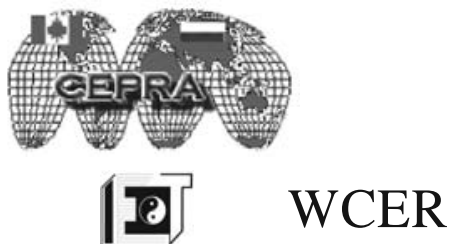

Institute for the Economy in Transition
Working Center for Economic Reform
Academy of National

Economy

\title{
Factors of Economic Growth in Russia's Regions
}


UDC 332.122(1-77)(470+571)

LBC 65.049(2Poc)+65.9(2Poc)-96

F12

Factors of Economic Growth in Russia's Regions. M.: IET, 2005. P. 268. Agency CIP RSL

Autors: S. Drobyshevsky, O. Lugovoy, E. Astafyeva, D. Polevoy, A. Kozlovskaya, P. Trunin, L. Lederman.

The monograph deals with analysis of the factors that determine the diversity in the degree and pace of economic development of Russia's regions. In particular, basing on the data of main socio-economic indicators of RF constituent regions' performance, the authors have empirically tested various convergence concepts and attempted to conduct decomposition of economic growth and assess the dynamics of labor productivity in Russia's regions. One of the sections of the monograph focuses on the Canadian record of development of individual regions and equalization policies. The monograph contains conclusions and recommendations for development of the federal economic policy with respect to different Russian regions.

JEL Classification: O18, O47, R11

Editors: Glavatskaya N., Mezentseva K., Serianova S.

Prof-reader: Andrianova N.

Page setting: Yudichev V.

The research and the publication were undertaken in the framework of CEPRA (Consortium for Economic Policy, Research and Advice) project funded by the Canadian Agency for International Development (CIDA).

ISBN 5-93255-180-1

5, Gazetny per., Moscow, 125993 Russia

Tel. (495) 629-67-36, Fax (495) 203-88-16

E-mail - info@iet.ru, Web Site - http://www.iet.ru 


\section{Contents}

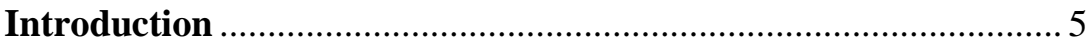

1. Theoretical Fundamentals of Analysis

of Regional Growth ........................................................................ 8

1.1. Issues of Growth in the Theory

of Regional Economy....................................................................... 8

1.1.1. Theory of Growth in Regional Economics ...................... 9

1.1.2. Agglomerations Theory .................................................. 13

1.1.3. "Nucleus-Periphery” Models ............................................ 15

1.1.4. The Random Growth Theory........................................... 18

1.1.5. The Role of Innovations in Differences

in Regional Growth .................................................................. 20

1.2. Issues of Regional Development in Russian

Economic Literature....................................................................... 22

2. The Concept of Convergence and Its Application

to the Evaluation of Economic Growth in the RF Regions............ 30

2.1. The Convergence Theory ........................................................... 30

2.2. An Empirical Testing of Convergence

Concepts in Respect to the RF Regions ............................................. 45

2.2.1. Comparative Analysis of the RF Regions

by GRP over the Period between 1994

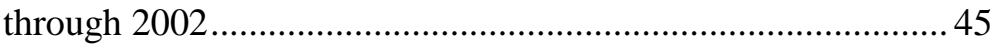

2.2.2. Testing Hypothesis of $\sigma$-Convergence............................ 49

2.2.3. Testing the Concept of the Unconventional

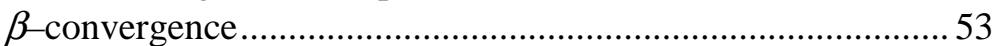

2.2.4. The Impact of Budget Policy

on Convergence between Russia’s Regions:

Testing the Hypothesis of Conventional

$\beta$-convergence.......................................................................... 54

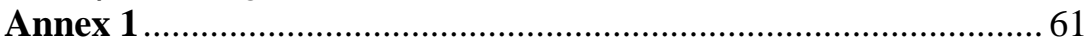

3. Decomposition of Economic Growth

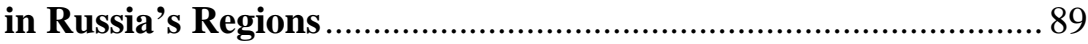

3.1. Methodology and Data ............................................................ 90 
3.2. The Main Approach to Decomposition

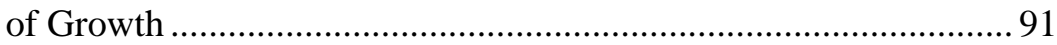

3.2.1. Assessing Output .............................................................. 94

3.2.2. Assessing Labor Input ...................................................... 101

3.2.3. Assessing Capital Input ................................................. 110

3.2.4. Assessing Weight Coefficients

to Integrate Inputs .................................................................. 122

3.2.5. Decomposition of Growth ............................................... 125

3.3. The Dual Approach to Decomposition

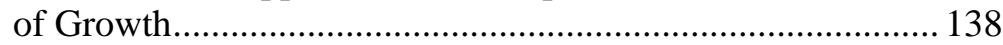

3.3.1. Comparing Assessments Made

by Different Methods................................................................. 142

3.4. Labor Productivity in the Industrial Sector ............................ 147

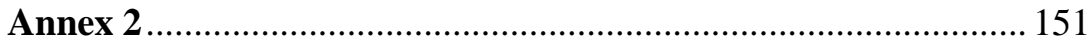

\section{Economic Growth in Regions. Relevant}

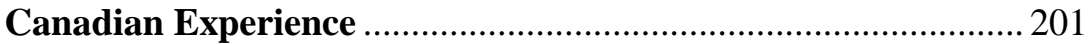

4.1. Capital Imports -Human and Financial ................................. 202

4.2. Federal "National Policy" ........................................................ 203

4.3. US-Focus - Reciprocity / Free Trade / Continentalism .......... 203

4.4. Resource-based Growth: Extraction and Processing .............. 204

4.5. Trade-based Growth ............................................................. 207

4.6. Finance-based Growth .......................................................... 208

4.7. Shifts from Region to Region.................................................. 216

4.8. Focus on "Disparities" .............................................................. 217

4.9. The Federal Spending Power: Scope and Limitations ........... 229

4.10. A New Approach to Regional Development

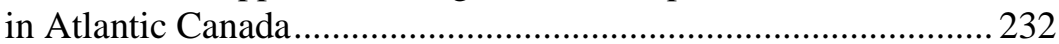

4.11. Infrastructure Grants and Agglomeration Economies.......... 238

4.12. Prospects of the Development of Atlantic Canada................ 244

\section{Main Conclusions and Economic}

Policy Recommendations .......................................................... 254

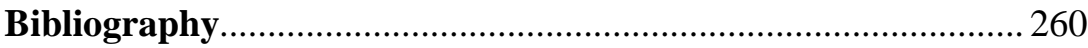




\section{Introduction}

The past five years starting from 1999 saw a rapid growth in Russia's economy. At the time, in the aftermath of the 1998 crisis and the four-fold depreciation of the Ruble, import substitution processes in the domestic market have triggered the growth. Later a favorable state of affairs on external markets and the unfolding recovery growth of the economy supported the growth, while over the past two years it is the expansion of the domestic demand for all the spectrum of goods whose contribution to the GDP growth rates was increasing.

Whilst on the macrolevel the main factors of economic growth are more or less evident and, to some extent, studied into (see, for instance: Entov, Lugovoy at al. (2003)), there practically is no analysis of processes on the level of Russia's single regions ${ }^{1}$. It is evident though, that both at the stage of transformational slump and the one of economic growth dynamics of main indicators that characterize regional economies appears very diverse. This causes a serious differentiation between the RF Subjects in terms of their well-being and economic development capacity. The differentiation intensifies because of differences between them in terms of climatic and natural conditions, abundance or scarcity with minerals, etc.

In the frame of CEPRA, there have been some papers that dealt with single issues directly associated with growth rates and its differentiation across the RF Subjects, albeit the present research does not focus on fundamental causes of these processes. More specifically, the paper “Typology of Russian Regions"2 presented a typol-

\footnotetext{
${ }^{1}$ The review of papers on various aspects of regional development in RF is given in section 1.2.

${ }^{2}$ Buts, Drobyshevsky at al.(2002).
} 
ogy of the RF Subjects received on the basis of a qualitative analysis and combination of economic capacity, living standards and investment activity indices.

The paper entitled "Investment: the Regional Aspect"3 demonstrated that in 1992-2001 investment processes had been very different in different RF regions. The differences can be attributed both to then existing level of development of single industries and regions' profiles under the Soviet planned economy and the geographical and sectoral structure of foreign investments. The latter found themselves under the impact of primarily the level of maturity of market relations in a given sector or Subject of RF, as well as other institutional and political factors.

The present project focuses on an analysis of factors that determine differences in the extent and rate of economic development of Russian regions. More specifically, we have decided to center on researching into convergence between Russia's regions in terms of their social-economic development indicators. As well the research focuses on singling out components (alias the contribution of particular factors) of economic growth in the medium run.

The paper comprises four chapters and Conclusion. The first chapter provides a review of fundamental provisions and issues of the theory of regional economy that concern issues of regional growth and regional economic policies, among others. As well, the chapter contains a review of main papers on regional development published in Russia. The second chapter deals with the theory and main types of convergence, and empirical testing of various concepts of convergence. The testing is based on data of main socioeconomic performance indices of Russia's Subjects.

\footnotetext{
${ }^{3}$ Dneprovskaya, Drobyshevsky at al. (2002).
} 
The third chapter forms the first attempt to decompose (break into components) economic growth in Russia's regions and to assess differences in total factor productivity in the RF Subjects.

The fourth chapter highlights on Canada's experiences in developing single regions and in the area of equalization.

Finally, the Conclusion provides economic policy recommendations. 


\section{Theoretical Fundamentals of Analysis of Regional Growth}

\subsection{Issues of Growth in the Theory of Regional Economy}

Regional economics ${ }^{4}$ constitutes a part of the economic theory that deals with differentiation and interrelation between various parts of a space in the condition of unevenly dispersed and imperfectly mobile resources. It focuses particularly on planning investment to smooth down various social problems. Regional economy originates from the theory of spatial interactions that in turn deals with studies into firms' decisions on location of production and selection of a size and geographical position of markets. The task of placement of production operations in the space is closely linked to the problem of identification of an optimal output, technology and location of production, and the selection of a pricing mechanism.

An analysis of placement as a part of the regional theory shed the light on causes of emergence of certain kinds of production in different regions. However, the placement theory fails to provide an answer to more global questions that concern regional differences within regions. More specifically, why some regions see a steady rise in production operations, while other witness it fade gradually? Region's uneven development may become a consequence of differences in their geographical, social and economic positions. Different models of regional growth allow singling out factors that either encourage, or inhibit growth in some regions visà-vis others. The difference between regional models from traditional models of economic growth, as a rule, lies with the fact that the regional approach requires building multidimensional models, which can considerably complicate the receiving of certain conclu-

\footnotetext{
${ }^{4}$ See: Nijkamp (1986).
} 
sions. In some cases, multiregional models are reduced to a modeling of two regions: that is, a backward South and a more developed North.

\subsubsection{Theory of Growth in Regional Economics}

The neoclassical approach to modeling of regional growth considers regions as production units, a equilibrium between which can be achieved by means of markets. The first neoclassical models explain an uneven development of regions with temporary deviations from the equilibrium or an imperfect adjustment in response to exogenous shocks. So, the differentiation should disappear in the long run. More advanced neoclassical models consider such phenomena as growing economies of scale and cutting-edge technologies, investments, and dynamic aspects of the models. While the neoclassical approach can be suitable for researching into regional effects, it suffers certain drawbacks, such as, for instance, overly strict prerequisites used to explain regional growth, an excessive focus on the processing sector and a relative ignorance of social and political factors ${ }^{5}$.

Harrod (1948) provides the simplest regional model of economic growth, albeit his conclusions can be applied to a closed economy isolated from the external world. To consider the openness of a given region, one may consider its economic and trade relations with the external world, assuming at the same time that parameters of the latter are preset exogenously, while the region in question is small enough to exert influence on the global parameters, such as, for instance, interest rates. The research into interregional relations may be carried out in the frame of a model that implies two and more regions. The most pictorial form of a modeling of interactions between two small regions may be represented by a

${ }^{5}$ Nijkamp (1986), p. 654. 
model of two identical regions whose dynamics is described by a system of equations as follows:

$$
\begin{aligned}
& Z_{i}=x_{i}+m_{i} x_{i} ; \\
& C_{i}=\alpha_{i} x_{i} ; \\
& I_{i}=\beta_{i} x_{i}^{\prime},
\end{aligned}
$$

where $x_{i}$ - output in region i, i=1,2; $m_{i} x_{i}-$ import in region $\mathrm{i}, Z_{i}, C_{i}$, $I_{i}$ - aggregate offer, demand and investment in region i, respectively.

In this model, the condition of an interregional equilibrium would suggest observance with the following conditions that are called the interregional equilibrium growth conditions:

$$
\begin{aligned}
& x_{1}+m_{1} x_{1}=\alpha_{1} x_{1}+\beta_{1} x_{1}{ }^{\prime}+m_{2} x_{2}, \\
& x_{2}+m_{2} x_{2}=\alpha_{2} x_{2}+\beta_{2} x_{2}{ }^{\prime}+m_{1} x_{1},
\end{aligned}
$$

that imply that the produce is spent on consumption, investment and net export from region $i, m_{i} x_{i}-m_{-i} X_{-i}$.

In the case there exist such solutions to system (1.1) that

$$
\dot{x}_{i}=\lambda x_{i}, i=1,2,
$$

one argues then that in a given economy, there exists a balanced growth equilibrium when growth in both regions is proportional in the long run.

If system (1.1) is given in the form

$$
\begin{aligned}
& \dot{x}_{1}=G_{11} X_{1}-G_{12} X_{2}, \\
& \dot{x}_{2}=G_{21} X_{1}-G_{22} X_{2},
\end{aligned}
$$

then an exponential growth less oscillations will be noted provided the correlation below is met $\left(G_{11}+G_{22}\right)^{2}>4\left(G_{11} G_{22}-G_{21} G_{12}\right)$. 
For identical regions, this condition is determined by parameters Для идентичных регионов это условие определяется параметрами $m_{1}$ and $\beta_{2}$, in accordance with the expression $m_{1} / \beta_{2}>0$,

Which is always valid fro two regions, providing the absence of autarchy. On the qualitative level, it is possible to identify parameters of the balanced growth by means of a graphical illustration (see fig. 1.1), where $H(y)=G_{11}-G_{12} y^{-1} ; F(y)=G_{22}-G_{21} y, Y=x_{1} /\left(1-x_{1}\right)$.

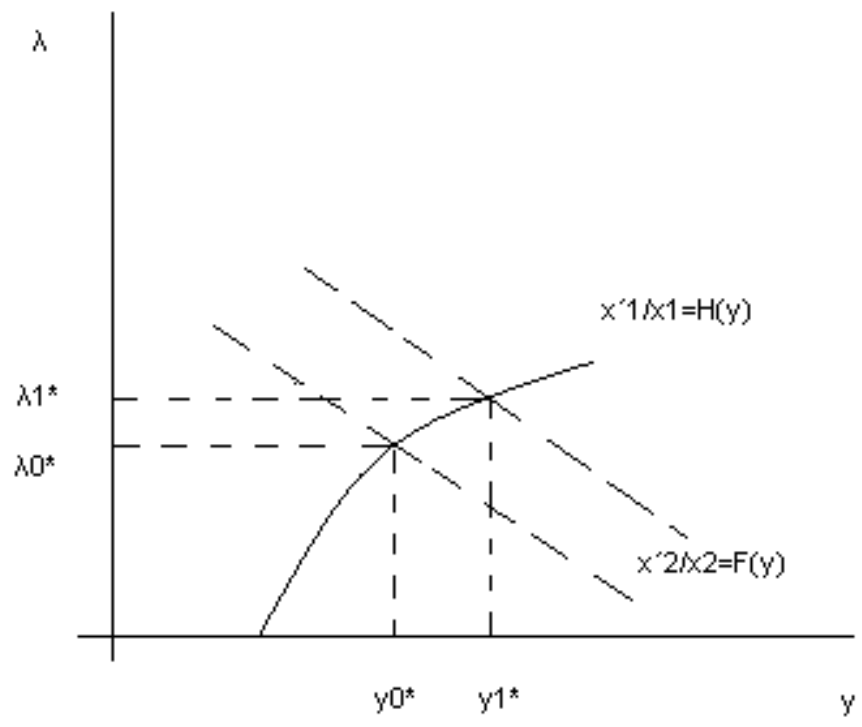

Fig. 1.1. Parameters of balanced growth on the qualitative level

As seen from fig. 1.1, in the event the inclination to consumption in region $2\left(\alpha_{2}\right)$ falls, $G_{22}$ and $F(y)$ demonstrate growth. Because of the rise of $\alpha_{2}$, the parameter of balanced growth surges, as the share of region 1 in the total output does. Only in the event both regions equally increase their inclination to saving will their equilibrium growth rates surge in both regions, while the structure of output between them will remain unchanged. 
The framework of the neoclassical analysis of growth suggests a less trivial model for the final number $n$ of regions. In the absence of depreciation and savings of a domestic output and net imported offer, the dynamics of capital in each region will be determined by the following expression:

$$
K_{r}{ }^{\prime}=\sigma_{r}\left(Q_{r}-E_{r}+M_{r}\right) \text {, }
$$

where $K_{r}^{\prime}$ - an instant change of capital in region $r, \sigma_{r}$ - inclination to savings in region $r, Q_{r}-$ GRP in region $r, E_{r}, M_{r}$ - export and import in region $r$.

Once we assume that the share of export in each region's output accounts for $\varepsilon_{r}$, given that $\sigma_{r}>\varepsilon_{r}>0$, the model of growth of both regions will be then depicted by the following system of equations:

$$
\begin{aligned}
& K_{1}{ }^{\prime}=\left(\sigma_{1}-\varepsilon_{1}\right) Q_{1}+\varepsilon_{2} Q_{2}, \\
& K_{2}{ }^{\prime}=\varepsilon_{1} Q_{1}+\left(\sigma_{2}-\varepsilon_{2}\right) Q_{2} .
\end{aligned}
$$

The above models are based upon an analysis of value added and do not take into account differences in the production structure and possible associated effects. For the purpose of evaluation the interregional development, the interdependence of regional output in the models can be considered as follows: let us assume that xijflow of goods from region $i$ into region $j$. The dynamics of flows of goods for each region and sector will consequently be defined with the following equations:

$$
\begin{aligned}
& x_{i}^{r} \geq \sum_{s j} a_{i j}^{r s} X_{j}^{s}+\sum_{s j} b_{i j}^{r s} X_{j}^{s}, \\
& r=1, \ldots, R ; i=1, \ldots, N ;
\end{aligned}
$$

where $x_{i}^{r}$ - an aggregate production of good i in region $r$; $a_{i j}{ }^{r s}-$ regional coefficients of production costs; $b_{i j}{ }^{r s}$ - coefficient of investment costs of sector $i$ per unit of output in sector $j$. 
The balanced growth equilibrium, i.e. the equilibrium for which condition (1.2) I observed, implies the observance with the correlation:

$$
x_{i}^{r}=\sum_{s j} a_{i j}^{r s} x_{j}^{s}+\lambda \sum_{s j} b_{i j}^{r s} \dot{x}_{j}^{s},
$$

where $x_{i}^{r}$ - production of good $\mathrm{i}$ in region $\mathrm{r}, b_{i j}{ }^{r s}$ - correlation between marginal capital and output $\left(=I_{i j}{ }^{r s} / x_{j}^{S}\right), \lambda$ - total coefficient of growth for which $\lambda x_{i}^{r}=x_{i}^{\prime r}$.

If the production of good $x_{i}{ }^{r}$ is determined by the Cobb-Douglas technology, i.e.

$$
\ln x_{i}^{r}=\ln a_{i}^{r}+\sum_{s j} \alpha_{j i}^{s r} \ln x_{j i}^{s r},\left(\sum \alpha_{j i}^{s r} \leq 1\right),
$$

where $x_{j i}^{s r}$ - factor $j$ imported from region $s$ for the production of good $i$ in region $r, a_{i}^{r}, \alpha_{j i}{ }^{s r}$ - parameters of the technology,

then the solution to the task of minimization of production costs under a preset level of output with account of production function (1.3) allows to show that all interregional coefficients of output costs form functions of only prices and transportation costs.

\subsubsection{Agglomerations Theory}

An uneven distribution of production under equilibrium leads to the emergence of agglomerations. Their unfolding is attributed to a random factor or to the notions of increasing economies of scale ${ }^{6}$. According to the theory, production activity concentrates in certain regions, because firms benefit from their expansion or positive externalities that emerge due to the presence of other firms on the market. The causes for the increasing economies of scale can vary. For instance, they may result from a flow of knowledge, merger of labor markets, or due to the economy arising because of shorter dis-

\footnotetext{
${ }^{6}$ Krugman (1991), Romer (1992).
} 
tances between producers and consumers in the conditions of a trade that require costs.

Fiani (1984) suggests a model of the economies of two regions (North and South), wherein the existence of an increasing return in the production from a non-tradable factor (services) entails a rise in differences between the regions' growth rates. The model suggests that initially the regions are identical, i.e. they enjoy the access to identical technologies. As far as tradable goods are concerned, the production is described by function $F[\cdot]$ :

$Q_{T}=F\left[\operatorname{Val}\left(L_{T}, K_{T}\right), Q_{N}\right]$,

where $Q_{T}$ - output of a given tradable, $\operatorname{Val}(\cdot)$ - value added as a labor and capital function, $L_{T}, K_{T}, Q_{N}$ - production factors (labor, capital and a non-tradable intermediate factor).

The market for non-tradables is monopolistically competitive, and the output therein is driven by function $G(\cdot)$ that bears a constant elasticity by production factors:

$Q_{N}=G\left(L_{N}, K_{N}\right)$,

where $Q_{N}$ - output of the non-tradable, $L_{N}, K_{N}$ - production factors.

The increasing economies of scale in this model arises due to the fact that the market for non-tradables is represented by monopolistically competitive producers that face identical demand curves.

The prices for the non-tradables are computed according to the rule

$P_{N}=w a_{N}(1+q)$,

where $w$ - wages, $a_{N}$ - olabor to capital ratio, $q$ - the monopoly's extra.

In this model, a special attention is paid to export and investment in regions with different development levels. Whilst saving in one region can be invested in the other, this, with a due account of 
costs associated with establishment of investments, would allow to fulfill the following balance correlation:

$r^{N} K^{N}+r^{S} K^{S}=I^{N}+V\left(I^{N}\right)+I^{S}+V\left(I^{S}\right)$,

where $V(\cdot)$ - function of investment costs.

It can be demonstrated that under static expectations, the effective discounted marginal investment in region i will be computed according to the formula

$R^{j} / i-V^{\prime}\left(I^{j}\right)-p_{T}=\pi^{j}$,

where $i$ - the total discount rate.

While studying into dynamics of the model, its authors showed that the increasing economies of scale entail divergence of growth rates between different regions within a given economy. The expansion of the model by adding the third region allowed to demonstrate that each region would be keen to center on the production of a sole tradable. The authors showed that even in the assumptions of a cost-free production and instant inter-regional capital flows there would be noted a specialization in production of a given good, which would prove to be more intense in terms of a non-tradable factor (services) in the North. By contrast, the South would see a specialization of the production of a good, which will be less intense in the above terms. Accordingly, the authors assume that a high level of substitution between capital and service factors necessitates encouragement of investment in the Southern region, with the greatest emphasis to be put on sectors with low demand for service factors and, accordingly, a low impact of the mutliplier on the local economy.

\subsection{3. "Nucleus-Periphery” Models}

It was Murdal (1957) and Hirschman (1958) that laid down and pioneered studies into these particular models. Murdal's analysis 
implied that the start of a given region's development is related to a random factor, such as, for instance, a discovery of mineral deposits or development of the exportation of food stuffs. A rise in real wages coupled with a high return on capital generate increasing economies of scale and give a rise to a spatial external economy agglomeration that manifests itself in the growing productivity of labor and capital as the function of the regional output growth rate.

Ottavanio and al. (2002) consider various aspects of agglomeration and trade by using the "nucleus-periphery" model. The authors show that their model can be used particularly to study the welfare effect associated with the rise of agglomerations. Their model suggests there exist two regions $\mathrm{H}$ и $\mathrm{F}$ and two factors $\mathrm{A}$ и $\mathrm{L}$. The immobile factor $\mathrm{A}$, which is farmers, is distributed evenly between the two regions. $\lambda$ - the proportion of mobile factor $L$ in region $H$. It is consequently assumed that there exist two kinds of goods in the economy - homogenous $\left(\mathrm{q}_{0}\right)$ and differentiated $\left(\mathrm{q}_{\mathrm{i}}\right)^{7}$.

Under this model individuals' preferences are identical and can be desribed by a quasi-linear utility function with quadratic correlations that take the following form:

$$
U\left(q_{0} ; q(i), i \in[0, N]\right)=\alpha \int_{0}^{N} q(i) d i-\frac{\beta-\gamma}{2} \int_{0}^{N}[q(i)]^{2} d i-\frac{\gamma}{2}\left[\int_{0}^{N} q(i) d i\right]^{2}+q_{0} ;
$$

where q(i) - amount of differentiated good $i, i \in[0, N] ; \alpha>0$ - parameter that characterize the level of preference of the differentiated product; $\beta, \gamma$-utility parameters. Notably, with the preset $\beta, \gamma$ characterizes the substitution feature between differentiated goods.

It is assumed that consumer are more apt to consume a differentiated product, i.e. $\beta>\gamma>0$.

An individual's budget constraint takes the form: ${ }^{7}$ It is assumed that the market for the differentiated good is monopolistically
competitive. 
$\int_{0}^{N} p(i) q(i) d i+q_{0}=y+\bar{q}_{0}$,

where $y$ - the individual's labor income, $\bar{q}_{0}$ - initial allotment with a good of the first type (it price is standardized by unit).

Production is represented by the number of firms $n_{H}$ and $n_{F}$ in regions $H$ and $F$ respectively, while their number equals the number pf differentiated products $N$. The authors show that a firm's profit in region $H$ can be presented as follows:

$\Pi_{H}=p_{H H} q_{H H}\left(p_{H H}\right)(A / 2+\lambda L)+\left(p_{H F}-\tau\right) q_{H F}\left(p_{H F}\right)[A / 2+(1-\lambda) L]-\phi w_{H}$,

where $p_{H H}$ and $q_{H H}$ - price of, and demand of an individual in region $H$ for an offer of a firm residing in region $H$, respectively, while $p_{H F}$ and $q_{H F}$ - price of, and demand of an individual in region $H$ for an offer of a firm residing in region $F$ respectively, $\phi-$ parameter that characterizes a reverse of the mas of firms, and $\tau-$ trade costs.

The demand is set by the following formulas:

$q_{H H}=a-(b+c N) p_{H H}+c P_{H}$,

$q_{H F}=a-(b+c N) p_{H F}+c P_{F}$.

$P_{H}=n_{H} p_{H H}+n_{F} p_{F H}$

$P_{F}=n_{H} p_{H F}+n_{F} p_{F F}$.

In the equilibrium, prices are determined by the condition of maximization of the firms' profits, while equilibrium wages are set by the condition of zero profit as an effect of a free entrance to and exit from the market. The authors show that equilibrium prices depend on demand and distribution of firms between regions. More specifically, with the mass of local firms growing, both local and foreign firms ${ }^{8}$ prices fall, while this downfall appears the lesser,

\footnotetext{
${ }^{8}$ In the model, this is conditioned by the drop in trade costs.
} 
the smaller $\tau$ is. Within the limits ${ }^{9}$ the firms' moving to region $H$ will not affect prices. They also show that with the rise in the amount of the mobile factor, a firm located in region $H$ will see its profit on the one hand, decline, which can be explained to an intensifying competition, and grow on the other, as the number of consumers in the given region is growing. So an ultimate effect remains uncertain.

The most important result of Ottavanio et al.'s model (2002) lies in the proof of stability of conclusions in relation to the selection of a specification of the model. More specifically, alternative assumptions in respect to preferences and transportation costs do not modify main conclusions, albeit the latter may change, providing a drastic change of the prerequisites.

While criticizing the "nucleus-periphery" approach, Gilbert and Gagler (1982) argue that such models underestimate the role of international influence and pay insufficient attention to social regional aspects, such as poverty and income differentiation. As well, they these models do not consider the pre-colonial history of nations and groundlessly introduce an assumption of a government acting on the population's behalf.

\subsubsection{The Random Growth Theory}

This particular theory forms an alternative explanation to the emergence of agglomerations. According to the theory, the latter emerges thanks to strong random shocks which provide impetus to the development of the economic activity in a given region. While employing a model of selection of location by plants, Allison and Glazer (1997) demonstrated that even if the plants are randomly dispersed in a given space and there exist no geographical preferences, the concentration of an industry will emerge at random.

\footnotetext{
${ }^{9}$ When $\tau$ is infinitely small.
} 
More than that, this process will lead to a positive correlation between the average size of the plant and concentration of the industry. The latter and the plants' average size will be growing over time in the location of a very huge plant.

Holmes (1999) argues that the enterprise's size should negatively correlate with the concentration of industrial activity, as the emergence of an expanded intermediary goods offer network in production concentration zones should encourage the rise of incentives to establish small, narrow-profile plants. Holmes and Stevens (2002) demonstrated that the plants' size on the whole grows in parallel with the rise in the concentration of production activity in all the sectors, except for the textiles. To explain the latter phenomenon, they argue that plants located in the zones of concentration of production activity wins because of production benefits visà-vis those located beyond such zones. That is why the former boost their size to capitalize on the benefits. Such benefits may take their roots in geographical differences or in the agglomeration benefits.

In their empirical study, Davis and Wainstein (2002) attempt to explain the distribution of economic activity within a single country by testing a model of increasing return from economies of scale along with two other theories, that is, the random growth theory and the location theory using Japanese cities as an example. The authors have arrived to the conclusion that the location theory could explain differences in regional concentration of economic activity, while the theory of increasing return from economies of scale was responsible for the level of a spatial differentiation of economic activity. The random growth theory has failed to find a proof against such a background. 


\subsubsection{The Role of Innovations in Differences in Regional Growth}

Numerous regional development models pay a great attention to innovational factor as a main cause of differences between regions' development level. While studying into factors of regional development, Andersen and Mancinen (1981) employed the theory of knowledge creation and diffusion. The authors model regions with the neoclassical production function $Q_{r}=F_{r}\left(C_{r}, a_{r}\right)^{10}$,

where $C_{r}$ - amount of capital in region $r, a_{r}$ - availability of knowledge in region $r$, is found according to formula $a_{r}=\sum_{s} \exp \{-$ $\left.\beta d_{r s}\right\} K_{s}$, while $d_{r s}$ - distance between regions $r$ and $s, K_{s}$ - accumulated amount of knowledge.

The model suggests that capital and knowledge grow in proportion to the aptness to savings $\sigma_{r}$, while the distribution between share of investment in physical capital and $R \& D$ is determined by the share of the latter in the total capital accumulation:

$$
\begin{aligned}
& C_{r}=\left(1-\rho_{r}\right) \sigma_{r} F_{r}\left(C_{r}, \sum_{s} e^{-\beta d_{r s}} K_{s}\right) \\
& K_{r}=H_{r}\left(K_{r}, \rho_{r} \sigma_{r} F_{r}\left(C_{r}, \sum_{s} e^{-\beta d_{r s}} K_{s}\right)\right), r=1, \ldots, n
\end{aligned}
$$

where $H_{r}$ - positive, smooth functions, found in $R_{+}$.

The authors demonstrated that this system sees an equilibrium for which the capital to knowledge ratio is constant. The analysis of dynamics of the system allows to show that with any distance $d_{r s}$ reducing, the growth rate for all the regions rises in equilibrium. In the frame of assumptions of increasing return from economies of scale and the existence of spatial frictions, the model in question predicts that the bias of the R\&D share in the total capital towards

\footnotetext{
${ }^{10}$ It is assumed that the use of labor is proportional to capital and it is exogenously determined by the output offer.
} 
values higher than an equilibrium value may lead to an initial short-term rise in the regional income inequality.

$$
* * *
$$

Hence the regional growth theory as a whole is based on the prerequisites that the neoclassical theory of growth in macroeconomics that prevails nowadays. The distinctive feature of the regional growth theory lies with its greater emphasis put on issues of unevenness of growth in neighboring economies (regions). It should be noted that the macroeconomic growth theory practically ignores the external world and assumes availability of any foreign trade balance that combines with a sustained domestic growth.

Whereas the regional economy theory implies that a given region may not be considered in isolation from other regions of an integral national economy, it provides for certain constraints associated with a possible unevenness of growth and differences in initial conditions. To consider such constraints new models were designed, particularly, agglomeration, "nucleus-periphery" and random growth ones.

So, the above review of regional economy theories that deal with regional growth issues allows to fundamental conclusions that appear necessary for carrying out further research into growth in Russia's regions:

1. The neoclassical growth theory and, accordingly, approaches to its empirical evaluation appear applicable to an analysis of factors and structure of growth in single regions;

2. Before researching into growth factors at the regional level, it is necessary to analyze the nature of regions' uneven development rates, i.e. to test the hypothesis on convergence. 


\subsection{Issues of Regional Development in Russian Economic Literature}

Between 1997 through 2004 the Russian economic literature provided a number of research outputs albeit a very few ones, that concerned issues of economic growth in Russia's regions. For instance, Pchelintzev (2001a) studied economic growth in RF between 1999-2001 and argued that the recovery growth had failed to overcome the inertia of the preceding crisis development, for that necessitated re-switching the national economy into the investment growth mode. The author argued that it had not happened at that moment. The paper provides an analysis of regional development under economic growth.

The author stresses that in the period in question growth rates in the areas of processing sectors for the first time proved to be greater than in the areas of mineral extraction and primary processing. He consequently draws a conclusion that the range of the "slump-rise" amplitude proved to be smaller in the regions that had succeeded in integrating themselves into the global market. He also notes that in 2000 interregional differences in growth rates diminished notably, with the regions of the processing sector being leaders in terms of growth rates. That has somewhat smoothed down disproportions that had arisen between them and the "mineral" areas back in 1992-1998.

The author's analysis allows the conclusion that despite the emergence of some new phenomena, the main trends of regional development in Russia in 1999-2000 remained the same:

- a passive adjustment of the territorial-sectoral structure inherited from the Soviet time to the global market's demands;

- degradation of the processing sector areas that had been forming the core of the national economy over nearly a century;

- preservation, and in some cases, intensification of the interregional differentiation; 
- emergence of a new spatial hierarchy that is based upon the predomination of a small number of large trading and intermediary centers and regions centering on mineral exports.

So, the paper argues that while the economic growth of 19992000 have cushioned some manifestations of the systemic crisis, it has failed to overcome its inertia. More than that, since the second half 2000 industrial growth in the country once again has been followed by stagnation.

Lavrovsky (1999) attempted to measure a regional asymmetry in RF. The researcher understands asymmetric development as such a type of regional development over a certain period of time under which regions that enjoy a relative advantage by a specific indicator at the beginning of the period increase it further on, while those relatively backward regions further aggravate it. Understandably, the symmetric type of development implies that a gap between levels of regions' indicators shortens. The author's analysis of asymmetry of regions' development in terms of the indicator of economic growth is based on the following assumptions:

1. Regional development is uneven, and the dynamics of dispersion of regional indicators constitute the main manifestation of asymmetric development;

2. The type of regional development can be found only in the dynamics and only in respect to a specific indicator;

3. Asymmetric development entails an intensification of centrifugal tendencies, regions' respective indicators distancing from each other, and an intensification of interregional differences, while symmetric development leads to convergence of the regions indicators.

To measure an asymmetry, the author employs the coefficient of variation of the respective indicator. He argues that the process of regional differentiation of major economic indicators in early 1990s was very intense, while the production reduction was uneven across 
the country. However, up to the mid-1990s systemic nationwide development factors predominated over local ones, i.e. the fall in industrial output practically had no regional borders. The situation changed shortly afterwards, and as early as in 1997 Russia's regions split practically into two halves in terms of the sign of production increment. The paper consequently concludes that as far as a parameter of economic growth is concerned, the type regional development in Russia in the 1990s was unambiguously asymmetric. Plus, the author's evaluation of the dynamics of differentiation of regions by a number of macroeconomic indicators allowed him to argue that while a decline is accompanied by regional disparity, a growth goes hand in hand with regional equalization processes.

Seleznev (2002) conducts an evaluation of "constraints to economic growth that are objectively determined by the state of Russia's economy the account of which is of a substantial importance to justification for scenarios of economic development". The author particularly focuses on limited possibilities for selecting optional scenarios. Such possibilities are preset by the inertial nature of the economy and by reforms of the 1990s. The author also analyzes the regional structure of GDP from the perspective of intensity of negative processes in Russia's economy that require a "new" strategy of growth.

The paper particularly stresses that the structure of GRP provides a comprehensive picture of recipients of, and donors to the federal budget. The author believes that such a structure evidences the bankruptcy of concepts of regions "self-reproduction" in conjunction with their natural, climatic and other parameters. The differentiation between regions' socio-economic development levels accentuates an urgent need in a centralized administration of investment resource flows and expansion of the government entrepreneurial activity. 
The author at the same time argues that progress in the municipal sector constitutes a substantial factor of galvanizing economic growth. However, it is impossible to mobilize this particular sector's potential, if it finds itself isolated from the government vertical of power and providing there exists a fully unsound downward arrangement of budgetary flows that implies an absolute pendency of the issue of procedures of the centralized allocations of investment funds with account of the municipal sector's interests and capacity.

Basing on his analysis of regional development tendencies in the 1990s, Pchelintzev (2001b) advocates the need for shaping an economic system of sustained development (reproductive economy) and considers some, mostly foreign trade and social, conditions of a transition towards sustained development.

The paper states that Russia's regional development takes place in the frame of the economic model established in 1992-94 resulting from the liberalization of a non-equilibrium economy Russia had inherited from the USSR. The author argues that at first winners (of course relative to the overall slum) were areas that supplied minerals and fuel for export, while none of the old industrial centers (such as Moscow, St. Petersburg or Nizhny Novgorod), the home for the processing sector, no longer is in the top echelon of industrial areas. Accordingly, the mere concept of old industrial region has changed, and the coal and metallurgical areas have given this role to centers of the processing sector, research and technology areas.

The author suggests that this problematic situation can be resolved only providing a drastic change of the economic development strategy, which in a nutshell can be defined as a transition towards sustained development. He views this concept as an alternative to both the actual polarized global development underway and the doctrine of globalization. All this, the author argues dictates the 
need for the transition from the current resource-utilizing economy to the economy of their systemic reproduction. He believes that it is regions that should form the core engine of such a transition, and they should become subjects of the sustained development. This particular mission is explained by their objective role of arena for the reproduction (the room for the interaction between resource subsystems) and the presence of a series of subjective preconditions, such as territorial development experiences and the possibility for a broad application of the science of resource cycles and territorial-production complexes. The paper specifically accentuates that regions' territorial and human capital development efforts would be futile, unless they fail to meet some external (relative to regions) conditions of sustained development.

Finally, the paper by $N$. Smorodinskaya, A. Kapustin and V. Malygin (1999) studies results of development of Kaliningrad Oblast as a free economic zone in 1994-98. The paper shows that by its macroeconomic indicators the Oblast was one of the least prosperous regions in RF, while along with a general trend of contraction in output volumes in all the sectors of its economy, there existed considerable differences in annual decline rates across the sectors. Accordingly, the Oblast's specialization in the nationwide division of labor changed.

The authors conclude that so far as a complete opening of the region's economy has failed to ensure structural impulses to stabilize the economy and renew the growth. There were a number of reasons underlying this situation. First, the Oblast originally was one of the regions whose perverse sectoral production structure has proved to be most exposed to market competition forces. Second, while foreign trade benefits helped to solve the problem of food supplies to the Oblast by means of import, they nonetheless have failed to diminish the transportation component in production costs. Third, the actually appreciated Ruble exchange rate has made im- 
port operations in the Oblast much more profitable vis-à-vis the rest of the "mainland". As a result, instead of creating comparative competitive advantages for companies of the real sector and normalizing living standards of most local residents, the free trade regime has entailed an accumulation of additional deformations in the economic structure of the region.

In addition to the noted papers in leading Russian economic journals, we would like to specifically note the monograph "Valovoy regionalny product: mezhregionalnye sravneniya I dinamika" ("Gross regional product: the interregional comparisons and dynamics”) by A. Granberg and Yu. Zaitseva ${ }^{11}$ and the paper by N. Mikheeva $^{12}$.

In their monograph, A. Granberg and Yu. Zaitseva provide a detailed analysis of the methodology of accounting single components of gross regional product both in static and dynamic terms. They particularly suggest methodological approaches to adjustment of growss regional product on the basis of consideration of interregional differences in prices and the Ruble purchasing power. The paper evaluates the redistribution of GRP between Russia's regions in the system "region - country - the world" (basing on the 1999 data). The authors have ultimately developed adjusted dynamic GRP series over the period 1996-2001 and built a classification (ranking) of the regions by their growth rates and contribution to Russia's GDP. It should be noted, however, that while the research was built upon the evaluation of the final data on the volume and dynamics of GRP and its components, it ignored factors that underlie the structure and growth rates of a regional product.

N. Mikheeva studied issues of differentiation of indices of the Russian regions' socio-economic positions in 1990-96. The author assessed volumes of gross regional products in constant prices and

\footnotetext{
${ }^{11}$ Granberg, Zaitseva (2003).

${ }^{12}$ Mikheeeva (2000).
} 
the population's real incomes. An empirical analysis of the interregional differentiation was conducted on the basis of the regression analysis of panel data. Basin on her assessments, the author concludes that the interregional differentiation of the population's incomes and the average GRP per capita intensified over the period in question. In addition, the author attempted to conduct a quantitative assessment of the impact of a number of factors (initial conditions of development, production structure, orientation towards exports, inflation rates, investment, and regional budget expenditures) on the dynamics of interregional biases. The respective outputs allow the author to argue that it is current economic indices (inflation, export, changes in the share of the services sector and the agrarian sectors) that provide a major contribution to the interregional differences, while possibilities for economic policy to influence the differentiation of regions' socio-economic positions are fairly limited.

The above review of research outputs presented in the national economic literature allows the following conclusions on the extent to which the issue of regional growth is examined therein:

1. Most papers essentially contain legal provisions regarding regional development policy options basin on the authors' personal views. Their empirical evaluations is mostly based upon a description of initial statistical data and parameters of dispersion of evaluated indices. In many cases, the authors fail to directly test, by using the available data, their economic policy conclusions and recommendations.

2. A number of papers describe the regions' development record and the dynamics of interregional differences, or they specify and correct the initial Goskomstat data. However, they fail to consider causes for such differences and factors underlying the dynamics of the respective indices, or they refer to them pro- 
ceeding from general economic considerations, without a quantitative anaylis.

3. While N. Mikheeev's paper appears most close to ours by its objectives and the degree of the empirical development of the issue, nonetheless, the period it covers (1990-96) falls under the first stage of reforms (the transformational slump). By contrast, at present we are keen to examine the regions' development at the stage of transition to growth and an initial stage of recovery growth. 


\section{The Concept of Convergence and Its Application to the Evaluation of Economic Growth in the RF Regions}

\subsection{The Convergence Theory}

The neoclassical model of economic growth bears an important specific feature: that is, it predicts the existence of conditional convergence, or the concept that forecasts a positive correlation between growth rates of a given economy and the difference between the current and equilibrium income level in the economy (steady state). The concept of conditional convergence appears different from the concept absolute convergence concept. The latter implies that poorer economies grow at a pace greater than richer ones (i.e., "catching up with" them). It may become possible that two economies meet the condition of conditional convergence (the economy's growth rates fall under the diminishing bias of the income level from its equilibrium state), but they fail to meet conditions of absolute convergence (the richer economies may grow at a higher pace than poorer, if the former find themselves farther from the steady state). The concepts would be identical only provided the steady state is identical fro both of them).

It rather frequently happens that the convergence hypothesis of the neoclassical growth rate is tested against the example of regions of the same country. Despite the regions may differ by the level of technological development, preferences, economic institutions, etc., the differences would be substantially less great than those between the countries. That is why the probability of the presence of an absolute convergence between the regions is substantially higher visa-vis that between different countries. However, the employment of regions for testing the absolute convergence hypothesis breaks an important prerequisite of the neoclassical model of growth: that is, 
the closeness of an economy. Obviously, cultural, linguistic, institutional and formal barriers to the moving of factors proves to be less significant for a group of regions of the same country. However, it was proved that even in the event the factors were mobile and, consequently, prerequisites of the original model were broken, a closed economy and the one with a free capital movement would bear similar dynamic features ${ }^{13}$.

The theory suggests that the initial differentiation forms an effect form exogenous shocks and an imperfect adjustment mechanism. In accordance with the convergence hypothesis, in the event at the initial moment the economy of a given country (region) is farther from the steady equilibrium state, its growth rates would be greater than those in the economy that finds itself closer to the equilibrium. Accordingly, in the longer run the differentiation should disappear. The convergence hypothesis is mostly frequently used for examining differences in, and the dynamics of the level of per capita GDP (GRP).

It should be noted, however, that there is no sole definition of the notion of "convergence" in the economic literature. Rather, research papers refer to several concepts of the hypothesis, with two ones being most popular: these are the so-called $\beta$ - and $\sigma$-convergences ${ }^{14}$.

While the concept of $\beta$ - convergence ${ }^{15}$ define convergence as a "catch-up" process, wherein poor countries or regions enjoy higher economic growth rates, the concept of $\sigma$-convergence ${ }^{16}$ is under-

\footnotetext{
${ }^{13}$ Barro, Sala-i-Martin (2004).

${ }^{14}$ For a more detailed review of convergence hypotheses and their empirical testing see: Barro, Sala-i-Martin (2004) and Le Pen (1998).

${ }^{15}$ The term $\beta$-convergence originates from the letter that denotes the coefficient under the initial per capita GDP in an assessed equilibrium. The term was introduced by Barro, Sala-i-Martin (1990).

${ }^{16}$ The term « $\sigma$-convergence» was introduced by Sala-i-Martin (1998).
} 
stood as a diminishment over time of the dispersion of distribution of the per capita GDP or any other income index applied to a sample of countries or regions.

The hypotheses of $\beta$-convergence and $\sigma$-convergence appear interrelated, albeit not equivalent. A number of papers ${ }^{17}$ demonstrate that $\sigma$-convergence does not directly follow from $\beta$-convergence. Henin, Le Pen (1995) suggested an interpretation of a correlation between an absolute $\beta$-convergence and $\sigma$-convergence. The former convergence points to the existence of a trend to reduction in the gap in the GDP per capita, while random shocks that affect countries' (regions') economies can counteract this particular trend and temporarily increase the dispersion of the distribution of GDP per capita.

To analyze the correlation between these tow kinds of convergence, let us consider a fundamental equilibrium of the neoclassical model of growth that correlate the growth rate in the per capita income in economy $i$ over some time interval to the initial income level:

$$
\log \left(\frac{y_{i, t}}{y_{i, t-1}}\right)=a_{i t}-\left(1-e^{-\beta}\right) \log \left(y_{i, t-1}\right)+u_{i, t}
$$

The theory shows that absolute term $a_{i t}$ is a sum of some variable that reflects the technological progress and the value whose multiplier is the logarithm of the equilibrium value of a given country's or region's income. This is the core of the concept of conditional convergence, as in this particular case one considers the income value that corresponds to a steady state of equilibrium.

While considering regions, it is assumed that absolute term $a_{i t}$ is the same for all of them. Given this, if $\beta>0$, then equation (2.1)

${ }^{17}$ For instance, Barro, Sala-i-Martin (2004). 
implies that poorer regions would enjoy high rates of economic growth. If on assumes that random $u_{i, t}$ has average zero, dispersion $\sigma_{u, t}^{2}$ and it dispersed independently of $\log \left(y_{i, t-1}\right)$ and $u_{j, t}$ for $i \neq j$, he can arrive to the following expression that allows to track down the correlation between $\beta$-и $\sigma$-convergences:

$$
\sigma_{t}^{2}=\frac{\sigma_{u}^{2}}{1-e^{-2 \beta}}+\left(\sigma_{0}^{2}-\frac{\sigma_{u}^{2}}{1-e^{-2 \beta}}\right) e^{-2 \beta t}
$$

where $\sigma_{0}^{2}-$ dispersion $\log \left(y_{i, 0}\right)$. It follows from this that $\sigma_{t}^{2}$ aims at its equilibrium value $\sigma_{u}^{2} / 1-e^{-2 \beta}$, which grows in parallel with the increase in $\sigma_{u}^{2}$, but diminishes with the increase in $\beta$. Over time $\sigma_{t}^{2}$ may grow or fall, depending on whether $\sigma_{0}^{2}$ is greater or smaller vs. an equilibrium value. So a positive value of coefficient $\beta$ still does not mean a diminishment of $\sigma_{t}^{2}$, i. e. the presence of the convergence. But $\beta$-convergence forms a necessary, but not sufficient conditions of the existence of $\sigma$ convergence. So, $\sigma$-convergence is noted only in the cases when $\beta$-convergence oppresses the impact of such random shocks. Let us also note that Lichtenberg (1994) extended this conclusion onto the conditional $\beta$-convergence as well.

The methodology of empirical testing concepts of convergence requires somewhat more detailed consideration. The most frequently used statistical method to test $\beta$-convergence is the regression of GDP growth rate (the average or accumulated over a given period) to the constant and logarithm of an original per capita GDP 
(on the basis of the cross-section data). Should the coefficient under the explanatory variable be statistically significant and get the negative sign, the hypothesis of absolute $\beta$-convergence is not rejected. However, there exist a number of problems that under an econometric assessment would lead to a biased assessment of coefficient $\beta$. More specifically, dispersion $\log \left(y_{i, t}\right)$ would appear vulnerable to perturbations that exert a general influence on a group of countries of regions. This would entail an abuse of the prerequisite of shocks $u_{i, t}$ being independent of different countries. In this particular case, such shocks would have a positive, or, on the contrary, negative impact on the countries or regions with a higher or lower income level, which is why the assessment of coefficient $\beta$ would be biased under the regression assessment. To solve this problem, one introduces to the equation additional variables that characterize the impact of these or those shocks. Providing the variable of the starting income level of a given country or region and additional variables, the econometric assessment of coefficient $\beta$ would be valid.

It is also important to note another major advantage of employing regional data to test the convergence hypothesis. As noted above, it mostly frequently happened that an empirical testing of convergence implied particularly assessment of the equation of a correlation between GDP growth rates and its initial level. However, according to the theory, the genuine correlation also comprise a term that considers the income value under the steady state. In other words, once the traditional approach to the assessment is employed, the regression equilibrium appears incorrectly specified. If coefficient $\beta$ has negative sign, the hypothesis of absolute convergence is not rejected. But the problem is if one needs to reject the convergence hypothesis, if he has got a positive assessment of co- 
efficient $\beta$, for the theory suggests a multiple correlation with the inclusion of a steady state variable.

If countries of regions converge to different states of the steady equilibrium, a regular pair regression appears incorrectly specified, and the member of an equation that reflects an equilibrium income value is included in the regression error. If it in turn is correlated with the variable of the country's or region's initial income level, the assessment of the coefficient of convergence $\beta$ would appear biased. For instance, should rich countries have a greater value of the equilibrium income, the assessment of the convergence coefficient would shift towards zero, which would entail incorrect conclusions on the absence of convergence, despite the existence of the conditional convergence. This example demonstrates the necessity of inclusion in a pair regression equilibrium of a proxy for the income in the steady equilibrium state to obtain a valid assessment of convergence coefficient $\beta$. In the event of an error in the regression equilibrium and the initial income level, the assessment of the pair correlation would allow to obtain a valid assessment of $\beta$. Finally, should all the countries or regions concerned enjoy an equal steady equilibrium state, the term that comprises an equilibrium income level would consequently form a part of the constant and the assessment of $\beta$ appears valid as well.

Hence, there exist two possibilities to obtain a valid assessment of coefficient $\beta$ : the first one is to find a proxy for the equilibrium income level and employ it as an additional explanatory variable, to assess a pair correlation between a given economy's growth rate and the income level at the initial moment of time. The other method is to use the data across economies that for sure have an equal equilibrium income level, or, at least, for whom the equilibrium and initial income levels are not correlated. It is the second method in the frame of which regional data appears particularly important. 
The contemporary literature has long attempted to assess the convergence hypothesis in respect to various countries or regions of a single one. Thus, Barro and Sala-i-Martin ${ }^{18}$ provide results of an assessment of $\beta$ convergence for the USA over the period 1880-2000 for the following equilibrium:

$$
(1 / T) \log \left(y_{i, T} / y_{i, 0}\right)=a+\left[\left(1-e^{-\beta T}\right) / T\right] \log \left(y_{i, 0}\right)+\omega_{i 0, T}
$$

Assessments along the whole period and its single sub-periods evidenced the presence of a convergence between different states. The assessment of coefficients $\beta$ along several subperiods bore the negative sign, but upon introduction of control variables that reflected a given state' geographical location and structural shocks, all the coefficients proved to be positive and statistically significant. Overall, it was found out that the convergence pace between different states accounted roughly for $2 \%$ annually.

Analogous assessments were carried out basing on the data on Japanese prefectures over the period between 1930 through 1990 . The respective results also testified to the presence of $\beta$ convergence: coefficient $\beta$ accounted for circa 0.028. Assessments of coefficient $\beta$ for single subperiods that had a wrong sign proved to be positive after inclusion of structural variables. Barro and Sala-iMartin also conducted assessments of convergence basing on the data on 90 regions in 8 European countries over the period between 1950 through 1990 (11 in Germany and UK, 20 in Italy, 21 in France, 4 in Holland, 3 in Belgium, 3 in Denmark and 17 in Spain). In contrast to the US and Japan, to account inter-country differences, the original convergence equation was supplemented by country-specific logical variables. The result was $\beta$ convergence

${ }^{18}$ Barro, Sala-i-Martin (2004). 
for the sample of regions in question, whose coefficient made up a. 0.02 . At the same time, the researchers were assessing a system of simultaneous equations to find coefficients $\beta$ for the top five largest countries: Germany, UK, Italy, France and Spain. In that case coefficient $\beta$ could vary across different countries, but not over time. The respective values ranged from 0.012 (France) to 0.027 (UK).

Under empirical testing of the concept of $\beta$ convergence the focus of attention is on the dynamics of the index of dispersion (the mean-square bias) of distribution of per capita GDP logarithms. Accordingly, if for a give sample of countries (regions) the dispersion of distribution of per capita GDP logarithms diminishes from the start through the end of the period in question, the hypothesis of $\beta$ convergence is not rejected. Such a method of testing this particular hypothesis appears purely descriptive. Thus, particularly Lichtenberg (1994) has modified the Fischer's test for a statistical testing if the diminishment of dispersion between the start through the end of the period was statistically significant.

While testing the hypothesis of $\beta$ convergence for the noted countries (the US, Japan and the European countries), the researchers examined if $\sigma$-convergence was present there. In particular, as already noted above, the researchers considered the dynamics of a standard bias of the per capita GDP (GRP) over the respective time periods. As long as the period between $1880-2000$ is concerned, the US had been experiencing the decline in the value of the standard per capita GDP. The exception was the period between 1920 through 1930, when this index was growing. That was likely to become an effect of a decrease of relative prices for agrarian products and thus affected the US agrarian states whose incomes had been relatively lower than elsewhere nationwide.

As concerns the Japanese prefectures, between 1930 through 1940 the GDP dispersion index had been growing there, but it con- 
sequently began decreasing up till the end of the period in question (1990). The analysis of dispersion of GDP of the European countries also evidenced the presence of a tendency to diminishment of the income level dispersion across the countries between 1950 through 1990. Thus, results of the analysis of the presence of $\beta$ convergence and $\sigma$-convergence for the noted groups of countries or regions completely correspond to each other.

In addition, to test the accuracy of the hypothesis of $\sigma$ convergence, one can also employ other indices that testify to a change in the inequality level between the countries or regions in terms of per capita income. Specifically, along with dispersion or standard bias indices, some papers employ the variation coefficient, which is square root of dispersion to the average value across the sample ratio. By contrast to dispersion or standard bias, once computed in such a way, the index will no longer depend on the unit of the examined revenue index. Furthermore, one may use a weighted coefficient of variation which is computed in a standard fashion, but considers weighted indices of average and the dispersion.

Another, rather popular, indicator of the inequality level is Gini coefficient computed on the basis of the Lorenz curve. To built the Lorenz curve, on the absciss axis one lays the accumulated proportion of the population of a group of countries or regions, while on the ordinate axis - the accumulated proportion of the countries' incomes in the total income across a given sample. While building the curve, one ranks all the countries concerned in the increasing order of the per capita income level. Once the Lorenz curve is built, one can compute Gini coefficient, which is a doubled difference between the area under the curve and a straight laid at angle of $45^{\circ}$. The analytical expression for the computation of Gini coefficient takes the following form: 


$$
G=\frac{1}{2 \bar{Y}} \sum_{i=1}^{N} \sum_{j=1}^{N} p_{i} p_{j}\left|Y_{i}-Y_{j}\right|
$$

where $p_{i}$ and $p_{j}$ - proportions of the population of countries $i$ and $j$ in the total number of population, $Y_{k}$ - index of income of country $k$. Accordingly Gini index can vary from 0 (complete equality) up to 1 (complete inequality).

To analyze the level of inequality between countries or regions in terms of per capita income, i.e. to test the concept of $\sigma$ convergence, one can also employ Tale index. It is borrowed from the theory of information and originally is associated with the concept of entropy. Tale index can be computed using the following formula:

$$
T(1)=\frac{1}{N} \sum_{i=1}^{N}\left(\frac{Y_{i}}{\bar{Y}}\right) \log \left(\frac{Y_{i}}{\bar{Y}}\right)
$$

One of the features of this particular index is the possibility for decomposition into two items, of which the first mirrors an inequality within each of the singled out groups of countries, while the other - an inequality within these groups. In addition, upon the division of the right part of the inequality into $\log (\mathrm{N}))$, the index value can range from zero to one.

It would also be worthwhile to focus on such a concept in the frame of the convergence theory as polarization. Despite it shows some similarity to the inequality concept, there is a considerable difference between them, nonetheless. According to Esteban and Ray $^{19}$, the polarization concept arise because of "inequality measurement axioms do not allow an adequate distinguishing of the convergence to the global median value from a clusterization

${ }^{19}$ Esteban, Ray (1994). 
around regular ones”. So, the polarization concept framework allows consideration of a possibility for emergence of clusters around local steady equilibrium states. The polarization concept can be illustrated using the following example: let us assume that there exists an originally noted even distribution of countries in terms of the level of GDP along the segment from 1 to 6 . Let us then consider the following transformation of the original distribution: the countries with income level from 1 to 3 converge to the state with income 2, while those with income level between 4 to 6 - to the state with income 5. Despite Tale index showing a reduction of the inequality, polarization grows. To compute the polarization index, the following formula is used:

$$
P I=\sum_{i=1}^{N} \sum_{j=1}^{N} p_{i}^{\alpha} p_{j}\left|Y_{i}-Y_{j}\right|
$$

where $\alpha$ - index from 1 to 1.6 that measures sensitivity of polarization. So, the smaller is the sensitivity index, the closer the polarization concept to the inequality concept is.

The consideration of convergence concepts also necessitates singling out a theory of convergence clubs. Convergence club is a group of countries (regions) for which the convergence conditions are met. Accordingly, there may exist a great number of convergence clubs with no convergence between them, which is correct in respect to any originally examined sample of countries or regions.

The emergence of convergence clubs is largely explained by the role of initial conditions. From the perspective of endogenous growth, to trigger a process of convergence between countries (regions), it is necessary for them to possess both an identical structure of their economies and similarity of their starting conditions. Galor (1995) showed that under certain prerequisites heterogeneity in the dynamics of savings can likewise lead to emergence of convergence clubs in the frame of the neoclassical theory of growth. 
Justification of a possible composition of such clubs pose a fundamental complex problem under empirical testing of this particular convergence concept. There are two most frequently used methods to do this. According to the first method, the composition of clubs is originally determined on the basis of criteria the researcher selects, while the other method implies "endogenization" of the selection of clubs, under which the researcher has to find any statistical method that would allow to single out convergence clubs in the given data series. Once the composition of clubs is identified, the researcher would just need to examine if any of the existing convergence criteria is met within each singled out group of countries (regions).

In addition to the aforementioned methods of testing convergence concepts, there exist other methods of their analysis. For instance, it was suggested to employ methods of the tome series theory to test the convergence hypothesis econometrically: specifically, Bernard (1991), Quah (1992), Bernard, Darlauf (1998) have introduced the concept of stochastic convergence. The hypothesis of stochastic convergence is met if for two given countries the difference between their per capita income levels is a stationary process with zero average. In such particular case it can be argued that the economies in question have reached their own state o steady equilibrium and shocks that affect them are short-term.

Evans (1996), in turn, considered statistical characteristics of a time series of the per capita GDP logarithms, which was built upon indices of dispersion at each given moment of time along the whole given time interval. If, as the neoclassical theory of grows forecasts this, long-term paths of GDP per capita in given countries (regions) are parallel, their dispersion series should appear stationary relative to a positive constant. If, as the theory of endogenous growth argues, GDP=s per capita grow at different paces, the series 
of dispersions should be integrated of the first order with an immanent square trend.

There exist, at least, three interrelated concepts of stochastic convergence. The concept of strict convergence (asymptotically perfect convergence) is true only in the event the difference between two tome series of any given countries from a given sample does not contain either unit root, or trend (no matter determinist or stochastic). In other words, it should meet the following condition:

$$
\lim _{T \rightarrow \infty} E\left(Y_{i, T}-Y_{j, T} \mid \xi_{0}\right)=0,
$$

where $\xi_{0}$ - all the existing information as of the time period T. However, this concept of stochastic convergence is often criticized for its rather strict conditions: it suggest that a long-term envisaged value of the difference between per capita income levels in two countries is zero. That is why researchers consider an alternative concept of a weak stochastic convergence (asymptotically relative convergence), according to which the noted difference between time series of two countries converge not to zero, but to some constant. This can be expressed formally in the following way:

$$
E\left(Y_{i, T}-Y_{j, T} \mid \xi_{t}\right)<\left(Y_{i, 0}-Y_{j, 0}\right),
$$

where 0 corresponds to the current moment of time, while $\mathrm{T}-$ to some moment of time in the future. According to noted definition, the difference between two time series should also be stationery, but allows the presence of a determinist time trend.

Finally, a less rigorous concept of stochastic convergence is correct in the event when, despite the existence of different trends for two time series, there exists their linear combination that satisfy the following condition: 
$\lim _{T \rightarrow \infty} E\left(Y_{i, T}-\beta Y_{j, T} \mid \xi_{0}\right)=0, \quad \beta>0$.

Hence, the approach based on the time series theory suggests that series of per capita GDP logarithms can comprise both stochastic and determined trends. If so, then the analysis of time series should aim at studying into a correlation between determined and stochastic trends that determine the dynamics of per capita GDP. The hypothesis of stationarity means that time series have both determined and stochastic trends, i.e. they appear co-integrated (a cointegral correlation allows constant, but not a linear trend), and their dynamics is determined by identical factors.

In other words, convergence is understood as the preservation over time at the level close to zero of the existing gap between two countries in the per capita GDP levels. Evidently this condition is to a greatest extent applicable to groups of countries with close per capita GDP levels, albeit it fails to explain differences between "rich" and "poor" countries' living standards. It should be noted that the concept of convergence that assumes the gap between levels of per capita GDP $=$ s contradicts the condition of $\beta$ convergence. Bernard, Durlauf (1998) explained this contradiction arguing that a convergence with a constant gap falls under the case of economies moving along steady long-term paths of growth, while $\beta$ convergence describes a period of transition from one path to another.

While the concept of stochastic convergence allows to lift a number of problems that arise in the course of consideration of $\beta$ - and $\sigma$-convergence, it suffers a number of drawbacks. Thus, particularly, the existing tests of stationarity (the extended DickyFuller test or the Phillips-Perron test) enjoy a low statistical capacity, as far as final samples are concerned. With such a capacity, the probability of a non-rejection of zero hypothesis of the existence of the unit root is high. 
Another concept of convergence considers an evolution of a relative position of each country vis-à-vis the others. The author of the concept was Quah $^{20}$ who believed that the concepts of $\beta$-convergence and unconditional and conditional $\beta$-convergences have nothing to do with the notion of convergence per se. That is why he suggested studying the process of convergence basing on an evaluation of the dynamics of the overall distribution of the multitude of per capita GDP values of a give sample of countries. Quah does not reject a hypothesis of convergence, if the distribution of per capita GDP for the given group of countries or regions tends in time to a unimodal one, while in the event of a bimodal distribution, the polarization concept becomes correct. Under the polarization concept the group of countries with an average income level disappears. As well, Quah stresses the necessity of assessing the scale of a change of a relative position a given country holds within the overall distribution.

Hence, whilst proceeding to the empirical analysis of convergence processes between Russia's regions, it becomes possible to conclude that at present there exist a few convergence concepts in respect to different countries or regions of a given country, which consequently implies a few methodologies of the analysis of convergence. First, the simplest way to analyze the presence of a convergence in the economic development rates implies the perspective of reduction in an inequality in terms of per capita GDP (GRP) between a group of regions (i.e. to consider the $\sigma$-convergence concept). This particular approach implies most frequently an analysis of various indicators that characterize the level of inequity of the given countries or regions in terms of some index of income, most frequently - GDP (GRP). Secondly, to test the $\beta$ - convergence concept, researchers most frequently employ

${ }^{20}$ Quah (1993, 1995, 1996). 
cross-section analysis across a given sample of countries or regions. Thirdly, testing of convergence concepts on the basis of the analysis of time series of income indices and studying their dynamic characteristics appears somewhat more sophisticated from the methodological perspective. However, in our case the absence of relatively long series of data across Russia's regions does not allow to employ the time series method to test a convergence hypothesis.

\subsection{An Empirical Testing of Convergence Concepts in Respect to the RF Regions}

2.2.1. Comparative Analysis of the RF Regions

by GRP over the Period between 1994 through 2002

For the purpose of statistical analysis we used statistical data of the RF Goskomstat across 88 regions $^{21}$ of the Russian Federation over the period between 1994 through 2002. Before an empirical testing of convergence concepts in respect to Russia's regions, we have conducted a comparative analysis of regions by the size of their regional income. For this purpose, we employed per capita GRP in the 1994 prices. The adjustment of GRP per capita values to the 1994 prices was made by means of regional CPI=s. Bar charts of dispersion of regions by GRP per capita values between 1994 to 2002 are given in Fig. 2.1, while main statistical characteristics for each years are presented in Table 2.1.

\footnotetext{
${ }^{21}$ Because of the absence of the data on a number of its macroeconomic indicators, Chechen Republic was excluded from the group of examined regions.
} 

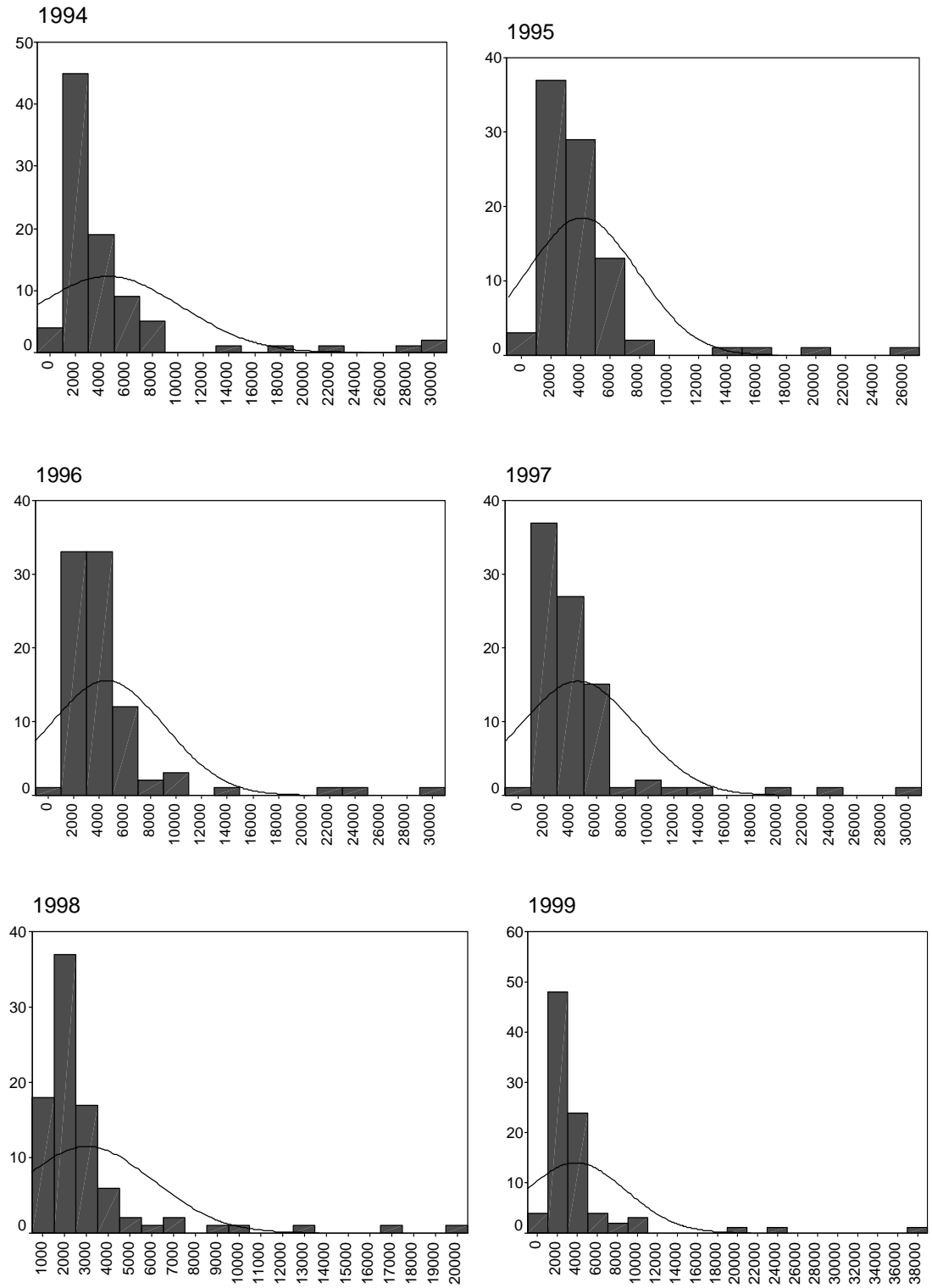

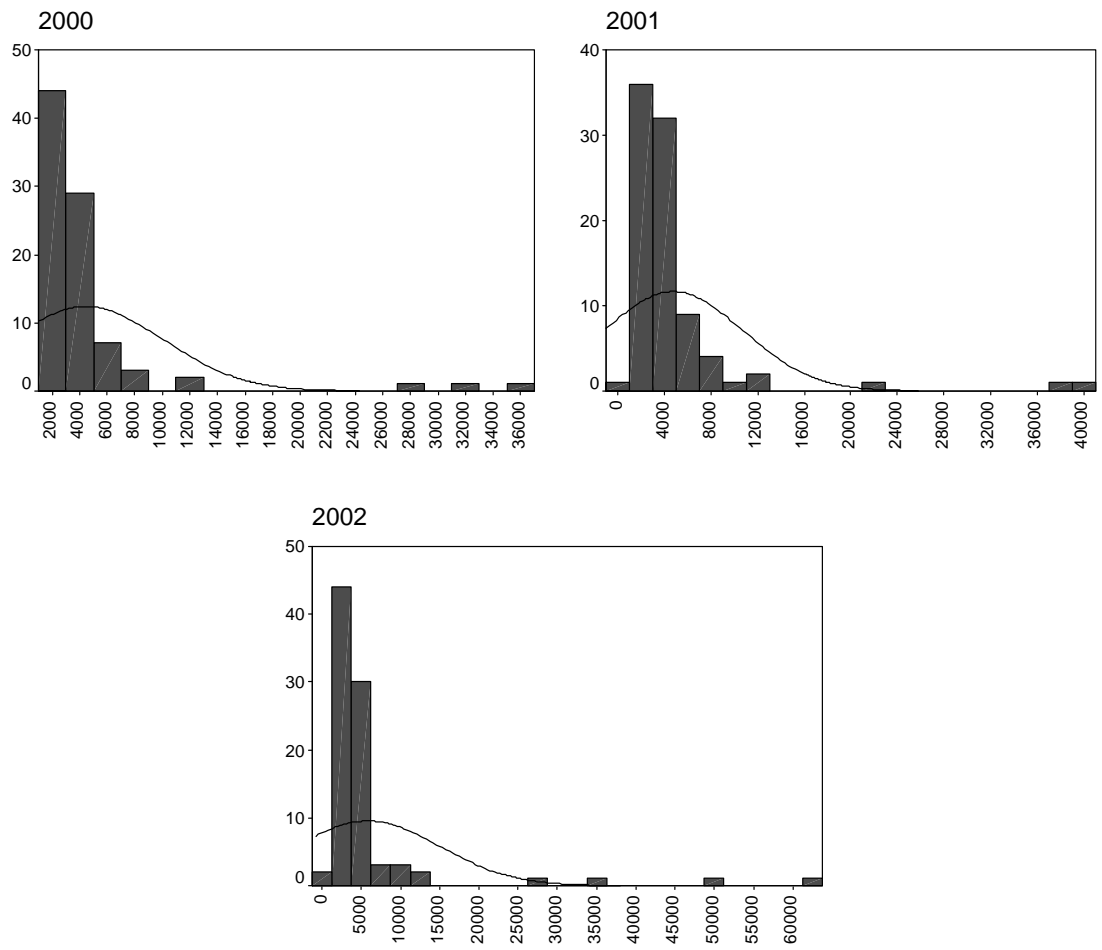

Fig 2.1. Dispersion of Russia’s Regions by level of GRP Per Capita in Constant Prices (as Rb. Th.) in 1994-2002

Table 2.1

Main Statistical Characteristics of GRP Series between 1994 to 2002

\begin{tabular}{lccccc}
\hline & Minimum value & Maximum value & Average value & Median & Standard Bias \\
\hline 1994 & 458.12 & 30802.78 & 4538.58 & 2867.05 & 5694.55 \\
1995 & 449.53 & 26318.35 & 4124.38 & 3119.03 & 3791.86 \\
1996 & 414.31 & 30096.50 & 4567.54 & 3293.81 & 4508.34 \\
1997 & 414.33 & 30339.85 & 4610.18 & 3281.92 & 4534.38 \\
1998 & 734.23 & 19809.48 & 3018.30 & 2159.68 & 3050.02 \\
1999 & 641.83 & 37185.51 & 3820.46 & 2440.29 & 5000.13 \\
2000 & 1133.73 & 36972.71 & 4459.18 & 3007.17 & 5666.30 \\
2001 & 948.21 & 39073.32 & 4764.78 & 3279.82 & 5997.33 \\
2002 & 622.81 & 63690.17 & 5831.56 & 3590.75 & 9171.42 \\
\hline
\end{tabular}


The above bar charts allows to note that throughout the whole period in question, roughly as many as half of the regions had their GRP per capita at the level not more than Rb. 2,000, while some two-thirds of regions - at the level of not more than 4,000. Given that, the dispersion of regions in terms of GRP per capita level for each of the years in question appears unimodal, which, following Quah (see above) testifies to the presence of convergence processes.

Overall, during the whole period in question the average GRP value was at the level $\mathrm{Rb}$. 4,500 per capita, with a notable fall in this index down to 3,000 in 1998, in the wake of the 1998 crisis. However, since 1999 the regional per capita incomes have been growing and by 2002 they practically doubled vis-à-vis the minimum value and reached Rb. 5,800. Notably, as far as the time period starting from 1999 is concerned, the rise in the average GRP value per capita was also accompanied by a steady rise in the median GRP per capita value. In other words, the rise in the average living standards was generated both by the further increase in the GRP per capita level in the most prosperous (capital cities and oil producing regions) and by improving living standards in poor regions. It should be noted that there was no such picture in 19941997 and, accordingly, positive changes in average indices could be triggered by marginal values.

Given the above, a gradual rise in GRP per capita over several recent years has been also characterized by a broadening gap between regions, which is evidenced by both a steady excess of the average per capita GRP value over the median one and by a growing index of standard bias of GRP in 2002. One can single out a number of regions for which the GRP per capita value appears substantially greater vs. the average one. This is true for the period between 1994 through 2002 for such regions as Nenetsky AO, Khanty-Mansi AO, Yamalo-Nenetsky AO, Altay Republic, Repub- 
lic of Khakassia, Republic of Sakha (Yakutia), Tyumen oblast, and the city of Moscow.

\subsubsection{Testing Hypothesis of $\sigma$-Convergence}

The concept of $\sigma$-convergence is accurate in the event there exists a decrease in the dispersion of the GRP per capita index for a group of regions. In other words, if $\sigma_{t+T}<\sigma_{t}$, where $\sigma_{t}$ is dispersion index, there exists $\sigma$-convergence. To test this hypothesis, researchers most often employ the index of dispersion, standard bias or variation coefficient. Despite the fact nowadays they most often apply the two firs indices (dispersion or standard bias), these particular indices suffer a drawback, which manifests itself in their dependence on the measurement unit of a given economic variable. Because of this, to describe an disparity between regions, one would employ the variation coefficient that is computed in the following way:

$$
C V=\frac{\sqrt{V}}{Y_{\text {avg }}}
$$

where $V=\frac{1}{N} \sum_{i=1}^{N}\left(Y_{i}-Y_{a v g}\right)^{2}$ - dispersion of GRP per capita, $Y_{\text {avg }}-$ average GRP per capita value for regions, $N$ - the overall number of regions.

By its structure, the index $C V$ does not take into account a relative number of regional population, which can be considered by means of the weighted variation coefficient $-C V_{W}$, which is computed by analogue with (2.10), except for the dispersion index, which is modified for the purpose of taking into account the weight of the share of the population of a single region in the total number 
of population $\left(p_{i}\right)$ using the following - hereinafter referred to as «Weighted Correlation Rate (method 1)"22:

$$
V_{W}=\frac{1}{N} \sum_{i=1}^{N}\left(Y_{i}-Y_{\text {avg }}\right)^{2}\left(1-p_{i}\right) .
$$

There also exists another way to compute the weighted dispersion index that takes into account an average weighted value. It can be computed as follows (hereinafter referred to as Weighted Correlation Rate (method 2)":

$$
V_{w}=\sum_{i=1}^{N}\left(Y_{i}-Y_{a v g}\right)^{2} p_{i} .
$$

Yet another, rather popular, index that characterize the disparity level between countries (regions) in terms of income level is Gini coefficient, which is computed by Lorenz curve. The latter is a correlation between the accumulated share of the regions' GRP=s per capita in the total GRP and the accumulated share of the regions' population in the overall number of population. To build this particular curve, all the regions are ranged by the increase of the GRP per capita index.

To analyze the disparity level between countries or regions in terms of the GRP index, along with coefficient Gini, economists also employ Tale index computed according to the following formula:

$$
T(1)=\frac{1}{N} \sum_{i=1}^{N}\left(\frac{Y_{i}}{\bar{Y}}\right) \log \left(\frac{Y_{i}}{\bar{Y}}\right) .
$$

${ }^{22}$ See: Castro (2004). 
By analogy with Gini coefficient, should Tale index equal zero, it would mean a complete equality in terms of the index concerned, while once it equals $1,-$ a complete disparity.

Values of the noted characteristics of disparity level computed for the index of GRP per capita level in constant 1994 prices are given in Fig. 2.2 and 2.3. Fig. 2.2 illustrates that throughout the period in question there has been no decline in the variation coefficient, while it has begun to grow roughly since 1998. In other words, since that moment the disparity between Russia's regions in terms of the value of their GRP=s per capita has begun growing.

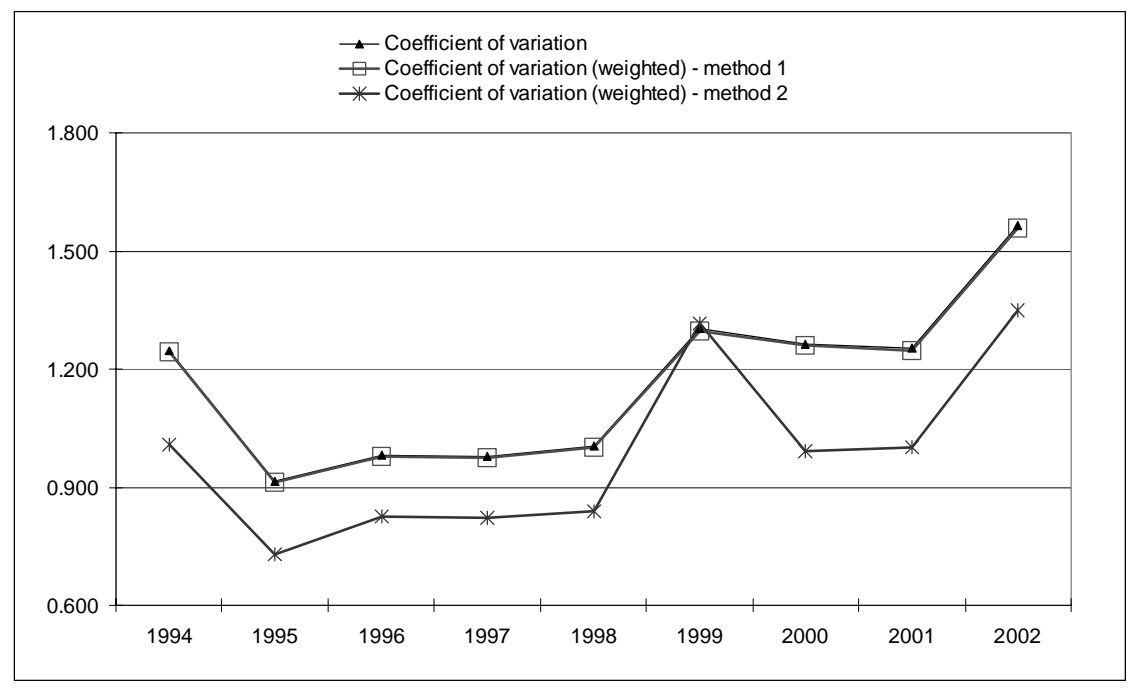

Fig 2.2. Simple and Weighted Variation Coefficients of the Index of GRP Per Capita for Russia's Regions 


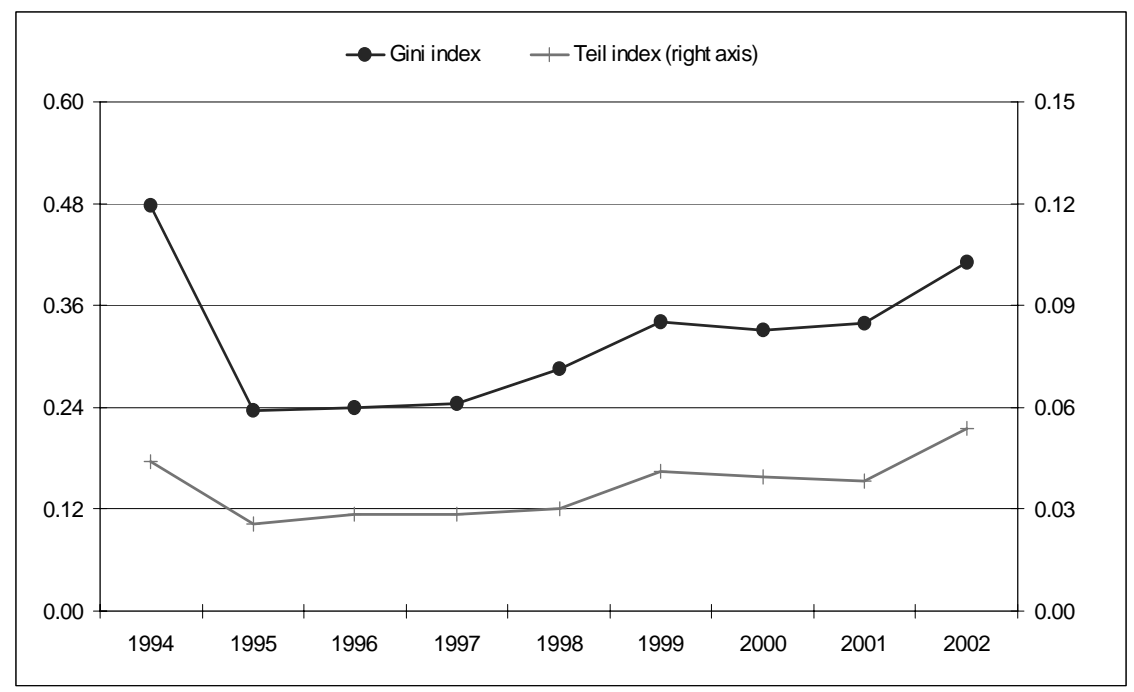

Fig. 2.3. Dynamics of Gini and Teil Indices for Russia;s Regions Computed for GRP Per Capita Index

The absence of a diminishment in interregional disparity in terms of GRP per capita level follows from the dynamics of Gini and Teil indices presented in Fig. 2.3. While in the beginning of the period in question there occurred a drastic single drop of disparity between regions in terms of GRP per capita (in 1995), the disparity level has been remaining practically stable for a few years afterwards. Furthermore, since 1998 onwards one noted the predominance of a trend to growth in the inter-regional disparity in terms of income level.

Summing up the research outputs, it can be argued that the concept of $\sigma$-convergence has failed to find a proof in the data on Russia's regions. Our results show that the level of inter-regional disparity by the value of per capita GRP (in constant prices) does not fall. Rather, it has been growing over past 4-5 years. The analysis of various indicators of disparity has exposed similar results. The 
comparison of the results with conclusions drawn on the basis of an analysis of characteristics of regional income indicators in Section 2.2.1 allows to conclude that regardless of the growth in disparity, an absolute level of per capita GRP was growing nonetheless. Such a growth was noted in respect to the sample on average and among "rich" and "poor" regions.

\subsubsection{Testing the Concept of the Unconventional $\beta$-convergence}

The existence of $\beta$-convergence implies a negative statistical correlation between the growth rate of income per capita and its initial level under a cross-sectional analysis of countries or regions. A specification of the regression model finds itself determined by the kind of $\beta$-convergence to be tested. Thus, of one assesses a paired regression correlation of the growth rate of income index by constant and an initial level of this indicator, it becomes possible to test the existence of an absolute convergence. But if a given equation comprise additional exogenous parameters that characterize a difference in the level of production technologies, savings rates, population growth rates, and a number of other parameters, it is the hypothesis of conditional convergence that is tested.

It is first of all necessary to analyze results of an assessment of the paired regression correlation between the value of the 2002 GRP per capita growth rate vs. 1994 (in annual terms) and the initial level of GRP in 1994, i.e. the existence of an absolute (unconditional) $\beta$-convergence. Results of the assessment of the correlation are given in Table 2.2.

The table demonstrates that the concept of $\beta$-convergence is applicable to Russia's regions over the whole period in question. This is evidenced by the negative and statistically significant coefficient under the variable of the1994 GRP logarithm. So, the regions with a smaller level of their 1994 GRPs enjoyed greater rates of growth 
in GRP between 1994 through 2002. The convergence rate at this junction accounts for some $0.825 \%$ annually, which is a moderate indicator for such computations (for reference: in Barrow and Salai-Martin's papers the respective rates laid within 2-3\% annually).

Table 2.2

Results of the Assessment of Correlation between GRP Per Capita Growth Rates and the Initial Level of GRP

\begin{tabular}{|c|c|c|}
\hline Explained variable & Logarithm of GRP & $\begin{array}{l}\text { ate in constant prices } \\
\text { ed) }\end{array}$ \\
\hline \multirow[t]{2}{*}{$\begin{array}{l}\text { Number of observa- } \\
\text { tions }\end{array}$} & \multicolumn{2}{|c|}{ e } \\
\hline & Coefficient & $P$-value $t$-стат. \\
\hline Intercept & 0.561 & 0.000 \\
\hline $\begin{array}{l}\text { Logarithm of the } 1994 \\
\text { GRP per capita }\end{array}$ & -0.066 & 0.000 \\
\hline Adj. $R^{2}$ & \multicolumn{2}{|c|}{0.298} \\
\hline$P$-value $F$-statistics & \multicolumn{2}{|c|}{0.000} \\
\hline
\end{tabular}

2.2.4. The Impact of Budget Policy on Convergence between Russia's Regions: Testing the Hypothesis of Conventional $\beta$-convergence

So, the results of our assessments do not reject the hypothesis of an absolute $\beta$-convergence for Russia's regions over the period between 1994 through 2002. More than that, the convergence rates have proved to be extremely high. That is why we are keen to examine the role played by economic policy and, particularly, interbudgetary transfers from the federal center and capital investment of regional budgets in reaching conditions of an interregional convergence. It should also be noted that since 2002 the RF Government has implemented the federal targeted program "Reduction in differences in socio-economic development of regions of the Russian Federation (for 2002-2010 and until 2015)”, however, whereas the official information on GRP over the period 2003-04 so far has 
been unavailable, we have no opportunity to test the hypothesis of efficiency of this program.

\section{Box 1}

The development of the federal targeted program "Reduction in differences in socio-economic development of regions of the Russian Federation (for 20022010 and until 2015)"was launched in March 2001. The program is based upon "The main guidelines of socio-economic policy of the Government of the Russian Federation over the long-term perspective" and "The action plan of the Government of the Russian Federation in the social policy area and the economy modernization over 2000-01". The public customer of the program is the RF Ministry of economic development and trade, while the principal designer of the program is the Council for studying into production forces under the RF Ministry of economic development and trade and the RF Academy of Sciences.

The Program objective is to diminish differences in the level of RF regions' socio-economic development; reduction in the gap by main indicators of socio-economic development between the most developed and backward regions by 1.5 times by 2010 and 2 times by 2015 .

The main goals of the Program are:

- Fostering conditions of development of the regions whose socioeconomic indicators are lower than average nationwide ones;

- Creation of a favorable environment for development of entrepreneurial operations and improvement of the investment climate;

- Increase in efficiency of the governmental support to the RF Subjects.

The Program implementation is scheduled for 2002-2015

The total volume of the Program funds is $\mathrm{Rb} .66323,3 \mathrm{mln}$, including:

- $\quad$ The federal budget funds Rb. $12413.3 \mathrm{mln}$;

- $\quad$ Budgets of RF Subjects - Rb 15168 mln;

- $\quad$ Extrabudgetary funds - Rb 38742 mln.

The envisaged Program final results are:

- Reduction in interregional differences between the RF Subjects in terms of the level of GRP per capita with account of purchasing power and per capita incomes by two times by 2010 and another $25 \%$ by 2015 ; 
- Contraction of the share of the population below the poverty line by $15 \%$ by 2010 and $25 \%$ by 2015 .

In 2002 alone, it was reported that works were complete on construction and reconstruction of social infrastructure facilities in backward in terms of socio-economic development regions. This would allow to bring the regions closer to the average nationwide development level. The year 2003 saw a continuation of the construction of water supply, heating and gas supply facilities. According to preliminary assessments, in 2004488 construction projects were completed in 45 Russian regions (243 social facilities, 83 educational, 81 - healthcare, 79 other social facilities). As well, 245 engineering facilities (water supply and gas supply) were build and repaired.

In 2002, from all the sources Rb. 3599.7 was earmarked (or $94.5 \%$ of the planned amount) to fund the noted works, of which:

- $\quad$ Federal budget funds $\mathrm{Rb}-1958,9 \mathrm{mln}$., or $100 \%$ of the limit of budget obligations;

- $\quad$ RF Subjects' budget funds - Rb.1238 mln., or 91,2 \%;

- Extrabudgetary funds- Rb. 402,8 mln., or 81,7 \%.

The following regions have failed to earmark the planned financial resources:

- From regional budgets: Kirov oblast (78,2\%), Republic of Khakassia (73,8\%), Republic of Mordovia (75,3\%), Moscow (71,1\%), Ivanovo (59,5\%), Orel (35,8\%), Kurgan (26,8\%), Omsk (4,1\%), oblasts, Tyva Republic $(35,7)$;

- From extrabudgetary sources: Altay Krai (23,9\%), Novosibirsk (67,6\%), Orel (33\%), Tomsk (18,6\%), Tula (33\%) oblasts.

The Program comprised R\&D implemented in 2002 for the purpose of developing a methodology of selection of regions and projects under the Program to be funded from the Fund for Regional Development.

In 2003, the Program allowed to finance 331 facility in 42 RF Subjects, of which 127 were social infrastructure facilities, and 204 engineering infrastructure facilities. Another 96 facilities were put into operation. It should be noted that de-facto only Rb.4295.6 was earmarked from all the sources, or $50 \%$ of the Program, with the federal budget alone honoring its obligations in full. In 2003, from the federal budget at the expense of the Fund for Regional Development Rb 2645.6 mln. was earmarked on the Program implementation, which accounts for $99.8 \%$ of annual limits. The RF Subjects' budgets ear- 
marked Rb.1257.1 mln.,or $73.4 \%$ of the respective limits(65,9\% of the Program Passport). The most alarming situation was noted in respect to the Program financing from extrabudgetary sources, with just as much as Rb 392,9 mln., or $65.4 \%$ of the envisaged limits ( $7.8 \%$ of the Program Passport) earmarked.

To test the impact the noted measures have on convergence processes, we assessed the regression model earlier employed for testing the hypothesis of an absolute convergence. The model was complemented by controlling variables that reflect the volume of the federal financial aid and investment in capital assets funded from the budget sources. That de-facto equals the testing of the hypothesis of a conditional $\beta$-convergence where these economic policy measures are recognized as convergence conditions.

Specifically, we used three controlling variables: the average value over the period (1994-2002), the volume of an aggregate financial aid from a superior-tier budget to GRP ratio, and the averaged over the period (1994-2002) investment in capital assets funded from the budgets of all levels to GRP ratio. The hypothesis of conditional convergence suggests that in such a regression the sign at the initial level of GRP per capita should be (as before) negative, while the one with the controlling variable - positive, i.e. a greater volume of transfers to a given region, or a greater volume of investment from the budget sources in it result in a more rapid growth of GRP per capita. Results of the assessment of the model with the noted controlling variables are given in Table 2.3.

As the above assessments show, in all the cases the coefficients at the controlling variables bear negative sign and a low statistical significance, while the assessments of the coefficient at the initial GRP per capita have remained statistically significant and close to the case of testing the hypothesis of unconventional convergence. The respective results can be interpreted as an evidence of the fact that the hypothesis of unconventional convergence, indeed, cannot 
b rejected for Russia's regions for the period 1994-2002, however, the role of the government regional economic policy in this phenomenon is extremely negligible. Thus. Negative signs at the controlling variables mean that the regions that received greater transfers from the federal budget were demonstrating relatively more modest GRP per capita growth rates (a low statistical significance of the assessments, however, does not allow arguing that this statement is proved).

Table 2.3

Results of Assessment of the Correlation between the GRP Per Capita Growth Rates and the Initial Level of GRP with the Inclusion of Additional Controlling Variables

\begin{tabular}{cccc}
\hline Explained variable & \multicolumn{2}{c}{$\begin{array}{c}\text { Logarithm of GRP per capita growth rate in constant prices } \\
\text { (1994-2202, annualized) }\end{array}$} \\
\hline Number of observations & $\begin{array}{c}\mathbf{8 8} \\
\text { Coefficient }\end{array}$ & $\begin{array}{c}\mathbf{8 8} \\
\text { Coefficient } \\
0.673^{* *}\end{array}$ & $\begin{array}{c}\mathbf{8 8} \\
\text { Coefficient } \\
0.672^{* *}\end{array}$ \\
$\begin{array}{c}\text { Constant } \\
\begin{array}{c}\text { Logarithm of GRP per capita } \\
\text { growth rate in 1994 }\end{array}\end{array}$ & $-0.066^{* *}$ & $-0.076^{* *}$ & $-0.077^{* *}$ \\
$\begin{array}{c}\text { Financial aid to the region to } \\
\text { GRP ratio }\end{array}$ & -0.043 & - & - \\
$\begin{array}{c}\text { Financial aid to the region to } \\
\text { the regional budget revenue } \\
\text { ratio }\end{array}$ & - & $-0.085^{*}$ & - \\
$\begin{array}{c}\text { Investment in capital assets } \\
\text { from budget sources to GRP } \\
\text { ratio }\end{array}$ & - & & $-0.692^{*}$ \\
Adj. $\mathbf{R}^{2}$ & & - & 0.350 \\
$\boldsymbol{P}$-value $\boldsymbol{F}$-statistics & 0.294 & 0.376 & 0.000 \\
\hline
\end{tabular}

Note: ${ }^{* *}$ - assessment is statistically significant at the $5 \%$ level, ${ }^{*}$ - assessment is statistically significant at the $10 \%$ level.

$$
* * *
$$

The results of the present empirical analysis of the concept of convergence between Russia's regions appear ambiguous.

First, the average characteristics of distribution of GRP per capita were growing during the whole period concerned (1994-2002), 
which evidences general living standards rise. The Inter-regional differentiation is growing too, nonetheless. However, since 1999 the rise in the average level has been noted in parallel with that of the median value of GRP per capita, which means that the rise in the level takes place both thanks to a further increase in the richest regions' welfare and the rising per capita incomes in poor regions. The distribution of regions in terms of per capita income remains unimodal.

Second, the hypothesis of $\sigma$-convergence was rejected by all the tests we employed.

Thirdly, results of the regression analysis evidence that the concept of unconventional $\beta$-convergence appeared just, as far as Russia's regions are concerned. In other words, for the whole period in question, the regions with a lower GRP indices in 1994 demonstrated greater growth rates of the index by 2002.

Fourthly, an additional analysis of the impact of the federal financial aid and the budget investment policy on GRP growth rates (the hypothesis of conditional $\beta$-convergence) showed the absence of a correlation of this kind. More than that, results of our assessments were likely to testify to a negative effect the regional economic policy (or to its use in pursuance of opposite objectives) on growth in regions.

In conclusion it is necessary to compare these results with those of one of the earlier CEPRA projects ${ }^{23}$, that focused on testing hypotheses on whether various fiscal instruments and, more particularly financial aid to Russian regions, bear a progressive or regressive feature. The 2003 paper argued that this or those fiscal instrument bears either feature in relation to any economic or financial indicator if the employment of the given financial instrument contributes to a lowering of the level of disparity the instrument meas-

${ }^{23}$ Kadochnikov, Sinelnikov at al (2003). 
ures. The authors of the cited paper acquired results that proved that the system of distribution of financial aid bore (only within single time intervals) the progressiveness feature relative to GRPs of Russia's regions. However, while assessing a stabilization effect of the federal fiscal system, the authors have failed to identify a stable significant negative correlation between the increment in the financial aid and the increment in GRP.

Hence, our results (in the part of the analysis of the impact of the regional budget policy on convergence processes) to a significant extent correspond to the conclusions drawn at the prior stages of CEPRA project: that is, financial aid to regions has failed to steer a more rapid economic growth in GRP per capita (i.e. it equally failed to accomplish its stabilization mission), or given other conditions being equal, the regions with a greater initial income level received a greater volume of transfers from the federal budget (the progressiveness is rejected during the whole period).

The absence of the effect on growth on the part of investment in capital assets financed out of the budget sources proves results of yet another project ${ }^{24}$ : that is, the efficiency of budget investment is extremely low, while they are focus chiefly on the socio-political area.

${ }^{24}$ Dneprovskaya, Drobyshevsky at al. (2002). 


\section{Annex 1}

Table A1-1

GDP Per Capita in 1994 Prices (as Rb. Th.) over 1994-1998

\begin{tabular}{|c|c|c|c|c|c|}
\hline & 1994 & 1995 & 1996 & 1997 & 1998 \\
\hline 1 & 2 & 3 & 4 & 5 & 6 \\
\hline $\begin{array}{l}\text { Aginsky Buryatsky } \\
\text { autonomous okrug }\end{array}$ & 1045.96 & 1153.68 & 1358.46 & 1443.38 & 886.79 \\
\hline Altay krai & 5035.78 & 2306.01 & 2724.11 & 2432.88 & 1518.17 \\
\hline Amur oblast & 4103.75 & 3646.13 & 4521.74 & 4997.82 & 2832.81 \\
\hline Arkhangel' oblast & 3497.61 & 3907.27 & 4292.99 & 4615.46 & 2968.36 \\
\hline Astarkhan oblast & 2104.30 & 2147.94 & 2454.95 & 2482.10 & 1744.86 \\
\hline Belgorod oblast & 2804.66 & 3725.07 & 3403.77 & 3380.54 & 2290.55 \\
\hline Bryansk oblast & 2242.09 & 2027.42 & 2472.33 & 2135.68 & 1300.76 \\
\hline Vladimir oblast & 2542.11 & 2704.99 & 2779.13 & 2880.12 & 1810.75 \\
\hline Volgograd oblast & 3187.38 & 3024.81 & 3684.51 & 3500.68 & 2098.81 \\
\hline Vologda oblast & 4550.07 & 5726.46 & 4699.52 & 4429.18 & 3309.89 \\
\hline Voronezh oblast & 2353.53 & 2751.43 & 2589.31 & 2677.88 & 1593.63 \\
\hline City of Moscow & 6203.83 & 6943.02 & 9498.83 & 11334.06 & 6374.41 \\
\hline Saint Petesrburg & 3506.72 & 4257.41 & 4820.14 & 4901.02 & 3382.35 \\
\hline $\begin{array}{l}\text { Jewish Autonomous } \\
\text { oblast }\end{array}$ & 2855.66 & 2358.93 & 2369.80 & 2368.13 & 1320.47 \\
\hline Ivanovo oblast & 2049.49 & 2120.43 & 2287.24 & 1971.52 & 1255.78 \\
\hline Irkutsk oblast & 1496.07 & 5113.48 & 5503.27 & 5939.78 & 3410.96 \\
\hline $\begin{array}{l}\text { Kabardino-Balkar } \\
\text { Republic }\end{array}$ & 1144.68 & 1279.02 & 1727.49 & 1664.17 & 1257.94 \\
\hline Kaliningrad oblast & 2482.40 & 2350.77 & 3045.47 & 3100.51 & 1630.48 \\
\hline Kaluga oblast & 2737.84 & 2848.47 & 2810.81 & 2748.23 & 1647.58 \\
\hline Kamchatka oblast & 5087.93 & 5489.86 & 6358.88 & 5801.24 & 5070.45 \\
\hline $\begin{array}{l}\text { Karachay-Cherkess } \\
\text { Republic }\end{array}$ & 1613.53 & 1696.95 & 2090.94 & 2049.89 & 1248.09 \\
\hline Kemerovo oblast & 2883.11 & 5162.84 & 5560.97 & 5004.32 & 2993.38 \\
\hline Kirov oblast & 2651.19 & 3127.45 & 3337.49 & 3333.71 & 2074.53 \\
\hline $\begin{array}{l}\text { Komi-Permyak } \\
\text { autonomous okrug }\end{array}$ & 2101.87 & 2579.00 & 2402.45 & 2440.81 & 1190.01 \\
\hline $\begin{array}{l}\text { Koryak autonomous } \\
\text { okrug }\end{array}$ & 8194.26 & 7836.62 & 9163.61 & 8491.25 & 9023.29 \\
\hline Kostroma oblast & 2814.46 & 2937.07 & 2863.47 & 3134.10 & 1966.77 \\
\hline
\end{tabular}




\begin{tabular}{|c|c|c|c|c|c|}
\hline 1 & 2 & 3 & 4 & 5 & 6 \\
\hline Krasnodar krai & 1285.55 & 2556.12 & 3090.93 & 2752.25 & 1920.12 \\
\hline Krasnoyarsky krai & 3909.60 & 5460.76 & 5840.64 & 5929.23 & 4267.16 \\
\hline Kurgan oblast & 7019.96 & 2281.47 & 2425.06 & 2243.74 & 1428.42 \\
\hline Kursk oblast & 2781.76 & 2857.02 & 3182.78 & 3146.55 & 2157.87 \\
\hline Leningrad oblast & 3010.10 & 3244.55 & 3796.21 & 3646.83 & 2615.28 \\
\hline Lipetsk oblast & 3810.72 & 4244.52 & 3781.14 & 3343.44 & 2291.47 \\
\hline Magadan oblast & 7934.41 & 6538.18 & 8973.21 & 9431.27 & 6602.05 \\
\hline Moscow oblast & 2924.48 & 3436.46 & 4247.75 & 4432.85 & 3206.78 \\
\hline Murmansk oblast & 5501.87 & 5956.56 & 5405.80 & 5301.73 & 3840.02 \\
\hline $\begin{array}{l}\text { Nenetsky autono- } \\
\text { mous okrug }\end{array}$ & 13062.65 & 13188.45 & 14569.49 & 13557.25 & 10300.99 \\
\hline N. Novgorod oblast & 4006.36 & 4493.89 & 4112.32 & 4546.94 & 2307.04 \\
\hline Novgorod oblast & 2458.18 & 2471.90 & 3250.13 & 3104.27 & 2321.30 \\
\hline Novossibirsk oblast & 2459.79 & 3807.20 & 4117.71 & 4280.05 & 2447.15 \\
\hline Omsk oblast & 2038.17 & 4337.07 & 4593.40 & 4647.42 & 2482.47 \\
\hline Orenburg oblast & 6047.59 & 3254.57 & 3891.47 & 4081.45 & 2220.67 \\
\hline Orel oblast & 2406.00 & 2744.89 & 2836.73 & 2968.93 & 2161.50 \\
\hline Penza oblast & 2020.75 & 1994.05 & 2404.71 & 2313.86 & 1271.73 \\
\hline Perm oblast & 6496.69 & 5134.73 & 4821.36 & 5039.09 & 3059.67 \\
\hline Primorye krai & 3280.51 & 3888.37 & 4155.31 & 4322.39 & 2832.76 \\
\hline Pskov oblast & 2191.50 & 2523.11 & 2630.45 & 2623.89 & 1598.79 \\
\hline Republic Of Adygeya & 1541.02 & 1514.81 & 1647.11 & 1690.96 & 1209.52 \\
\hline Altay Republic & 30122.50 & 2360.87 & 2955.07 & 2941.94 & 1834.80 \\
\hline $\begin{array}{l}\text { Republic of Bashkor- } \\
\text { tostan }\end{array}$ & 1253.11 & 4185.02 & 4655.86 & 4982.87 & 2763.14 \\
\hline Republic of Buryatia & 526.69 & 3061.61 & 3030.59 & 3355.14 & 2079.64 \\
\hline Republic of Dagestan & 961.34 & 846.43 & 1052.66 & 1343.10 & 734.23 \\
\hline Ingoush Republic & 660.00 & 937.83 & 1167.04 & 1342.99 & 788.66 \\
\hline Kalmyk Republic & 1323.75 & 1116.24 & 1338.11 & 1442.20 & 888.44 \\
\hline Republic of Karelia & 4291.89 & 4109.71 & 3796.34 & 3906.14 & 2515.62 \\
\hline Republic Komi & 5569.13 & 6546.87 & 5739.96 & 6870.01 & 4705.95 \\
\hline Republic of Mary El & 2222.72 & 2050.76 & 1917.33 & 2334.91 & 1383.15 \\
\hline $\begin{array}{l}\text { Republic of Mor- } \\
\text { dovia }\end{array}$ & 1964.55 & 2279.43 & 2764.59 & 2676.26 & 1620.12 \\
\hline $\begin{array}{l}\text { Republic of Sakha } \\
\text { (Yakutia) }\end{array}$ & 8175.48 & 8642.44 & 9558.51 & 10104.75 & 7237.44 \\
\hline $\begin{array}{l}\text { Republic of North } \\
\text { Ossetia (Alania) }\end{array}$ & 1336.12 & 1463.68 & 1543.48 & 1464.75 & 1023.29 \\
\hline Republic of Tatarstan & 3232.04 & 4374.36 & 5370.34 & 5112.48 & 3177.37 \\
\hline Republic of Tyva & 7697.40 & 1354.12 & 1423.15 & 1452.33 & 1053.32 \\
\hline
\end{tabular}




\begin{tabular}{|c|c|c|c|c|c|}
\hline 1 & 2 & 3 & 4 & 5 & 6 \\
\hline $\begin{array}{l}\text { Republic of Khakas- } \\
\text { sia }\end{array}$ & 27169.86 & 3622.01 & 3610.20 & 4013.59 & 2320.85 \\
\hline Rostov oblast & 1882.15 & 2587.92 & 2585.02 & 2503.26 & 1592.68 \\
\hline Ryazan oblast & 3204.65 & 2914.94 & 2890.93 & 2986.08 & 1686.42 \\
\hline Samara oblast & 5115.58 & 5911.52 & 6381.94 & 6982.23 & 3829.93 \\
\hline Saratov oblast & 2907.70 & 3242.31 & 3044.95 & 3230.12 & 1707.74 \\
\hline Sakhalin oblast & 4798.81 & 4455.38 & 4790.74 & 5561.00 & 3581.03 \\
\hline Sverdlovsk oblast & 3020.60 & 5903.90 & 5430.62 & 5429.03 & 2960.74 \\
\hline Smolensk oblast & 2878.43 & 2790.17 & 3017.04 & 2981.24 & 1841.71 \\
\hline Stavropol krai & 3544.60 & 2838.97 & 2852.87 & 2779.88 & 1884.59 \\
\hline $\begin{array}{l}\text { Taymyr (Dolgan- } \\
\text { Nenetsky) autono- } \\
\text { mous okrug }\end{array}$ & 1288.86 & 1384.75 & 1568.85 & 1825.55 & 1536.99 \\
\hline Tambov oblast & 2144.87 & 2082.63 & 2103.95 & 2033.00 & 1419.27 \\
\hline Tver oblast & 2890.56 & 3059.62 & 3162.21 & 2996.96 & 2074.50 \\
\hline Tomsk oblast & 30802.78 & 4958.49 & 5324.92 & 5467.77 & 3259.90 \\
\hline Tula oblast & 2683.79 & 2979.26 & 3025.40 & 2912.18 & 1994.18 \\
\hline Tyumen oblast & 1428.19 & 15613.01 & 21848.44 & 20764.34 & 12602.79 \\
\hline Udmurt Republic & 1687.02 & 3303.21 & 3826.23 & 3736.98 & 2296.60 \\
\hline Ulyanovsk oblast & 3087.94 & 3110.60 & 3355.58 & 2998.75 & 1860.92 \\
\hline $\begin{array}{l}\text { Ust-Ordynsky Bury- } \\
\text { atsky autonomous } \\
\text { okrug }\end{array}$ & 458.12 & 449.53 & 414.31 & 414.33 & 1094.49 \\
\hline Khabarovsk krai & 3863.41 & 4361.47 & 5787.19 & 6554.79 & 3927.78 \\
\hline $\begin{array}{l}\text { Khanty-Mansy } \\
\text { autonomous okrug }\end{array}$ & 21955.10 & 26318.35 & 30096.50 & 30339.85 & 19809.48 \\
\hline Chelyabinsk oblast & 2661.10 & 4276.14 & 5423.74 & 5386.35 & 2566.91 \\
\hline Chita oblast & 2058.77 & 3229.44 & 3094.56 & 3097.12 & 1954.88 \\
\hline Chuvash Republic & 2210.07 & 2209.73 & 2422.30 & 2344.22 & 1517.31 \\
\hline $\begin{array}{l}\text { Chukotka autono- } \\
\text { mous okrug }\end{array}$ & 5388.00 & 5910.33 & 7008.65 & 5370.18 & 4089.79 \\
\hline $\begin{array}{l}\text { Evenk autonomous } \\
\text { okrug }\end{array}$ & 1542.71 & 1740.36 & 1922.62 & 2149.90 & 2267.48 \\
\hline $\begin{array}{l}\text { Yamal-Nenetsky } \\
\text { autonomous okrug }\end{array}$ & 17563.90 & 20787.40 & 23681.80 & 24597.81 & 16535.95 \\
\hline Yaroslavl oblast & 4343.06 & 4423.67 & 4363.71 & 4246.41 & 2845.39 \\
\hline
\end{tabular}


Table A1-2

GDP Per Capita in 1994 Prices (as Rb. Th.) over 1999-2002

\begin{tabular}{|c|c|c|c|c|}
\hline & 1999 & 2000 & 2001 & 2002 \\
\hline 1 & 2 & 3 & 4 & 5 \\
\hline $\begin{array}{l}\text { Aginsky Buryatsky autonomous } \\
\text { okrug }\end{array}$ & 864.04 & 1221.60 & 1490.35 & 1809.71 \\
\hline Altay krai & 1712.83 & 2030.95 & 2249.63 & 2365.08 \\
\hline Amur oblast & 3271.62 & 3555.78 & 4374.50 & 5081.24 \\
\hline Arkhangel' oblast & 3778.77 & 4288.61 & 4013.70 & 5644.01 \\
\hline Astarkhan oblast & 2049.30 & 3161.00 & 2971.85 & 3190.69 \\
\hline Belgorod oblast & 2837.66 & 3081.85 & 3126.64 & 3255.61 \\
\hline Bryansk oblast & 1431.85 & 1790.03 & 1868.48 & 2110.37 \\
\hline Vladimir oblast & 2226.36 & 2566.40 & 2738.00 & 2948.98 \\
\hline Volgograd oblast & 2280.53 & 2806.28 & 3061.00 & 3166.63 \\
\hline Vologda oblast & 4719.10 & 6010.03 & 5220.33 & 5592.96 \\
\hline Voronezh oblast & 1902.68 & 2138.46 & 2187.39 & 2711.31 \\
\hline City of Moscow & 9136.04 & 12574.70 & 12958.42 & 11234.47 \\
\hline Saint Petesrburg & 3929.08 & 4390.66 & 5027.78 & 5761.75 \\
\hline Jewish Autonomous oblast & 1998.70 & 2156.55 & 2365.64 & 2800.30 \\
\hline Ivanovo oblast & 1281.96 & 1606.69 & 1656.65 & 1982.55 \\
\hline Irkutsk oblast & 4189.38 & 4428.55 & 4547.72 & 5083.25 \\
\hline Kabardino-Balkar Republic & 1536.75 & 1991.34 & 2775.30 & 1831.12 \\
\hline Kaliningrad oblast & 2264.43 & 2922.16 & 3091.48 & 3647.11 \\
\hline Kaluga oblast & 1998.80 & 2538.10 & 2835.54 & 2907.11 \\
\hline Kamchatka oblast & 4888.28 & 3721.15 & 4213.43 & 5438.63 \\
\hline Karachay-Cherkess Republic & 1321.93 & 1490.84 & 2275.27 & 1891.47 \\
\hline Kemerovo oblast & 3466.17 & 4040.11 & 4274.00 & 4734.68 \\
\hline Kirov oblast & 2385.66 & 2744.32 & 2719.25 & 3034.91 \\
\hline Komi-Permyak autonomous okrug & 1414.23 & 1458.11 & 1518.33 & 1708.88 \\
\hline Koryak autonomous okrug & 7531.86 & 8569.94 & 9541.53 & 10234.64 \\
\hline Kostroma oblast & 2349.54 & 2441.10 & 2741.15 & 2859.81 \\
\hline Krasnodar krai & 2707.65 & 3220.13 & 3307.02 & 3534.40 \\
\hline Krasnoyarsky krai & 5836.25 & 8225.19 & 7326.31 & 6413.69 \\
\hline Kurgan oblast & 1600.54 & 1731.10 & 1764.13 & 2076.92 \\
\hline Kursk oblast & 2103.60 & 2433.05 & 2359.38 & 2567.31 \\
\hline
\end{tabular}




\begin{tabular}{|c|c|c|c|c|}
\hline 1 & 2 & 3 & 4 & 5 \\
\hline Leningrad oblast & 3388.03 & 3955.32 & 4550.30 & 4947.59 \\
\hline Lipetsk oblast & 3012.94 & 3739.00 & 3418.96 & 4241.89 \\
\hline Magadan oblast & 7230.60 & 7386.66 & 7969.04 & 11937.98 \\
\hline Moscow oblast & 3646.69 & 3918.11 & 4285.02 & 4886.51 \\
\hline Murmansk oblast & 5203.60 & 5810.07 & 4912.14 & 5071.51 \\
\hline Nenetsky autonomous okrug & 9554.20 & 27411.16 & 22901.86 & 26597.28 \\
\hline N. Novgorod oblast & 2481.05 & 2932.48 & 3700.09 & 3844.12 \\
\hline Novgorod oblast & 2967.22 & 3284.04 & 3694.95 & 3750.82 \\
\hline Novossibirsk oblast & 2952.08 & 3368.11 & 4131.63 & 4405.77 \\
\hline Omsk oblast & 2399.53 & 2592.99 & 3252.61 & 3778.37 \\
\hline Orenburg oblast & 3032.82 & 3763.64 & 3636.07 & 3703.24 \\
\hline Orel oblast & 2739.72 & 3300.56 & 3458.41 & 3906.83 \\
\hline Penza oblast & 1482.97 & 1727.73 & 1857.22 & 2168.44 \\
\hline Perm oblast & 3924.01 & 4324.73 & 5097.17 & 4809.45 \\
\hline Primorye krai & 3777.48 & 3736.05 & 3666.25 & 4540.78 \\
\hline Pskov oblast & 2098.86 & 2576.70 & 2647.87 & 2876.78 \\
\hline Republic Of Adygeya & 1192.11 & 1218.07 & 1146.01 & 1230.55 \\
\hline Altay Republic & 2136.52 & 2515.89 & 3351.43 & 3265.01 \\
\hline Republic of Bashkortostan & 3443.95 & 4065.51 & 4056.45 & 4065.38 \\
\hline Republic of Buryatia & 2159.86 & 2312.01 & 2729.69 & 3256.95 \\
\hline Republic of Dagestan & 801.03 & 1133.73 & 1431.31 & 1413.14 \\
\hline Ingoush Republic & 641.83 & 1561.77 & 948.21 & 622.81 \\
\hline Kalmyk Republic & 808.98 & 2656.40 & 1220.92 & 3235.86 \\
\hline Republic of Karelia & 3468.01 & 4096.16 & 4189.20 & 4682.23 \\
\hline Republic Komi & 5839.44 & 6786.01 & 7750.20 & 7843.30 \\
\hline Republic of Mary El & 1408.60 & 1410.73 & 1543.15 & 1687.80 \\
\hline Republic of Mordovia & 1754.55 & 2400.86 & 2069.03 & 1792.82 \\
\hline Republic of Sakha (Yakutia) & 9926.99 & 11038.33 & 12056.46 & 12815.46 \\
\hline Republic of North Ossetia (Alania) & 1357.21 & 1531.71 & 3009.83 & 1792.45 \\
\hline Republic of Tatarstan & 3790.29 & 5307.24 & 4856.68 & 4991.94 \\
\hline Republic of Tyva & 1099.64 & 1231.78 & 1482.55 & 1836.36 \\
\hline Republic of Khakassia & 2682.49 & 2819.76 & 2738.84 & 3117.80 \\
\hline Rostov oblast & 1864.96 & 2232.24 & 2440.10 & 2354.53 \\
\hline Ryazan oblast & 1961.71 & 2324.99 & 2733.51 & 3100.74 \\
\hline
\end{tabular}




\begin{tabular}{|c|c|c|c|c|}
\hline 1 & 2 & 3 & 4 & 5 \\
\hline Samara oblast & 4626.19 & 5140.15 & 5821.11 & 5827.92 \\
\hline Saratov oblast & 2007.25 & 2420.88 & 2709.87 & 2816.23 \\
\hline Sakhalin oblast & 5395.78 & 6130.02 & 7063.70 & 7524.81 \\
\hline Sverdlovsk oblast & 3389.41 & 3799.43 & 4121.21 & 4202.11 \\
\hline Smolensk oblast & 2301.12 & 2648.04 & 2971.79 & 3262.63 \\
\hline Stavropol krai & 1844.39 & 2220.93 & 2359.64 & 2306.82 \\
\hline $\begin{array}{l}\text { Taymyr (Dolgan-Nenetsky) } \\
\text { autonomous okrug }\end{array}$ & 1901.58 & 4619.89 & 5203.64 & 3800.43 \\
\hline Tambov oblast & 1688.73 & 1973.51 & 2308.50 & 2498.21 \\
\hline Tver oblast & 2354.93 & 2651.99 & 3029.88 & 3192.16 \\
\hline Tomsk oblast & 3256.30 & 4101.83 & 4922.44 & 5409.01 \\
\hline Tula oblast & 2337.02 & 2856.27 & 3122.18 & 3358.72 \\
\hline Tyumen oblast & 37185.51 & 4576.27 & 5313.77 & 63690.17 \\
\hline Udmurt Republic & 2979.29 & 3698.50 & 3809.37 & 4010.74 \\
\hline Ulyanovsk oblast & 2040.90 & 2151.30 & 2100.03 & 2218.63 \\
\hline $\begin{array}{l}\text { Ust-Ordynsky Buryatsky autono- } \\
\text { mous okrug }\end{array}$ & 1193.59 & 1772.88 & 2235.44 & 2645.96 \\
\hline Khabarovsk krai & 4084.43 & 5611.49 & 5523.30 & 6031.98 \\
\hline Khanty-Mansy autonomous okrug & 24350.95 & 36972.71 & 39073.32 & 34292.78 \\
\hline Chelyabinsk oblast & 3281.65 & 4340.67 & 3919.32 & 4263.29 \\
\hline Chita oblast & 2384.71 & 2833.19 & 2885.21 & 3410.90 \\
\hline Chuvash Republic & 1606.54 & 1774.39 & 2007.88 & 2076.56 \\
\hline Chukotka autonomous okrug & 3351.56 & 3638.68 & 6479.08 & 10236.66 \\
\hline Evenk autonomous okrug & 3017.81 & 4484.62 & 5338.23 & 4217.79 \\
\hline $\begin{array}{l}\text { Yamal-Nenetsky autonomous } \\
\text { okrug }\end{array}$ & 20906.77 & 32443.54 & 38672.98 & 50581.56 \\
\hline Yaroslavl oblast & 3468.09 & 3741.23 & 4775.80 & 5109.31 \\
\hline
\end{tabular}


Table A1-3

Main Statistical Indicators Characterizing the Level of Inter-Regional
Disparity by GDP Per Capita in 1994 Prices over 1994-1998

\begin{tabular}{|c|c|c|c|c|c|}
\hline & 1994 & 1995 & 1996 & 1997 & 1998 \\
\hline Average value & 4538.58 & 4124.38 & 4567.54 & 4610.18 & 3018.30 \\
\hline Average weighted & 3780.14 & 4378.81 & 4951.05 & 5086.36 & 3133.67 \\
\hline Dispersion & 32059402.75 & 14214829.06 & 20094120.98 & 20326935.90 & 9196929.10 \\
\hline Dispersion weighted & 31887409.51 & 14097470.14 & 19902251.11 & 20122810.23 & 9117112.49 \\
\hline Variation coefficient & 1.248 & 0.914 & 0.981 & 0.978 & 1.005 \\
\hline $\begin{array}{l}\text { Variation coefficient } \\
\text { (weighted) - method } 1\end{array}$ & 1.244 & 0.910 & 0.977 & 0.973 & 1.000 \\
\hline $\begin{array}{l}\text { Variation coefficient } \\
\text { (weighted) - method } 2\end{array}$ & 1.007 & 0.730 & 0.824 & 0.823 & 0.839 \\
\hline Index Gini & 0.478 & 0.236 & 0.240 & 0.244 & 0.285 \\
\hline Index Tale & 0.044 & 0.026 & 0.029 & 0.029 & 0.030 \\
\hline Median & 2867.05 & 3119.03 & 3293.81 & 3281.92 & 2159.68 \\
\hline
\end{tabular}

Table A1-4

Main Statistical Indicators Characterizing the Level of Inter-Regional Disparity by GDP Per Capita in 1994 Prices over 1999-2002

\begin{tabular}{lcccc}
\hline & $\mathbf{1 9 9 9}$ & $\mathbf{2 0 0 0}$ & $\mathbf{2 0 0 1}$ & $\mathbf{2 0 0 2}$ \\
\hline Average value & 3820.46 & 4459.18 & 4764.78 & 5831.56 \\
Average weighted & 4351.38 & 4474.66 & 4747.45 & 5432.77 \\
Dispersion & 24717227.74 & 31742084.34 & 35559245.50 & 83159163.54 \\
$\begin{array}{l}\text { Dispersion weighted } \\
\text { Variation coefficient }\end{array}$ & 24499713.46 & 31514787.63 & 35298071.79 & 82550248.27 \\
$\begin{array}{l}\text { Variation coefficient } \\
\text { (weighted) - method 1 }\end{array}$ & 1.301 & 1.263 & 1.252 & 1.564 \\
$\begin{array}{l}\text { Variation coefficient } \\
\text { (weighted)- method 2 }\end{array}$ & 1.296 & 1.259 & 1.247 & 1.558 \\
Index Gini & & & & \\
Index Tale & 0.340 & 0.990 & 1.002 & 1.348 \\
Median & 0.041 & 0.330 & 0.338 & 0.411 \\
\hline
\end{tabular}




\section{Accumulated and Expressed in Annual Terms Regional GRP Per Capita Growth Rate in 1994 Prices}

\begin{tabular}{|c|c|c|}
\hline & $\begin{array}{c}\text { Accumulated GRP growth rate } \\
\text { over } \\
1994-2002 \text { гг. as \% } \\
\end{array}$ & $\begin{array}{l}\text { The average annual GRP growth } \\
\text { rate over } \\
\text { 1994-2002 гг. as \%/a. } \\
\end{array}$ \\
\hline 1 & 2 & 3 \\
\hline $\begin{array}{l}\text { Aginsky Buryatsky autonomous } \\
\text { okrug }\end{array}$ & 73.02 & 9.13 \\
\hline Altay krai & -53.03 & -6.63 \\
\hline Amur oblast & 23.82 & 2.98 \\
\hline Arkhangel' oblast & 61.37 & 7.67 \\
\hline Astarkhan oblast & 51.63 & 6.45 \\
\hline Belgorod oblast & 16.08 & 2.01 \\
\hline Bryansk oblast & -5.88 & -0.73 \\
\hline Vladimir oblast & 16.01 & 2.00 \\
\hline Volgograd oblast & -0.65 & -0.08 \\
\hline Vologda oblast & 22.92 & 2.87 \\
\hline Voronezh oblast & 15.20 & 1.90 \\
\hline City of Moscow & 81.09 & 10.14 \\
\hline Saint Petesrburg & 64.31 & 8.04 \\
\hline Jewish Autonomous oblast & -1.94 & -0.24 \\
\hline Ivanovo oblast & -3.27 & -0.41 \\
\hline Irkutsk oblast & 239.77 & 29.97 \\
\hline Kabardino-Balkar Republic & 59.97 & 7.50 \\
\hline Kaliningrad oblast & 46.92 & 5.86 \\
\hline Kaluga oblast & 6.18 & 0.77 \\
\hline Kamchatka oblast & 6.89 & 0.86 \\
\hline Karachay-Cherkess Republic & 17.23 & 2.15 \\
\hline Kemerovo oblast & 64.22 & 8.03 \\
\hline Kirov oblast & 14.47 & 1.81 \\
\hline $\begin{array}{l}\text { Komi-Permyak autonomous } \\
\text { okrug }\end{array}$ & -18.70 & -2.34 \\
\hline Koryak autonomous okrug & 24.90 & 3.11 \\
\hline Kostroma oblast & 1.61 & 0.20 \\
\hline Krasnodar krai & 174.93 & 21.87 \\
\hline Krasnoyarsky krai & 64.05 & 8.01 \\
\hline
\end{tabular}




\begin{tabular}{|c|c|c|}
\hline 1 & 2 & 3 \\
\hline Kurgan oblast & -70.41 & -8.80 \\
\hline Kursk oblast & -7.71 & -0.96 \\
\hline Leningrad oblast & 64.37 & 8.05 \\
\hline Lipetsk oblast & 11.31 & 1.41 \\
\hline Magadan oblast & 50.46 & 6.31 \\
\hline Moscow oblast & 67.09 & 8.39 \\
\hline Murmansk oblast & -7.82 & -0.98 \\
\hline Nenetsky autonomous okrug & 103.61 & 12.95 \\
\hline N. Novgorod oblast & -4.05 & -0.51 \\
\hline Novgorod oblast & 52.59 & 6.57 \\
\hline Novossibirsk oblast & 79.11 & 9.89 \\
\hline Omsk oblast & 85.38 & 10.67 \\
\hline Orenburg oblast & -38.77 & -4.85 \\
\hline Orel oblast & 62.38 & 7.80 \\
\hline Penza oblast & 7.31 & 0.91 \\
\hline Perm oblast & -25.97 & -3.25 \\
\hline Primorye krai & 38.42 & 4.80 \\
\hline Pskov oblast & 31.27 & 3.91 \\
\hline Republic Of Adygeya & -20.15 & -2.52 \\
\hline Altay Republic & -89.16 & -11.15 \\
\hline Republic of Bashkortostan & 224.42 & 28.05 \\
\hline Republic of Buryatia & 518.39 & 64.80 \\
\hline Republic of Dagestan & 47.00 & 5.87 \\
\hline Ingoush Republic & -5.63 & -0.70 \\
\hline Kalmyk Republic & 144.45 & 18.06 \\
\hline Republic of Karelia & 9.09 & 1.14 \\
\hline Republic Komi & 40.84 & 5.10 \\
\hline Republic of Mary El & -24.07 & -3.01 \\
\hline Republic of Mordovia & -8.74 & -1.09 \\
\hline Republic of Sakha (Yakutia) & 56.75 & 7.09 \\
\hline $\begin{array}{l}\text { Republic of North Ossetia } \\
\text { (Alania) }\end{array}$ & 34.15 & 4.27 \\
\hline Republic of Tatarstan & 54.45 & 6.81 \\
\hline Republic of Tyva & -76.14 & -9.52 \\
\hline Republic of Khakassia & -88.52 & -11.07 \\
\hline
\end{tabular}




\begin{tabular}{|c|c|c|}
\hline 1 & 2 & 3 \\
\hline Rostov oblast & 25.10 & 3.14 \\
\hline Ryazan oblast & -3.24 & -0.41 \\
\hline Samara oblast & 13.92 & 1.74 \\
\hline Saratov oblast & -3.15 & -0.39 \\
\hline Sakhalin oblast & 56.81 & 7.10 \\
\hline Sverdlovsk oblast & 39.11 & 4.89 \\
\hline Smolensk oblast & 13.35 & 1.67 \\
\hline Stavropol krai & -34.92 & -4.37 \\
\hline $\begin{array}{l}\text { Taymyr (Dolgan-Nenetsky) } \\
\text { autonomous okrug }\end{array}$ & 194.87 & 24.36 \\
\hline Tambov oblast & 16.47 & 2.06 \\
\hline Tver oblast & 10.43 & 1.30 \\
\hline Tomsk oblast & -82.44 & -10.30 \\
\hline Tula oblast & 25.15 & 3.14 \\
\hline Tyumen oblast & 4359.51 & 544.94 \\
\hline Udmurt Republic & 137.74 & 17.22 \\
\hline Ulyanovsk oblast & -28.15 & -3.52 \\
\hline $\begin{array}{l}\text { Ust-Ordynsky Buryatsky autono- } \\
\text { mous okrug }\end{array}$ & 477.57 & 59.70 \\
\hline Khabarovsk krai & 56.13 & 7.02 \\
\hline Khanty-Mansy autonomous okrug & 56.20 & 7.02 \\
\hline Chelyabinsk oblast & 60.21 & 7.53 \\
\hline Chita oblast & 65.68 & 8.21 \\
\hline Chuvash Republic & -6.04 & -0.76 \\
\hline Chukotka autonomous okrug & 89.99 & 11.25 \\
\hline Evenk autonomous okrug & 173.40 & 21.68 \\
\hline $\begin{array}{l}\text { Yamal-Nenetsky autonomous } \\
\text { okrug }\end{array}$ & 187.99 & 23.50 \\
\hline Yaroslavl oblast & 17.64 & 2.21 \\
\hline
\end{tabular}


Financial Aid to Regions' GRP=s Ratio in 1994-1998, as \%

\begin{tabular}{|c|c|c|c|c|c|}
\hline & 1994 & 1995 & 1996 & 1997 & 1998 \\
\hline 1 & 2 & 3 & 4 & 5 & 6 \\
\hline $\begin{array}{l}\text { Aginsky Buryatsky autono- } \\
\text { mous okrug }\end{array}$ & 48.52 & 17.59 & 24.14 & 25.46 & 27.30 \\
\hline Altay krai & 5.44 & 6.70 & 8.81 & 10.69 & 9.09 \\
\hline Amur oblast & 6.51 & 5.53 & 10.33 & 9.19 & 7.54 \\
\hline Arkhangel' oblast & 4.49 & 4.25 & 2.85 & 2.07 & 1.81 \\
\hline Astarkhan oblast & 7.96 & 3.60 & 7.08 & 6.72 & 4.71 \\
\hline Belgorod oblast & 3.53 & 0.98 & 1.32 & 1.01 & 1.62 \\
\hline Bryansk oblast & 4.33 & 3.97 & 4.78 & 3.06 & 3.56 \\
\hline Vladimir oblast & 4.35 & 1.71 & 5.49 & 4.75 & 4.42 \\
\hline Volgograd oblast & 2.50 & 0.81 & 2.06 & 1.27 & 2.35 \\
\hline Vologda oblast & 2.17 & 0.94 & 2.54 & 2.43 & 0.49 \\
\hline Voronezh oblast & 2.94 & 1.55 & 2.88 & 2.75 & 3.09 \\
\hline City of Moscow & 5.80 & 1.56 & 1.13 & 0.79 & 0.18 \\
\hline Saint Petesrburg & 1.53 & 0.24 & 0.53 & 1.13 & 0.12 \\
\hline Jewish Autonomous oblast & 10.90 & 14.29 & 12.62 & 16.65 & 17.32 \\
\hline Ivanovo oblast & 6.02 & 7.13 & 9.93 & 13.87 & 5.92 \\
\hline Irkutsk oblast & 7.66 & 0.91 & 1.23 & 0.67 & 0.90 \\
\hline Kabardino-Balkar Republic & 35.07 & 12.02 & 14.58 & 19.54 & 19.01 \\
\hline Kaliningrad oblast & 5.20 & 2.45 & 4.30 & 3.71 & 1.89 \\
\hline Kaluga oblast & 5.35 & 3.07 & 7.27 & 6.26 & 6.58 \\
\hline Kamchatka oblast & 18.16 & 7.82 & 5.16 & 10.74 & 6.55 \\
\hline Karachay-Cherkess Republic & 21.32 & 7.90 & 8.73 & 13.33 & 11.12 \\
\hline Kemerovo oblast & 9.25 & 2.67 & 5.01 & 3.26 & 3.57 \\
\hline Kirov oblast & 3.44 & 1.43 & 3.56 & 4.14 & 4.83 \\
\hline $\begin{array}{l}\text { Komi-Permyak autonomous } \\
\text { okrug }\end{array}$ & 24.90 & 8.67 & 12.51 & 10.01 & 22.09 \\
\hline Koryak autonomous okrug & 78.18 & 42.51 & 23.23 & 18.81 & 14.11 \\
\hline Kostroma oblast & 8.43 & 9.92 & 18.41 & 9.60 & 6.77 \\
\hline Krasnodar krai & 8.54 & 1.64 & 2.87 & 4.41 & 2.40 \\
\hline Krasnoyarsky krai & 2.52 & 0.13 & 0.52 & 0.49 & 0.04 \\
\hline Kurgan oblast & 2.42 & 2.13 & 5.51 & 4.82 & 6.01 \\
\hline Kursk oblast & 2.36 & 1.87 & 1.82 & 4.58 & 2.39 \\
\hline
\end{tabular}




\begin{tabular}{|c|c|c|c|c|c|}
\hline 1 & 2 & 3 & 4 & 5 & 6 \\
\hline Leningrad oblast & 2.91 & 1.74 & 0.93 & 2.26 & 1.13 \\
\hline Lipetsk oblast & 1.87 & 0.29 & 0.22 & 0.31 & 0.33 \\
\hline Magadan oblast & 18.76 & 14.07 & 15.56 & 12.81 & 7.79 \\
\hline Moscow oblast & 3.18 & 1.81 & 1.99 & 2.13 & 0.46 \\
\hline Murmansk oblast & 3.40 & 2.58 & 4.98 & 8.21 & 5.19 \\
\hline Nenetsky autonomous okrug & 4.73 & 2.49 & 4.33 & 7.07 & 2.68 \\
\hline N. Novgorod oblast & 0.87 & 0.14 & 1.52 & 0.91 & 0.74 \\
\hline Novgorod oblast & 7.03 & 5.97 & 6.44 & 8.85 & 6.61 \\
\hline Novossibirsk oblast & 4.52 & 1.15 & 1.94 & 2.95 & 0.96 \\
\hline Omsk oblast & 7.98 & 2.01 & 3.20 & 1.62 & 2.15 \\
\hline Orenburg oblast & 1.99 & 1.10 & 1.78 & 1.60 & 3.79 \\
\hline Orel oblast & 8.24 & 6.00 & 7.34 & 10.60 & 3.83 \\
\hline Penza oblast & 8.66 & 3.36 & 5.48 & 4.27 & 4.16 \\
\hline Perm oblast & 1.07 & 0.70 & 1.37 & 1.22 & 0.26 \\
\hline Primorye krai & 7.89 & 5.35 & 4.57 & 7.73 & 5.84 \\
\hline Pskov oblast & 6.41 & 4.23 & 11.60 & 12.22 & 7.87 \\
\hline Republic Of Adygeya & 15.90 & 6.47 & 13.25 & 16.47 & 17.55 \\
\hline Altay Republic & 2.78 & 22.31 & 19.22 & 18.95 & 18.26 \\
\hline Republic of Bashkortostan & 2.05 & 0.00 & 0.01 & 0.00 & 1.04 \\
\hline Republic of Buryatia & 49.54 & 6.85 & 5.97 & 7.90 & 8.92 \\
\hline Republic of Dagestan & 61.27 & 36.46 & 21.43 & 24.44 & 29.60 \\
\hline Ingoush Republic & 63.94 & 17.68 & 23.12 & 25.98 & 49.40 \\
\hline Kalmyk Republic & 50.24 & 29.74 & 28.75 & 27.31 & 29.89 \\
\hline Republic of Karelia & 2.78 & 2.86 & 4.18 & 3.85 & 2.67 \\
\hline Republic Komi & 4.43 & 2.65 & 4.02 & 1.59 & 0.80 \\
\hline Republic of Mary El & 11.31 & 7.32 & 8.70 & 7.38 & 4.71 \\
\hline Republic of Mordovia & 8.32 & 6.27 & 9.73 & 9.32 & 7.14 \\
\hline Republic of Sakha (Yakutia) & 0.77 & 0.19 & 7.36 & 9.07 & 2.64 \\
\hline $\begin{array}{l}\text { Republic of North Ossetia } \\
\text { (Alania) }\end{array}$ & 27.36 & 13.38 & 19.84 & 18.12 & 16.24 \\
\hline Republic of Tatarstan & 2.35 & 0.28 & 0.48 & 0.44 & 1.11 \\
\hline Republic of Tyva & 15.19 & 40.51 & 33.01 & 30.00 & 33.75 \\
\hline Republic of Khakassia & 0.66 & 1.71 & 2.81 & 2.01 & 2.02 \\
\hline Rostov oblast & 3.66 & 1.51 & 5.43 & 3.61 & 3.19 \\
\hline Ryazan oblast & 4.23 & 0.50 & 3.38 & 4.00 & 2.73 \\
\hline
\end{tabular}




\begin{tabular}{|c|c|c|c|c|c|}
\hline 1 & 2 & 3 & 4 & 5 & 6 \\
\hline Samara oblast & 1.47 & 0.20 & 0.22 & 0.29 & 0.51 \\
\hline Saratov oblast & 2.91 & 1.48 & 3.16 & 3.18 & 4.47 \\
\hline Sakhalin oblast & 8.21 & 7.08 & 7.87 & 7.72 & 4.97 \\
\hline Sverdlovsk oblast & 1.76 & 0.71 & 0.30 & 0.87 & 0.40 \\
\hline Smolensk oblast & 2.90 & 1.06 & 2.50 & 2.73 & 3.59 \\
\hline Stavropol krai & 5.42 & 3.07 & 4.74 & 4.32 & 4.03 \\
\hline $\begin{array}{l}\text { Taymyr (Dolgan-Nenetsky) } \\
\text { autonomous okrug }\end{array}$ & 94.67 & 35.36 & 39.07 & 39.88 & 19.67 \\
\hline Tambov oblast & 5.61 & 3.54 & 4.88 & 5.62 & 5.29 \\
\hline Tver oblast & 3.75 & 1.98 & 4.57 & 5.37 & 2.45 \\
\hline Tomsk oblast & 0.68 & 1.66 & 2.18 & 2.82 & 1.77 \\
\hline Tula oblast & 3.81 & 1.37 & 5.37 & 5.14 & 2.33 \\
\hline Tyumen oblast & 7.05 & 0.17 & 0.31 & 0.35 & 0.31 \\
\hline Udmurt Republic & 7.88 & 1.63 & 4.75 & 6.12 & 4.61 \\
\hline Ulyanovsk oblast & 5.78 & 1.75 & 2.33 & 2.13 & 3.04 \\
\hline $\begin{array}{l}\text { Ust-Ordynsky Buryatsky } \\
\text { autonomous okrug }\end{array}$ & 117.07 & 83.21 & 102.30 & 84.85 & 27.62 \\
\hline Khabarovsk krai & 10.11 & 5.40 & 5.35 & 4.83 & 3.33 \\
\hline $\begin{array}{l}\text { Khanty-Mansy autonomous } \\
\text { okrug }\end{array}$ & 0.32 & 0.16 & 0.56 & 0.01 & 0.22 \\
\hline Chelyabinsk oblast & 2.61 & 1.16 & 1.86 & 2.58 & 0.84 \\
\hline Chita oblast & 10.63 & 2.42 & 3.91 & 5.11 & 4.74 \\
\hline Chuvash Republic & 3.69 & 2.70 & 4.03 & 5.71 & 2.58 \\
\hline Chukotka autonomous okrug & 79.04 & 31.11 & 19.80 & 35.61 & 21.09 \\
\hline Evenk autonomous okrug & 157.51 & 134.49 & 90.62 & 165.58 & 71.03 \\
\hline $\begin{array}{l}\text { Yamal-Nenetsky autonomous } \\
\text { okrug }\end{array}$ & 0.71 & 0.23 & 0.50 & 0.03 & 0.06 \\
\hline Yaroslavl oblast & 1.56 & 0.47 & 3.54 & 3.83 & 1.45 \\
\hline
\end{tabular}


Table A1-7

Financial Aid to Regions' GRP=s Ratio in 1999-2002, as \%

\begin{tabular}{|c|c|c|c|c|c|}
\hline & 1999 & 2000 & 2001 & 2002 & $\begin{array}{c}\text { Average over } \\
1994-2002 \\
\end{array}$ \\
\hline 1 & 2 & 3 & 4 & 5 & 6 \\
\hline $\begin{array}{l}\text { Aginsky Buryatsky } \\
\text { autonomous okrug }\end{array}$ & 20.28 & 34.91 & 35.37 & 39.53 & 30.34 \\
\hline Altay krai & 8.33 & 8.35 & 11.40 & 13.57 & 9.15 \\
\hline Amur oblast & 5.60 & 7.33 & 10.19 & 9.71 & 7.99 \\
\hline Arkhangel' oblast & 2.43 & 2.93 & 6.04 & 5.22 & 3.56 \\
\hline Astarkhan oblast & 2.95 & 2.00 & 1.96 & 2.77 & 4.42 \\
\hline Belgorod oblast & 0.35 & 0.83 & 1.53 & 2.51 & 1.52 \\
\hline Bryansk oblast & 3.87 & 7.25 & 9.53 & 8.34 & 5.41 \\
\hline Vladimir oblast & 2.26 & 3.18 & 4.59 & 5.41 & 4.02 \\
\hline Volgograd oblast & 1.59 & 0.76 & 2.22 & 2.12 & 1.74 \\
\hline Vologda oblast & 0.43 & 0.05 & 1.43 & 1.86 & 1.37 \\
\hline Voronezh oblast & 2.16 & 2.17 & 4.54 & 4.54 & 2.96 \\
\hline City of Moscow & 0.00 & 0.00 & 0.43 & -2.74 & 0.79 \\
\hline Saint Petesrburg & 0.09 & 0.07 & 0.86 & 1.43 & 0.67 \\
\hline $\begin{array}{l}\text { Jewish Autonomous } \\
\text { oblast }\end{array}$ & 12.69 & 17.83 & 28.87 & 23.87 & 17.23 \\
\hline Ivanovo oblast & 6.70 & 9.09 & 12.16 & 13.57 & 9.38 \\
\hline Irkutsk oblast & 1.20 & 0.90 & 1.93 & 1.78 & 1.91 \\
\hline $\begin{array}{l}\text { Kabardino-Balkar } \\
\text { Republic }\end{array}$ & 15.28 & 10.80 & 12.88 & 17.53 & 17.41 \\
\hline Kaliningrad oblast & 1.76 & 2.17 & 3.60 & 4.39 & 3.28 \\
\hline Kaluga oblast & 2.58 & 4.58 & 6.76 & 7.83 & 5.59 \\
\hline Kamchatka oblast & 8.84 & 14.67 & 11.20 & 13.52 & 10.74 \\
\hline $\begin{array}{l}\text { Karachay-Cherkess } \\
\text { Republic }\end{array}$ & 11.66 & 13.53 & 17.58 & 26.45 & 14.62 \\
\hline Kemerovo oblast & 2.07 & 2.14 & 3.42 & 3.37 & 3.86 \\
\hline Kirov oblast & 2.39 & 1.66 & 5.34 & 5.77 & 3.62 \\
\hline $\begin{array}{l}\text { Komi-Permyak } \\
\text { autonomous okrug }\end{array}$ & 13.82 & 25.70 & 38.29 & 44.53 & 22.28 \\
\hline $\begin{array}{l}\text { Koryak autonomous } \\
\text { okrug }\end{array}$ & 29.12 & 21.75 & 22.74 & 30.23 & 31.19 \\
\hline Kostroma oblast & 3.57 & 3.82 & 4.94 & 8.15 & 8.18 \\
\hline Krasnodar krai & 1.18 & 1.35 & 2.55 & 4.74 & 3.30 \\
\hline Krasnoyarsky krai & 0.49 & 0.07 & 0.75 & 0.96 & 0.66 \\
\hline Kurgan oblast & 3.63 & 3.96 & 8.44 & 11.54 & 5.38 \\
\hline
\end{tabular}




\begin{tabular}{|c|c|c|c|c|c|}
\hline 1 & 2 & 3 & 4 & 5 & 6 \\
\hline Kursk oblast & 1.75 & 1.91 & 3.06 & 4.14 & 2.65 \\
\hline Leningrad oblast & 0.64 & 0.36 & 1.83 & 1.87 & 1.52 \\
\hline Lipetsk oblast & 0.27 & 0.10 & 0.94 & 0.89 & 0.58 \\
\hline Magadan oblast & 9.22 & 11.06 & 15.30 & 15.67 & 13.36 \\
\hline Moscow oblast & 0.48 & 0.20 & 1.23 & 1.23 & 1.41 \\
\hline Murmansk oblast & 2.75 & 3.27 & 1.51 & 2.26 & 3.79 \\
\hline $\begin{array}{l}\text { Nenetsky autono- } \\
\text { mous okrug }\end{array}$ & 4.78 & 1.22 & 1.84 & 0.71 & 3.32 \\
\hline N. Novgorod oblast & 0.40 & 0.36 & 1.08 & 1.37 & 0.82 \\
\hline Novgorod oblast & 2.14 & 4.51 & 3.00 & 3.53 & 5.34 \\
\hline Novossibirsk oblast & 1.15 & 1.64 & 3.23 & 3.78 & 2.37 \\
\hline Omsk oblast & 1.82 & 2.69 & 4.31 & 4.96 & 3.42 \\
\hline Orenburg oblast & 0.87 & 0.76 & 1.18 & 1.95 & 1.67 \\
\hline Orel oblast & 3.13 & 5.14 & 4.06 & 4.96 & 5.92 \\
\hline Penza oblast & 3.80 & 4.43 & 7.63 & 9.70 & 5.72 \\
\hline Perm oblast & 0.05 & 0.27 & 0.60 & 0.74 & 0.70 \\
\hline Primorye krai & 4.49 & 4.72 & 8.62 & 7.27 & 6.28 \\
\hline Pskov oblast & 5.50 & 8.46 & 9.73 & 13.06 & 8.79 \\
\hline $\begin{array}{l}\text { Republic Of Ady- } \\
\text { geya }\end{array}$ & 13.13 & 11.50 & 18.61 & 31.12 & 16.00 \\
\hline Altay Republic & 8.88 & 15.26 & 26.58 & 36.20 & 18.72 \\
\hline $\begin{array}{l}\text { Republic of Bashkor- } \\
\text { tostan }\end{array}$ & 0.05 & 1.91 & 4.83 & 4.04 & 1.55 \\
\hline Republic of Buryatia & 8.77 & 8.82 & 11.47 & 15.60 & 13.76 \\
\hline Republic of Dagestan & 25.32 & 34.54 & 30.08 & 29.68 & 32.54 \\
\hline Ingoush Republic & 27.27 & 16.82 & 37.82 & 114.03 & 41.78 \\
\hline Kalmyk Republic & 13.62 & 3.62 & 7.83 & 11.67 & 22.52 \\
\hline Republic of Karelia & 2.16 & 2.00 & 3.58 & 5.20 & 3.25 \\
\hline Republic Komi & 0.33 & 0.16 & 0.71 & 1.48 & 1.80 \\
\hline Republic of Mary El & 5.84 & 5.75 & 9.97 & 13.19 & 8.24 \\
\hline $\begin{array}{l}\text { Republic of Mor- } \\
\text { dovia }\end{array}$ & 7.07 & 4.89 & 9.09 & 13.53 & 8.37 \\
\hline $\begin{array}{l}\text { Republic of Sakha } \\
\text { (Yakutia) }\end{array}$ & 5.16 & 4.22 & 9.91 & 8.96 & 5.36 \\
\hline $\begin{array}{l}\text { Republic of North } \\
\text { Ossetia (Alania) }\end{array}$ & 12.25 & 11.17 & 17.09 & 22.22 & 17.52 \\
\hline Republic of Tatarstan & 0.18 & 0.20 & 6.75 & 5.42 & 1.91 \\
\hline Republic of Tyva & 30.65 & 37.70 & 54.77 & 56.65 & 36.91 \\
\hline
\end{tabular}




\begin{tabular}{|c|c|c|c|c|c|}
\hline 1 & 2 & 3 & 4 & 5 & 6 \\
\hline $\begin{array}{l}\text { Republic of Khakas- } \\
\text { sia }\end{array}$ & 2.36 & 1.69 & 3.51 & 4.06 & 2.31 \\
\hline Rostov oblast & 2.19 & 2.61 & 4.27 & 5.84 & 3.59 \\
\hline Ryazan oblast & 1.97 & 1.97 & 3.90 & 3.08 & 2.86 \\
\hline Samara oblast & 0.02 & 0.07 & 0.52 & 0.67 & 0.44 \\
\hline Saratov oblast & 1.95 & 1.66 & 2.77 & 3.70 & 2.81 \\
\hline Sakhalin oblast & 5.04 & 5.85 & 4.92 & 5.12 & 6.31 \\
\hline Sverdlovsk oblast & 0.44 & 0.21 & 0.84 & 0.92 & 0.72 \\
\hline Smolensk oblast & 1.27 & 3.50 & 2.59 & 3.22 & 2.60 \\
\hline Stavropol krai & 2.25 & 3.54 & 4.59 & 8.48 & 4.49 \\
\hline $\begin{array}{l}\text { Taymyr (Dolgan- } \\
\text { Nenetsky) autono- } \\
\text { mous okrug }\end{array}$ & 54.63 & 19.91 & 34.09 & 42.21 & 42.16 \\
\hline Tambov oblast & 3.12 & 4.21 & 6.52 & 8.15 & 5.22 \\
\hline Tver oblast & 1.90 & 4.61 & 4.62 & 4.43 & 3.74 \\
\hline Tomsk oblast & 1.26 & 1.61 & 1.51 & 2.68 & 1.80 \\
\hline Tula oblast & 1.46 & 2.33 & 3.39 & 3.65 & 3.20 \\
\hline Tyumen oblast & 0.14 & 0.09 & 0.99 & 0.12 & 1.06 \\
\hline Udmurt Republic & 1.87 & 1.17 & 2.08 & 3.07 & 3.69 \\
\hline Ulyanovsk oblast & 1.33 & 0.95 & 4.24 & 5.54 & 3.01 \\
\hline $\begin{array}{l}\text { Ust-Ordynsky Bury- } \\
\text { atsky autonomous } \\
\text { okrug }\end{array}$ & 20.81 & 25.18 & 28.51 & 36.86 & 58.49 \\
\hline Khabarovsk krai & 4.54 & 3.13 & 5.15 & 4.74 & 5.18 \\
\hline $\begin{array}{l}\text { Khanty-Mansy } \\
\text { autonomous okrug }\end{array}$ & 0.00 & 0.00 & 0.10 & 0.25 & 0.18 \\
\hline Chelyabinsk oblast & 0.34 & 0.42 & 1.21 & 1.81 & 1.42 \\
\hline Chita oblast & 6.06 & 6.14 & 9.92 & 12.39 & 6.81 \\
\hline Chuvash Republic & 3.57 & 3.01 & 7.38 & 9.57 & 4.69 \\
\hline $\begin{array}{l}\text { Chukotka autono- } \\
\text { mous okrug }\end{array}$ & 39.93 & 34.58 & 28.29 & 25.37 & 34.98 \\
\hline $\begin{array}{l}\text { Evenk autonomous } \\
\text { okrug }\end{array}$ & 104.76 & 99.69 & 54.06 & 76.32 & 106.01 \\
\hline $\begin{array}{l}\text { Yamal-Nenetsky } \\
\text { autonomous okrug }\end{array}$ & 0.00 & 0.10 & 0.55 & 0.60 & 0.31 \\
\hline Yaroslavl oblast & 0.55 & 0.35 & 0.97 & 1.21 & 1.55 \\
\hline
\end{tabular}


Table A1-8

Financial Aid to Regions to Revenues to the Consolidated Budget of the Subject of the Federation over 1994-1998, as \%

\begin{tabular}{|c|c|c|c|c|c|}
\hline & 1994 & 1995 & 1996 & 1997 & 1998 \\
\hline 1 & 2 & 3 & 4 & 5 & 6 \\
\hline $\begin{array}{l}\text { Aginsky Buryatsky } \\
\text { autonomous okrug }\end{array}$ & 409.07 & 69.85 & 73.26 & 74.22 & 81.82 \\
\hline Altay krai & 84.62 & 32.79 & 42.53 & 44.03 & 42.43 \\
\hline Amur oblast & 53.30 & 29.66 & 48.30 & 47.27 & 42.62 \\
\hline Arkhangel' oblast & 31.34 & 22.61 & 21.17 & 14.13 & 14.54 \\
\hline Astarkhan oblast & 48.69 & 20.11 & 36.50 & 31.75 & 28.83 \\
\hline Belgorod oblast & 19.48 & 7.07 & 9.81 & 7.12 & 11.34 \\
\hline Bryansk oblast & 26.46 & 20.70 & 30.81 & 19.42 & 24.84 \\
\hline Vladimir oblast & 27.17 & 11.23 & 28.42 & 23.24 & 25.88 \\
\hline Volgograd oblast & 18.49 & 5.07 & 13.39 & 8.72 & 16.42 \\
\hline Vologda oblast & 15.94 & 8.11 & 17.99 & 13.57 & 3.37 \\
\hline Voronezh oblast & 18.41 & 11.89 & 19.57 & 17.36 & 19.44 \\
\hline City of Moscow & 19.13 & 6.70 & 6.52 & 4.53 & 1.32 \\
\hline Saint Petesrburg & 8.60 & 1.47 & 3.63 & 6.31 & 0.68 \\
\hline $\begin{array}{l}\text { Jewish Autonomous } \\
\text { oblast }\end{array}$ & 70.60 & 54.43 & 54.14 & 58.22 & 58.18 \\
\hline Ivanovo oblast & 37.16 & 33.94 & 45.94 & 45.55 & 30.63 \\
\hline Irkutsk oblast & 18.37 & 5.79 & 8.98 & 5.46 & 7.83 \\
\hline $\begin{array}{l}\text { Kabardino-Balkar Re- } \\
\text { public }\end{array}$ & 219.29 & 44.96 & 51.85 & 55.01 & 59.07 \\
\hline Kaliningrad oblast & 27.82 & 12.03 & 21.62 & 16.47 & 9.50 \\
\hline Kaluga oblast & 34.09 & 19.73 & 37.91 & 31.09 & 33.67 \\
\hline Kamchatka oblast & 99.23 & 32.21 & 30.70 & 47.49 & 42.11 \\
\hline $\begin{array}{l}\text { Karachay-Cherkess } \\
\text { Republic }\end{array}$ & 145.59 & 41.63 & 47.23 & 50.97 & 50.23 \\
\hline Kemerovo oblast & 35.47 & 14.90 & 25.91 & 15.65 & 18.33 \\
\hline Kirov oblast & 22.62 & 10.76 & 24.30 & 24.59 & 27.79 \\
\hline $\begin{array}{l}\text { Komi-Permyak autono- } \\
\text { mous okrug }\end{array}$ & 218.39 & 52.80 & 60.31 & 49.78 & 66.83 \\
\hline $\begin{array}{l}\text { Koryak autonomous } \\
\text { okrug }\end{array}$ & 642.52 & 81.79 & 71.62 & 55.48 & 60.67 \\
\hline Kostroma oblast & 55.29 & 46.88 & 60.17 & 29.30 & 28.92 \\
\hline Krasnodar krai & 24.44 & 10.47 & 18.81 & 24.08 & 17.36 \\
\hline Krasnoyarsky krai & 14.77 & 1.02 & 4.31 & 3.16 & 0.29 \\
\hline
\end{tabular}




\begin{tabular}{|c|c|c|c|c|c|}
\hline 1 & 2 & 3 & 4 & 5 & 6 \\
\hline Kurgan oblast & 49.02 & 13.71 & 29.53 & 26.12 & 32.85 \\
\hline Kursk oblast & 17.97 & 12.44 & 11.15 & 24.92 & 14.55 \\
\hline Leningrad oblast & 19.88 & 9.96 & 6.36 & 12.80 & 6.54 \\
\hline Lipetsk oblast & 15.64 & 2.06 & 1.18 & 1.52 & 1.89 \\
\hline Magadan oblast & 117.46 & 34.86 & 39.54 & 32.72 & 30.83 \\
\hline Moscow oblast & 17.22 & 8.73 & 9.96 & 10.27 & 2.90 \\
\hline Murmansk oblast & 24.10 & 17.63 & 29.24 & 32.74 & 28.51 \\
\hline $\begin{array}{l}\text { Nenetsky autonomous } \\
\text { okrug }\end{array}$ & 77.06 & 25.24 & 29.54 & 29.78 & 16.08 \\
\hline N. Novgorod oblast & 6.33 & 1.04 & 10.11 & 5.77 & 4.47 \\
\hline Novgorod oblast & 44.77 & 28.82 & 32.95 & 36.95 & 37.14 \\
\hline Novossibirsk oblast & 22.07 & 7.01 & 13.22 & 17.72 & 6.61 \\
\hline Omsk oblast & 33.79 & 12.84 & 21.01 & 9.15 & 11.69 \\
\hline Orenburg oblast & 29.94 & 8.14 & 12.02 & 10.04 & 22.02 \\
\hline Orel oblast & 56.68 & 34.03 & 39.26 & 39.69 & 21.93 \\
\hline Penza oblast & 61.41 & 20.23 & 34.59 & 24.51 & 26.15 \\
\hline Perm oblast & 13.99 & 6.09 & 10.10 & 7.90 & 2.02 \\
\hline Primorye krai & 36.98 & 23.36 & 23.08 & 34.02 & 31.48 \\
\hline Pskov oblast & 40.22 & 25.14 & 50.15 & 49.50 & 40.38 \\
\hline Republic Of Adygeya & 99.31 & 34.37 & 55.00 & 54.10 & 64.55 \\
\hline Altay Republic & 415.36 & 74.49 & 69.45 & 66.98 & 67.35 \\
\hline $\begin{array}{l}\text { Republic of Bashkor- } \\
\text { tostan }\end{array}$ & 3.60 & 0.00 & 0.04 & 0.00 & 4.71 \\
\hline Republic of Buryatia & 62.34 & 32.26 & 32.33 & 41.11 & 48.73 \\
\hline Republic of Dagestan & 690.26 & 81.63 & 71.55 & 73.79 & 80.23 \\
\hline Ingoush Republic & 1009.92 & 75.13 & 81.78 & 83.85 & 77.89 \\
\hline Kalmyk Republic & 346.01 & 69.95 & 67.52 & 54.82 & 62.29 \\
\hline Republic of Karelia & 17.03 & 16.01 & 21.20 & 18.66 & 14.42 \\
\hline Republic Komi & 31.97 & 14.13 & 18.31 & 7.87 & 4.59 \\
\hline Republic of Mary El & 71.82 & 31.80 & 37.82 & 37.47 & 27.36 \\
\hline Republic of Mordovia & 45.63 & 31.17 & 45.04 & 37.51 & 32.58 \\
\hline $\begin{array}{l}\text { Republic of Sakha } \\
\text { (Yakutia) }\end{array}$ & 2.32 & 1.07 & 36.83 & 36.82 & 17.13 \\
\hline $\begin{array}{l}\text { Republic of North } \\
\text { Ossetia (Alania) }\end{array}$ & 191.79 & 53.08 & 54.12 & 46.65 & 58.68 \\
\hline Republic of Tatarstan & 13.25 & 1.61 & 2.73 & 1.43 & 4.92 \\
\hline Republic of Tyva & 345.91 & 76.71 & 74.07 & 72.07 & 79.42 \\
\hline
\end{tabular}




\begin{tabular}{|c|c|c|c|c|c|}
\hline 1 & 2 & 3 & 4 & 5 & 6 \\
\hline Republic of Khakassia & 37.40 & 11.07 & 15.58 & 12.47 & 12.74 \\
\hline Rostov oblast & 17.55 & 10.10 & 30.29 & 21.79 & 20.62 \\
\hline Ryazan oblast & 29.19 & 3.97 & 22.94 & 22.68 & 17.38 \\
\hline Samara oblast & 10.15 & 1.59 & 1.71 & 2.15 & 3.85 \\
\hline Saratov oblast & 23.01 & 11.78 & 19.49 & 19.27 & 23.82 \\
\hline Sakhalin oblast & 44.20 & 30.02 & 33.51 & 32.97 & 25.78 \\
\hline Sverdlovsk oblast & 8.95 & 5.90 & 1.97 & 5.46 & 2.76 \\
\hline Smolensk oblast & 19.87 & 7.71 & 17.45 & 17.71 & 23.52 \\
\hline Stavropol krai & 52.85 & 20.85 & 30.54 & 28.90 & 27.48 \\
\hline $\begin{array}{l}\text { Taymyr (Dolgan- } \\
\text { Nenetsky) autonomous } \\
\text { okrug }\end{array}$ & 78.02 & 25.90 & 33.94 & 23.69 & 10.62 \\
\hline Tambov oblast & 35.08 & 19.23 & 23.54 & 24.92 & 29.17 \\
\hline Tver oblast & 25.58 & 13.56 & 26.11 & 27.53 & 14.96 \\
\hline Tomsk oblast & 45.07 & 11.75 & 14.09 & 14.36 & 12.22 \\
\hline Tula oblast & 25.79 & 8.53 & 30.90 & 27.68 & 15.46 \\
\hline Tyumen oblast & 35.93 & 7.14 & 12.35 & 12.49 & 11.56 \\
\hline Udmurt Republic & 33.09 & 10.45 & 24.66 & 26.44 & 23.78 \\
\hline Ulyanovsk oblast & 36.88 & 11.90 & 17.89 & 13.80 & 18.65 \\
\hline $\begin{array}{l}\text { Ust-Ordynsky Buryatsky } \\
\text { autonomous okrug }\end{array}$ & 299.57 & 67.96 & 70.10 & 64.70 & 71.96 \\
\hline Khabarovsk krai & 51.94 & 23.75 & 26.49 & 29.50 & 18.68 \\
\hline $\begin{array}{l}\text { Khanty-Mansy autono- } \\
\text { mous okrug }\end{array}$ & 3.55 & 1.65 & 3.84 & 0.04 & 1.37 \\
\hline Chelyabinsk oblast & 14.20 & 7.79 & 11.69 & 16.07 & 5.12 \\
\hline Chita oblast & 56.39 & 17.92 & 23.51 & 27.36 & 29.48 \\
\hline Chuvash Republic & 19.54 & 13.88 & 21.54 & 23.43 & 14.24 \\
\hline $\begin{array}{l}\text { Chukotka autonomous } \\
\text { okrug }\end{array}$ & 159.15 & 50.75 & 29.08 & 49.39 & 55.94 \\
\hline $\begin{array}{l}\text { Evenk autonomous } \\
\text { okrug }\end{array}$ & 165.14 & 61.13 & 43.00 & 60.12 & 47.79 \\
\hline $\begin{array}{l}\text { Yamal-Nenetsky } \\
\text { autonomous okrug }\end{array}$ & 4.17 & 1.46 & 2.03 & 0.08 & 0.17 \\
\hline Yaroslavl oblast & 10.94 & 3.68 & 20.74 & 18.73 & 8.48 \\
\hline
\end{tabular}


Table A1-9

Financial Aid to Regions to Revenues to the Consolidated Budget of the Subject of the Federation over 1999-2002, as \%

\begin{tabular}{|c|c|c|c|c|c|}
\hline & 1999 & 2000 & 2001 & 2002 & $\begin{array}{c}\text { Average } \\
\text { over 1994- } \\
2002 \\
\end{array}$ \\
\hline 1 & 2 & 3 & 4 & 5 & 6 \\
\hline $\begin{array}{l}\text { Aginsky Buryatsky } \\
\text { autonomous okrug }\end{array}$ & 52.16 & 84.17 & 77.32 & 58.92 & 108.98 \\
\hline Altay krai & 42.55 & 46.81 & 56.58 & 60.28 & 50.29 \\
\hline Amur oblast & 37.70 & 44.51 & 48.75 & 45.24 & 44.15 \\
\hline Arkhangel' oblast & 18.56 & 22.13 & 34.11 & 35.24 & 23.76 \\
\hline Astarkhan oblast & 20.98 & 16.39 & 14.99 & 18.71 & 26.33 \\
\hline Belgorod oblast & 3.12 & 6.80 & 11.39 & 16.19 & 10.26 \\
\hline Bryansk oblast & 27.36 & 45.82 & 50.11 & 41.90 & 31.93 \\
\hline Vladimir oblast & 16.49 & 23.44 & 28.04 & 28.44 & 23.60 \\
\hline Volgograd oblast & 11.10 & 5.52 & 16.22 & 14.83 & 12.20 \\
\hline Vologda oblast & 2.76 & 0.34 & 9.40 & 11.76 & 9.25 \\
\hline Voronezh oblast & 15.95 & 18.24 & 31.75 & 29.81 & 20.27 \\
\hline City of Moscow & 0.00 & 0.00 & 3.02 & -19.42 & 2.42 \\
\hline Saint Petesrburg & 0.50 & 0.38 & 4.58 & 7.91 & 3.79 \\
\hline Jewish Autonomous oblast & 61.12 & 64.52 & 74.18 & 66.16 & 62.39 \\
\hline Ivanovo oblast & 35.52 & 47.14 & 46.89 & 50.54 & 41.48 \\
\hline Irkutsk oblast & 7.89 & 6.18 & 12.86 & 11.88 & 9.47 \\
\hline Kabardino-Balkar Republic & 57.81 & 49.06 & 56.28 & 69.39 & 73.64 \\
\hline Kaliningrad oblast & 9.85 & 12.05 & 20.67 & 23.20 & 17.02 \\
\hline Kaluga oblast & 19.05 & 22.63 & 34.56 & 36.84 & 29.95 \\
\hline Kamchatka oblast & 45.60 & 56.40 & 45.81 & 48.51 & 49.79 \\
\hline $\begin{array}{l}\text { Karachay-Cherkess Repub- } \\
\text { lic }\end{array}$ & 55.43 & 62.23 & 65.60 & 77.62 & 66.28 \\
\hline Kemerovo oblast & 11.83 & 12.90 & 21.44 & 19.08 & 19.50 \\
\hline Kirov oblast & 15.83 & 12.63 & 31.46 & 31.45 & 22.38 \\
\hline $\begin{array}{l}\text { Komi-Permyak autonomous } \\
\text { okrug }\end{array}$ & 66.25 & 75.32 & 77.75 & 79.70 & 83.02 \\
\hline Koryak autonomous okrug & 63.57 & 64.72 & 67.16 & 71.83 & 131.04 \\
\hline Kostroma oblast & 23.58 & 26.10 & 31.94 & 40.05 & 38.03 \\
\hline Krasnodar krai & 10.09 & 11.70 & 18.66 & 27.46 & 18.12 \\
\hline Krasnoyarsky krai & 3.73 & 0.54 & 6.03 & 6.46 & 4.48 \\
\hline
\end{tabular}




\begin{tabular}{|c|c|c|c|c|c|}
\hline 1 & 2 & 3 & 4 & 5 & 6 \\
\hline Kurgan oblast & 25.62 & 23.68 & 44.36 & 53.36 & 33.14 \\
\hline Kursk oblast & 10.52 & 13.95 & 21.78 & 27.21 & 17.17 \\
\hline Leningrad oblast & 4.13 & 2.29 & 11.79 & 11.46 & 9.47 \\
\hline Lipetsk oblast & 1.96 & 0.56 & 6.14 & 5.73 & 4.08 \\
\hline Magadan oblast & 36.54 & 38.49 & 46.58 & 49.43 & 47.38 \\
\hline Moscow oblast & 3.03 & 1.13 & 6.38 & 7.48 & 7.46 \\
\hline Murmansk oblast & 17.66 & 27.67 & 11.80 & 13.87 & 22.58 \\
\hline $\begin{array}{l}\text { Nenetsky autonomous } \\
\text { okrug }\end{array}$ & 19.87 & 7.07 & 8.48 & 5.41 & 24.28 \\
\hline N. Novgorod oblast & 3.08 & 3.42 & 9.45 & 11.27 & 6.10 \\
\hline Novgorod oblast & 16.34 & 29.12 & 20.53 & 23.55 & 30.02 \\
\hline Novossibirsk oblast & 8.89 & 13.12 & 20.80 & 23.06 & 14.72 \\
\hline Omsk oblast & 12.49 & 18.05 & 26.38 & 30.65 & 19.56 \\
\hline Orenburg oblast & 6.22 & 5.40 & 8.83 & 13.10 & 12.86 \\
\hline Orel oblast & 17.03 & 29.02 & 22.81 & 32.74 & 32.58 \\
\hline Penza oblast & 28.06 & 34.51 & 45.80 & 50.83 & 36.23 \\
\hline Perm oblast & 0.41 & 1.68 & 4.49 & 5.68 & 5.82 \\
\hline Primorye krai & 26.98 & 31.38 & 40.74 & 35.64 & 31.52 \\
\hline Pskov oblast & 33.13 & 44.98 & 44.38 & 49.96 & 41.98 \\
\hline Republic Of Adygeya & 56.91 & 49.10 & 57.19 & 68.45 & 59.89 \\
\hline Altay Republic & 54.45 & 61.63 & 60.33 & 62.37 & 103.60 \\
\hline Republic of Bashkortostan & 0.24 & 7.12 & 25.38 & 23.15 & 7.14 \\
\hline Republic of Buryatia & 44.64 & 44.61 & 47.14 & 55.46 & 45.40 \\
\hline Republic of Dagestan & 77.42 & 83.07 & 82.05 & 79.95 & 146.66 \\
\hline Ingoush Republic & 69.93 & 45.79 & 79.08 & 89.83 & 179.24 \\
\hline Kalmyk Republic & 46.13 & 25.55 & 48.90 & 59.54 & 86.75 \\
\hline Republic of Karelia & 11.07 & 10.33 & 19.83 & 27.85 & 17.38 \\
\hline Republic Komi & 2.15 & 0.99 & 4.93 & 9.08 & 10.45 \\
\hline Republic of Mary El & 35.02 & 35.18 & 48.09 & 53.98 & 42.06 \\
\hline Republic of Mordovia & 34.16 & 25.70 & 34.24 & 38.58 & 36.07 \\
\hline $\begin{array}{l}\text { Republic of Sakha (Ya- } \\
\text { kutia) }\end{array}$ & 19.52 & 13.72 & 27.82 & 28.77 & 20.45 \\
\hline $\begin{array}{l}\text { Republic of North Ossetia } \\
\text { (Alania) }\end{array}$ & 50.72 & 43.26 & 55.61 & 64.17 & 68.68 \\
\hline Republic of Tatarstan & 0.72 & 0.70 & 26.11 & 25.66 & 8.57 \\
\hline Republic of Tyva & 81.00 & 85.41 & 86.60 & 84.33 & 109.50 \\
\hline
\end{tabular}




\begin{tabular}{|c|c|c|c|c|c|}
\hline 1 & 2 & 3 & 4 & 5 & 6 \\
\hline Republic of Khakassia & 15.25 & 13.09 & 23.54 & 22.61 & 18.19 \\
\hline Rostov oblast & 17.52 & 19.68 & 26.44 & 32.21 & 21.80 \\
\hline Ryazan oblast & 15.50 & 15.84 & 28.15 & 18.08 & 19.31 \\
\hline Samara oblast & 0.19 & 0.54 & 3.93 & 5.23 & 3.26 \\
\hline Saratov oblast & 14.21 & 14.08 & 21.18 & 23.72 & 18.95 \\
\hline Sakhalin oblast & 33.35 & 34.12 & 32.97 & 31.22 & 33.13 \\
\hline Sverdlovsk oblast & 3.63 & 1.54 & 6.27 & 6.37 & 4.76 \\
\hline Smolensk oblast & 11.72 & 27.39 & 18.64 & 21.93 & 18.44 \\
\hline Stavropol krai & 18.02 & 26.78 & 30.52 & 40.10 & 30.67 \\
\hline $\begin{array}{l}\text { Taymyr (Dolgan-Nenetsky) } \\
\text { autonomous okrug }\end{array}$ & 26.46 & 13.01 & 24.97 & 35.10 & 30.19 \\
\hline Tambov oblast & 24.23 & 31.18 & 44.14 & 45.74 & 30.80 \\
\hline Tver oblast & 15.26 & 32.27 & 30.34 & 25.78 & 23.49 \\
\hline Tomsk oblast & 8.88 & 10.16 & 12.40 & 19.77 & 16.52 \\
\hline Tula oblast & 10.95 & 17.05 & 22.13 & 20.61 & 19.90 \\
\hline Tyumen oblast & 5.88 & 0.33 & 3.82 & 4.73 & 10.47 \\
\hline Udmurt Republic & 11.40 & 7.01 & 12.04 & 18.30 & 18.57 \\
\hline Ulyanovsk oblast & 10.00 & 8.56 & 29.12 & 32.94 & 19.97 \\
\hline $\begin{array}{l}\text { Ust-Ordynsky Buryatsky } \\
\text { autonomous okrug }\end{array}$ & 76.36 & 81.42 & 82.82 & 84.08 & 99.88 \\
\hline Khabarovsk krai & 22.56 & 19.62 & 23.43 & 23.50 & 26.61 \\
\hline $\begin{array}{l}\text { Khanty-Mansy autonomous } \\
\text { okrug }\end{array}$ & 0.00 & 0.00 & 0.64 & 1.78 & 1.43 \\
\hline Chelyabinsk oblast & 2.53 & 3.55 & 9.54 & 13.51 & 9.33 \\
\hline Chita oblast & 41.45 & 41.03 & 45.36 & 47.69 & 36.69 \\
\hline Chuvash Republic & 21.24 & 18.91 & 39.45 & 42.34 & 23.84 \\
\hline $\begin{array}{l}\text { Chukotka autonomous } \\
\text { okrug }\end{array}$ & 64.31 & 68.04 & 51.80 & 40.04 & 63.17 \\
\hline Evenk autonomous okrug & 79.13 & 87.30 & 50.76 & 30.64 & 69.45 \\
\hline $\begin{array}{l}\text { Yamal-Nenetsky autono- } \\
\text { mous okrug }\end{array}$ & 0.01 & 0.43 & 2.91 & 3.88 & 1.68 \\
\hline Yaroslavl oblast & 3.46 & 2.36 & 7.35 & 7.66 & 9.27 \\
\hline
\end{tabular}


Table A1-10

Investment in Capital Assets Funded at the Expense of Budget Resources to GRP over 1995-1998, as \%

\begin{tabular}{|c|c|c|c|c|}
\hline & 1995 & 1996 & 1997 & 1998 \\
\hline 1 & 2 & 3 & 4 & 5 \\
\hline $\begin{array}{l}\text { Aginsky Buryatsky autono- } \\
\text { mous okrug }\end{array}$ & 0.00 & 0.00 & 0.00 & 2.85 \\
\hline Altay krai & 0.00 & 0.00 & 0.00 & 3.68 \\
\hline Amur oblast & 0.00 & 0.00 & 0.00 & 4.47 \\
\hline Arkhangel' oblast & 2.14 & 2.17 & 1.58 & 0.93 \\
\hline Astarkhan oblast & 3.20 & 2.37 & 2.41 & 2.71 \\
\hline Belgorod oblast & 4.02 & 3.61 & 4.56 & 3.86 \\
\hline Bryansk oblast & 6.30 & 3.66 & 2.53 & 1.97 \\
\hline Vladimir oblast & 1.39 & 1.40 & 1.14 & 1.94 \\
\hline Volgograd oblast & 2.80 & 2.70 & 3.17 & 2.25 \\
\hline Vologda oblast & 1.74 & 1.73 & 1.98 & 1.74 \\
\hline Voronezh oblast & 2.11 & 3.36 & 2.38 & 2.69 \\
\hline City of Moscow & 8.05 & 7.36 & 6.53 & 4.12 \\
\hline Saint Petesrburg & 3.57 & 3.48 & 2.59 & 2.02 \\
\hline Jewish Autonomous oblast & 0.00 & 0.00 & 0.00 & 12.09 \\
\hline Ivanovo oblast & 1.68 & 2.30 & 2.01 & 7.02 \\
\hline Irkutsk oblast & 0.00 & 0.00 & 0.00 & 2.08 \\
\hline Kabardino-Balkar Republic & 6.50 & 8.73 & 9.20 & 10.31 \\
\hline Kaliningrad oblast & 0.00 & 0.00 & 0.00 & 1.66 \\
\hline Kaluga oblast & 3.08 & 2.78 & 3.76 & 2.81 \\
\hline Kamchatka oblast & 0.00 & 0.00 & 0.00 & 2.01 \\
\hline Karachay-Cherkess Republic & 1.63 & 2.96 & 4.60 & 6.41 \\
\hline Kemerovo oblast & 0.00 & 0.00 & 0.00 & 3.10 \\
\hline Kirov oblast & 1.50 & 1.86 & 1.65 & 1.58 \\
\hline $\begin{array}{l}\text { Komi-Permyak autonomous } \\
\text { okrug }\end{array}$ & 2.09 & 1.42 & 0.91 & 2.78 \\
\hline Koryak autonomous okrug & 0.00 & 0.00 & 0.00 & 0.31 \\
\hline Kostroma oblast & 2.71 & 1.58 & 2.28 & 1.78 \\
\hline Krasnodar krai & 1.96 & 1.91 & 1.66 & 2.64 \\
\hline Krasnoyarsky krai & 0.00 & 0.00 & 0.00 & 1.87 \\
\hline Kurgan oblast & 2.88 & 3.05 & 3.06 & 1.77 \\
\hline
\end{tabular}




\begin{tabular}{|c|c|c|c|c|}
\hline 1 & 2 & 3 & 4 & 5 \\
\hline Kursk oblast & 2.12 & 2.08 & 2.67 & 1.69 \\
\hline Leningrad oblast & 1.83 & 1.99 & 2.93 & 3.88 \\
\hline Lipetsk oblast & 2.14 & 4.17 & 4.44 & 4.86 \\
\hline Magadan oblast & 0.00 & 0.00 & 0.00 & 2.98 \\
\hline Moscow oblast & 5.46 & 3.77 & 4.00 & 3.23 \\
\hline Murmansk oblast & 2.33 & 1.88 & 1.47 & 1.38 \\
\hline Nenetsky autonomous okrug & 1.94 & 2.89 & 3.08 & 4.72 \\
\hline N. Novgorod oblast & 2.48 & 2.69 & 3.14 & 2.05 \\
\hline Novgorod oblast & 2.10 & 1.15 & 2.54 & 0.70 \\
\hline Novossibirsk oblast & 0.00 & 0.00 & 0.00 & 2.83 \\
\hline Omsk oblast & 0.00 & 0.00 & 0.00 & 3.22 \\
\hline Orenburg oblast & 2.27 & 2.22 & 2.61 & 2.25 \\
\hline Orel oblast & 2.34 & 6.32 & 4.75 & 6.12 \\
\hline Penza oblast & 3.03 & 2.58 & 2.81 & 5.88 \\
\hline Perm oblast & 1.07 & 1.55 & 2.17 & 1.23 \\
\hline Primorye krai & 0.00 & 0.00 & 0.00 & 2.56 \\
\hline Pskov oblast & 3.36 & 3.83 & 2.69 & 3.83 \\
\hline Republic Of Adygeya & 3.77 & 5.53 & 3.36 & 3.28 \\
\hline Altay Republic & 0.00 & 0.00 & 0.00 & 3.64 \\
\hline Republic of Bashkortostan & 4.05 & 3.26 & 2.44 & 3.56 \\
\hline Republic of Buryatia & 0.00 & 0.00 & 0.00 & 3.36 \\
\hline Republic of Dagestan & 12.55 & 10.29 & 8.32 & 11.32 \\
\hline Ingoush Republic & 56.14 & 36.80 & 50.29 & 36.00 \\
\hline Kalmyk Republic & 8.27 & 9.17 & 10.92 & 22.08 \\
\hline Republic of Karelia & 1.81 & 2.35 & 2.80 & 1.77 \\
\hline Republic Komi & 2.46 & 6.37 & 4.30 & 1.04 \\
\hline Republic of Mary El & 3.03 & 3.68 & 3.14 & 3.87 \\
\hline Republic of Mordovia & 2.01 & 4.38 & 4.40 & 4.62 \\
\hline Republic of Sakha (Yakutia) & 0.00 & 0.00 & 0.00 & 3.20 \\
\hline $\begin{array}{l}\text { Republic of North Ossetia } \\
\text { (Alania) }\end{array}$ & 7.98 & 10.34 & 9.27 & 8.36 \\
\hline Republic of Tatarstan & 3.26 & 3.43 & 3.06 & 1.20 \\
\hline Republic of Tyva & 0.00 & 0.00 & 0.00 & 8.48 \\
\hline Republic of Khakassia & 0.00 & 0.00 & 0.00 & 3.09 \\
\hline Rostov oblast & 2.46 & 3.74 & 3.34 & 3.10 \\
\hline
\end{tabular}




\begin{tabular}{|c|c|c|c|c|}
\hline 1 & 2 & 3 & 4 & 5 \\
\hline Ryazan oblast & 1.81 & 1.59 & 2.72 & 1.43 \\
\hline Samara oblast & 1.94 & 1.51 & 1.38 & 1.05 \\
\hline Saratov oblast & 1.68 & 4.63 & 6.52 & 5.83 \\
\hline Sakhalin oblast & 0.00 & 0.00 & 0.00 & 3.23 \\
\hline Sverdlovsk oblast & 2.37 & 2.80 & 3.52 & 2.70 \\
\hline Smolensk oblast & 1.78 & 1.44 & 2.09 & 1.30 \\
\hline Stavropol krai & 4.85 & 3.38 & 3.34 & 3.90 \\
\hline $\begin{array}{l}\text { Taymyr (Dolgan-Nenetsky) } \\
\text { autonomous okrug }\end{array}$ & 0.00 & 0.00 & 0.00 & 3.44 \\
\hline Tambov oblast & 2.83 & 3.01 & 2.51 & 1.98 \\
\hline Tver oblast & 2.22 & 3.58 & 2.56 & 2.34 \\
\hline Tomsk oblast & 0.00 & 0.00 & 0.00 & 2.35 \\
\hline Tula oblast & 2.99 & 5.15 & 2.80 & 2.98 \\
\hline Tyumen oblast & 0.00 & 0.00 & 0.00 & 0.39 \\
\hline Udmurt Republic & 3.03 & 3.02 & 2.09 & 1.91 \\
\hline Ulyanovsk oblast & 2.19 & 1.74 & 2.03 & 4.61 \\
\hline $\begin{array}{l}\text { Ust-Ordynsky Buryatsky } \\
\text { autonomous okrug }\end{array}$ & 0.00 & 0.00 & 0.00 & 4.70 \\
\hline Khabarovsk krai & 0.00 & 0.00 & 0.00 & 2.95 \\
\hline $\begin{array}{l}\text { Khanty-Mansy autonomous } \\
\text { okrug }\end{array}$ & 0.00 & 0.00 & 0.00 & 2.96 \\
\hline Chelyabinsk oblast & 2.91 & 4.41 & 4.52 & 3.81 \\
\hline Chita oblast & 0.00 & 0.00 & 0.00 & 2.39 \\
\hline Chuvash Republic & 4.31 & 3.90 & 5.15 & 5.93 \\
\hline Chukotka autonomous okrug & 0.00 & 0.00 & 0.00 & 5.02 \\
\hline Evenk autonomous okrug & 0.00 & 0.00 & 0.00 & 11.51 \\
\hline $\begin{array}{l}\text { Yamal-Nenetsky autonomous } \\
\text { okrug }\end{array}$ & 0.00 & 0.00 & 0.00 & 7.19 \\
\hline Yaroslavl oblast & 2.23 & 1.22 & 1.75 & 2.12 \\
\hline
\end{tabular}


Table A1-11

Investment in Capital Assets Funded at the Expense of Budget Resources to

GRP over 1999-2002, as \%

\begin{tabular}{|c|c|c|c|c|c|}
\hline & 1999 & 2000 & 2001 & 2002 & inv1 \\
\hline 1 & 2 & 3 & 4 & 5 & 6 \\
\hline $\begin{array}{l}\text { Aginsky Buryatsky } \\
\text { autonomous okrug }\end{array}$ & 5.93 & 7.95 & 9.63 & 11.97 & 4.79 \\
\hline Altay krai & 3.73 & 4.57 & 2.65 & 2.53 & 2.14 \\
\hline Amur oblast & 3.76 & 4.01 & 4.49 & 7.18 & 2.99 \\
\hline Arkhangel' oblast & 0.90 & 0.85 & 1.41 & 0.51 & 1.31 \\
\hline Astarkhan oblast & 1.98 & 2.68 & 2.61 & 3.20 & 2.65 \\
\hline Belgorod oblast & 2.84 & 2.93 & 2.12 & 2.24 & 3.27 \\
\hline Bryansk oblast & 1.78 & 2.24 & 2.13 & 2.28 & 2.86 \\
\hline Vladimir oblast & 1.40 & 2.92 & 2.47 & 2.24 & 1.86 \\
\hline Volgograd oblast & 1.77 & 2.19 & 1.26 & 1.32 & 2.18 \\
\hline Vologda oblast & 1.69 & 2.03 & 1.82 & 1.34 & 1.76 \\
\hline Voronezh oblast & 2.63 & 3.29 & 2.44 & 2.35 & 2.66 \\
\hline City of Moscow & 2.13 & 5.98 & 4.49 & 4.48 & 5.39 \\
\hline Saint Petesrburg & 3.62 & 3.20 & 5.50 & 3.85 & 3.48 \\
\hline $\begin{array}{l}\text { Jewish Autonomous } \\
\text { oblast }\end{array}$ & 8.41 & 3.96 & 3.22 & 5.01 & 4.09 \\
\hline Ivanovo oblast & 1.08 & 1.53 & 3.68 & 3.61 & 2.86 \\
\hline Irkutsk oblast & 2.39 & 2.73 & 1.81 & 1.60 & 1.33 \\
\hline $\begin{array}{l}\text { Kabardino-Balkar Re- } \\
\text { public }\end{array}$ & 6.21 & 9.13 & 7.28 & 7.83 & 8.15 \\
\hline Kaliningrad oblast & 1.91 & 2.88 & 2.84 & 2.65 & 1.49 \\
\hline Kaluga oblast & 3.54 & 5.12 & 4.02 & 3.15 & 3.53 \\
\hline Kamchatka oblast & 1.46 & 4.98 & 5.14 & 4.64 & 2.28 \\
\hline $\begin{array}{l}\text { Karachay-Cherkess } \\
\text { Republic }\end{array}$ & 3.90 & 5.16 & 6.24 & 6.14 & 4.63 \\
\hline Kemerovo oblast & 2.88 & 3.67 & 2.20 & 1.58 & 1.68 \\
\hline Kirov oblast & 1.97 & 1.96 & 1.98 & 1.89 & 1.80 \\
\hline $\begin{array}{l}\text { Komi-Permyak autono- } \\
\text { mous okrug }\end{array}$ & 1.11 & 2.26 & 5.68 & 11.13 & 3.42 \\
\hline $\begin{array}{l}\text { Koryak autonomous } \\
\text { okrug }\end{array}$ & 0.65 & 2.37 & 4.89 & 8.96 & 2.15 \\
\hline Kostroma oblast & 1.95 & 2.95 & 4.40 & 3.27 & 2.62 \\
\hline Krasnodar krai & 2.43 & 3.72 & 2.27 & 2.32 & 2.36 \\
\hline Krasnoyarsky krai & 1.30 & 1.02 & 2.06 & 1.33 & 0.95 \\
\hline
\end{tabular}

86 


\begin{tabular}{|c|c|c|c|c|c|}
\hline 1 & 2 & 3 & 4 & 5 & 6 \\
\hline Kurgan oblast & 2.38 & 2.63 & 1.92 & 2.46 & 2.52 \\
\hline Kursk oblast & 1.98 & 2.36 & 2.21 & 2.05 & 2.14 \\
\hline Leningrad oblast & 3.48 & 3.83 & 3.21 & 3.15 & 3.04 \\
\hline Lipetsk oblast & 2.47 & 2.73 & 1.81 & 0.90 & 2.94 \\
\hline Magadan oblast & 3.49 & 4.70 & 3.81 & 3.71 & 2.33 \\
\hline Moscow oblast & 6.13 & 4.84 & 3.81 & 2.01 & 4.15 \\
\hline Murmansk oblast & 1.16 & 1.09 & 1.47 & 2.05 & 1.60 \\
\hline $\begin{array}{l}\text { Nenetsky autonomous } \\
\text { okrug }\end{array}$ & 1.95 & 2.47 & 3.05 & 2.45 & 2.82 \\
\hline N. Novgorod oblast & 1.77 & 2.50 & 1.58 & 1.85 & 2.26 \\
\hline Novgorod oblast & 1.25 & 1.38 & 0.86 & 0.85 & 1.35 \\
\hline Novossibirsk oblast & 1.78 & 2.90 & 2.60 & 2.47 & 1.57 \\
\hline Omsk oblast & 2.11 & 1.98 & 1.96 & 1.63 & 1.36 \\
\hline Orenburg oblast & 1.16 & 1.13 & 2.45 & 1.39 & 1.93 \\
\hline Orel oblast & 5.02 & 2.80 & 3.17 & 1.48 & 4.00 \\
\hline Penza oblast & 3.48 & 4.20 & 6.24 & 4.69 & 4.12 \\
\hline Perm oblast & 1.27 & 1.64 & 1.58 & 2.49 & 1.63 \\
\hline Primorye krai & 1.77 & 1.84 & 1.86 & 2.01 & 1.26 \\
\hline Pskov oblast & 3.97 & 5.77 & 5.03 & 3.61 & 4.01 \\
\hline Republic Of Adygeya & 4.06 & 4.75 & 12.16 & 10.02 & 5.87 \\
\hline Altay Republic & 2.46 & 3.43 & 14.57 & 13.55 & 4.70 \\
\hline $\begin{array}{l}\text { Republic of Bashkor- } \\
\text { tostan }\end{array}$ & 3.43 & 6.90 & 7.09 & 6.26 & 4.63 \\
\hline Republic of Buryatia & 1.79 & 3.05 & 3.14 & 3.46 & 1.85 \\
\hline Republic of Dagestan & 7.36 & 8.76 & 10.72 & 7.53 & 9.61 \\
\hline Ingoush Republic & 29.23 & 8.73 & 20.97 & 15.04 & 31.65 \\
\hline Kalmyk Republic & 13.38 & 3.15 & 3.24 & 4.23 & 9.31 \\
\hline Republic of Karelia & 2.36 & 3.57 & 3.37 & 3.04 & 2.63 \\
\hline Republic Komi & 1.19 & 1.85 & 0.00 & 1.27 & 2.31 \\
\hline Republic of Mary El & 4.15 & 4.81 & 3.64 & 3.76 & 3.76 \\
\hline Republic of Mordovia & 4.66 & 3.98 & 8.78 & 8.90 & 5.22 \\
\hline $\begin{array}{l}\text { Republic of Sakha } \\
\text { (Yakutia) }\end{array}$ & 4.69 & 5.74 & 5.18 & 3.37 & 2.77 \\
\hline $\begin{array}{l}\text { Republic of North } \\
\text { Ossetia (Alania) }\end{array}$ & 7.53 & 11.32 & 9.40 & 8.87 & 9.13 \\
\hline Republic of Tatarstan & 1.01 & 3.41 & 5.40 & 2.74 & 2.94 \\
\hline Republic of Tyva & 7.56 & 5.94 & 11.17 & 8.59 & 5.22 \\
\hline
\end{tabular}




\begin{tabular}{|c|c|c|c|c|c|}
\hline 1 & 2 & 3 & 4 & 5 & 6 \\
\hline Republic of Khakassia & 1.76 & 2.09 & 1.56 & 1.41 & 1.24 \\
\hline Rostov oblast & 2.81 & 3.65 & 3.46 & 3.26 & 3.23 \\
\hline Ryazan oblast & 1.25 & 1.98 & 1.23 & 2.59 & 1.83 \\
\hline Samara oblast & 0.78 & 0.91 & 2.74 & 1.74 & 1.51 \\
\hline Saratov oblast & 3.14 & 1.58 & 1.86 & 3.28 & 3.57 \\
\hline Sakhalin oblast & 3.05 & 3.76 & 2.37 & 1.76 & 1.77 \\
\hline Sverdlovsk oblast & 1.20 & 3.09 & 2.45 & 2.54 & 2.58 \\
\hline Smolensk oblast & 1.05 & 1.26 & 1.48 & 1.90 & 1.54 \\
\hline Stavropol krai & 3.97 & 3.64 & 2.92 & 2.26 & 3.53 \\
\hline $\begin{array}{l}\text { Taymyr (Dolgan- } \\
\text { Nenetsky) autonomous } \\
\text { okrug }\end{array}$ & 3.78 & 4.32 & 7.99 & 5.07 & 3.08 \\
\hline Tambov oblast & 1.67 & 1.81 & 1.96 & 2.61 & 2.30 \\
\hline Tver oblast & 3.47 & 3.33 & 2.49 & 2.52 & 2.81 \\
\hline Tomsk oblast & 1.82 & 0.97 & 1.78 & 1.03 & 0.99 \\
\hline Tula oblast & 3.54 & 10.87 & 4.45 & 2.68 & 4.43 \\
\hline Tyumen oblast & 0.27 & 2.39 & 2.21 & 0.17 & 0.68 \\
\hline Udmurt Republic & 1.91 & 2.36 & 2.63 & 2.01 & 2.37 \\
\hline Ulyanovsk oblast & 3.68 & 4.33 & 3.59 & 2.83 & 3.12 \\
\hline $\begin{array}{l}\text { Ust-rdynsky Buryatsky } \\
\text { autonomous okrug }\end{array}$ & 3.00 & 5.48 & 5.64 & 5.88 & 3.09 \\
\hline Khabarovsk krai & 3.09 & 2.14 & 4.72 & 3.51 & 2.05 \\
\hline $\begin{array}{l}\text { Khanty-Mansy autono- } \\
\text { mous okrug }\end{array}$ & 3.04 & 3.10 & 4.13 & 2.79 & 2.00 \\
\hline Chelyabinsk oblast & 3.26 & 4.28 & 3.80 & 2.88 & 3.73 \\
\hline Chita oblast & 1.99 & 1.82 & 2.82 & 4.23 & 1.66 \\
\hline Chuvash Republic & 4.98 & 6.91 & 6.25 & 6.02 & 5.43 \\
\hline $\begin{array}{l}\text { Chukotka autonomous } \\
\text { okrug }\end{array}$ & 4.72 & 5.41 & 8.97 & 7.70 & 3.98 \\
\hline $\begin{array}{l}\text { Evenk autonomous } \\
\text { okrug }\end{array}$ & 11.73 & 5.87 & 11.30 & 26.97 & 8.42 \\
\hline $\begin{array}{l}\text { Yamal-Nenetsky } \\
\text { autonomous okrug }\end{array}$ & 6.88 & 5.02 & 1.98 & 2.78 & 2.98 \\
\hline Yaroslavl oblast & 2.77 & 2.09 & 1.39 & 1.45 & 1.88 \\
\hline
\end{tabular}




\section{Decomposition of Economic Growth in Russia's Regions}

As shown in the previous chapter, Russian regions have demonstrated a great deal of diversity in terms of pace of their development. More specifically, it can be argued that, overall, those regions that at the starting moment had been poorer than others demonstrated a greater pace of growth over the whole period of observations, however the differentiation between the "extreme" regions continued to increase.

Decomposition of growth by factors can form the first step towards explanation of the inter-regional differentiation of economic growth rates. This particular procedure also forms the first step to identification of driving forces behind economic growth. The main purpose of the decomposition is to identify sources of growth and its division into extensive and intensive components. Intensive component is usually singled out in the form of an assessment of total factor productivity (TFP) or technical progress embodied in factors of growth (labor and capital).

In earlier IET's papers (Entov, Lugovoy at al. (2003)), their authors conducted decomposition of economic growth by factors on the sectoral and general economy levels. The research demonstrated that during the transition period a considerable rate of the dynamics of output in the industrial sector was determined by the loading rate of then existing capacities and intensity of the use of labor (see Table A2-1 and Fig. A2-1 in the Annex2). Meanwhile, the contribution rate of TFP to the growth of output in the industrial sector accounted for 20-50\%. At the level of GDP, economic growth unexplained by the basic factors (labor and capital) accounted for a far greater value - some 30-70\% (see Table A2-2 and Fig. A2-2 in the Annex 2). This particular level of unexplained eco- 
nomic growth is typical of the earliest computations of decomposition of growth (see, for example, Solow (1957), Kuznets (1996)), while consequent research demonstrated that the value of the remainder can be diminished considerably or even reduced to zero by means of a more accurate measure of growth factors ${ }^{25}$.

It should be noted that a greater accuracy of measuring requires a greater data detalization (disaggregation), which is not always possible, given the state of the national statistics in Russia. However, in the circumstances even fairly rough assessments may prove to be useful from the perspective of understanding of the ongoing processes and political decision making.

The earlier IET's research demonstrated that the dynamics of TFP advanced those of output. The productivity series had experienced a turn of the trend from the transformational decline to growth in 1-3 years earlier than output did.

The present paper constitutes an attempt to decompose economic growth of Subjects of the Russian Federation basing on the officially published statistical information.

\subsection{Methodology and Data}

The problem of decomposition of growth by factors, alias growth accounting, found itself among the most debated issues of the second half of the $20^{\text {th }}$ century. Despite the seemingly simple

\footnotetext{
${ }^{25}$ In their paper, Griliches and Jorgenson (1967) were the first to put forward a hypothesis on the possibility for total factor productivity being reduced to zero by means of a more accurate measuring of the factors. However the hypotheses suggests that technical progress is embodied in capital and labor. The discussion with Dennison allowed a demonstration of the fact that even under a more accurate measure of indicators of growth of output and factors the unexplained remainder remains substantial, anyway, albeit accounting for a considerably smaller value.
} 
task setting - that is, identification of a contribution of factors (labor, capital, among others) to the growth in output and thus singling out an unexplained by the factors remainder (usually interpreted as TFP), the main discussion centered on the problem of measuring the factors.

However, the research community has recently succeeded in mobilizing consensus on a number of key issues, albeit many of them still remain open for discussion. The main approaches and issues of decomposition still under discussion have been highlighted to a greatest possible extent in two OECD methodological papers (2001a and 2001b) and a NBER monography (2004). The aforementioned IET paper on analysis of factors of Russian economic growth (Entov, Lugovoy at al. (2003)) provides a review of main issues of decomposition of growth.

Given the narrowness of the available statistical base, the present paper considers the possibility for employment of various methods of decomposition (direct and dual) and measuring the employed indicators (output, labor and capital).

All the computations were conducted using the official information on socio-economic state of Subjects of the Russian Federation published by Rosstat. Naturally, not all the information needed for the purpose of the analysis has become available - the problem was remedied by introduction of various estimates or affected the employed methodology and interpretation of results. All the methodology and work with the data, as well as interpretation of the findings are given below.

\subsection{The Main Approach to Decomposition of Growth}

Underlying the method of decomposition of growth is the assumption of the existence of a macroeconomic production function that determines a correlation between the maximum possible vol- 
ume of output and production factors available, under a given level of technology:

$$
\begin{gathered}
Y=f(K, L, A), \text { where } \\
Y \text { - output, } \\
L \text { - labor input, } \\
K \text {-capital input, } \\
A \text { - technology. }
\end{gathered}
$$

Analogously to the national level, the production function can be computed for every region. Then the decomposition of growth in every region's output is made proceeding from the differential form of the production function:

$$
\frac{\dot{Y}}{Y}=g_{T F P}+\left(\frac{F_{K} \cdot K}{Y}\right) \cdot\left(\frac{\dot{K}}{K}\right)+\left(\frac{F_{L} \cdot L}{Y}\right) \cdot\left(\frac{\dot{L}}{L}\right),
$$

where:

$F_{K}$ and $F_{L}$ - marginal products of capital and labor production factors, respectively,

$g_{\text {TFP }}$ - growth rate determined by technical progress:

$g_{\text {TFP }} \equiv\left(\frac{F_{A} \cdot A}{Y}\right) \cdot\left(\frac{\dot{A}}{A}\right)$ (in the case of the neutral technical progress $g_{\text {TFP }} \equiv\left(\frac{\dot{A}}{A}\right)$ ).

The pace of growth of technical progress is computed as a remainder:

$$
g_{\text {TFP }}=\frac{\dot{Y}}{Y}-\left(\frac{F_{K} \cdot K}{Y}\right) \cdot\left(\frac{\dot{K}}{K}\right)-\left(\frac{F_{L} \cdot L}{Y}\right) \cdot\left(\frac{\dot{L}}{L}\right) .
$$


Thus received growth rates of technical progress are usually defined as assessment of growth of total factor productivity ${ }^{26}$.

Finding empirical assessments of $g$ necessitates the knowledge of values of marginal products $F_{K}$ and $F_{L}$. In an assumption of constant returns to scale and competitive markets for inputs and the final product, marginal products can be assessed on the basis of observed:

$w$ - price of labor services,

$R$ - rental price of capital.

In this event the total factor productivity growth index is computed as follows:

$$
\ln \left(\frac{A_{t}}{A_{t-1}}\right)=\ln \left(\frac{Y_{t}}{Y_{t-1}}\right)-\left(\alpha_{L} \ln \left(\frac{L_{t}}{L_{t-1}}\right)+\alpha_{K} \ln \left(\frac{K_{t}}{K_{t-1}}\right)\right),
$$

where

$\bar{\alpha}_{L}$ and $\bar{\alpha}_{K}$ - values of shares of the respective factors in output averaged over two periods:

$$
\begin{aligned}
& \bar{\alpha}_{L}=\frac{\alpha_{L}(t)+\alpha_{L}(t-1)}{2} ; \alpha_{L}=\frac{w L}{Y}, \\
& \bar{\alpha}_{K}=\frac{\alpha_{K}(t)+\alpha_{K}(t-1)}{2} ; \alpha_{K}=\frac{R K}{Y}{ }^{27} .
\end{aligned}
$$

\footnotetext{
${ }^{26}$ Different papers on decomposition of growth provide various definitions of the remainder: while Solow (1957) considers it measure of technical progress, Harberger (1998) - views it as real cost reduction, Abramovitz (1993) notes that the remainder comprises totality of unmeasured sources of growth and call it "some sort of measure of ignorance".

${ }^{27}$ In the general case of the neoclassical production function, the equation is met approximately, while a precise equation is possible only for the translogarythmic production function. (Diewert, 1976).
} 
The computation of the weights is based upon the condition: $Y=R \cdot K+w \cdot L$ or $\alpha_{K}+\alpha_{L}=1$, which is met providing the whole output is conditioned solely by factors included in the production function (i.e. it suggests constant return to of scale).

\subsubsection{Assessing Output}

The indicators that mirrors changes in output can be represented by volume of output (volume of production of goods and services), national income (NI), and gross domestic product (GDP). While opting for a certain measure of output, a researcher, as a rule, is guided by research objectives and models he deals with, on the one hand, and on-hand statistical data, on the other.

The choice between national income and gross domestic product appears rather conditional. The differences between TFP assessments received basing on the indicators are determined by changes in the volume of consumption of fixed assets. While on the one hand, if the major purpose of an analysis is to expose causes and potential possibilities for boosting output, the change in the volume of consumption of fixed assets should be ignored, while building the TPF assessments (Denison (1972)). On the other hand, the preference of net output generates differences in identification of the basic factors - whole under the assessment of factor services capital consumption is excluded, the depreciation of labor, which appears an indeterminable value in the general case, is included in the volume of labor services (Griliches, Jorgenson (1967)).

The employment of every single indicator as a measure of output imposes its constraints on production function and, consequently, on an interpretation of TFP. For example, differences in assessments of TFP received on the basis of final output and other indicators is determined by the volume of intermediate consumption and the output prices to intermediate consumption ratio (thus, under the constant proportion of intermediate consumption Y (N / 
$\mathrm{Y}=\mathrm{n}=$ const) TFP growth rates on the basis of final output appear $\frac{1}{1-n}$ times greater than assessments received on the basis of GVA (Bruno (1982)).

In international practice (OECD (2001a, 2001b)), assessments of TFP on the basis of final output are used, as a rule, under the analysis of changes in productivity on the industry or sectoral levels. The definition of productivity on the basis of value added is more frequently employed to analyze connections on the microand macrolevels - for instance, to study the contribution of individual industries to the change in TFP by an economy as a whole, and to study structural changes.

The present paper considers gross regional product (GRP) as a measure of output, while to build assessments on the industry level (the industrial sector), the authors employ volume of industrial output (VIO).

For the sake of decomposition of growth, the respective indicators should be translated into comparable prices. The process of computation of deflators forms a separate complex task and because of the absence of the necessary volume of statistical information, it is not considered in the present paper. Let us just note that from the theoretical perspective, the computation of the respective deflators should involve hedonic indices that consider change in the quality of a given produce and inputs over time.

The Russian Statistical Service (Rosstat) provides the indicator of growth in the physical volume of regions' GRP (GVA). To compute GRP, regional statistical bodies employ a uniform approved methodology. Regretfully, the information of a real growth of regions' GRPs is available only starting from 1997, which considerably shortens the interval for the research. Let us note that results of decomposition of growth appear extremely sensitive to the choice of deflators. In the situation when information of compo- 
nents of output and prices is unavailable, the official statistics on the real dynamics of GRP form a priority (at least, for the purpose of decomposition of growth).

To extend a possible interval of research, we have tried to approximate the dynamics of the real volume of GRP on the basis of the GDP deflator and the regions' consumer price index (CPI). In the former case, there appears the necessity to introduce a prerequisite of GRP deflators being equal for all the regions, which appears poorly matched to the reality. The employment of regional CPIs as GRP deflators leads to lowering the assessment of GRP growth rates vis-à-vis the official data on the dynamics of the physical volume of GRP. The comparison of results of the computations based on both methods with the official data on growth rates for the federal districts are given in Table 3.1. The comparison of results of the computation of the index of output across regions is given in Annex (table A2-3). Because of a great discrepancy between assessments, in the paper below, the decomposition of growth was conducted for the period between 1997 and 2002, on which the official statistics on the dynamics of regional output are available.

For most federal okrugs (Fig. 3.1) the dynamics of the output (GRP) index are similar to those of GDP index for Russia as a whole. The indices sunk in 1997-98 (with the greatest decline in the Siberian okrug whose 1998 output fell by $12.5 \%$ vs. the 1996 one). The trend changed in 1998-99 (with the output rising constantly in all the federal okrugs). In the Central okrug, during the whole period in question the output remained at a level greater than its 1996 index, while the Volga, North-Western and Southern okrugs managed to reach the 1996 level as early as in 1999, the Ural in 2000, and the Far-Eastern and Siberian ones - only in 2001.

The dynamics of regional indices (see Annex 2, Table A2-4) likewise allow singling out two stages: namely, the fall in output and its rise (Fig. 3.2). In some regions, the change of the trend occurred ear- 
lier than in 1999: thus, in Astrakhan and Orel oblasts, the rise in output is noted through the whole period of 1997-200; in Republic of Kabardino-Balkaria, Republic of North-Ossetia - Alania, Tver oblast, the rise in output started yet in 1998. By contrast, in Bryansk, Magadan, Tula oblasts, Republic of Karachaevo-Cherkessia, Altay republic, republics of Ingoushetia, kalmykia, Komi, Sakha and Khakassia, the change of the periods occurred in 2000.

Table 3.1

Results of Computations of GRP Growth Indices by Federal Okrugs on the Basis of Different Deflators

\begin{tabular}{|c|c|c|c|c|c|}
\hline \multicolumn{6}{|c|}{ The 2002 GRP Index Value $(1996 .=100)$} \\
\hline Federal Okrug & $\begin{array}{l}\text { Variant1: Index } \\
\text { of physical } \\
\text { volume of GRP }\end{array}$ & $\begin{array}{c}\text { Variant 2: } \\
\text { Computation } \\
\text { on the basis of } \\
\text { GDP deflator }\end{array}$ & $\begin{array}{c}\text { Variation (as \% } \\
\text { of variant 1) }\end{array}$ & $\begin{array}{c}\text { Variant 3: } \\
\text { computation on } \\
\text { the basis of } \\
\text { regions' CPI }\end{array}$ & $\begin{array}{l}\text { Variation (as \% } \\
\text { of variant 1) }\end{array}$ \\
\hline Far-Eastern & 109.33 & 96.35 & -11.88 & 104.87 & -4.08 \\
\hline Volga & 118.87 & 95.23 & -19.89 & 87.57 & -26.33 \\
\hline North-Western & 126.45 & 114.22 & -9.67 & 114.72 & -9.28 \\
\hline Siberian & 109.48 & 84.22 & -23.07 & 91.64 & -16.30 \\
\hline Ural & 121.31 & 105.80 & -12.79 & 97.94 & -19.27 \\
\hline Central & 137.32 & 146.57 & 6.74 & 135.37 & -1.42 \\
\hline Southern & 129.01 & 108.46 & -15.93 & 104.77 & -18.79 \\
\hline $\begin{array}{l}\text { Across RF as a } \\
\text { whole }\end{array}$ & 123.95 & 111.61 & -9.95 & 106.33 & -14.21 \\
\hline
\end{tabular}

In Belgorod, Moscow, Murmansk, Perm, Tambov oblasts, city of Moscow, Republic of Mordovia, despite decline in their output in 1998, its values have not ever sunk below the 1996 level, while the Jewish autonomic oblast, Irkutsk, Kamchatka, Magadan oblast, republics of Adygea, Mary-El, Khakassia by 2002 have failed to reach the 1996 level. 


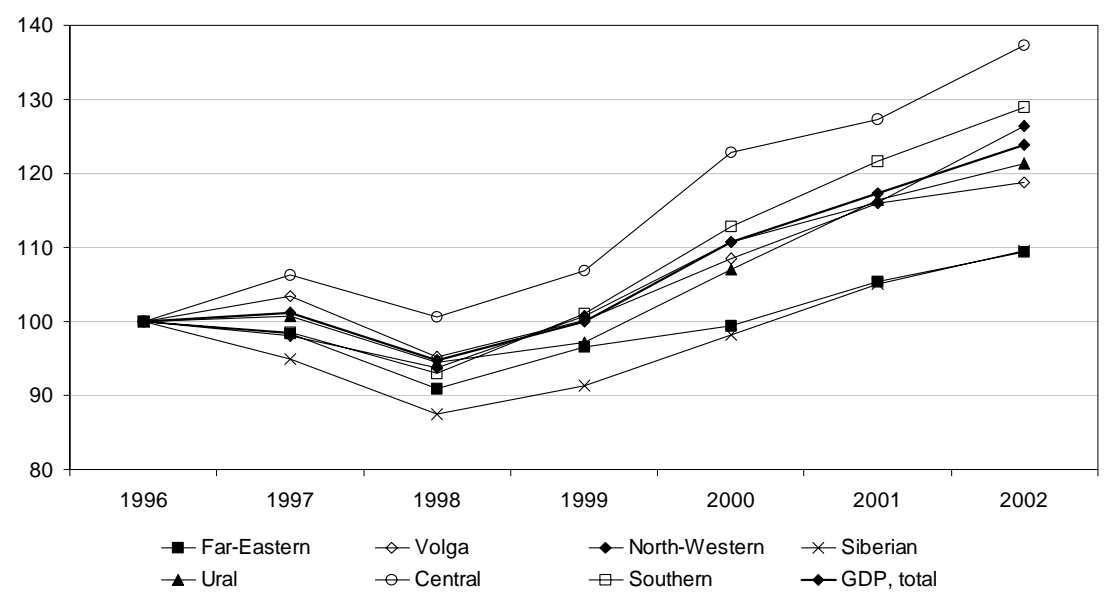

Fig. 3.1. The Dynamics of Indices of Output across Federal Okrugs $(1996=100 \%)$

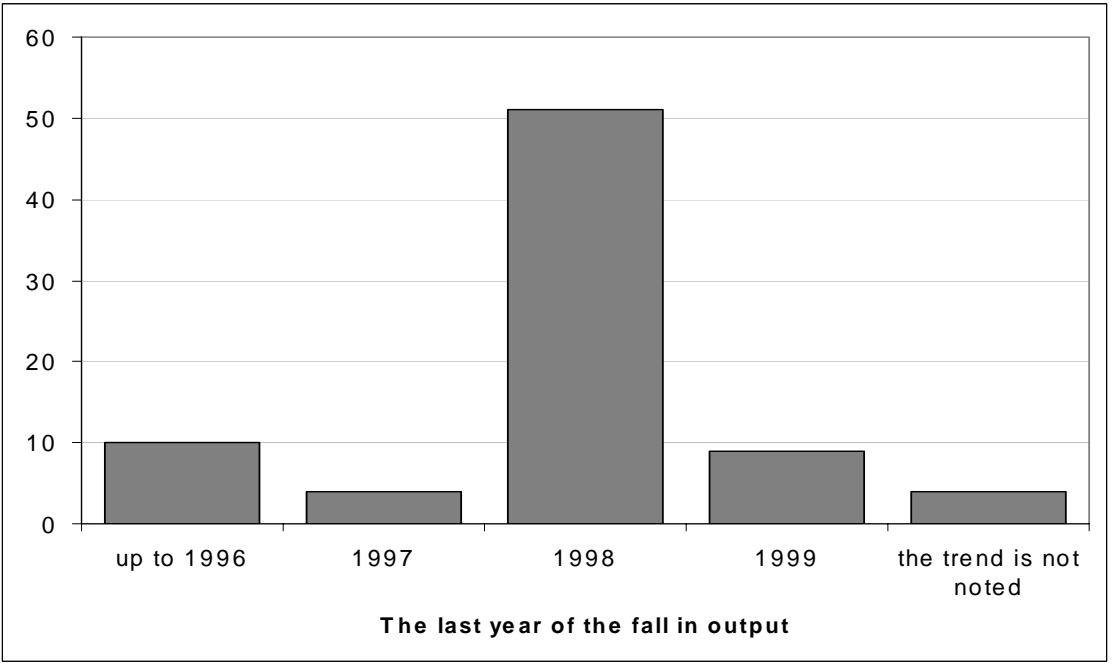

Fig. 3.2. Bar Chart of the Moment of the Change of the Trend in the Dynamics of Regions' Output 
The pictorial rendition of the regional differentiation of the change in output volumes can be represented by means of Sun-rise diagram $^{28}$ (see Fig. 3.3 and 3.4). As Fig. 3.3 and 3.4 show, in 1997-2002 the rise in GDP across the federal okrugs can be considered relatively more even than across regions. This is determined by the fact that all the federal okrugs demonstrated positive growth rates over the period in question, while there are regions with negative growth rates (republics of Adygea, Khakassia, Mary El, Ingoushetia, Jewish autonomic oblast, Irkutsk, Magadan, Kamchatka oblasts). The contraction of the regions' GRPs (given that in the initial period (1996) their share in GDP had accounted roughly for $3.9 \%$ ) made up $-1.7 \%$ of the aggregate rise in GDP.

${ }^{28}$ A special case of Lorenz curve. Harberger (1998) employed the definition to analyze the irregularity of real cost reduction in sectoral terms. Such diagrams are built as follows: the horizontal axe (in our case) presents the regional structure of GDP as a cumulative result. Regions are ranked according to their growth rates with the region enjoying the greatest growth rates ranked the first and the one with the smallest rate - the last. The vertical axe presents growth rates of the respective regions. The more convex the curve that unites the points is, the more uneven in regional terms growth is. Accordingly, an uneven growth would be represented by a straight line. 


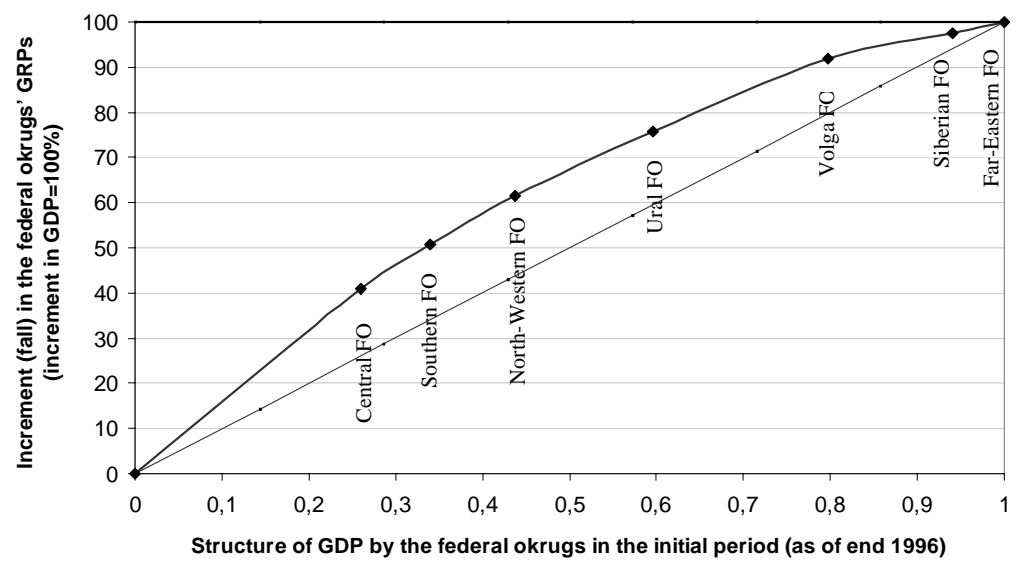

Fig 3.3. Sun-Rise Diagram of Growth in GDP (1997-2002.) at the Level of the Federal Okrugs

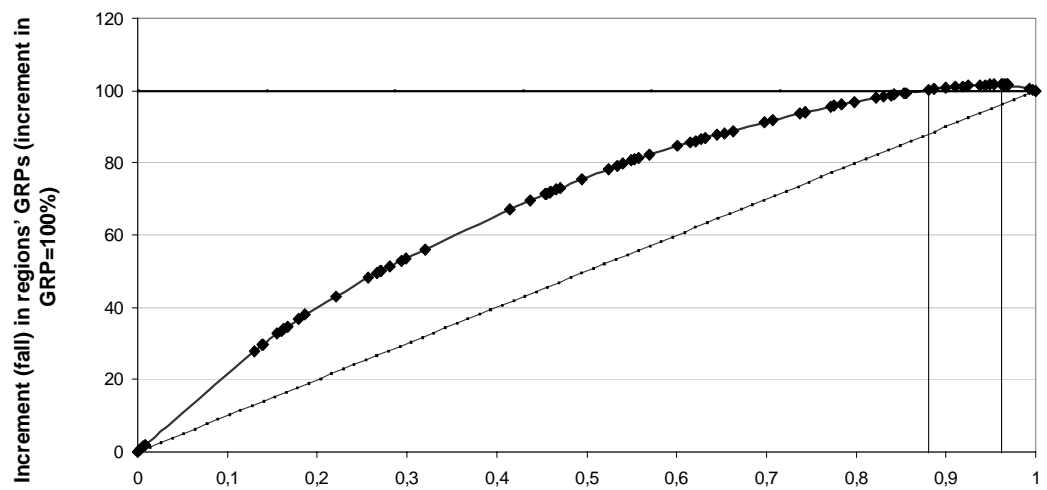

Regional structure of in the initial period (as of end 1996)

Fig. 3.4. Sun-Rise Diagram of Growth in GDP (1997-2002) at the Regional Level 


\subsubsection{Assessing Labor Input}

The concept of contribution of the labor factor to economic growth, implies, as a rule, labor services provided by working population. The simplest measure of labor input can be worked man-hours. However, it is known that labor forms a nonhomogenous indicator and it depends, as a minimum, on employees' qualification and a number of other factors (gender, age, sectoral profile, etc.) In his first papers, Denison (1962) demonstrated that the contribution of labor could change even if the total number of worked man-hours was constant. That is why to ensure a more accurate measuring, one needs a detailed breakdown of the used labor by categories, with account of worked hours and marginal productivity.

However, it was just recently that Rosstat has begun providing information of worked hours ${ }^{29}$ on the regional level, which substantially reduces the horizon of the analysis. It is possible to apply an alternative method of assessing the labor employed in the production process basing on the available employment statistics $\left(N^{i}\right)$. The dynamics of employment indices by the federal okrugs are given in Fig. 3.4. However, the use of employment instead of worked hours affects the interpretation of the TFP indicator. In the event labor input is assessed on the basis of the indicator of the number of employees, the unexplained by factors growth (assessment of TFP) comprises a component that corresponds to qualitative changes in labor input (changes in the age and gender structure, employees' educational level and qualification, distribution of labor resources by sectors, among others).

The comparison of the employment and output dynamics displays that in the period in question (1997-2002), the range of

${ }^{29}$ Obsledovanie naselenia po problemam zanyatosti, Rosstat, 1999-2002. 
changes in the employment index across the federal okrugs did not exceed 12\% against the 55\% range of changes in GRP (see Fig. 3.5 and 3.1)

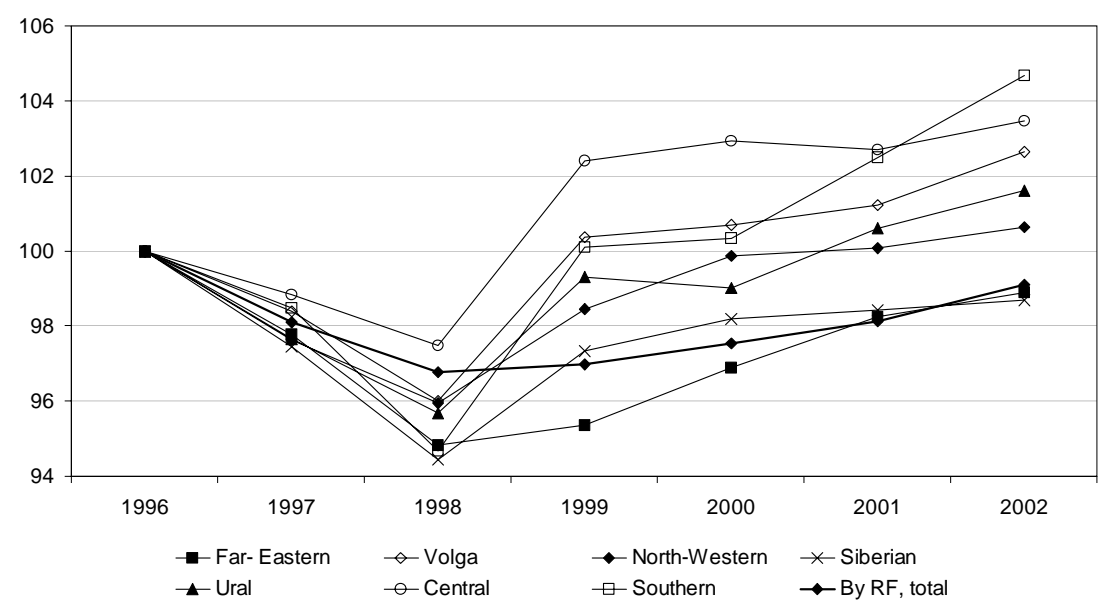

Fig. 3.5. The Dynamics of Employment Indices by the Federal Okrugs $(1996=100 \%)$

The contribution of labor is computed as a product of multiplication of the labor input weight coefficient by their growth rate; the 1997-2002 results and two sub-periods are given in Table 3.2.

As the Table shows, it was until 1998 that labor input ensured the greatest contribution to output. Against the background of the declining output, the contraction in labor input has been happening because of an intense discharge of labor force (lay-offs) ${ }^{30}$. On the contrary, there was no drastic rise in employment over the second period in question. A considerable rise in output was noted under rather a modest growth in employment. That occurred apparently

\footnotetext{
${ }^{30}$ The present paper is not intended to research into the causes for the contraction in employment, which could be initiated by either party.
} 
thanks to a more intense consumption of then remaining labor resources (just a reminder, the indicator of factor input is employment, rather than worked hours, which enables us to advance this assumption).

Table 3.2

Results of Assessments of Growth Rates in Output and Average Annual Number of Employment by Federal Okrugs

\begin{tabular}{|c|c|c|c|}
\hline \multicolumn{4}{|c|}{ 1997-1998 } \\
\hline Federal okrug & $\begin{array}{l}\text { GRP growth } \\
\text { rate }\end{array}$ & $\begin{array}{l}\text { Labor reserves } \\
\text { growth rate }\end{array}$ & $\begin{array}{l}\text { Labor reserves growth rate as } \\
\% \text { of the GRP growth rate }\end{array}$ \\
\hline Far-Eastern & -4.80 & -1.93 & 40.15 \\
\hline Volga & -2.63 & -0.98 & 37.40 \\
\hline North-Western & -3.22 & -1.31 & 40.71 \\
\hline Siberian & -6.69 & -1.71 & 25.50 \\
\hline Ural & -2.92 & -1.07 & 36.58 \\
\hline Central & 0.12 & -0.60 & -496.82 \\
\hline Southern & -3.66 & -1.53 & 41.77 \\
\hline \multicolumn{4}{|c|}{ 1999-2002 } \\
\hline Far-Eastern & 4.60 & 0.69 & 15.08 \\
\hline Volga & 5.52 & 0.80 & 14.42 \\
\hline North-Western & 7.46 & 0.67 & 8.93 \\
\hline Siberian & 5.60 & 0.63 & 11.23 \\
\hline Ural & 6.22 & 0.71 & 11.39 \\
\hline Central & 7.72 & 0.63 & 8.15 \\
\hline Southern & 8.17 & 1.34 & 16.42 \\
\hline \multicolumn{4}{|c|}{ 1997-2002 } \\
\hline Far-Eastern & 1.37 & -0.19 & -13.77 \\
\hline Volga & 2.73 & 0.20 & 7.32 \\
\hline North-Western & 3.77 & 0.00 & 0.08 \\
\hline Siberian & 1.33 & -0.16 & -11.75 \\
\hline Ural & 3.08 & 0.11 & 3.66 \\
\hline Central & 5.13 & 0.22 & 4.24 \\
\hline Southern & 4.07 & 0.38 & 9.22 \\
\hline
\end{tabular}


Overall, employment ensured rather a small proportion of GRP growth rates over 1997-2002, with the rise in output in the FaEastern and Siberian okrugs being accompanied by contraction in the average annual number of employees.

The structure of labor resources in regions changes over time. As the volume of output produced by unit of labor input (i.e. the quality of labor) varies across groups of labor resources, one cannot help but being taken into account while building TFP assessments. The assessment of the impact of structural changes is based upon the assumption that the average wages in each group is proportional to the marginal labor product per employee (in the case for assessment of labor reserves) or per worked hour (in the case for assessment of labor services (Denison, 1967).

The building of the assessment of labor input that considers differences in the structure of labor resources involved in the production process necessitates data on distribution of employees across the groups. In the absence of the respective statistics, all kinds of labor inputs, regardless of their classification, are aggregated on the basis of the overall proportion of labor input in output $\frac{\sum R_{j} \cdot L_{j}}{Y}$. The assessment of total factor productivity in this case takes the form: $\hat{g}_{T F P}=g_{Y}-\alpha_{K} \cdot g_{K}-\left(\frac{\sum w_{j} \cdot L_{j}}{Y}\right) \cdot\left(\frac{\dot{L}}{L}\right)$. As a result, the bias of such an assessment from the one that considers differences in the structure of labor resources makes up: $\hat{g}_{\text {TFP }}-g_{\text {TFP }}=\left(\frac{\sum w_{j} \cdot L_{j}}{Y}\right) \cdot\left(\frac{\dot{L}}{L}\right)-\sum \alpha_{L_{j}}\left(\frac{\dot{L}_{j}}{L_{j}}\right)$. For example, in the case two groups of employees are singled out- 
$\hat{g}_{\text {TFP }}-g_{\text {TFP }}=\frac{L_{1}}{L_{1}+L_{2}} \cdot \frac{L_{2}}{L_{1}+L_{2}} \cdot \frac{L_{1}+L_{2}}{Y} \cdot\left(w_{1}-w_{2}\right) \cdot\left(\frac{L_{1}}{L_{1}+L_{2}}-\frac{\dot{L}_{2}}{L_{1}+L_{2}}\right)$,

i.e. if $w_{1}>w_{2}, \frac{\dot{L}_{1}}{L_{1}+L_{2}}>\frac{\dot{L}_{2}}{L_{1}+L_{2}}$, then $\hat{g}_{\text {TFP }}>g_{\text {TFP }}$.

Consequently, the assessment of total factor productivity appears overvalued, as it comprises the effect from the rise of the employees' qualification (Barro (1998)) in the event of singling out groups of employees by age, gender, educational level, or the impact of a redistribution of resources in the event of singling out groups of employees by kinds of job, industry.

The assessment of labor input on the basis of the indicator of the overall number of employees in region $N^{i}$ suggests that all the employees in the region form a homogenous group. Lifting this restriction suggests the transition to disaggregated assessments:

$$
N^{i} \Rightarrow N_{\text {ind }}^{i}=\sum_{j} \frac{w_{j}^{i}}{\bar{w}^{i}} N_{j}^{i},
$$

where

$w_{j}^{i}$ - labor compensation of $\mathrm{j}$ category of employees in i region,

$\bar{w}^{i}$ - average wages in i region,

$N_{j}^{i}$ - the number of employees of $\mathrm{j}$ category in i region.

A transition from $N^{i}$ to $N_{\text {ind }}^{i}{ }^{31}$ does not lead to substantial changes in the dynamics of the labor input index. The employment index with account of sectoral variations (Fig. 3.6) for most federal okrugs demonstrates decline in 1997-1998 (except for the FarEastern okrug where the decline was over in 1999 and the Ural one

${ }^{31}$ According to the statistics available, employees in a region are divided into categories only according to their sectoral attribution. 
in which it did not occur at all). Given that, in all the federal okrugs, except the Ural one, the consequent rise in the employment index with account of sectoral differences proved to be lower than the rise in the employment index.

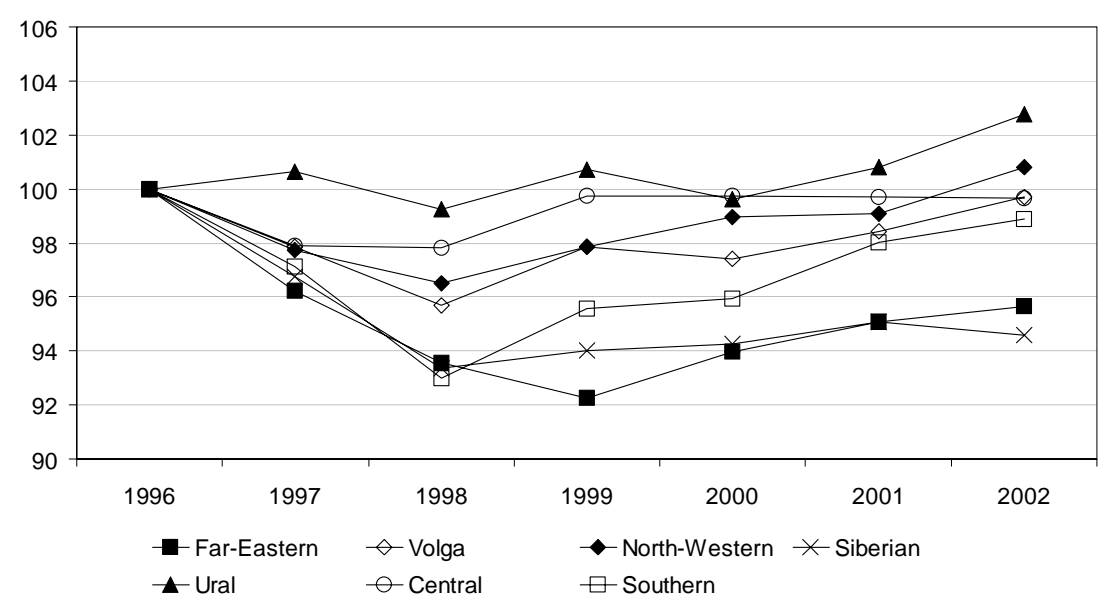

Fig. 3.6. The Dynamics of Employment Indices of the Federal Okrugs with Account of Sectoral Differences $(1996=100 \%)$

A less notable fall in the assessment of labor input with account of sectoral structure (Fig. 3.6) vis-à-vis employment-based assessment (Fig. 3.5) in a number of okrugs evidences that in the okrugs there happened an intense redistribution of labor force between sectors. Thus, the Ural okrug has not practically seen reduction of labor input, while employment had been contracting there by 1998 . By contrast, according to the assessment based on sectoral differences, in the Far-Eastern okrug the fall in labor input was yet more visible than the contraction in employment.

Having transformed the expression for assessing the contribution of labor reserves to the rise in output in $i$ region, we arrive at: 


$$
\alpha_{L}^{i} \frac{\dot{L}^{i}}{L^{i}}=\alpha_{L}^{i} g_{N_{\text {ind }}^{i}}=\alpha_{L}^{i} g_{\sum_{j} w_{\frac{w^{i}}{i}}^{i} N_{j}^{i}}=\alpha_{L}^{i}\left(g_{N^{i}}+g_{\sum_{j} \frac{w_{j}^{i} N_{j}^{i}}{\bar{w}^{i}} N^{i}}\right)
$$

Table 3.3

\section{Results of the Assessment of Output growth Rates and Contribution of Components of the Assessment of Labor Reserves across the Federal Okrugs}

\begin{tabular}{|c|c|c|c|c|c|c|c|}
\hline \multirow[b]{2}{*}{ Federal okrug } & \multicolumn{4}{|c|}{ Growth rates } & \multicolumn{3}{|c|}{ As $\%$ of the GRP growth rates } \\
\hline & $\begin{array}{l}\text { Number of } \\
\text { employed }\end{array}$ & $\begin{array}{l}\text { Structure of } \\
\text { employed }\end{array}$ & Labor input & GRP & $\begin{array}{l}\text { Number of } \\
\text { employed }\end{array}$ & $\begin{array}{c}\text { Structure of } \\
\text { employed }\end{array}$ & Labor input \\
\hline \multicolumn{8}{|c|}{ 1997-1998 } \\
\hline Far-Eastern & -1.93 & -0.51 & -2.44 & -4.80 & 40.15 & 10.70 & 50.82 \\
\hline Volga & -0.98 & -0.08 & -1.06 & -2.63 & 37.40 & 3.05 & 40.44 \\
\hline North-Western & -1.31 & 0.19 & -1.12 & -3.22 & 40.71 & -5.78 & 34.93 \\
\hline Siberian & -1.71 & -0.34 & -2.05 & -6.69 & 25.50 & 5.07 & 30.56 \\
\hline Ural & -1.07 & 0.88 & -0.19 & -2.92 & 36.58 & -30.11 & 6.46 \\
\hline Central & -0.60 & 0.07 & -0.53 & 0.12 & -496.82 & 59.72 & -436.95 \\
\hline Southern & -1.53 & -0.51 & -2.04 & -3.66 & 41.77 & 13.97 & 55.69 \\
\hline \multicolumn{8}{|c|}{ 1999-2002 } \\
\hline Far-Eastern & 0.69 & -0.33 & 0.36 & 4.60 & 15.08 & -7.17 & 7.88 \\
\hline Volga & 0.80 & -0.30 & 0.50 & 5.52 & 14.42 & -5.45 & 9.03 \\
\hline North-Western & 0.67 & -0.04 & 0.63 & 7.46 & 8.93 & -0.48 & 8.47 \\
\hline Siberian & 0.63 & -0.45 & 0.18 & 5.60 & 11.23 & -8.05 & 3.24 \\
\hline Ural & 0.71 & -0.29 & 0.42 & 6.22 & 11.39 & -4.67 & 6.75 \\
\hline Central & 0.63 & -0.43 & 0.20 & 7.72 & 8.15 & -5.62 & 2.59 \\
\hline Southern & 1.34 & -0.52 & 0.82 & 8.17 & 16.42 & -6.42 & 10.08 \\
\hline \multicolumn{8}{|c|}{ 1997-2002 } \\
\hline Far-Eastern & -0.19 & -0.39 & -0.58 & 1.37 & -13.77 & -28.64 & -42.51 \\
\hline Volga & 0.20 & -0.23 & -0.02 & 2.73 & 7.32 & -8.32 & -0.89 \\
\hline North-Western & 0.00 & 0.04 & 0.04 & 3.77 & 0.08 & 1.01 & 1.14 \\
\hline Siberian & -0.16 & -0.41 & -0.57 & 1.33 & -11.75 & -31.08 & -42.62 \\
\hline Ural & 0.11 & 0.10 & 0.22 & 3.08 & 3.66 & 3.17 & 7.03 \\
\hline Central & 0.22 & -0.27 & -0.04 & 5.13 & 4.24 & -5.18 & -0.85 \\
\hline Southern & 0.38 & -0.52 & -0.14 & 4.07 & 9.22 & -12.76 & -3.43 \\
\hline
\end{tabular}


Thus the contribution of labor reserves to the output growth rates in $i$ region can be presented in the form of two components: (1) $\alpha_{L}^{i} g_{N^{i}}$ that considers the impact of changes in the number of employees and (2) $\alpha_{L}^{i} g_{\sum_{j} \frac{w_{j}^{i} N_{j}^{i}}{\bar{w}^{i} N^{i}}}$ that determines the impact of changes in the structure of employees. Given the above, coefficient $\alpha_{L}^{i}$ remains unchanged for the whole period.

The calculations witness (Table 3.3) that the changes in the sectoral structure of the employed have resulted in the rise in the proportion of the output growth rates explained by changes in labor input in most federal okrugs over the 1997-98 period of decline. The period of growth of 1999-2002 displays an opposite situation: most federal okrugs saw the contribution of labor input to the output growth rate lessen.

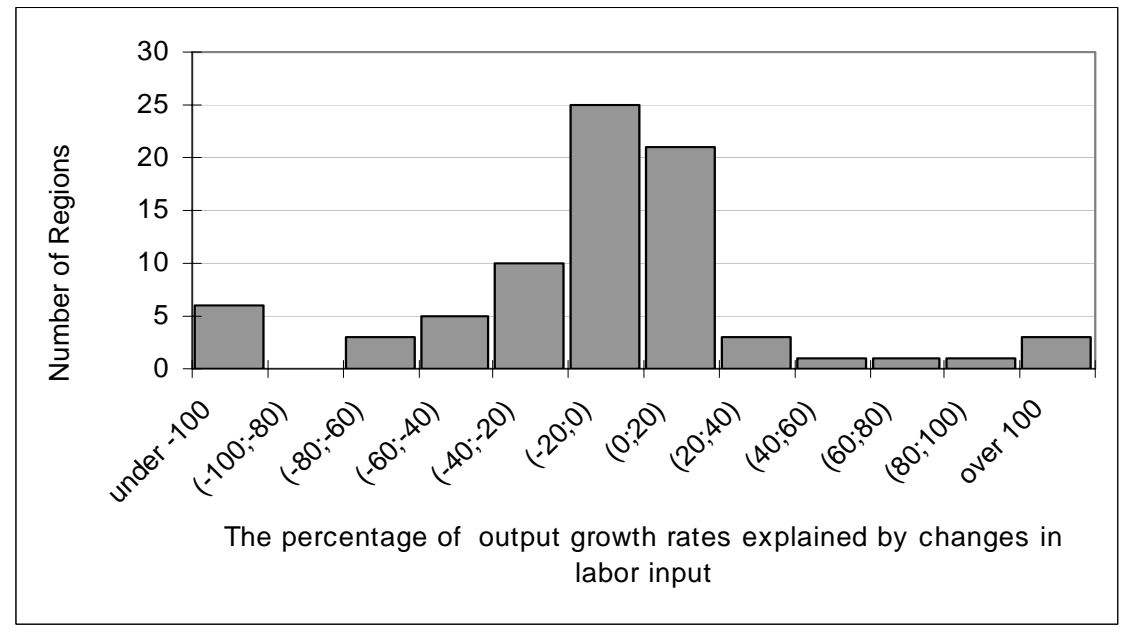

Fig.3.7. Bar Chart of the Percentage of the Output Growth Rates Explained by Changes in the Average Number of Employed in Regions with Account of Sectoral Differences (for the Period of 1997-2002) 
The percentage of the output growth rate explained by changes in labor input varies substantially across regions. For some of them, the direction of changes of the output growth rate appears opposite to the direction of changes in the labor input growth rates (negative values in the Bar Chart). However, for most regions changes in the average number of the employed with account of industry-specific differences explain from $-20 \%$ to $20 \%$ on average of the GRP growth rates (see Fig. 3.7). A great percentage of negative assessments of the contribution of labor input to growth testifies either to an insignificant role played by the labor factor in the regional growth over the period in question, or a poor accuracy of measure of the respective indices. Yet another reason for the presence of negative assessments lies with the fact that one can single out two stages of the time interval in question that concern both the dynamics of output and employment: namely, decline and growth stages. For most regions and the federal okrugs (see Fig. 3.1 and Fig. 3.6) the 1997-1998 decline in output was just slightly in excess of the one in employment (at $2 \%$ on average), while the consequent rise in output in 1999-2002 substantially outpaced the rise in employment (by $25 \%$ on average). The computed averaged (over the whole period between 1997 and 2002) growth rates of the indicators do not mirror such changes in the dynamics. Meanwhile, for the sub-periods (see Annex 2, Fig. A2-3 and Fig. A2-4) the proportion of the output growth rates explained by changes in labor input has shifted to the area of positive values: while in 1997-98 it accounted for $20-40 \%$ on average, in 1999-2002 - 0-20\%.

To ensure a more accurate assessment of labor input, one should take into account worked time (thus differing from the number of the employed), the employees' gender and age structure, their qualification and education. 


\subsubsection{Assessing Capital Input}

Issues associated with capital input assessments pose the greatest problems not only in the framework of Russian statistics but to research into decomposition of growth on the whole.

An assessment of capital input in growth decomposition models gives a rise to a number of issues that concern the methodology of assessment of the input itself and assessment of the measure of its quality. Production capacities of different generations (vintages) are undoubtedly characterized by different productivity rates. Economists disagree on the issue as to how one needs to exercise the procedure (which indicators to use as weights while building an aggregate assessment) - whether they have to employ relative prices or marginal products.

To assess capital input, Denison $(1967,1974)$ employed the sum of production units weighted on the basis of their relative prices in the basic period, while he regarded the unmeasured improvement of quality of the capital as a component of the contribution by technical progress. His stand (Denison $(1978,1980))$ is that while conducting decomposition, one can neglect the "embodiment" hypothesis, as changes in the equipment age structure have just a minor effect on the output growth rates, even if one considers that all technical progress has found its embodiment in capital. In their paper, Gregory and Denis (1973), as well as Phelps in his theoretical paper (1982), arrive at the same conclusion.

However, models that suggest consideration of technical progress embodied in capital have recently begun earning an increasing popularity. In this particular case, it is assumed that new equipment has a greater productivity than the one placed in operation earlier, and capital index is built on the basis of marginal products, whose approximation is formed by prices of the factors (Griliches, Jorgenson (1967)). The "embodiment" hypothesis has found its reflection in many papers which regard technical progress 
as function of the investment rate (Kaldor (1957)); the rate of change of the investment rate (Kaldor, Mirrlees (1962)); as a factor that determines prices of investment goods (Griliches (1961), Brubaker (1968), Hall (1968), Gordon (1990)).

The compromise solution is formed by a model of sources of growth that considers technical progress, both embodied and not embodied in capital. This concept was developed by Nelson (1964), Jorgenson (1966), Hulten (1992) on the basis of assessments of changes in the quality of the basic factors.

The two approaches to interpretation of technological changes suggest different interpretations of capital. In the former case (technical progress is assumed not to be embodied in capital), the differences between production capacities of different generations are limited by some factor associated with the depreciation level, therefore:

$$
K(t)=\sum_{\tau=0}^{t}(1-\delta)^{\tau} I(t-\tau),
$$

where $\delta$ - deterioration rate (productivity loss in the process of ageing of the equipment).

If investment volumes $I(t)$ of period $t$ are measured by the number of production units, then assessment $K(t)$ received as a weighted sum of previous investment constitutes the number of production units in an equivalent $t$.

In the assumption of technical progress embodied in capital, such an assessment leads to the fall of an actual effect from the capital reserves productivity. This defect can be liquidated (Fischer (1965)) by introducing the technical efficiency index whose changes are conceived as qualitative differences between production capacities of different age (Hulten (1992)), i.e. the growth rate 
of $\Phi(t)$ constitutes the growth rate of technical progress embodied in capital. In this case

$$
\hat{K}(t)=\sum_{\tau=0}^{t}(1-\delta)^{\tau} I(t-\tau) \Phi(t-\tau) .
$$

Excessive depreciation forms yet another critical source of biases in the course of assessing capital. Numerous empirical research papers note that it is moral ageing of equipment that forms a more important characteristic of depreciation and not its physical depreciation. Economically, capital ages faster than its operational deadline comes, nonetheless the outdated equipment do not fully loose its capability to generate output. So the value of services the capital provides falls over time at a speed different from that of depreciation write-offs. In the event net investment and depreciation are equal, the net capital formation equals zero, while in the conditions of improvement of the level of technologies, the volume of capital grows (in the frame of the "embodiment" hypothesis). On the other hand, if one does not consider the rise of efficiency of the capital in conjunction with its age, while assessing capital input, one should also ignore its moral ageing. As a result, the assessment of capital on the basis of net value (less depreciation) that does not consider technical progress embodied in the capital leads to an undervalued assessment of its impact on economic growth rates.

So, the theoretical assessments of fixed assets should represent an aggregate indicator that considers changes in the quality of individual production units associated both with their ageing and depreciation, and the change of the technological level. The building of such an assessment necessitates information of assets being placed into operation, their productivity in comparison with already existing ones, their operational term, maintenance and repair costs, and numerous other factors (see (3.7)-(3.8)). 
The statistics of the national wealth is one of the weakest spots of Russian statistics. At the conference "Assessment and Management of the National Wealth in Russia" (Ivanov, Khomenko (2003)), its participants noted that the absence of the respective information base and the mismatch between the principles of Russian accounting system and those of the National Accounting System posed the critical problem in this area. In Russian accounting system, fixed assets are often assessed basing on their initial price, rather than on prices in existence as of the moment of compiling reports; the detailed information of the structure of fixed assets is lacked as well. In addition, revaluations of fixed assets that have become compulsory since 1997 also introduce considerable biases.

The currently existing drawbacks of the national wealth statistics, as well as of the statistics of fixed assets of the Soviet period that form the starting point for computations of their dynamics seriously complicate the task of assessing actual changes in volumes of production capacities. According to the official statistical data, the physical volume of fixed assets grew by 5.99\% between 1999 and 2001; according to some estimates (Khanin (2005)), fixed production assets fell by $30 \%$ over the period concerned, while their active part contracted by 50-60\%; according to the IET experts (Entov, Lugovoy at al. (2003)) Russia's fixed assets shrank by $12 \%$ on average, while effective ones - 2.9 times on average.

At the regional level, the statistical data on fixed assets appeared yet poorer. The practically available regional indicator that regional government statistical bodies provide is the total book value of fixed assets, which, due to fixed assets revaluations, poorly mirrors actual processes of formation of enterprises' production capacities in a given region. For example, the dynamics of FA indices (computed on the basis of FA value given in constant prices on the basis of the GDP deflator averaged as of the start and end of a given pe- 
riod) across the federal okrugs (Fig. 3.8) demonstrates a drastic rise in 1997 and a dramatic decline afterwards.

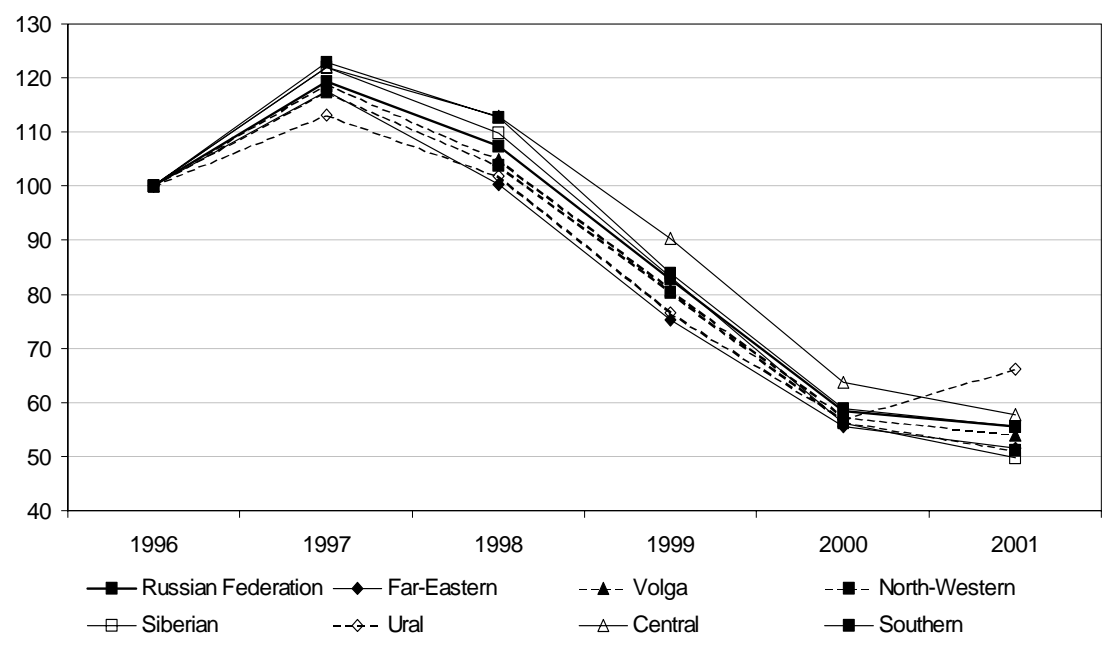

Fig. 3.8. The Dynamics of Fixed Assets Indices of Across the Federal Okrugs $(1996=100 \%)$

The existing investment statistics do not seriously improve the situation, as it is hard to bring the data to a comparable form (there is no reliable deflator on hand), while the building of the indicator on the basis of the constant inventory methods requires fairly long series.

The dynamics of fixed assets presented in Fig. 3.8 appear poorly correspondent to the fixed assets renovation and replacement statistics. According to the official data, during the period in question, the commissioning of fixed assets into operation by the economy as whole did not exceed $1.5 \%$ of their volume as of end-year, while their replacement rate did not exceed $1.3 \%$ of their volume as of the beginning of the year. The dynamics of the coefficients witness that the volume of fixed assets had remained practically constant prior to 1999, after which there arose a trend to their insignificant 
growth (with the renovation coefficients advancing the replacement ones). Underlying such discrepancies is likely to be the ongoing revaluations of fixed assets.

Due to the absence of the necessary information on the regional level, it appears impossible to directly employ the approach that is based on the renovation and replacement coefficients (the IET expert team (Entov, Lugovoy at al. (2003)) used this particular approach to decompose growth on the sectoral level).

That is why the index of the physical volume of fixed assets of $i-$ region was built basing on the data on investments. In the course of conduct of the assessment it is assumed that the regional structure of investment coincides with the regional structure of fixed assets commissioning, while their replacement rate is equal across all the regions:

$$
F A^{i}(t)=F A^{i}(t-1)+R n^{i}(t)-R p^{i}(t),
$$

where

$F A^{i}(t)$ - volume of fixed assets of $i$ region as of the end of period $t$;

$R n^{i}(t)$ - commissioning fixed assets of $i$ region in period $t$, computed on the basis of the data on investment and the fixed assets renovation rate across Russia as a whole $k_{t}^{R n}$ : $R n^{i}(t)=\frac{I^{i}(t)}{I(t)} k_{t}^{R n} F A(t)$,

$F A(t)$ - volume of fixed assets across Russia as a whole as of end-period $t$,

$I^{i}(t)$ - investments of $i$ region in period $t$,

$I(t)$ - investments across Russia as a whole in period $t$, 
$R p^{i}(t)$ - withdrawal of fixed assets of $i$ region in period $t$, computed on the basis of the fixed assets replacement rate across Russia as a whole $k_{t}^{R p}: R p^{i}(t)=k_{t}^{R p} F A^{i}(t-1)$.

Thus built index of the physical volume of fixed assets demonstrates loose positive dynamics for most of the federal okrugs between 1997 and 2002 (see Fig. 3.9), except for the Siberian and Far-Eastern ones. As concerns the Siberian okrug, the trend of its index is strictly negative.

In any case, the range of the change of assets accumulated over the six years in question was found to be within $5 \%$.

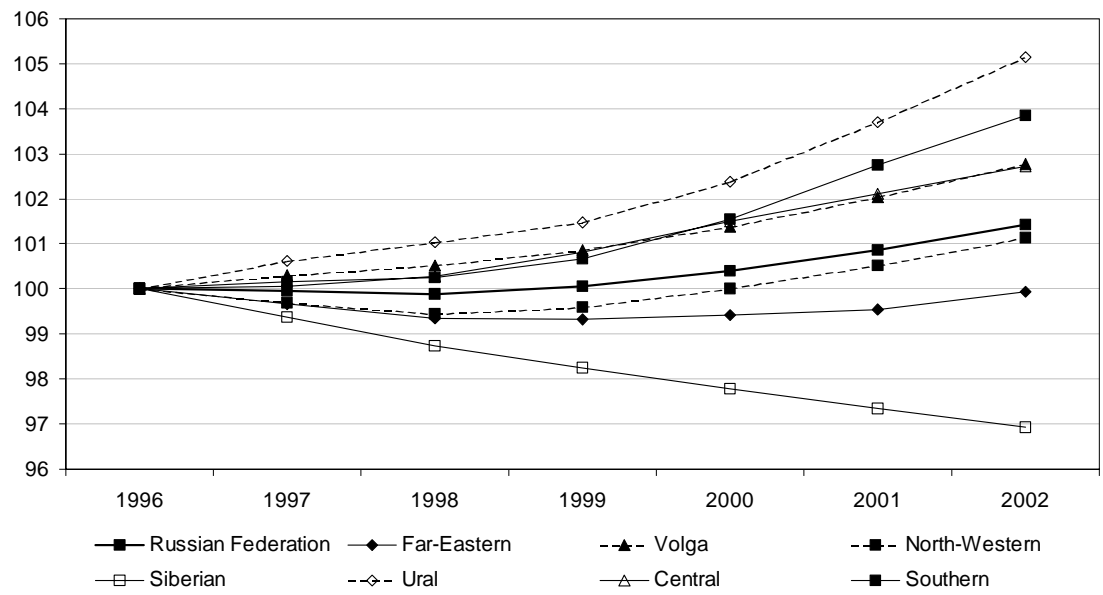

Fig. 3.9. The Index of the Physical Volume of Fixed Assets by Federal Okrugs $(1996=100 \%)$

Let us once again note that the index of the physical volume of FA received on the basis of the renovation and replacement rates forms rather a rough approximation of the dynamics of the physical volume of capital. It does not consider changes in the productive 
capacities' efficiency due to their age, physical depreciation and moral ageing. This leads to an additional error of measure of the contribution capital services make to economic growth, thus increasing an unexplained by production factors remainder (TFP). The theoretical assessment of capital input (in the assumption that technical progress is not embodied in capital) is built on the basis of the weighted sum of production means of different age (see (3.7)).

In addition, the FA replacement rate does not mirror the latent replacement of the production equipment due to a change in a given product range, decay and depreciation of production capacities that continue to remain on enterprises balance-sheets. The nationwide FA depreciation rate grew on average from $41.9 \%$ in 1999 up to $47.9 \%$ in 2002. An analogous situation is noted for most regions (see Annex 2, Table A2-7). The most substantial rise in the depreciation rate was noted in Kostroma oblast (from 37.4 to $55.3 \%$ ), Republic of Tyva (from 35.5 to $48.5 \%$ ), city of Moscow (37.7 to 50.1\%), Khabarovsk krai (32.7 to 44.7\%), Penza oblast (42.2 to 53.7\%). In 2002, the level of depreciation of FA in Kirov and Yroslavl oblasts exceeded 57\%. The depreciation of FA in 1999-2002 fell only in 12 regions: republics of Ingoushetia, kalmykia, Altay, Chuvashia, North Ossetia-Alania, Dagestan, Karachaevo-Cherkessia, Krasnodar krai, Tomsk, Astrakhan, Arkhangel'sk and Tyumen oblasts, and for most of them the fall was insignificant.

As it noted in the case of labor input, the dynamics of the index of the physical volume of FA likewise display no visible similarity to the dynamics of the GRP index (see Fig. 3.1). A considerable fall in GRP index is accompanied by almost constant value of the index of the physical volume of FA.

This can be attributed to the fact that the volume of production means does not fully mirror the capital formation process, as it overvalues the assessment of their part that is actually involved in 
production. Plus, the given series imply the constancy of output depending on the age of the equipment and improvement of means of production, which increases their net contribution to output.

A more accurate assessment of capital necessitates the account of a change in the volume of production capacities as well as intensity of their use. In the present paper, the intensity of the use of FA is computed basing on the data on the level of electricity consumption (such a method was employed by Griliches, Jorgenson (1967); Costello $(1993)^{32}$ ), whose dynamics across the federal okrugs are given in Fig. 3.10.

Whilst employing the electricity consumption indicator as an indicator of the capacity loading rate, one needs to conduct an adjustment at the change of the average capacity of the production equipment involved in the production process. There are no necessary statistical data to make the respective assessments by regions. However, as during the period in question FA renovation rate by the economy as a whole has not exceeded $1.5 \%$ of their volume as of end-year, it appears possible not to adjust the indicators.

The product of the multiplication of the index of the physical volume of FA by the capacity loading level index is regarded as the capital index (Fig. 3.11).

\footnotetext{
${ }^{32}$ According to Denison, the employment of the electricity consumption indicator as the one of production capacity loading is incorrect, primarily because there are no adjustments made to the economic cycle. Second, an accurate assessment necessitates the use of kilowatt-hours consumed by means of production for which electricity forms a major source of power.
} 


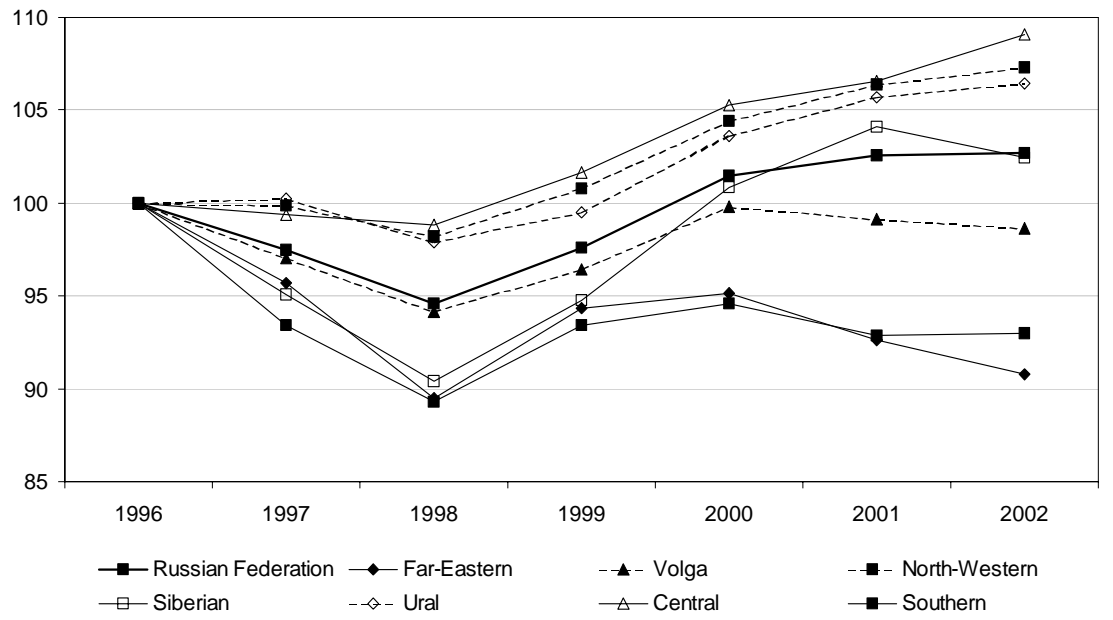

Fig. 3.10. Capacity Loading Rate Index by the Federal Okrugs $(1996=100 \%)$

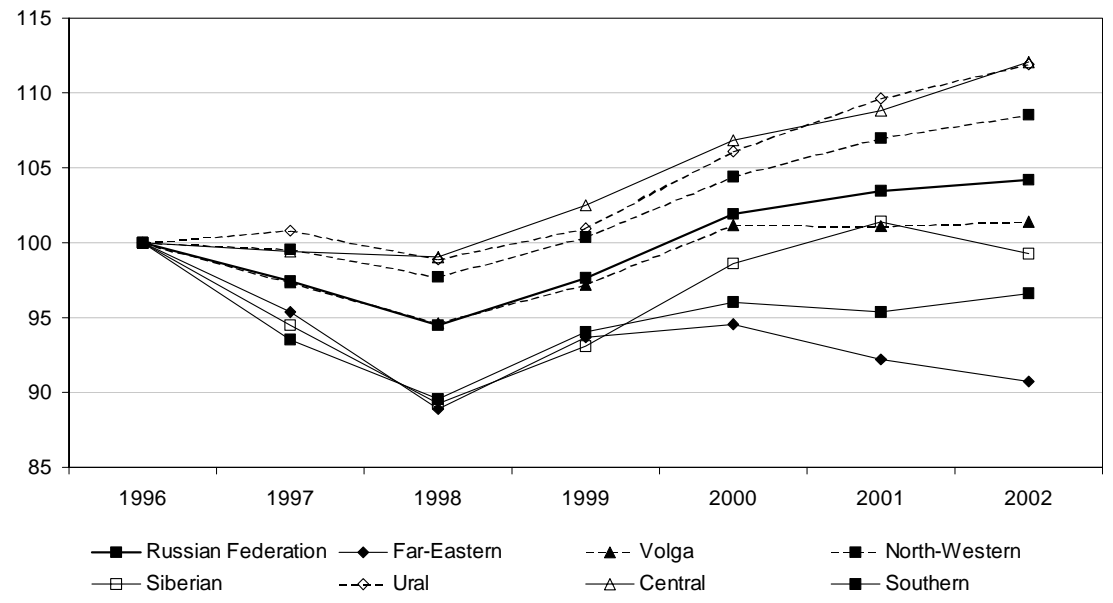

Fig. 3.11. Capital Index by the Federal Okrugs (1996 = 100\%) 
Table 3.4

\section{Results of Assessment of the Output Growth rates and Contribution of Components of Capital Input by the Federal Okrugs}

\begin{tabular}{|c|c|c|c|c|c|c|c|}
\hline \multirow[b]{2}{*}{ Federal okrug } & \multicolumn{4}{|c|}{ Growth rates } & \multicolumn{3}{|c|}{ As \% of the GRP growth rates } \\
\hline & $\begin{array}{c}\text { Effect from } \\
\text { the change in } \\
\text { the volume of } \\
\text { FA }\end{array}$ & $\begin{array}{l}\text { Effect from } \\
\text { the change of } \\
\text { the level of } \\
\text { capacity } \\
\text { loading }\end{array}$ & $\begin{array}{l}\text { Effect from } \\
\text { capital input }\end{array}$ & GRP & $\begin{array}{c}\text { Effect from } \\
\text { the change in } \\
\text { the volume of } \\
\text { FA }\end{array}$ & $\begin{array}{l}\text { Effect from } \\
\text { the change of } \\
\text { the level of } \\
\text { capacity } \\
\text { loading }\end{array}$ & $\begin{array}{l}\text { Effect from } \\
\text { capital input }\end{array}$ \\
\hline \multicolumn{8}{|c|}{ 1997-1998 } \\
\hline Far-Eastern & -0.09 & -1.52 & -1.61 & -4.80 & 1.83 & 31.66 & 33.49 \\
\hline Volga & 0.13 & -1.56 & -1.43 & -2.63 & -5.05 & 59.34 & 54.28 \\
\hline North-Western & -0.10 & -0.34 & -0.44 & -3.22 & 3.14 & 10.68 & 13.82 \\
\hline Siberian & -0.26 & -2.03 & -2.29 & -6.69 & 3.82 & 30.40 & 34.21 \\
\hline Ural & 0.27 & -0.54 & -0.27 & -2.92 & -9.17 & 18.44 & 9.27 \\
\hline Central & 0.08 & -0.32 & -0.25 & 0.12 & 62.46 & -265.28 & -202.82 \\
\hline Southern & 0.06 & -2.48 & -2.42 & -3.66 & -1.52 & 67.66 & 66.15 \\
\hline \multicolumn{8}{|c|}{ 1999-2002 } \\
\hline Far-Eastern & 0.05 & 0.17 & 0.22 & 4.60 & 0.98 & 3.71 & 4.70 \\
\hline Volga & 0.28 & 0.64 & 0.92 & 5.52 & 5.11 & 11.54 & 16.66 \\
\hline North-Western & 0.17 & 0.96 & 1.13 & 7.46 & 2.29 & 12.89 & 15.18 \\
\hline Siberian & -0.19 & 1.35 & 1.16 & 5.60 & -3.39 & 24.20 & 20.81 \\
\hline Ural & 0.53 & 1.13 & 1.66 & 6.22 & 8.53 & 18.19 & 26.72 \\
\hline Central & 0.35 & 1.45 & 1.80 & 7.72 & 4.54 & 18.78 & 23.32 \\
\hline Southern & 0.40 & 0.50 & 0.90 & 8.17 & 4.90 & 6.14 & 11.05 \\
\hline \multicolumn{8}{|c|}{ 1997-2002 } \\
\hline Far-Eastern & 0.00 & -0.40 & -0.40 & 1.37 & 0.06 & -28.99 & -28.96 \\
\hline Volga & 0.23 & -0.10 & 0.13 & 2.73 & 8.50 & -3.65 & 4.83 \\
\hline North-Western & 0.08 & 0.52 & 0.60 & 3.77 & 2.13 & 13.89 & 16.00 \\
\hline Siberian & -0.21 & 0.21 & 0.00 & 1.33 & -15.93 & 15.92 & -0.03 \\
\hline Ural & 0.44 & 0.57 & 1.01 & 3.08 & 14.36 & 18.56 & 32.89 \\
\hline Central & 0.26 & 0.86 & 1.11 & 5.13 & 5.05 & 16.70 & 21.73 \\
\hline Southern & 0.29 & -0.50 & -0.22 & 4.07 & 7.00 & -12.30 & -5.34 \\
\hline
\end{tabular}


The computations (see Table 3.4) testify that for most regions capital input form a more significant growth factor than labor input (see Table 3.3 and Table 3.4), which becomes especially notable in the period of growth of 1999-2002. Given the above, as it was noted in the case of labor input, the impact of capital inputon the output growth rate likewise appeared more substantial at the stage of decline (1997-1998) than at the stage of growth in output (1999-2002).

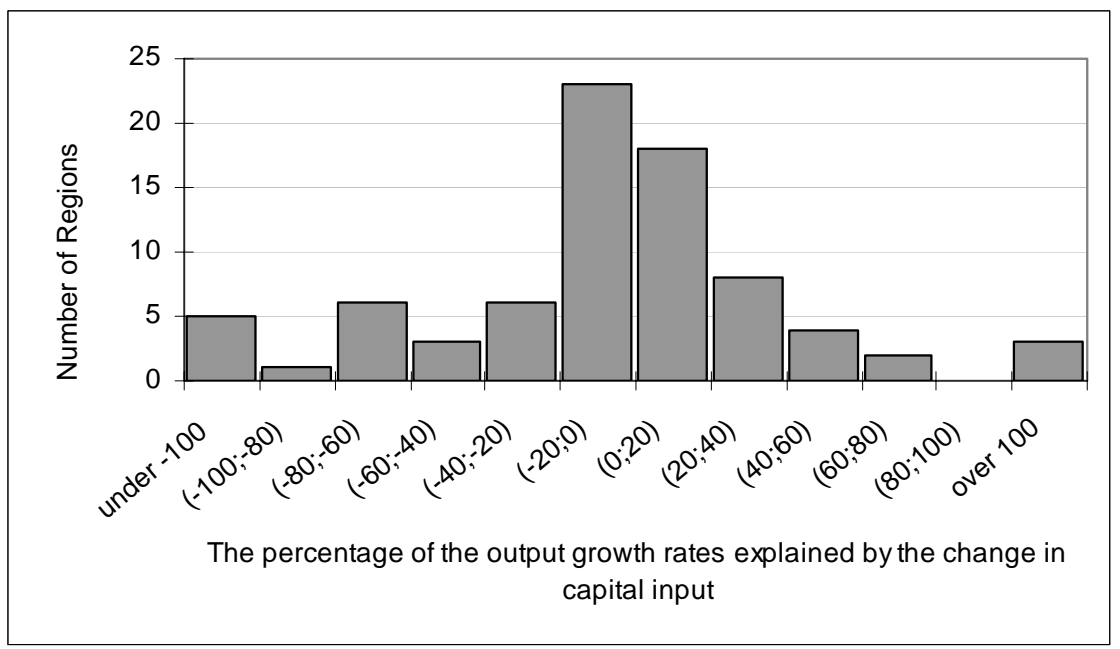

Fig. 3.12. Bar Chart of the Output Growth Rate Explained by Changes in Capital Input Across Regions (the Period between 1997 and 2002)

As it noted for the case of labor input, the percentage of output growth rates explained by changes in capital inputlikewise varies across regions. There exist a number of them for which the direction of changes in the output growth rates appears opposite to the one of changes in the capital inputgrowth rates (negative values in the Bar Chart). For most regions, changes in the volume of fixed assets in a region with account of their loading level explain 0-40\% 
of their GRP growth rates (see Fig. 3.12). Similar to the case of labor input, negative values of the proportion of the output growth rates explained by changes in capital input are largely explained by the selection of the initial and final points of a given time interval. In sub-periods (see Annex 2, Fig. A2-5 and A2-6), the indicator value for most of regions finds itself within 0-20\%. In 1997-1998, only $14 \%$ of regions saw changes in their output coupled by an opposite change in capital input, wile the proportion of such regions grew up to 36\% in 1999-2002.

\subsubsection{Assessing Weight Coefficients to Integrate Inputs}

As noted above, decomposition requires the presence of marginal factor products and their proportion in the output on hand to have a possibility for computing weight coefficients $\alpha_{\mathrm{L}}$ and $\alpha_{K}$ (see (3.2)-(3.4)) to integrate factor costs.

There exist two main methods to assess the coefficients: (1) econometric and (2) basing on proportions of factors in the output (in the assumption of the equality between marginal products of factors and prices for them). Econometric method entails some complexities $^{33}$ and cannot be employed in the absence of a sufficient mass of data. That is why in this paper we employed the other, widespread method.

In the assumption of the constant retutns to scale regarded as a TFP factor and that enterprises are keen to reduce their production costs and, consequently, use costs of various kinds in proportions

\footnotetext{
${ }^{33}$ While the econometric approach allows abandonment the assumption of the equality between marginal factor products and observed costs, thus obtained assessments may prove to be biased. First, the basic factors growth rates cannot always be regarded as exogenous towards changes in TFP; second if factors costs have been measured with errors, the standard methods of assessing coefficients of the equation would lead to inconsistent estimates. Barro, R., X. Sala-iMartin (2004). P. 433-457.
} 
that reduce the costs, different factors incomes are proportional to their marginal products. For each year of the period in question by Russia as a whole:

- the proportion of the remuneration of employees ${ }^{34}$ in GDP is regarded as the weight coefficient;

- the difference between unit and the weight coefficient of labor inputis regarded as the coefficient of capital input.

The data on GRP formation of of Russian regions by income sources have become available since $2002^{35}$, but regional computations of the employees' remuneration do not suggest considering and reflecting in the account a hidden compensation of employees. On the federal level, finding volumes of the hidden compensation of employees is conducted according to a methodology that suggests building all the accounts of the "Households" sector, which is not applicable to regional computations.

That is why while computing weight coefficients of labor input needed to conduct decomposition of regional growth, one suggests that the wages fund of the employed to employees' remuneration ratio is equal for all the regions and coincides with the analogous nationwide indicator.

As the table shows, the range of the coefficients is fairly great (from 0.38 to 0.75 ). This can be associated with quite serious interregional (and inter-okrug) differences in terms of economic structure. However, there can exist yet another reason, that is, the failure to meet preconditions laid down in the methodology, should they are not met to a various extent for different regions, this would also lead to a distortion in the course of the comparison of assessments, because of their bias.

\footnotetext{
${ }^{34}$ Source: "Natsionalnye scheta Rossii”, Rosstat RF.

${ }^{35}$ Source: “Natsionalnye scheta Rossii v 1996-2003 gg.”, Rosstat RF.
} 
Table 3.5

Weight Coefficients of Labor Input for the Federal Okrugs

\begin{tabular}{lccccccc}
\hline Federal okrug & $\mathbf{1 9 9 6}$ & $\mathbf{1 9 9 7}$ & $\mathbf{1 9 9 8}$ & $\mathbf{1 9 9 9}$ & $\mathbf{2 0 0 0}$ & $\mathbf{2 0 0 1}$ & $\mathbf{2 0 0 2}$ \\
\hline Far-Eastern & 0.75 & 0.74 & 0.69 & 0.61 & 0.66 & 0.69 & 0.72 \\
Volga & 0.48 & 0.49 & 0.48 & 0.45 & 0.46 & 0.51 & 0.54 \\
North-Western & 0.63 & 0.66 & 0.57 & 0.52 & 0.56 & 0.63 & 0.64 \\
Siberian & 0.60 & 0.60 & 0.58 & 0.55 & 0.58 & 0.62 & 0.65 \\
Ural & 0.46 & 0.49 & 0.49 & 0.45 & 0.44 & 0.49 & 0.49 \\
Central & 0.48 & 0.48 & 0.45 & 0.40 & 0.38 & 0.44 & 0.43 \\
Southern & 0.56 & 0.58 & 0.53 & 0.50 & 0.52 & 0.57 & 0.60 \\
$\begin{array}{l}\text { By RF as a } \\
\text { whole }\end{array}$ & 0.55 & 0.56 & 0.53 & 0.47 & 0.47 & 0.53 & 0.53 \\
\hline
\end{tabular}

An alternative method that partly lacks this particular drawback is the employment of interval estimates, or an expert pre-setting of weights, being uniform for all the subjects in question. Weight coefficients $\alpha_{K}=0,3$ и $\alpha_{L}=0,7$ are most frequently used (see for instance: De Broek, Koen (2000); Dolinskaya (2001); Entov, Lugovoy at al. (2003) $)^{36}$. However, in this particular case inter-regional and inter-okrug differences in terms of services prices of the basic factors would not be taken into account in the course of the TFP assessment, which can dramatically affect interpretation of the respective results.

The growing weight coefficient of labor (the share of employees' remunerationin GRP) appeared characteristic of most of the federal okrugs in 1997-2002. Its average growth rate accounted for $7-10 \%$ of the value as of the beginning of the period, despite a drop in the value in 1998-1999. The Central and Far-Eastern federal okrugs made an exception in this respect, for in the period in question their weight coefficients of labor input slid by $4.7 \%$ and $10.0 \%$,

\footnotetext{
${ }^{36}$ For reference: in Bessonov (2002), the estimates of factor elasticities for the Soviet economy make up EK = 0,45 и $\mathrm{EL}=0,55$.
} 
respectively. As concerns regions, only in 22 of them saw a drop in the coefficient values (see Annex 2, Table A2-5).

\subsubsection{Decomposition of Growth}

The final stage of decomposition comprises integration of growth rates of the basic factors computed on the basis of the earlier built indices by means of weight coefficients and computation of the unexplained remainder. Results of the decomposition are given in Table 3.6 (see also Annex 2, Map 1).

Table 3.6

\section{Decomposition of Growth in GRP by the Federal Okrugs for the Period between 1997 and 2002}

\begin{tabular}{lccccccc}
\hline & $\begin{array}{c}\text { Far- } \\
\text { Eastern }\end{array}$ & Volga & $\begin{array}{c}\text { North- } \\
\text { Western }\end{array}$ & Siberian & Ural & Central & $\begin{array}{c}\text { South- } \\
\text { ern }\end{array}$ \\
\hline GRP & 1.37 & 2.73 & 3.77 & 1.33 & 3.08 & 5.13 & 4.07 \\
$\begin{array}{l}\text { I. Factor in- } \\
\text { puts }\end{array}$ & -0.58 & 0.33 & 0.61 & -0.16 & 1.13 & 1.33 & 0.16 \\
$\begin{array}{l}\text { I.1 Labor } \\
\text { (Employment) }\end{array}$ & -0.19 & 0.20 & 0.00 & -0.16 & 0.11 & 0.22 & 0.38 \\
$\quad \begin{array}{l}\text { I.2 Capital } \\
\quad \text { Fixed assets }\end{array}$ & -0.40 & 0.13 & 0.60 & 0.00 & 1.01 & 1.11 & -0.22 \\
$\quad \begin{array}{l}\text { Loading } \\
\text { rate }\end{array}$ & -0.40 & -0.10 & 0.52 & 0.21 & 0.57 & 0.86 & -0.50 \\
$\begin{array}{l}\text { Employment } \\
\text { structure }\end{array}$ & -0.39 & -0.23 & 0.04 & -0.41 & 0.10 & -0.27 & -0.52 \\
$\begin{array}{l}\text { II. Remainder } \\
\text { The Solow }\end{array}$ & 2.34 & 2.63 & 3.13 & 1.90 & 1.86 & 4.06 & 4.44 \\
remainder & 1.60 & 2.33 & 3.73 & 1.75 & 2.55 & 4.66 & 3.47 \\
\hline
\end{tabular}

The period between 1997 and 2002 saw positive TFP growth rates for all the federal okrugs. In addition, this remainder forms the most significant factor of growth - for all the federal okrugs, the proportion of the output growth rates generated by the growth in the remainder have been over $60 \%$, while in the Far-Eastern, Siberian and Southern federal okrugs, positive growth rates of the 
remainder have practically compensated for a substantial fall in te basic factor costs.

Results of computations on the sub-periods 1997-98 and 19992002 (see Annex 2, Tables A2-9, A2-10) evidence that the growth rates of the unexplained remainder in the Volga, Central and Southern federal okrugs are positive both at the stage of growth and decline, while in other federal okrugs the TFP growth rates are positive only in 1999-2002. Given the above, at the stage of decline the fall in output for most federal okrugs (except for the Ural and Central ones) is largely explained by a fall in the basic factor costs, while at the stage of growth, for all the federal okrugs roughly $75 \%$ of the output growth rates explain the remainder growth rates.

The percentage of the output growth rates unexplained by changes in the basic factors vary substantially by regions (see $A n-$ nex 2, Table A2-11). For most of them, the remainder growth rates over the period in question coincide with those of output. Interestingly, the proportion of the output growth rates explained by the basic factors has not exceeded the proportion they have failed to explain.

The diagram of dispersion of the output growth rates and those of different factors (see Annex 2, Fig. A2-7) exposes a fairly loose connection between the GRP growth rates and those of labor and capital. A small percentage of the explained growth manifests itself in the structure of TFP assessments that practically repeats the structure of GRP (see Fig. 3.13). This is explained by fairly approximate estimates of factors, on the one hand, and the selected assessment interval that covers both the output growth and decline trends, on the other. 


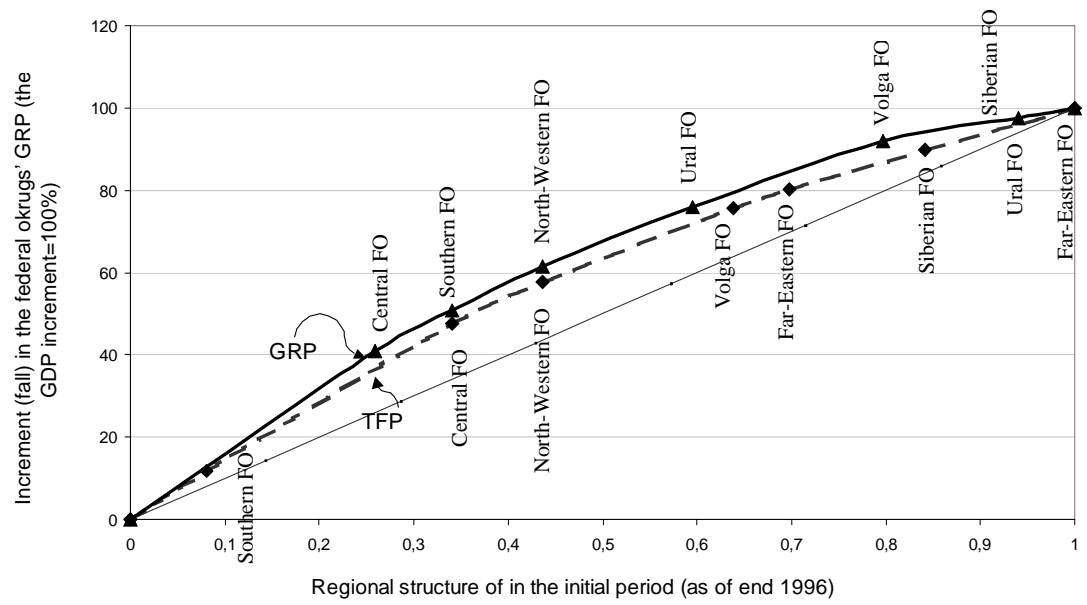

Fig. 3.13. Sun-Rise Diagram of growth in GDP (1997-2002) at the Federal Okrug Level

As noted above, the regions display the non-homogenous dynamics of output, with the differences being determined both by growth rates and the moment of the change of the trend (Fig. 3.2). That is why let us consider results of decomposition of the regions' growth rates by factors individually for the periods of decline and growth (the regions have been broken down into groups, depending on the limits of the periods). The first group comprises regions whose dynamics of output demonstrated growth over the whole period of 1997-2002, as well as those whose output volume has never slid below the 1996 level.

For all the regions of the group except Moscow, the unexplained remainder exceeds the contribution of basic factors. 
Table 3.7

Decomposition of the Regions' Output Growth Rates in1997-2002

\begin{tabular}{|c|c|c|c|c|c|c|c|c|}
\hline & \multirow[b]{2}{*}{ GRP } & \multirow[b]{2}{*}{$\begin{array}{c}\text { I. } \\
\text { Factor } \\
\text { inputs }\end{array}$} & \multirow{2}{*}{$\begin{array}{c}\text { I.1. } \\
\text { Labor } \\
\text { employ } \\
\text { ment) }\end{array}$} & \multirow[b]{2}{*}{$\begin{array}{c}\text { I.2. } \\
\text { Capi- } \\
\text { tal }\end{array}$} & \multicolumn{2}{|c|}{ of which } & \multirow{2}{*}{$\begin{array}{c}\text { Employ- } \\
\text { ment } \\
\text { struc- } \\
\text { ture }\end{array}$} & \multirow[b]{2}{*}{$\begin{array}{l}\text { II. Re- } \\
\text { mainder }\end{array}$} \\
\hline & & & & & $\begin{array}{l}\text { Fixed } \\
\text { assets }\end{array}$ & $\begin{array}{l}\text { Load- } \\
\text { ing } \\
\text { rate }\end{array}$ & & \\
\hline $\begin{array}{l}\text { Astrakhan } \\
\text { oblast }\end{array}$ & 7.05 & 0.55 & 0.00 & 0.55 & 0.25 & 0.30 & -0.96 & 7.45 \\
\hline $\begin{array}{l}\text { Belgorod } \\
\text { oblast }\end{array}$ & 5.12 & 1.33 & 0.37 & 0.95 & 0.09 & 0.86 & -0.40 & 4.19 \\
\hline $\begin{array}{l}\text { City of Mos- } \\
\text { cow }\end{array}$ & 6.69 & 3.58 & 0.40 & 3.18 & 1.06 & 2.12 & -0.09 & 3.20 \\
\hline Kursk oblast & 3.50 & 0.42 & 0.72 & -0.30 & -0.19 & -0.11 & -0.59 & 3.67 \\
\hline $\begin{array}{l}\text { Moscow } \\
\text { oblast }\end{array}$ & 4.79 & 0.83 & 0.49 & 0.34 & 0.34 & - & -0.14 & 4.10 \\
\hline $\begin{array}{l}\text { Murmansk } \\
\text { oblast }\end{array}$ & 1.70 & -0.68 & -0.36 & -0.32 & -0.12 & -0.20 & -0.52 & 2.90 \\
\hline Orel oblast & 6.07 & 0.30 & 0.68 & -0.38 & -0.06 & -0.32 & -0.41 & 6.18 \\
\hline Perm oblast & 3.87 & 0.43 & 0.36 & 0.06 & 0.06 & - & -0.04 & 3.48 \\
\hline $\begin{array}{l}\text { Republic of } \\
\text { Mordovia }\end{array}$ & 4.39 & 0.07 & 0.09 & -0.03 & -0.05 & 0.03 & -0.17 & 4.49 \\
\hline Tambov oblast & 5.43 & -1.25 & -0.20 & -1.05 & -0.24 & -0.81 & -0.58 & 7.26 \\
\hline
\end{tabular}

Table 3.8

Decomposition of Growth in GRP by the Federal Okrugs in 1997 and 1998-2002

\begin{tabular}{|c|c|c|c|c|c|c|c|c|}
\hline & \multirow[b]{2}{*}{ GRP } & \multirow{2}{*}{$\begin{array}{l}\text { I. } \\
\text { Factor } \\
\text { inputs }\end{array}$} & \multirow{2}{*}{$\begin{array}{c}\text { I.1. } \\
\text { Labor } \\
\text { employ } \\
\text { ment) }\end{array}$} & \multirow{2}{*}{$\begin{array}{c}\text { I.2. } \\
\text { Capi- } \\
\text { tal }\end{array}$} & \multicolumn{2}{|c|}{ of which } & \multirow{2}{*}{$\begin{array}{c}\text { Employ- } \\
\text { ment } \\
\text { struc- } \\
\text { ture }\end{array}$} & \multirow[b]{2}{*}{$\begin{array}{l}\text { II. Re- } \\
\text { mainder }\end{array}$} \\
\hline & & & & & $\begin{array}{l}\text { Fixed } \\
\text { assets }\end{array}$ & $\begin{array}{l}\text { Load- } \\
\text { ing } \\
\text { rate }\end{array}$ & & \\
\hline \multirow{2}{*}{$\begin{array}{l}\text { Republic of } \\
\text { Kabardino- } \\
\text { Balkaria }\end{array}$} & -2.43 & -5.96 & -1.49 & -4.47 & -0.07 & -4.40 & -0.02 & 3.56 \\
\hline & 9.02 & 1.07 & 1.87 & -0.80 & 0.12 & -0.92 & -0.56 & 8.51 \\
\hline \multirow{2}{*}{$\begin{array}{l}\text { Novgorod } \\
\text { oblast }\end{array}$} & -2.94 & -7.24 & -5.43 & -1.80 & -0.14 & -1.66 & 1.17 & 3.13 \\
\hline & 4.88 & 0.02 & 0.23 & -0.20 & 0.11 & -0.32 & 0.07 & 4.78 \\
\hline \multirow{2}{*}{$\begin{array}{l}\text { Republic of } \\
\text { North Ossetia- } \\
\text { Alania }\end{array}$} & -5.55 & -0.44 & -0.35 & -0.09 & -0.09 & - & -1.18 & -3.93 \\
\hline & 9.32 & 1.72 & 1.73 & -0.01 & -0.01 & - & -0.71 & 8.30 \\
\hline \multirow{2}{*}{ Tver oblast } & -8.66 & -0.09 & -0.83 & 0.74 & -0.17 & 0.91 & 0.03 & -8.61 \\
\hline & 3.54 & -0.50 & -0.04 & -0.46 & 0.09 & -0.55 & -0.41 & 4.45 \\
\hline
\end{tabular}


Table 3.9

Decomposition of Growth in the Regions' Output in 1997-1998 and 1999-2002

\begin{tabular}{|c|c|c|c|c|c|c|c|c|}
\hline & \multirow[b]{2}{*}{ GRP } & \multirow{2}{*}{$\begin{array}{l}\text { I. } \\
\text { Factor } \\
\text { inputs }\end{array}$} & \multirow{2}{*}{$\begin{array}{c}\text { I.1. } \\
\text { Labor } \\
\text { emplo } \\
\text { y- } \\
\text { ment) }\end{array}$} & \multirow{2}{*}{$\begin{array}{c}\text { I.2. } \\
\text { Capi- } \\
\text { tal }\end{array}$} & \multicolumn{2}{|c|}{ of which } & \multirow{2}{*}{$\begin{array}{c}\text { Employ- } \\
\text { ment } \\
\text { struc- } \\
\text { ture }\end{array}$} & \multirow{2}{*}{$\begin{array}{c}\text { II. Re- } \\
\text { main- } \\
\text { der }\end{array}$} \\
\hline & & & & & $\begin{array}{l}\text { Fixed } \\
\text { assets }\end{array}$ & $\begin{array}{l}\text { Load- } \\
\text { ing rate }\end{array}$ & & \\
\hline 1 & 2 & 3 & 4 & 5 & 6 & 7 & 8 & 9 \\
\hline \multirow{2}{*}{ Altay krai } & -9.52 & -3.28 & -1.28 & -1.90 & -0.22 & -1.68 & 0.00 & -6.23 \\
\hline & 6.10 & -0.75 & 0.68 & -1.45 & -0.15 & -1.30 & -0.64 & 7.49 \\
\hline \multirow{2}{*}{$\begin{array}{l}\text { Amur } \\
\text { oblast }\end{array}$} & -10.27 & -4.46 & -2.08 & -2.44 & -0.17 & -2.27 & -1.33 & -4.47 \\
\hline & 5.44 & 0.81 & 0.33 & 0.48 & -0.05 & 0.52 & -0.36 & 5.00 \\
\hline \multirow{2}{*}{$\begin{array}{l}\text { Ark- } \\
\text { hangel'sk } \\
\text { oblast }\end{array}$} & -3.87 & -3.98 & -3.18 & -0.88 & -0.21 & -0.67 & 0.00 & 0.10 \\
\hline & 8.18 & 1.87 & 1.22 & 0.64 & 0.03 & 0.61 & -0.37 & 6.68 \\
\hline \multirow{2}{*}{$\begin{array}{l}\text { Vladimir } \\
\text { oblast }\end{array}$} & -2.37 & -0.76 & -0.11 & -0.64 & -0.12 & -0.53 & -0.72 & -0.89 \\
\hline & 5.97 & 1.60 & 0.49 & 1.11 & -0.04 & 1.16 & 0.01 & 4.35 \\
\hline \multirow{2}{*}{$\begin{array}{l}\text { Volgograd } \\
\text { oblast }\end{array}$} & -5.30 & -9.43 & -3.43 & -5.91 & -0.15 & -5.76 & -0.37 & 4.50 \\
\hline & 6.38 & 1.24 & 1.48 & -0.42 & -0.07 & -0.34 & -1.04 & 6.18 \\
\hline \multirow{2}{*}{$\begin{array}{l}\text { Vologda } \\
\text { oblast }\end{array}$} & -2.62 & -2.49 & -2.45 & -0.07 & -0.01 & -0.06 & 0.67 & -0.80 \\
\hline & 6.08 & 2.18 & 1.61 & 0.57 & 0.01 & 0.56 & -0.35 & 4.24 \\
\hline \multirow{2}{*}{$\begin{array}{l}\text { Voronezh } \\
\text { oblast }\end{array}$} & -1.87 & -3.31 & -1.38 & -1.89 & -0.26 & -1.64 & -0.43 & 1.87 \\
\hline & 4.25 & 1.41 & 1.28 & 0.10 & -0.12 & 0.22 & -0.50 & 3.34 \\
\hline \multirow{2}{*}{$\begin{array}{l}\text { City of St. } \\
\text { Petersburg }\end{array}$} & -3.45 & -0.01 & -0.02 & 0.00 & -0.05 & 0.05 & 0.62 & -4.06 \\
\hline & 9.08 & 2.57 & 0.31 & 2.26 & 0.31 & 1.96 & 0.06 & 6.45 \\
\hline \multirow{2}{*}{$\begin{array}{l}\text { Jewish } \\
\text { autonomous } \\
\text { oblast }\end{array}$} & -14.45 & -5.54 & -4.84 & -0.74 & -0.10 & -0.64 & 0.07 & -8.98 \\
\hline & 6.17 & 2.50 & 2.27 & 0.15 & -0.13 & 0.28 & -1.45 & 5.12 \\
\hline \multirow{2}{*}{$\begin{array}{l}\text { Ivanovo } \\
\text { oblast }\end{array}$} & -9.44 & -3.33 & -2.53 & -0.71 & -0.22 & -0.49 & 0.22 & -6.34 \\
\hline & 4.93 & 0.70 & 0.10 & 0.61 & -0.17 & 0.78 & -0.48 & 4.71 \\
\hline \multirow{2}{*}{$\begin{array}{l}\text { Irkutsk } \\
\text { oblast }\end{array}$} & -10.71 & -2.11 & -1.13 & -1.03 & -0.23 & -0.79 & -0.78 & -7.82 \\
\hline & 2.54 & 1.60 & 0.67 & 0.91 & -0.17 & 1.08 & -0.51 & 1.45 \\
\hline \multirow{2}{*}{$\begin{array}{l}\text { Kaliningrad } \\
\text { oblast }\end{array}$} & -7.33 & -0.34 & 0.13 & -0.45 & -0.16 & -0.29 & -0.25 & -6.74 \\
\hline & 8.22 & 1.04 & 0.42 & 0.61 & 0.00 & 0.61 & -0.51 & 7.69 \\
\hline \multirow{2}{*}{$\begin{array}{l}\text { Kaluga } \\
\text { oblast }\end{array}$} & -5.88 & -2.71 & -2.12 & -0.59 & -0.10 & -0.50 & 0.48 & -3.65 \\
\hline & 4.73 & 1.32 & 0.29 & 0.97 & 0.03 & 0.94 & -0.67 & 4.08 \\
\hline
\end{tabular}




\begin{tabular}{|c|c|c|c|c|c|c|c|c|}
\hline 1 & 2 & 3 & 4 & 5 & 6 & 7 & 8 & 9 \\
\hline \multirow{2}{*}{$\begin{array}{l}\text { Kamchatka } \\
\text { oblast }\end{array}$} & -5.43 & -3.49 & -2.32 & -1.16 & -0.24 & -0.92 & 0.02 & -1.96 \\
\hline & -2.38 & -1.48 & -0.06 & -1.35 & -0.14 & -1.21 & -0.46 & -0.43 \\
\hline \multirow{2}{*}{$\begin{array}{l}\text { Kemerovo } \\
\text { oblast }\end{array}$} & -6.19 & -3.12 & -2.27 & -0.92 & 0.10 & -1.03 & -0.20 & -2.87 \\
\hline & 5.68 & 0.70 & 0.01 & 0.69 & 0.05 & 0.64 & -0.30 & 5.27 \\
\hline \multirow{2}{*}{$\begin{array}{l}\text { Kostroma } \\
\text { oblast }\end{array}$} & -1.98 & -1.74 & -1.66 & -0.07 & -0.14 & 0.08 & -0.11 & -0.13 \\
\hline & 3.81 & -0.26 & 0.15 & -0.43 & 0.07 & -0.50 & -0.18 & 4.25 \\
\hline \multirow{2}{*}{$\begin{array}{l}\text { Krasnodar } \\
\text { krai }\end{array}$} & -5.71 & -1.48 & -1.18 & -0.32 & 0.06 & -0.39 & -0.02 & -4.21 \\
\hline & 7.76 & 3.35 & 1.49 & 1.92 & 0.71 & 1.21 & -0.35 & 4.76 \\
\hline \multirow{2}{*}{$\begin{array}{l}\text { Kras- } \\
\text { noyarsk } \\
\text { krai }\end{array}$} & -3.27 & -1.06 & -0.77 & -0.29 & -0.06 & -0.22 & -0.40 & -1.81 \\
\hline & 4.99 & 0.55 & 0.61 & -0.06 & 0.01 & -0.07 & -0.56 & 5.00 \\
\hline \multirow{2}{*}{$\begin{array}{l}\text { Kurgan } \\
\text { oblast }\end{array}$} & -2.52 & -4.15 & -2.98 & -1.38 & -0.20 & -1.18 & 0.24 & 1.39 \\
\hline & 3.10 & 0.41 & 0.95 & -0.58 & -0.16 & -0.43 & -1.05 & 3.74 \\
\hline \multirow{2}{*}{$\begin{array}{l}\text { Leningrad } \\
\text { oblast }\end{array}$} & -4.74 & -0.85 & -0.83 & -0.04 & -0.04 & - & 0.27 & -4.17 \\
\hline & 12.01 & 1.14 & 0.66 & 0.48 & 0.48 & - & 0.17 & 10.70 \\
\hline \multirow{2}{*}{$\begin{array}{l}\text { Lipetsk } \\
\text { oblast }\end{array}$} & -5.03 & -5.66 & -1.37 & -4.30 & -0.17 & -4.14 & 0.03 & 0.61 \\
\hline & 6.07 & 1.76 & 1.25 & 0.34 & -0.10 & 0.44 & -0.44 & 4.75 \\
\hline \multirow{2}{*}{$\begin{array}{l}\text { Nizhny } \\
\text { Novgorod } \\
\text { oblast }\end{array}$} & -1.27 & -2.88 & -1.30 & -1.82 & -0.01 & -1.82 & 0.45 & 1.16 \\
\hline & 6.56 & 1.06 & 0.42 & 0.48 & 0.00 & 0.48 & 0.00 & 5.51 \\
\hline \multirow{2}{*}{$\begin{array}{l}\text { Novosibirsk } \\
\text { oblast }\end{array}$} & -5.45 & -5.26 & -2.21 & -3.07 & -0.16 & -2.91 & -0.17 & -0.01 \\
\hline & 8.31 & 1.18 & 0.88 & 0.25 & -0.09 & 0.34 & -0.37 & 7.50 \\
\hline \multirow{2}{*}{$\begin{array}{l}\text { Omsk } \\
\text { oblast }\end{array}$} & -8.81 & -3.17 & -0.41 & -2.88 & -0.19 & -2.69 & -0.54 & -5.09 \\
\hline & 6.92 & 0.04 & 0.47 & -0.43 & -0.13 & -0.30 & 0.58 & 6.30 \\
\hline \multirow{2}{*}{$\begin{array}{l}\text { Orenburg } \\
\text { oblast }\end{array}$} & -5.14 & -1.67 & -0.82 & -0.89 & -0.05 & -0.83 & -0.37 & -3.10 \\
\hline & 5.89 & 2.32 & 1.33 & 0.99 & 0.04 & 0.95 & -0.41 & 3.97 \\
\hline \multirow{2}{*}{$\begin{array}{l}\text { Penza } \\
\text { oblast }\end{array}$} & -5.76 & -3.11 & -1.67 & -1.47 & -0.16 & -1.30 & -0.13 & -2.53 \\
\hline & 5.99 & -0.16 & 1.12 & -1.29 & -0.09 & -1.20 & -0.76 & 6.91 \\
\hline \multirow{2}{*}{$\begin{array}{l}\text { Pskov } \\
\text { oblast }\end{array}$} & -9.87 & -3.80 & -2.15 & -1.65 & -0.22 & -1.42 & 0.13 & -6.20 \\
\hline & 6.62 & 1.16 & 1.58 & -0.43 & -0.14 & -0.30 & -0.81 & 6.28 \\
\hline \multirow{2}{*}{$\begin{array}{l}\text { Republic of } \\
\text { Adygea }\end{array}$} & -6.60 & -5.16 & -2.50 & -2.63 & -0.22 & -2.42 & -1.83 & 0.40 \\
\hline & 3.17 & 1.15 & 0.58 & 0.55 & -0.03 & 0.58 & -0.31 & 2.32 \\
\hline \multirow{2}{*}{$\begin{array}{l}\text { Republic of } \\
\text { Bashkor- } \\
\text { tostan }\end{array}$} & -4.27 & -3.66 & -0.83 & -2.84 & 0.09 & -2.92 & 0.15 & -0.75 \\
\hline & 5.04 & 1.00 & 0.62 & 0.34 & 0.26 & 0.08 & -0.49 & 4.53 \\
\hline
\end{tabular}




\begin{tabular}{|c|c|c|c|c|c|c|c|c|}
\hline 1 & 2 & 3 & 4 & 5 & 6 & 7 & 8 & 9 \\
\hline \multirow{2}{*}{$\begin{array}{l}\text { Republic of } \\
\text { Buryatiya }\end{array}$} & -2.04 & -8.05 & -5.30 & -2.79 & -0.12 & -2.67 & -0.36 & 6.37 \\
\hline & 6.19 & 1.26 & 0.98 & 0.23 & -0.07 & 0.30 & -1.04 & 5.97 \\
\hline \multirow{2}{*}{$\begin{array}{l}\text { Republic of } \\
\text { Dagestan }\end{array}$} & -3.89 & 3.42 & 2.12 & 1.27 & 0.32 & 0.95 & -0.58 & -6.73 \\
\hline & 10.76 & 1.87 & 1.59 & -0.57 & 0.14 & -0.72 & -1.57 & 10.47 \\
\hline \multirow{2}{*}{$\begin{array}{l}\text { Republic of } \\
\text { Karelia }\end{array}$} & -6.73 & -5.62 & -5.46 & -0.18 & -0.19 & 0.01 & -0.79 & -0.31 \\
\hline & 7.04 & 2.72 & 1.48 & 1.25 & -0.01 & 1.26 & -0.02 & 4.34 \\
\hline \multirow{2}{*}{$\begin{array}{l}\text { Republic of } \\
\text { Sakha (Ya- } \\
\text { kutia) }\end{array}$} & -4.93 & -3.75 & -3.33 & -0.45 & 0.08 & -0.53 & 0.10 & -1.29 \\
\hline & 4.13 & -0.26 & -0.18 & -0.11 & 0.36 & -0.47 & -0.61 & 5.01 \\
\hline \multirow{2}{*}{$\begin{array}{l}\text { Republic of } \\
\text { Tatarstan }\end{array}$} & -3.90 & -3.31 & -0.81 & -2.47 & 0.08 & -2.55 & -0.08 & -0.51 \\
\hline & 6.94 & 2.59 & 0.69 & 1.88 & 0.41 & 1.47 & -0.14 & 4.49 \\
\hline \multirow{2}{*}{$\begin{array}{l}\text { Republic of } \\
\text { Tyva }\end{array}$} & -2.41 & -3.88 & -3.58 & -0.29 & -0.29 & - & -0.28 & 1.75 \\
\hline & 6.45 & -1.12 & -0.89 & -0.23 & -0.23 & - & -0.31 & 7.88 \\
\hline \multirow{2}{*}{$\begin{array}{l}\text { Rostov } \\
\text { oblast }\end{array}$} & -1.06 & -5.48 & -1.75 & -3.78 & -0.15 & -3.63 & -1.04 & 5.46 \\
\hline & 9.62 & 2.58 & 1.07 & 1.52 & 0.07 & 1.46 & -0.41 & 7.45 \\
\hline \multirow{2}{*}{$\begin{array}{l}\text { Ryazan } \\
\text { oblast }\end{array}$} & -1.80 & -2.68 & -1.87 & -0.89 & -0.25 & -0.64 & -0.16 & 1.04 \\
\hline & 4.60 & 0.94 & 0.84 & 0.06 & -0.02 & 0.08 & -0.38 & 4.04 \\
\hline \multirow{2}{*}{$\begin{array}{l}\text { Samara } \\
\text { oblast }\end{array}$} & -1.48 & -2.00 & -0.66 & -1.26 & 0.03 & -1.29 & -0.41 & 0.92 \\
\hline & 4.71 & 2.08 & 0.82 & 1.25 & 0.15 & 1.10 & -0.18 & 2.81 \\
\hline \multirow{2}{*}{$\begin{array}{l}\text { Saratov } \\
\text { oblast }\end{array}$} & -1.52 & -1.84 & -0.60 & -1.34 & -0.11 & -1.23 & -0.41 & 0.73 \\
\hline & 6.60 & 0.31 & 0.34 & -0.03 & -0.01 & -0.02 & -0.60 & 6.89 \\
\hline \multirow{2}{*}{$\begin{array}{l}\text { Sakhalin } \\
\text { oblast }\end{array}$} & -2.56 & -4.34 & -2.66 & -1.68 & -0.01 & -1.67 & -1.37 & 3.16 \\
\hline & 4.66 & -0.36 & 0.86 & -1.34 & 0.53 & -1.87 & 0.02 & 5.00 \\
\hline \multirow{2}{*}{$\begin{array}{l}\text { Sverdlovsk } \\
\text { oblast }\end{array}$} & -5.78 & -1.64 & -1.06 & -0.57 & 0.07 & -0.64 & 0.14 & -4.28 \\
\hline & 6.37 & 1.94 & 0.73 & 1.23 & 0.06 & 1.17 & -0.34 & 4.77 \\
\hline \multirow{2}{*}{$\begin{array}{l}\text { Smolensk } \\
\text { oblast }\end{array}$} & -4.57 & -5.69 & -1.51 & -4.15 & -0.34 & -3.81 & -0.41 & 1.53 \\
\hline & 8.09 & 2.04 & 0.99 & 1.07 & 0.04 & 1.03 & -0.23 & 6.29 \\
\hline \multirow{2}{*}{$\begin{array}{l}\text { Stavropol } \\
\text { krai }\end{array}$} & -4.82 & -3.07 & -1.25 & -1.87 & -0.20 & -1.67 & -0.50 & -1.25 \\
\hline & 6.54 & 1.58 & 0.74 & 0.70 & 0.04 & 0.65 & -0.37 & 5.33 \\
\hline \multirow{2}{*}{$\begin{array}{l}\text { Tomsk } \\
\text { oblast }\end{array}$} & -6.63 & -3.27 & -2.02 & -1.28 & 0.00 & -1.28 & -0.10 & -3.27 \\
\hline & 8.12 & 1.86 & 0.70 & 1.16 & 0.13 & 1.03 & -0.01 & 6.27 \\
\hline \multirow{2}{*}{$\begin{array}{l}\text { Tyumen } \\
\text { oblast }\end{array}$} & -0.89 & -1.32 & -1.43 & 0.13 & 0.35 & -0.22 & 0.60 & -0.17 \\
\hline & 6.36 & 4.48 & 0.91 & 3.51 & 0.75 & 2.77 & -0.11 & 1.99 \\
\hline
\end{tabular}




\begin{tabular}{lcccccccc}
\hline \multicolumn{1}{c}{1} & 2 & 3 & 4 & 5 & 6 & 7 & 8 & 9 \\
\hline Republic of & -2.19 & -2.02 & -1.20 & -0.77 & -0.02 & -0.75 & -0.09 & -0.07 \\
Udmurtia & 4.48 & 2.66 & 1.36 & 1.33 & 0.09 & 1.24 & -0.34 & 2.17 \\
Ulyanovsk & -3.44 & -3.30 & -1.54 & -1.70 & -0.25 & -1.45 & -0.20 & 0.06 \\
oblast & 4.30 & -0.60 & 0.40 & -0.99 & -0.18 & -0.81 & -0.39 & 5.29 \\
Khabarovsk & -1.20 & -0.16 & -0.03 & -0.10 & -0.10 & - & -1.13 & 0.09 \\
krai & 8.45 & 1.62 & 1.48 & 0.14 & 0.14 & - & -0.17 & 7.00 \\
Chelyab- & -8.02 & -1.13 & -0.36 & -0.63 & -0.07 & -0.56 & -0.22 & -6.66 \\
insk oblast & 5.45 & -1.42 & 0.54 & -1.97 & 0.06 & -2.03 & -0.25 & 7.13 \\
Chita oblast & -11.14 & -4.02 & -3.87 & -0.14 & -0.14 & - & -0.86 & -6.26 \\
& 6.72 & 1.49 & 1.53 & -0.03 & -0.03 & - & -1.10 & 6.33 \\
Republic of & -7.36 & -1.41 & -1.11 & -0.28 & -0.15 & -0.13 & -0.13 & -5.83 \\
Chuvashia & 3.87 & 2.58 & 1.36 & 1.18 & -0.05 & 1.23 & -0.62 & 1.91 \\
Yaroslavl & -2.06 & -1.70 & -0.93 & -0.68 & -0.22 & -0.46 & -0.27 & -0.09 \\
oblast & 7.85 & 2.86 & 0.78 & 2.09 & 0.08 & 2.01 & -0.20 & 5.19 \\
\hline
\end{tabular}

Table 3.10

Decomposition of Growth in the Regions' Output in 1997-1999 and 2000-2002

\begin{tabular}{|c|c|c|c|c|c|c|c|c|}
\hline & \multirow[b]{2}{*}{ GRP } & \multirow[b]{2}{*}{$\begin{array}{c}\text { I. Fac- } \\
\text { tor } \\
\text { inputs }\end{array}$} & \multirow{2}{*}{$\begin{array}{c}\text { I.1. } \\
\text { Labor } \\
\text { employ } \\
\text { ment) }\end{array}$} & \multirow[b]{2}{*}{$\begin{array}{c}\text { I.2 } \\
\text { Capital }\end{array}$} & \multicolumn{2}{|c|}{ of which } & \multirow[b]{2}{*}{$\begin{array}{c}\text { Em- } \\
\text { ploy- } \\
\text { ment } \\
\text { struc- } \\
\text { ture }\end{array}$} & \multirow[b]{2}{*}{$\begin{array}{c}\text { II. Re- } \\
\text { main- } \\
\text { der }\end{array}$} \\
\hline & & & & & $\begin{array}{l}\text { Fixed } \\
\text { assets }\end{array}$ & $\begin{array}{l}\text { Load- } \\
\text { ing } \\
\text { rate }\end{array}$ & & \\
\hline 1 & 2 & 3 & 4 & 5 & 6 & 7 & 8 & 9 \\
\hline \multirow{2}{*}{$\begin{array}{l}\text { Bryansk } \\
\text { oblast }\end{array}$} & -2.91 & -1.13 & 0.14 & -1.27 & -0.30 & -0.97 & -0.99 & -0.79 \\
\hline & 7.90 & 1.47 & 0.44 & 1.06 & -0.18 & 1.23 & -0.56 & 6.99 \\
\hline \multirow{2}{*}{$\begin{array}{l}\text { Republic of } \\
\text { Kara- } \\
\text { chaevo- } \\
\text { Cherkessia }\end{array}$} & -1.69 & 0.15 & -0.28 & 0.43 & -0.15 & 0.58 & -0.69 & -1.15 \\
\hline & 10.39 & 1.09 & 1.43 & -0.55 & -0.10 & -0.44 & -0.71 & 10.01 \\
\hline \multirow{2}{*}{$\begin{array}{l}\text { Magadan } \\
\text { oblast }\end{array}$} & -9.01 & -4.24 & -3.72 & -0.52 & -0.05 & -0.47 & -0.63 & -4.15 \\
\hline & 3.10 & -2.84 & -0.73 & -2.13 & -0.09 & -2.04 & 0.37 & 5.57 \\
\hline \multirow{2}{*}{$\begin{array}{l}\text { Altay Re- } \\
\text { public }\end{array}$} & -3.40 & -0.25 & -0.13 & -0.12 & -0.12 & - & -1.62 & -1.52 \\
\hline & 7.29 & 0.45 & 0.25 & 0.15 & 0.15 & - & 0.02 & 6.82 \\
\hline
\end{tabular}




\begin{tabular}{lcccccccc}
\hline \multicolumn{1}{c}{1} & 2 & 3 & 4 & 5 & 6 & 7 & 8 & 9 \\
\hline Republic of & -7.24 & -0.59 & -0.19 & -0.41 & -0.41 & - & -3.76 & -2.88 \\
Ingoushetia & 3.33 & 2.26 & 3.55 & -0.36 & -0.36 & -41.65 & 0.79 & 0.28 \\
Republic of & -2.03 & -2.17 & -1.11 & -1.06 & 0.02 & -1.08 & -0.76 & 0.90 \\
Komi & 4.47 & 1.32 & 0.58 & 0.76 & 0.29 & 0.47 & -0.09 & 3.24 \\
Republic of & -3.20 & -15.45 & -0.06 & -15.39 & -0.12 & -15.28 & -0.77 & 13.03 \\
Khakassia & 2.53 & 17.86 & 0.76 & 15.92 & -0.12 & 16.04 & -0.58 & -14.75 \\
& -3.49 & -0.47 & -0.90 & 0.42 & -0.13 & 0.56 & -0.85 & -2.18 \\
Tula oblast & 5.11 & -0.03 & -0.36 & 0.36 & -0.01 & 0.36 & 0.13 & 5.02 \\
Chukotka & -14.52 & -5.99 & -5.65 & -0.34 & -0.34 & - & -0.36 & -8.17 \\
autonomous & 21.22 & -0.82 & -0.72 & 0.01 & 0.01 & -5.83 & 0.32 & 21.72 \\
okrug & & & & & & & &
\end{tabular}

In most regions whose dynamics of output o display the existence of the two periods undergo the following situation: the main reason for the drop in their output at the stage of decline is formed by basic factors - that is, labor and capital, while in the period of growth the remainder begins to prevail. An analogous result was found in the course of analysis of their annual growth rates.

\subsubsection{Decomposition of Growth with Account of Worked Time}

As noted above, assessment of labor input on the basis of the employment indicator suggests that the volume of output is equal for all the employees, regardless of the length of the workday. A more accurate assessment of labor input is built upon the total number of hours worked by all the employees. In this case changes in labor input (the aggregate worked time) are determined by two components: changes in the number of the employed and changes in the average length of the workday per employee (an average worked time):

$$
N^{i} \Rightarrow N_{h}^{i}=N^{i} \cdot H^{i},
$$


where $H^{i}$ - the time worked by a employee in $i$ region.

The index of the averaged worked time was built on the basis of the data on the average actual length of the working week ${ }^{37}$. In the assumption that the number of working weeks in the year is constant, the index of time worked by an individual employee in a year coincides with the index of actual length of the working week

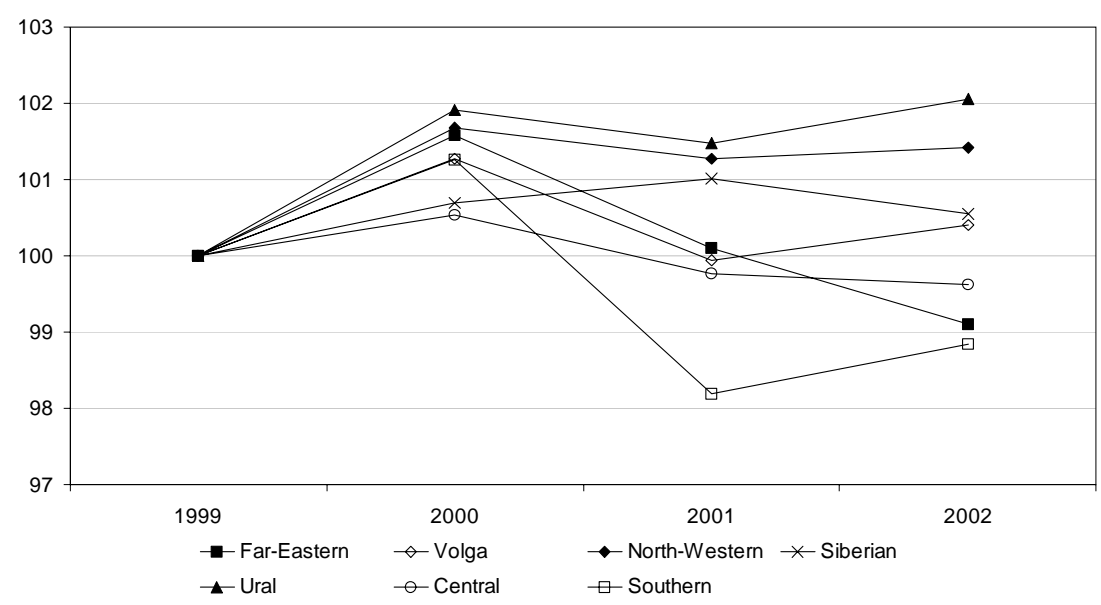

Fig. 3.14. The Dynamics of Indices of the Average Worked Time for the Federal Okrugs $(1999=100 \%)$

By contrast to the number of the employed, whose dynamics for most of regions and federal okrugs displays growth over the whole period between 2000 and 2002 (Fig. 3.14), the dynamics of the average worked time lacks such an explicitly manifested tendency. The rise of the working time of the employed in 2000 was followed by a fall in the indicator in 2001 (for all the federal okrugs, except the Siberian one).

${ }^{37}$ Source: The GKS' collection of surveys "Population Surveys on Employment Challenges”. 
Having transformed the expression for assessing the contribution of labor input in the rise in output in $i$ region, one can proceed to the sum of two components: namely, 1) change in the number of the employed $\left(\alpha_{L}^{i} g_{N^{i}}\right)$, and 2) change in the length of the working week $\left(\alpha_{L}^{i} g_{H^{i}}\right)$ :

$$
\alpha_{L}^{i} g_{N_{h}^{i}}=\alpha_{L}^{i} g_{N^{i} \cdot H^{i}}=\alpha_{L}^{i}\left(g_{N^{i}}+g_{H^{i}}\right) \text {. }
$$

While building assessments of the contribution of labor input in the growth of output, proceeding from the available statistical data, we have taken into account changes in the structure of the employed in terms of gender. The respective index is built in an assumption that the average hourly wages in each group are proportional to the marginal labor product per one worked hour.

$$
N_{h}^{i} \Rightarrow N_{h g}^{i}=N^{i} \cdot H^{i}\left(\frac{w_{m}^{i}}{\bar{w}^{i}} \frac{H_{m}^{i}}{H^{i}}+\frac{w_{f}^{i}}{\bar{w}^{i}} \frac{H_{f}^{i}}{H^{i}}\right),
$$

where

$w_{m}^{i}$ - men's average monthly wages in i region,

$w_{f}^{i}$ - women's average monthly wages in i region,

$\bar{w}^{i}$ - average monthly wages of the employed in i region,

$H_{m}^{i}$ - men's average actual length of the working week in i region,

$H_{f}^{i}$ - women's average actual length of the working week in i region.

Having transformed the expression to assess of the contribution of labor input with account of changes in gender structure of the employed to the rise in output in $i$ region and denoted 
$S t r^{i}=\frac{w_{m}^{i}}{\bar{w}^{i}} \frac{H_{m}^{i}}{H^{i}}+\frac{w_{f}^{i}}{\bar{w}^{i}} \frac{H_{f}^{i}}{H^{i}}$,

we receive:

$\alpha_{L}^{i} g_{N_{h g}^{i}}=\alpha_{L}^{i} g_{N^{i} \cdot H^{i} S t r^{i}}=\alpha_{L}^{i}\left(g_{N^{i}}+g_{H^{i}}+g_{S t r^{i}}\right)$.

Table 3.11

Results of Assessment of Output Growth Rates and Contribution of Labor Input by the Federal Okrugs

\begin{tabular}{|c|c|c|c|c|c|c|c|c|c|}
\hline \multirow[b]{2}{*}{$\begin{array}{c}\text { Federal } \\
\text { okrug }\end{array}$} & \multicolumn{5}{|c|}{ Growth rates } & \multicolumn{4}{|c|}{ As \% of the GRP growth rates } \\
\hline & $\begin{array}{l}\text { Number } \\
\text { of the } \\
\text { employed }\end{array}$ & $\begin{array}{c}\text { Aver- } \\
\text { age } \\
\text { worked } \\
\text { time }\end{array}$ & $\begin{array}{c}\text { Gender } \\
\text { structure }\end{array}$ & $\begin{array}{c}\text { Labor } \\
\text { input }\end{array}$ & GRP & $\begin{array}{c}\text { Number } \\
\text { of the } \\
\text { employed }\end{array}$ & $\begin{array}{c}\text { Aver- } \\
\text { age } \\
\text { worked } \\
\text { time } \\
\end{array}$ & $\begin{array}{c}\text { Gender } \\
\text { structure }\end{array}$ & $\begin{array}{c}\text { Labor } \\
\text { input }\end{array}$ \\
\hline \multicolumn{10}{|c|}{ 2000-2002 } \\
\hline $\begin{array}{l}\text { Far- } \\
\text { Eastern }\end{array}$ & 0.81 & -0.24 & 1.55 & 2.12 & 4.13 & 19.48 & -5.75 & 37.51 & 51.25 \\
\hline Volga & 0.38 & 0.06 & 0.34 & 0.78 & 5.71 & 6.65 & 1.02 & 5.92 & 13.59 \\
\hline $\begin{array}{l}\text { North- } \\
\text { Western }\end{array}$ & 0.42 & 0.25 & -0.36 & 0.31 & 7.56 & 5.52 & 3.33 & -4.75 & 4.10 \\
\hline Siberian & 0.27 & 0.10 & 0.01 & 0.38 & 6.03 & 4.45 & 1.60 & 0.24 & 6.28 \\
\hline Ural & 0.36 & 0.31 & 0.87 & 1.54 & 7.39 & 4.92 & 4.14 & 11.74 & 20.80 \\
\hline Central & 0.14 & -0.06 & 1.28 & 1.37 & 8.26 & 1.75 & -0.69 & 15.55 & 16.61 \\
\hline Southern & 0.84 & -0.22 & 0.02 & 0.63 & 8.11 & 10.33 & -2.77 & 0.22 & 7.78 \\
\hline
\end{tabular}

Computations evidence (Table 3.11) that the adjustment of the assessment of labor with account of changes in the worked time has led to a rise in the proportion of the output growth rates explained by changes in the labor input for most federal okrugs in the period of growth of 2000-02 (except for the North-Western and Southern ones). Computations for regions are given in the Annex 2, Table A2-12).

Results of the decomposition of the regions' growth output with account of worked time are presented in Table 3.12. 
Table 3.12

\section{Decomposition of the GRP Growth by the Federal Okrugs for the period 2000-2002}

\begin{tabular}{lccccccc}
\hline & $\begin{array}{c}\text { Far- } \\
\text { Eastern }\end{array}$ & Volga & $\begin{array}{c}\text { North- } \\
\text { Western }\end{array}$ & Siberian & Ural & Central & Southern \\
\hline GRP & 4,13 & 5,71 & 7,56 & 6,03 & 7,39 & 8,26 & 8,11 \\
I. Factor inputs & 1,80 & 1,52 & 1,41 & 1,33 & 3,39 & 3,12 & 1,04 \\
I.1 Labor & 2,12 & 0,78 & 0,31 & 0,38 & 1,54 & 1,37 & 0,63 \\
$\quad$ Employment & 0,81 & 0,38 & 0,42 & 0,27 & 0,36 & 0,14 & 0,84 \\
$\quad$ Worked time & $-0,24$ & 0,06 & 0,25 & 0,10 & 0,31 & $-0,06$ & $-0,22$ \\
$\quad$ Structure & 1,55 & 0,34 & $-0,36$ & 0,01 & 0,87 & 1,28 & 0,02 \\
I.2 Capital & $-0,32$ & 0,74 & 1,10 & 0,95 & 1,85 & 1,75 & 0,41 \\
$\quad$ Fixed assets & 0,06 & 0,32 & 0,21 & $-0,18$ & 0,63 & 0,36 & 0,47 \\
$\quad$ Loading rate & $-0,39$ & 0,43 & 0,89 & 1,13 & 1,22 & 1,38 & $-0,06$ \\
II. Remained & 2,34 & 4,19 & 6,16 & 4,70 & 4,00 & 5,14 & 7,07 \\
$\quad$ The Solow re- & 3,26 & 5,01 & 6,94 & 5,94 & 6,39 & 7,76 & 6,80 \\
mainder & & & & & &
\end{tabular}

In the period between 2000 and 2002, the remainder growth rates were positive in all the federal okrugs. However, despite the fact that the conducted adjustment of the assessment of labor input has led to a fall in the proportion of the output growth rates unexplained by the basic factors, it still exceeds the explained proportion. The proportion of the output growth rates generated by the growth of the remainder for all the federal okrugs changes within the range 40 to $80 \%$. Results of the decomposition of the regions' growth are given in the Annex 2 (Table A2-13).

As before, the percentage of the output growth rates unexplained by the basic factors likewise appears substantially diverse across regions. With account of changes of the worked time, the remainder determines a greater proportion of the output growth rates vs. labor and capital inputs. There are only 9 regions that made an exception in this respect: (city of Moscow, Tyumen, Yaroslavl, Sam- 
ara, Vologda, Smolensk, Sakhakin and Kamchtka oblast, and Republic of Khakassia).

\subsection{The Dual Approach to Decomposition of Growth}

Whilst conducting a direct decomposition of the regions' economic growth, we proceeded from the assumption that the value of output is determined solely by the included in production function factors, i.e.

$$
\sum_{i=1}^{m} p_{i} Y_{i}=\sum_{j=1}^{n} q_{j} X_{j}
$$

where

$p_{i}$ and $Y_{i}$ - price for and quantity of $i$ kind of product,

$q_{j}$ and $X_{j}$ - price for and quantity of $j$ kind of input.

In their paper, Griliches and Jorgenson (Griliches, Jorgenson (1967)) demonstrated that the assumption of the equality between the values of output and inputs allows another way to assess growth rates of total factor productivity which is computed as the total output to total inputs ratio:

$$
g_{\text {TFP }}=\sum_{j=1}^{n} v_{j} \frac{\dot{q}_{j}}{q_{j}}-\sum_{i=1}^{m} \mu_{i} \frac{\dot{p}_{i}}{p_{i}} .
$$

$\mu_{i}=\frac{p_{i} Y_{i}}{\sum p_{i} Y_{i}}$ and $v_{j}=\frac{q_{j} X_{j}}{\sum q_{j} X_{j}}$ are the shares of $i$ kind of product in the value of total output and the shares of $j$ kind of input in their value of total input, respectively.

Thus, the assessment of the growth of total factor productivity can be made basing on both indices of output and inputs values, and indices of the respective prices. These two methods ensure the same result, providing equality (3.13a) is met. In reality, as demonstrated by Hsieh (2002), this particular precondition appears fairly 
strict, especially as long as developing economies are concerned. In this paper, we attempt to decompose growth on the basis of the dual approach for the purpose of comparing its results with those of the direct approach. However, the problem with data still persists, which is why all the computations are built on the basis of aggregated indices, while if they are missing,- on the basis of estimates and proxy variables.

To measure the price rise of the aggregated outcome we employ the GRP deflator computed basing on the data of the index of physical volume of GRP and volume of GRP in constant prices ${ }^{38}$.

According to (3.14), the index of total factor productivity (unexplained by the basic factors remainders) on the basis of aggregated price indices is computed by the formula:

$$
\ln \left(\frac{A_{t}}{A_{t-1}}\right)=\left(\bar{\alpha}_{L} \ln \left(\frac{w_{t}}{w_{t-1}}\right)+\bar{\alpha}_{K} \ln \left(\frac{r_{t}}{r_{t-1}}\right)\right)-\ln \left(\frac{P_{t}^{Y}}{P_{t-1}^{Y}}\right),
$$

where, as before,

$\bar{\alpha}_{L}$ and $\bar{\alpha}_{K}$ - average for the two periods values of shares of the respective factors in output: (see. (3.4)),

$w$ and $r$-aggregated indices of labor and capital prices,

$P_{t}^{Y}$ - aggregated index of output price.

After transforming the above, we receive:

$$
\ln \left(\frac{A_{t}}{A_{t-1}}\right)=\bar{\alpha}_{L} \ln \left(\frac{\left(w / P^{Y}\right)_{t}}{\left(w / P^{Y}\right)_{t-1}}\right)+\bar{\alpha}_{K} \ln \left(\frac{\left(r / P^{Y}\right)_{t}}{\left(r / P^{Y}\right)_{t-1}}\right) \text {. }
$$

The first item in the right part of the expression represents adjusted by the weight coefficient logarithmic growth rates of real

\footnotetext{
${ }^{38}$ Source: "Regiony Rossii. Sotsialno-ekonomicheskie pokazateli - 2004 g." Rosstat.
} 
wages, while the other item represents adjusted by the weight coefficient logarithmic growth rates of the real rate of return on capital. Thus, the TFP growth rates are computed as a weighted sum of the real growth rate of labor and capital costs.

To assess the price index of labor, we employed the index of real average accrued wages (as \% to the prior year).

As in the case of assessing TFP by the direct method, the assessment of the capital index likewise forms more complex a task vis-à-vis an assessment of the labor index. First, this is explained by the absence of necessary statistics and, second, the ambiguity of the finding of technological efficiency of capital of different kinds and generations (vintages). In conjunction with that, the paper suggests two assessments of the price index of capital:

1. on the basis of investment goods prices, and

2. on the basis of prices for real estate on the secondary market.

The assumption that the capabilities of capital to produce output does not change in the process of its ageing and, consequently, the service price of capital do not change with its age suggests a price index for investment goods computed on the basis of index of physical volume of investment and volume of investment in constant prices ${ }^{39}$ as an acceptable estimate of the price index of capital.

Between 1998-2002 facilities and buildings roughly averaged $60 \%$ of fixed assets in the industrial sector and $71 \%$ in the agrarian sector (Table 3.13). Given that facilities and buildings account for the lion's share of fixed assets, the assessment of the service price of capital indeed can appear seriously correlated to prices for real estate.

39 Source: "Regiony Rossii. Sotsialno-ekonomicheskie pokazateli - 2004 g.”
Rosstat. 
Table 3.13

Structure of Fixed Assets by Kinds in the Industrial and Agrarian Sectors

\begin{tabular}{|c|c|c|c|c|c|c|}
\hline $\begin{array}{c}\text { All } \\
\text { fixed } \\
\text { assets }\end{array}$ & $\begin{array}{c}\text { Build- } \\
\text { ings }\end{array}$ & $\begin{array}{l}\text { Facilities } \\
\text { (includ- } \\
\text { ing } \\
\text { transmit- } \\
\text { ting ones) }\end{array}$ & $\begin{array}{l}\text { Machinery } \\
\text { and equip- } \\
\text { ment }\end{array}$ & $\begin{array}{c}\text { Means of } \\
\text { trans- } \\
\text { porta- } \\
\text { tion }\end{array}$ & $\begin{array}{l}\text { Work- } \\
\text { stock and } \\
\text { produc- } \\
\text { tive stock }\end{array}$ & $\begin{array}{l}\text { Other kinds } \\
\text { of fixed } \\
\text { assets }\end{array}$ \\
\hline
\end{tabular}

$\begin{array}{llllllll}1998 & 100 & 28.2 & 35.1 & 32.7 & 2.7 & - & 1,3 \\ 1999 & 100 & 27.7 & 35.5 & 32.9 & 2.7 & - & 1,2 \\ 2000 & 100 & 27.1 & 33.2 & 35.6 & 2.9 & - & 1,2 \\ 2001 & 100 & 24.7 & 32.9 & 37.7 & 3.4 & - & 1,3 \\ 2002 & 100 & 20.7 & 37.9 & 37.1 & 3.1 & - & 1,2\end{array}$

Agrarian sector

$\begin{array}{llccccc}1998 & 100 & 74 & 17.2 & 4 & 2.5 & 2.3 \\ 1999 & 100 & 73.4 & 17.3 & 4 & 2.9 & 2.4 \\ 2000 & 100 & 72.3 & 17.7 & 4.2 & 3.5 & 2.3 \\ 2001 & 100 & 70.6 & 18.4 & 4.3 & 4.3 & 2.4 \\ 2002 & 100 & 68.1 & 19.8 & 4.5 & 5.2 & 2.4\end{array}$

The price index in secondary market of dwellings was employed to build the price index for buildings and facilities ${ }^{40}$.

Thus, while finding the remainder growth rates by means of the dual method, we considered two options for building the assessment:

- on the basis of the GRP deflator as the output price index; and the price index for facilities and buildings as the price index of capital;

- on the basis of the GRP deflator as the output price index; and the price index of investment goods as the price index of capital .

40 Source: "Regiony Rossii. Sotsialno-ekonomicheskie pokazateli - 2004 g."
Rosstat. 


\subsubsection{Comparing Assessments Made by Different Methods}

The 1997-2002 unexplained remainder assessment results of the by the basic factors of regions' economic growth (TFP) are given in Table 3.14, while those of 1997-98 and 1999-2000 - in the Annex 2(Table 2A-15 and 2A-16).

Table 3.14

Results of Assessments of Remainder Growth Rates for the Period between 1997 and 2002

\begin{tabular}{|c|c|c|c|c|c|c|}
\hline & $\begin{array}{l}\text { TFP (So- } \\
\text { low) }\end{array}$ & $\begin{array}{l}\text { TFP (Direct } \\
\text { method) }\end{array}$ & $\begin{array}{l}\text { TFP (dual } \\
\text { method 1) }\end{array}$ & $\begin{array}{l}\text { TFP (dual } \\
\text { method 2) }\end{array}$ & $\min$ & $\max$ \\
\hline 1 & 2 & 3 & 4 & 5 & 6 & 7 \\
\hline Russian Federation & 3.45 & & 2.85 & 1.14 & 1.14 & 3.45 \\
\hline Altay Krai & 0.80 & 2.71 & 0.87 & -2.53 & -2.53 & 2.71 \\
\hline Amour Oblast & 0.51 & 1.74 & 4.07 & 2.18 & 0.51 & 4.07 \\
\hline Arkhangel' Oblast & 4.38 & 4.44 & 3.44 & 1.14 & 1.14 & 4.44 \\
\hline Astrakhan Oblast & 6.79 & 7.42 & & 3.20 & 3.20 & 7.42 \\
\hline Belgorod Oblast & 4.63 & 4.08 & 1.07 & 0.43 & 0.43 & 4.63 \\
\hline Bryansk Oblast & 2.23 & 2.91 & 3.06 & -0.77 & -0.77 & 3.06 \\
\hline Vladimir Oblast & 2.90 & 2.57 & 3.31 & 2.71 & 2.57 & 3.31 \\
\hline Volgograd Oblast & 2.62 & 5.62 & -0.87 & -2.70 & -2.70 & 5.62 \\
\hline Vologda Oblast & 2.91 & 2.54 & 2.06 & -0.18 & -0.18 & 2.91 \\
\hline Voronezh Oblast & 1.96 & 2.85 & -0.61 & -1.29 & -1.29 & 2.85 \\
\hline the city of Moscow & 4.92 & 2.81 & -4.44 & -7.69 & -7.69 & 4.92 \\
\hline $\begin{array}{l}\text { the city of Saint- } \\
\text { Petersburg }\end{array}$ & 4.36 & 2.82 & 1.94 & 2.64 & 1.94 & 4.36 \\
\hline $\begin{array}{l}\text { Jewish Autonomous } \\
\text { Oblast }\end{array}$ & -0.74 & 0.19 & & 0.16 & -0.74 & 0.19 \\
\hline Ivanovo Oblast & 0.97 & 0.89 & 3.05 & 0.54 & 0.54 & 3.05 \\
\hline Irkutsk Oblast & -1.93 & -1.74 & 6.89 & 0.18 & -1.93 & 6.89 \\
\hline $\begin{array}{l}\text { Kabardino-Balkar Re- } \\
\text { public }\end{array}$ & 5.63 & 7.56 & 2.25 & 2.20 & 2.20 & 7.56 \\
\hline Kaliningrad Oblast & 2.53 & 2.64 & -4.60 & -3.49 & -4.60 & 2.64 \\
\hline Kaluga Oblast & 1.62 & 1.44 & 2.81 & 2.43 & 1.44 & 2.81 \\
\hline Kamchatka Oblast & -2.36 & -0.94 & 1.41 & -5.36 & -5.36 & 1.41 \\
\hline $\begin{array}{l}\text { Karachay-Cherkessya } \\
\text { Republic }\end{array}$ & 3.75 & 4.27 & -0.97 & 2.49 & -0.97 & 4.27 \\
\hline
\end{tabular}




\begin{tabular}{|c|c|c|c|c|c|c|}
\hline 1 & 2 & 3 & 4 & 5 & 6 & 7 \\
\hline Kemerovo Oblast & 2.29 & 2.49 & 2.53 & -0.70 & -0.70 & 2.53 \\
\hline Kirov Oblast & -0.13 & 0.11 & 3.11 & 0.53 & -0.13 & 3.11 \\
\hline Kostroma Oblast & 2.32 & 2.77 & & 0.18 & 0.18 & 2.77 \\
\hline KrasnodarKrai & 2.08 & 1.68 & 0.47 & -1.92 & -1.92 & 2.08 \\
\hline Krasnoyarsk Krai & 2.06 & 2.68 & 2.74 & -2.65 & -2.65 & 2.74 \\
\hline Kurgan Oblast & 1.69 & 2.95 & 0.84 & -1.83 & -1.83 & 2.95 \\
\hline Kursk Oblast & 2.94 & 3.51 & & 1.45 & 1.45 & 3.51 \\
\hline Leningrad Oblast & 5.71 & 5.50 & & 2.40 & 2.40 & 5.71 \\
\hline Lipetsk Oblast & 1.99 & 3.35 & -1.72 & -2.47 & -2.47 & 3.35 \\
\hline Magadan Oblast & -1.03 & -0.06 & & -2.36 & -2.36 & -0.06 \\
\hline Moscow Oblast & 3.93 & 4.05 & 4.94 & -0.05 & -0.05 & 4.94 \\
\hline Murmansk Oblast & 2.20 & 2.96 & & -2.24 & -2.24 & 2.96 \\
\hline $\begin{array}{l}\text { Nizhny Novgorod } \\
\text { Oblast }\end{array}$ & 4.05 & 4.04 & -0.39 & -3.03 & -3.03 & 4.05 \\
\hline Novgorod Oblast & 4.18 & 4.43 & 0.03 & 1.15 & 0.03 & 4.43 \\
\hline Novosibirsk Oblast & 3.84 & 4.94 & 4.88 & -1.30 & -1.30 & 4.94 \\
\hline Omsk Oblast & 1.38 & 2.36 & 4.57 & 1.37 & 1.37 & 4.57 \\
\hline Orenburg Oblast & 1.52 & 1.56 & 1.12 & -1.60 & -1.60 & 1.56 \\
\hline Orel Oblast & 5.39 & 6.08 & 4.04 & 1.52 & 1.52 & 6.08 \\
\hline Penza Oblast & 1.93 & 3.67 & 3.27 & 1.28 & 1.28 & 3.67 \\
\hline Perm Oblast & 3.27 & 3.32 & 2.10 & 0.00 & 0.00 & 3.32 \\
\hline Primorsky Krai & 0.18 & 0.73 & -0.92 & 0.35 & -0.92 & 0.73 \\
\hline Pskov Oblast & 0.81 & 1.95 & -0.90 & -1.21 & -1.21 & 1.95 \\
\hline Republic Adygeya & 0.41 & 1.68 & 1.71 & 1.83 & 0.41 & 1.83 \\
\hline Altay Republic & 1.78 & 2.60 & 1.26 & -0.82 & -0.82 & 2.60 \\
\hline Republic Bashkortostan & 1.52 & 2.74 & 2.31 & -0.90 & -0.90 & 2.74 \\
\hline Republic Buryatia & 4.63 & 6.10 & -0.48 & 0.42 & -0.48 & 6.10 \\
\hline Republic of Dagestan & 3.55 & 4.41 & -0.67 & 0.05 & -0.67 & 4.41 \\
\hline Republic of Ingoushetia & -3.79 & -1.86 & & -11.39 & -11.39 & -1.86 \\
\hline Republic of Kalmykia & 6.06 & 11.40 & & -7.44 & -7.44 & 11.40 \\
\hline Republic Karelia & 3.31 & 2.76 & 1.32 & 0.56 & 0.56 & 3.31 \\
\hline Republic Komi & 1.28 & 2.03 & 3.99 & 0.76 & 0.76 & 3.99 \\
\hline Republic Mary-El & -0.28 & 2.73 & 3.66 & 0.02 & -0.28 & 3.66 \\
\hline Republic Mordovia & 4.27 & 4.43 & & -0.06 & -0.06 & 4.43 \\
\hline
\end{tabular}




\begin{tabular}{|c|c|c|c|c|c|c|}
\hline 1 & 2 & 3 & 4 & 5 & 6 & 7 \\
\hline $\begin{array}{l}\text { Republic of Sakha (Ya- } \\
\text { kutia) }\end{array}$ & 2.03 & 2.87 & 0.45 & -2.59 & -2.59 & 2.87 \\
\hline $\begin{array}{l}\text { Republic North Ossetia } \\
\text { (Alania) }\end{array}$ & 5.42 & 6.21 & - & -2.34 & -2.34 & 6.21 \\
\hline Republic Tatarstan & 2.74 & 2.80 & -4.31 & -1.49 & -4.31 & 2.80 \\
\hline Republic of Tyva & 5.49 & 5.79 & & 0.09 & 0.09 & 5.79 \\
\hline Republic Khakassia & -0.60 & -2.23 & 1.72 & -4.01 & -4.01 & 1.72 \\
\hline Rostov Oblast & 5.88 & 6.78 & 2.45 & 0.03 & 0.03 & 6.78 \\
\hline Ryazan Oblast & 2.58 & 3.03 & 3.27 & 0.63 & 0.63 & 3.27 \\
\hline Samara Oblast & 2.17 & 2.18 & -2.53 & -0.70 & -2.53 & 2.18 \\
\hline Saratov Oblast & 3.84 & 4.80 & 0.81 & -0.22 & -0.22 & 4.80 \\
\hline Sakhalin Oblast & 2.00 & 4.38 & 1.38 & -3.01 & -3.01 & 4.38 \\
\hline Sverdlovsk Oblast & 2.00 & 1.66 & 2.45 & -0.16 & -0.16 & 2.45 \\
\hline Smolensk Oblast & 3.75 & 4.68 & - & 5.25 & 3.75 & 5.25 \\
\hline Stavropol Krai & 2.62 & 3.09 & 5.09 & 3.96 & 2.62 & 5.09 \\
\hline Tambov Oblast & 5.91 & 7.25 & 2.72 & -1.30 & -1.30 & 7.25 \\
\hline Tver Oblast & 1.55 & 2.09 & 0.36 & 1.86 & 0.36 & 2.09 \\
\hline Tomsk Oblast & 3.17 & 2.99 & 3.80 & -0.57 & -0.57 & 3.80 \\
\hline Tula Oblast & 1.47 & 1.36 & -0.54 & -0.26 & -0.54 & 1.47 \\
\hline Tyumen Oblast & 3.16 & 1.27 & 1.32 & -2.49 & -2.49 & 3.16 \\
\hline Udmurtia Republic & 1.69 & 1.42 & 2.00 & -2.04 & -2.04 & 2.00 \\
\hline Ulyanovsk Oblast & 2.16 & 3.51 & 4.79 & -0.19 & -0.19 & 4.79 \\
\hline Khabarovsk Krai & 4.15 & 4.65 & 5.03 & -0.98 & -0.98 & 5.03 \\
\hline Chelyabinsk Oblast & 0.55 & 2.32 & -1.88 & -1.37 & -1.88 & 2.32 \\
\hline Chita Oblast & 0.95 & 1.95 & 5.13 & 0.99 & 0.95 & 5.13 \\
\hline Chuvash Republic & -0.45 & -0.74 & 4.96 & 1.21 & -0.74 & 4.96 \\
\hline $\begin{array}{l}\text { Chukotka autonomous } \\
\text { okrug }\end{array}$ & 5.54 & 5.65 & - & -9.51 & -9.51 & 5.65 \\
\hline Yaroslavl Oblast & 4.28 & 3.40 & 7.16 & 3.09 & 3.09 & 7.16 \\
\hline
\end{tabular}

The assessments of the remainder growth rates made by different methods display a great deal of diversity. The minimum and maximum values of the assessments have opposite signs for rather a great number of regions. For 27 regions (Amur, Arkhangel'sk, Astrakhan, Belgorod, Vladimir oblasts, St. Petersburg, among oth- 
ers) both margins of the assessment of the remainder growth rates are positive, while those for Magadan oblast and Republic of Ingoushetia are negative. In 55 regions (from 79 concerned) the lower assessment margin was found by means of the dual assessment method basing on GRP deflator and price index for investment goods. The upper margin of the assessment of the remainder growth rate in more than half of the regions was found by means of direct method with account of changes in the capacity loading rate.

Fig. 3.15 presents diagrams of dispersion of the respective TFP assessments with GRP growth rates.

Similarly, the analysis of the correlation between GRP growth rates and those of remainder between 1997 and 2002 leads to different results. The assessing of the remainder by the direct method exposes the existence of an explicit linear correlation between the GRP growth rates and those of the remainder, which testifies to the fact that a major part of growth rates are not determined by the basic factors. The assessment of the remainder by the dual method fails to identify such a correlation, while the analysis of growth rates for the periods 1997-98 and 1999-2002 leads to analogous results (see Annex 2, Figs. 2A-8 and 2A-9). 


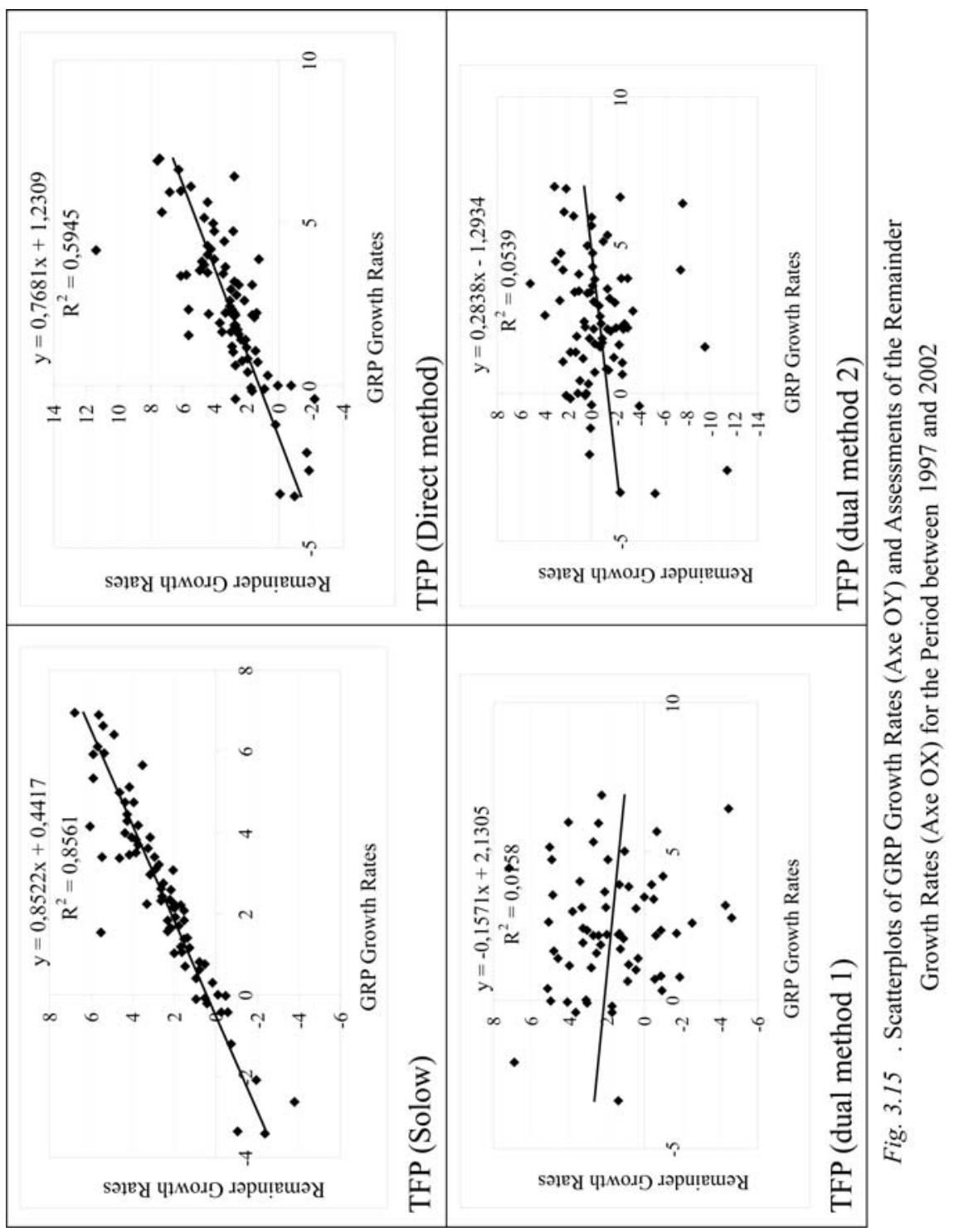




\subsection{Labor Productivity in the Industrial Sector}

The above assessments demonstrated that decomposition of growth of value indicators, such as GRP, in regional terms allows only rough and often confusing (under employment of different methods) assessment of growth in productivity. Let us note that while explaining value indicators on the nationwide level (GDP, sectoral GVA), in the course of decomposition of growth one also noted rather a great unexplained remainder (some 70\%). While on the one hand this result evidences great measure errors, it questions prerequisites that underlie the decomposition, on the other.

The strongest prerequisite is likely to be the assumption of factor prices being equal to their marginal products and meeting the condition of long-term equilibrium, which appears doubtful in the case of Russia. Indeed, as demonstrated by Hsieh (2002), this prerequisite is likely to appear stronger for developing economies than for developed ones, which manifests itself in the difference between assessments of the direct and dual methods of decomposition of growth.

The IET papers (Entov, Lugovoy at al. (2003)) also demonstrated that once completed, a decomposition of growth on the basis of physical indicators provides a smaller remainder, even providing aggregated inputs are employed. By contrast to physical values, price indicators are more volatile and dependent on rapidly changing prices revenues can experience dramatic changes under a constant physical volume of output.

Unfortunately, employment of the physical volume indicators is impossible on the level of a nation or region's economy as a whole. The physical volumes indices can be computed relatively accurately just for a few individual industries that produce a homogenous product whose quality grows over time. The index of physical volume of industrial output (IPI) can form some approximation of such an index. 
The IPI dynamics by regions and Russia as a whole are given on Fig. 3.16 (see also Annex 2, Map 4). One can note that the turning point of the trend to decline in industrial output in Russia occurred in 1998 on average, while the regional dynamics vary by regions. While some regions saw the turn of the trend in 1995, some others as late as in 2000. However, the decline at the beginning of the period in question and rise at its end is noted in all the regions. Let us also note that in 2003 it was only a few regions (Arkhangel'sk, Belgorod, Leningrad, Tomsk oblasts and Nenetsky AO) that reached and surpassed the 1990 level of output, while the averaged volume of output across Russia accounted for less than $70 \%$ of its 1990 level.

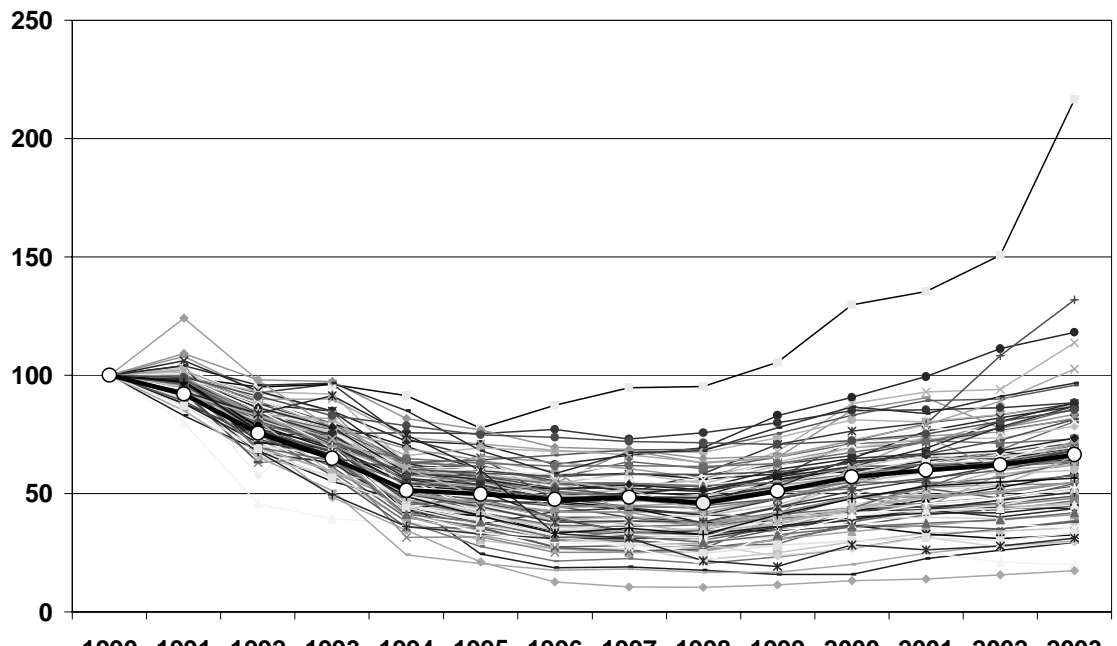

Fig. 3.16. The Dynamics of the Industrial Output Index by Regions of the Russian Federation in 1990-2003 $(1990=100)$

The conduct of decomposition of growth in industrial output, again, necessitates the respective assessments of labor and capital inputs. Given a complete absence of acceptable statistics by fixed 
assets in regions, it is possible to assess just labor productivity (the output to the employees in an industry ratio). The respective assessments are given in Fig. 3.17 (see also Annex 2, Map 5).

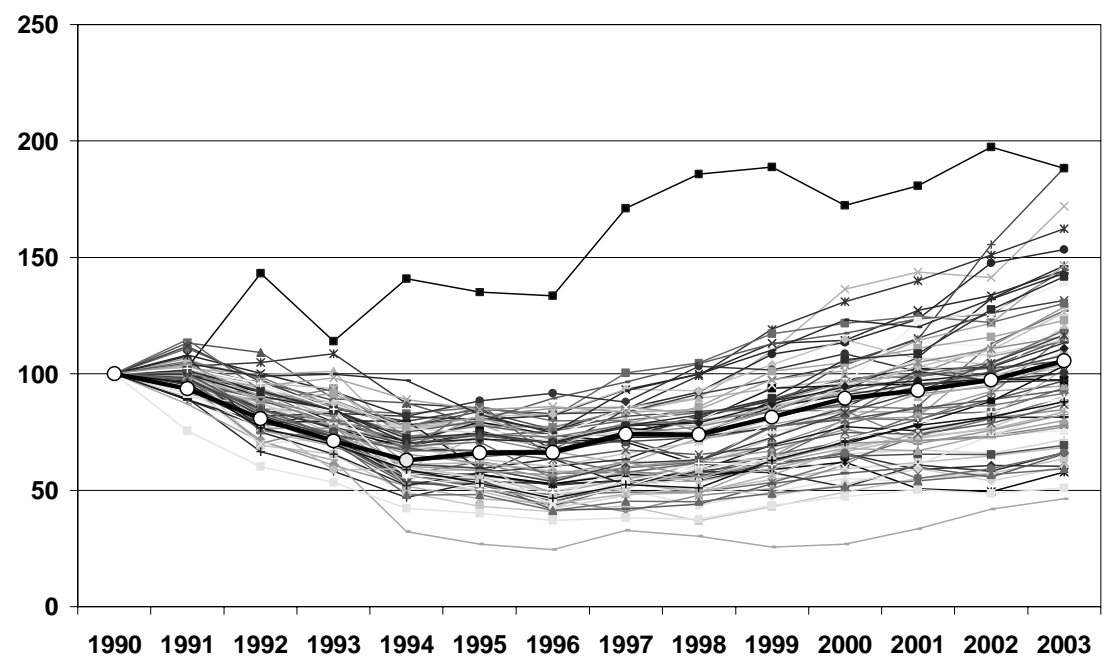

Fig. 3.17. The Dynamics of the labor Productivity Index in the Industrial Sector by Regions of Russian Federation in 1990-2003 (1990=100).

As shown by computations (see Annex 2, Table 2A-17), the turn of the trend in labor productivity in the industrial sector from decline to growth occurred in 1994 on average, or in 4 years earlier than the change in the output trend, while in some regions labor productivity had a positive trend through the whole period of question.

Importantly, labor productivity in the industrial sector exceeded the 1990 level in a major part of regions.

These results match sector-level computations and those made on the level of the economy as a whole (Entov, Lugovoy at al. (2003)). The trend to rise in productivity began taking shape much earlier than growth in output. 
As landmarks for a further analysis that should form "a second step" of the research into sources of growth we can suggest a statistical examination of the impact a broad array of factors have on the growth in output, basic inputs and productivity.

Preliminary comparisons between the received assessment of labor productivity in the industrial sector and some factors show that the labor productivity growth rate in regions is positively correlated with the volume of foreign investment and negatively - with financial aid granted to the regions.

Whereas capital forms one of the basic factors that affect labor productivity, a positive correlation between productivity and investment once again proves that labor productivity growth rate was greater in regions with relatively greater foreign investment.

A negative correlation between the federal financial aid and labor productivity can be attributed both to the effect the financial aid has on productivity and vice versa - the aid was allocated primarily to depressive regions with a smaller productivity growth rate, which requires an additional examination. 


\section{Annex 2}

Table 2A-1

Decomposition of Growth in GVA in Industry (MPL=0,7, MPK=0,3)

\begin{tabular}{lcccccc}
\hline & \multicolumn{2}{c}{ 1992-98 } & & \multicolumn{2}{c}{ 1999-03 } & \multicolumn{2}{c}{ 1992-03 } \\
\hline GVA & -11.75 & - & 6.43 & - & -4.59 & - \\
I. Factor inputs & -8.28 & - & 3.25 & - & -3.63 & - \\
I.1 Labor & -5.69 & - & 1.31 & - & -2.82 & - \\
$\quad$ Employment & - & -4.44 & - & -0.02 & & -2.63 \\
Worked time & - & -1.25 & - & 1.33 & & -0.19 \\
I.2 Capital & -2.58 & & 1.94 & - & -0.81 & - \\
Inventories & - & -0.69 & - & -0.06 & - & -0.39 \\
Fixed assets & - & 0.06 & - & 0.08 & - & 0.07 \\
Loading rate & - & -1.95 & - & 1.92 & - & -0.49 \\
II. TFP & -3.47 & & 3.18 & - & -0.96 & - \\
$\quad$ Price factor & - & -2.25 & - & -1.26 & - & -1.89 \\
$\quad$ Remainder & - & -1.22 & - & 4.44 & - & 0.94 \\
\hline
\end{tabular}

Table 2A-2

Decomposition of Growth in GDP

\begin{tabular}{lcccccc}
\hline & \multicolumn{2}{c}{ 1993-98 } & & \multicolumn{2}{c}{ 1999-03 } & \multicolumn{2}{c}{ 1993-03 } \\
\hline GDP & -7.30 & - & 6.56 & - & -1.3 & - \\
I. Factor inputs & -3.46 & - & 1.79 & - & -0.96 & - \\
I.1 Labor & -1.46 & - & 0.06 & - & -0.56 & - \\
$\quad$ Employment & - & -0.84 & - & 0 & - & -0.33 \\
Worked time & - & -0.62 & - & 0.06 & - & -0.23 \\
I.2 Capital & -2 & - & 1.73 & - & -0.4 & - \\
$\quad$ Fixed assets & - & -0.05 & - & 0.41 & - & 0.12 \\
$\quad$ Loading rate & - & -1.95 & - & 1.32 & - & -0.52 \\
II. TFP & -3.835 & - & 4.77 & - & -0.34 & - \\
\hline
\end{tabular}


Table 2A-3

\section{Results of Computations of GRP Growth Indices by Regions on the Basis of Different Deflators}

\begin{tabular}{|c|c|c|c|c|c|}
\hline Regions & $\begin{array}{l}\text { Variant1: Index } \\
\text { of physical } \\
\text { volume of GRP }\end{array}$ & $\begin{array}{l}\text { Variant 2: Com- } \\
\text { putation on the } \\
\text { basis of GDP } \\
\text { deflator }\end{array}$ & $\begin{array}{l}\text { Variation (as \% } \\
\text { of variant } 1 \text { ) }\end{array}$ & $\begin{array}{l}\text { Variant 3: com- } \\
\text { putation on the } \\
\text { basis of regions' } \\
\text { CPI }\end{array}$ & $\begin{array}{c}\text { Variation (as \% } \\
\text { of variant 1) }\end{array}$ \\
\hline 1 & 2 & 3 & 4 & 5 & 6 \\
\hline Russian Federation & 123.95 & 111.61 & -9.95 & 106.33 & -14.21 \\
\hline Altay Krai & 106.13 & 82.48 & -22.29 & 84.51 & -20.38 \\
\hline Amour Oblast & 102.19 & 85.18 & -16.64 & 98.38 & -3.72 \\
\hline Arkhangel' Oblast & 128.99 & 105.92 & -17.89 & 112.98 & -12.42 \\
\hline Astrakhan Oblast & 152.63 & 125.86 & -17.54 & 127.08 & -16.74 \\
\hline Belgorod Oblast & 135.95 & 100.59 & -26.01 & 97.81 & -28.06 \\
\hline Bryansk Oblast & 116.49 & 83.78 & -28.08 & 79.85 & -31.45 \\
\hline Vladimir Oblast & 121.46 & 95.11 & -21.70 & 98.85 & -18.62 \\
\hline Volgograd Oblast & 117.19 & 88.85 & -24.19 & 85.93 & -26.67 \\
\hline Vologda Oblast & 121.52 & 98.85 & -18.65 & 112.44 & -7.47 \\
\hline Voronezh Oblast & 114.88 & 100.13 & -12.84 & 99.68 & -13.23 \\
\hline the city of Moscow & 149.39 & 193.77 & 29.71 & 141.81 & -5.08 \\
\hline $\begin{array}{l}\text { the city of Saint- } \\
\text { Petersburg }\end{array}$ & 134.77 & 126.96 & -5.80 & 116.79 & -13.35 \\
\hline $\begin{array}{l}\text { Jewish Autonomous } \\
\text { Oblast }\end{array}$ & 95.98 & 102.79 & 7.10 & 108.77 & 13.33 \\
\hline Ivanovo Oblast & 101.51 & 78.65 & -22.52 & 79.28 & -21.90 \\
\hline Irkutsk Oblast & 89.40 & 74.41 & -16.77 & 81.12 & -9.26 \\
\hline $\begin{array}{l}\text { Kabardino-Balkar } \\
\text { Republic }\end{array}$ & 153.18 & 121.43 & -20.73 & 120.81 & -21.13 \\
\hline Kaliningrad Oblast & 120.40 & 125.55 & 4.28 & 122.24 & 1.53 \\
\hline Kaluga Oblast & 107.60 & 95.42 & -11.33 & 98.25 & -8.69 \\
\hline Kamchatka Oblast & 82.06 & 74.07 & -9.74 & 70.78 & -13.75 \\
\hline $\begin{array}{l}\text { Karachay-Cherkessya } \\
\text { Republic }\end{array}$ & 129.91 & 96.73 & -25.54 & 91.23 & -29.78 \\
\hline Kemerovo Oblast & 111.02 & 70.31 & -36.67 & 81.14 & -26.91 \\
\hline Kirov Oblast & 100.61 & 81.77 & -18.72 & 84.21 & -16.29 \\
\hline Kostroma Oblast & 112.15 & 90.98 & -18.88 & 91.99 & -17.97 \\
\hline KrasnodarKrai & 122.57 & 117.50 & -4.14 & 115.56 & -5.72 \\
\hline Krasnoyarsk Krai & 114.53 & 94.15 & -17.79 & 103.17 & -9.92 \\
\hline Kurgan Oblast & 108.46 & 88.00 & -18.87 & 78.93 & -27.23 \\
\hline
\end{tabular}




\begin{tabular}{|c|c|c|c|c|c|}
\hline 1 & 2 & 3 & 4 & 5 & 6 \\
\hline Kursk Oblast & 123.34 & 83.38 & -32.40 & 74.25 & -39.80 \\
\hline Leningrad Oblast & 147.24 & 130.75 & -11.20 & 129.74 & -11.89 \\
\hline Lipetsk Oblast & 115.49 & 108.14 & -6.36 & 108.93 & -5.67 \\
\hline Magadan Oblast & 83.76 & 87.80 & 4.82 & 96.96 & 15.75 \\
\hline Moscow Oblast & 133.30 & 121.98 & -8.49 & 115.87 & -13.08 \\
\hline Murmansk Oblast & 110.76 & 94.74 & -14.46 & 81.12 & -26.75 \\
\hline $\begin{array}{l}\text { Nizhny Novgorod } \\
\text { Oblast }\end{array}$ & 127.06 & 105.93 & -16.63 & 88.77 & -30.14 \\
\hline Novgorod Oblast & 123.93 & 103.51 & -16.47 & 108.49 & -12.46 \\
\hline Novosibirsk Oblast & 125.35 & 93.41 & -25.48 & 104.89 & -16.32 \\
\hline Omsk Oblast & 111.19 & 72.97 & -34.38 & 78.67 & -29.25 \\
\hline Orenburg Oblast & 115.67 & 91.32 & -21.05 & 93.03 & -19.57 \\
\hline Orel Oblast & 143.95 & 118.94 & -17.38 & 130.22 & -9.54 \\
\hline Penza Oblast & 114.13 & 90.17 & -20.99 & 84.24 & -26.18 \\
\hline Perm Oblast & 126.11 & 102.31 & -18.87 & 89.45 & -29.07 \\
\hline Primorsky Krai & 102.41 & 92.55 & -9.63 & 101.06 & -1.33 \\
\hline Pskov Oblast & 107.72 & 97.62 & -9.38 & 100.63 & -6.59 \\
\hline Republic Adygeya & 99.68 & 79.59 & -20.15 & 74.22 & -25.55 \\
\hline Altay Republic & 113.68 & 115.49 & 1.60 & 111.15 & -2.22 \\
\hline $\begin{array}{l}\text { Republic Bashkor- } \\
\text { tostan }\end{array}$ & 112.65 & 88.51 & -21.43 & 87.26 & -22.54 \\
\hline Republic Buryatia & 123.23 & 94.37 & -23.42 & 100.32 & -18.59 \\
\hline Republic of Dagestan & 143.52 & 161.45 & 12.49 & 167.29 & 16.56 \\
\hline $\begin{array}{l}\text { Republic of Ingou- } \\
\text { shetia }\end{array}$ & 96.56 & 104.00 & 7.70 & 81.08 & -16.03 \\
\hline Republic of Kalmykia & 146.27 & 234.97 & 60.64 & 222.33 & 52.00 \\
\hline Republic Karelia & 116.03 & 106.31 & -8.38 & 113.33 & -2.33 \\
\hline Republic Komi & 108.10 & 103.73 & -4.04 & 118.64 & 9.76 \\
\hline Republic Mary-El & 97.78 & 92.82 & -5.08 & 83.79 & -14.31 \\
\hline Republic Mordovia & 130.13 & 73.26 & -43.70 & 60.64 & -53.40 \\
\hline $\begin{array}{l}\text { Republic of Sakha } \\
\text { (Yakutia) }\end{array}$ & 107.07 & 96.73 & -9.66 & 125.14 & 16.88 \\
\hline $\begin{array}{l}\text { Republic North } \\
\text { Ossetia (Alania) }\end{array}$ & 150.73 & 120.89 & -19.80 & 123.95 & -17.76 \\
\hline Republic Tatarstan & 122.53 & 104.01 & -15.11 & 93.29 & -23.86 \\
\hline Republic of Tyva & 123.73 & 104.13 & -15.84 & 127.29 & 2.88 \\
\hline Republic Khakassia & 98.30 & 89.23 & -9.23 & 80.62 & -17.99 \\
\hline Rostov Oblast & 144.30 & 107.47 & -25.52 & 90.82 & -37.06 \\
\hline
\end{tabular}




\begin{tabular}{lccccc}
\hline \multicolumn{1}{c}{1} & 2 & 3 & 4 & 5 & 6 \\
\hline Ryazan Oblast & 116.46 & 100.98 & -13.29 & 100.02 & -14.11 \\
Samara Oblast & 117.76 & 91.65 & -22.18 & 89.38 & -24.10 \\
Saratov Oblast & 128.49 & 96.71 & -24.73 & 90.49 & -29.58 \\
Sakhalin Oblast & 118.86 & 134.49 & 13.16 & 135.37 & 13.90 \\
Sverdlovsk Oblast & 115.33 & 83.76 & -27.37 & 74.40 & -35.49 \\
Smolensk Oblast & 127.98 & 106.98 & -16.42 & 97.34 & -23.94 \\
Stavropol Krai & 118.06 & 88.72 & -24.85 & 82.56 & -30.07 \\
Tambov Oblast & 138.48 & 108.69 & -21.51 & 107.49 & -22.38 \\
Tver Oblast & 109.46 & 93.67 & -14.43 & 90.47 & -17.34 \\
Tomsk Oblast & 121.36 & 103.71 & -14.54 & 98.88 & -18.52 \\
Tula Oblast & 105.15 & 101.35 & -3.61 & 103.32 & -1.73 \\
Tyumen Oblast & 126.99 & 119.82 & -5.64 & 121.53 & -4.30 \\
Udmurtia Republic & 115.34 & 105.21 & -8.78 & 100.60 & -12.78 \\
Ulyanovsk Oblast & 111.42 & 75.49 & -32.25 & 61.30 & -44.98 \\
Khabarovsk Krai & 137.38 & 97.29 & -29.18 & 96.06 & -30.08 \\
Chelyabinsk Oblast & 107.67 & 86.41 & -19.75 & 77.06 & -28.43 \\
Chita Oblast & 105.01 & 89.14 & -15.12 & 92.86 & -11.57 \\
Chuvash Republic & 100.91 & 88.45 & -12.35 & 82.80 & -17.95 \\
Chukotka autono- & 124.75 & 116.67 & -6.47 & 92.36 & -25.96 \\
mous okrug & 132.56 & 109.96 & -17.05 & 111.01 & -16.25 \\
Yaroslavl Oblast & & & & &
\end{tabular}

Table 2A-4

Results of Computations of GRP Growth Indices by Regions
(on the Basis of Index of physical volume of GRP - variant 1)

\begin{tabular}{lccccccc}
\hline \multicolumn{1}{c}{ Region } & $\mathbf{1 9 9 6}$ & $\mathbf{1 9 9 7}$ & $\mathbf{1 9 9 8}$ & $\mathbf{1 9 9 9}$ & $\mathbf{2 0 0 0}$ & $\mathbf{2 0 0 1}$ & $\mathbf{2 0 0 2}$ \\
\hline \multicolumn{1}{c}{1} & 2 & 3 & 4 & 5 & 6 & 7 & 8 \\
\hline Altay Krai & 100 & 85.90 & 82.98 & 85.47 & 95.47 & 102.25 & 106.13 \\
Amour Oblast & 100 & 91.10 & 81.44 & 84.62 & 89.95 & 105.78 & 102.19 \\
Arkhangel' Oblast & 100 & 96.20 & 92.54 & 103.09 & 120.00 & 126.96 & 128.99 \\
Astrakhan Oblast & 100 & 104.00 & 105.25 & 117.56 & 134.61 & 145.92 & 152.63 \\
Belgorod Oblast & 100 & 104.90 & 101.23 & 112.57 & 127.54 & 130.73 & 135.95 \\
Bryansk Oblast & 100 & 94.90 & 95.56 & 91.65 & 105.76 & 110.52 & 116.49 \\
Vladimir Oblast & 100 & 100.80 & 95.46 & 102.24 & 114.61 & 117.36 & 121.46
\end{tabular}




\begin{tabular}{|c|c|c|c|c|c|c|c|}
\hline 1 & 2 & 3 & 4 & 5 & 6 & 7 & 8 \\
\hline Volgograd Oblast & 100 & 103.00 & 90.54 & 91.62 & 102.44 & 112.58 & 117.19 \\
\hline Vologda Oblast & 100 & 99.20 & 94.93 & 108.23 & 116.34 & 118.55 & 121.52 \\
\hline Voronezh Oblast & 100 & 104.80 & 96.73 & 103.69 & 112.51 & 113.30 & 114.88 \\
\hline the city of Moscow & 100 & 111.10 & 103.55 & 110.69 & 131.61 & 135.69 & 149.39 \\
\hline $\begin{array}{l}\text { the city of Saint- } \\
\text { Petersburg }\end{array}$ & 100 & 98.60 & 93.37 & 99.16 & 109.58 & 114.51 & 134.77 \\
\hline $\begin{array}{l}\text { Jewish Autonomous } \\
\text { Oblast }\end{array}$ & 100 & 86.20 & 74.91 & 78.73 & 80.93 & 87.81 & 95.98 \\
\hline Ivanovo Oblast & 100 & 96.50 & 83.09 & 87.24 & 98.15 & 99.23 & 101.51 \\
\hline Irkutsk Oblast & 100 & 89.10 & 80.72 & 81.53 & 85.85 & 88.26 & 89.40 \\
\hline $\begin{array}{l}\text { Kabardino-Balkar } \\
\text { Republic }\end{array}$ & 100 & 97.60 & 98.09 & 106.92 & 126.59 & 145.20 & 153.18 \\
\hline Kaliningrad Oblast & 100 & 95.50 & 86.43 & 92.30 & 106.33 & 109.95 & 120.40 \\
\hline Kaluga Oblast & 100 & 93.50 & 88.92 & 90.25 & 97.20 & 104.98 & 107.60 \\
\hline Kamchatka Oblast & 100 & 97.60 & 89.79 & 83.51 & 88.77 & 85.13 & 82.06 \\
\hline $\begin{array}{l}\text { Karachay-Cherkessya } \\
\text { Republic }\end{array}$ & 100 & 97.80 & 97.80 & 95.06 & 106.85 & 120.85 & 129.91 \\
\hline Kemerovo Oblast & 100 & 96.20 & 88.41 & 95.75 & 102.35 & 107.06 & 111.02 \\
\hline Kirov Oblast & 100 & 96.60 & 91.00 & 95.27 & 102.13 & 101.11 & 100.61 \\
\hline Kostroma Oblast & 100 & 101.40 & 96.23 & 101.52 & 108.22 & 109.95 & 112.15 \\
\hline KrasnodarKrai & 100 & 94.20 & 89.21 & 104.02 & 115.04 & 112.97 & 122.57 \\
\hline Krasnoyarsk Krai & 100 & 100.20 & 93.79 & 98.57 & 103.60 & 110.12 & 114.53 \\
\hline Kurgan Oblast & 100 & 106.00 & 95.72 & 98.59 & 105.49 & 106.55 & 108.46 \\
\hline Kursk Oblast & 100 & 101.90 & 103.84 & 104.87 & 116.62 & 114.52 & 123.34 \\
\hline Leningrad Oblast & 100 & 97.10 & 90.98 & 103.45 & 116.69 & 126.61 & 147.24 \\
\hline Lipetsk Oblast & 100 & 94.50 & 90.44 & 93.33 & 101.08 & 104.51 & 115.49 \\
\hline Magadan Oblast & 100 & 97.00 & 78.67 & 76.31 & 77.68 & 79.62 & 83.76 \\
\hline Moscow Oblast & 100 & 108.20 & 105.60 & 111.09 & 118.54 & 126.95 & 133.30 \\
\hline Murmansk Oblast & 100 & 104.70 & 100.62 & 106.75 & 111.24 & 113.02 & 110.76 \\
\hline $\begin{array}{l}\text { Nizhny Novgorod } \\
\text { Oblast }\end{array}$ & 100 & 102.20 & 97.60 & 100.92 & 110.81 & 120.89 & 127.06 \\
\hline Novgorod Oblast & 100 & 97.10 & 102.63 & 109.31 & 112.48 & 126.20 & 123.93 \\
\hline Novosibirsk Oblast & 100 & 94.60 & 89.68 & 93.90 & 106.76 & 119.04 & 125.35 \\
\hline Omsk Oblast & 100 & 96.30 & 84.07 & 84.74 & 91.44 & 102.96 & 111.19 \\
\hline Orenburg Oblast & 100 & 105.70 & 91.22 & 99.43 & 109.07 & 110.16 & 115.67 \\
\hline Orel Oblast & 100 & 102.00 & 109.24 & 110.55 & 129.35 & 138.01 & 143.95 \\
\hline Penza Oblast & 100 & 101.70 & 89.60 & 100.44 & 106.57 & 112.11 & 114.13 \\
\hline Perm Oblast & 100 & 107.90 & 100.89 & 108.35 & 122.55 & 132.47 & 126.11 \\
\hline
\end{tabular}




\begin{tabular}{|c|c|c|c|c|c|c|c|}
\hline 1 & 2 & 3 & 4 & 5 & 6 & 7 & 8 \\
\hline Primorsky Krai & 100 & 99.70 & 93.62 & 99.70 & 98.01 & 97.91 & 102.41 \\
\hline Pskov Oblast & 100 & 88.70 & 82.14 & 96.59 & 102.29 & 102.50 & 107.72 \\
\hline Republic Adygeya & 100 & 90.90 & 87.72 & 92.46 & 97.91 & 98.69 & 99.68 \\
\hline Altay Republic & 100 & 95.60 & 86.42 & 90.31 & 98.80 & 118.17 & 113.68 \\
\hline $\begin{array}{l}\text { Republic Bashkor- } \\
\text { tostan }\end{array}$ & 100 & 100.70 & 92.04 & 96.09 & 101.47 & 109.69 & 112.65 \\
\hline Republic Buryatia & 100 & 102.20 & 96.17 & 103.86 & 108.85 & 115.82 & 123.23 \\
\hline Republic of Dagestan & 100 & 100.90 & 92.73 & 94.40 & 108.56 & 129.18 & 143.52 \\
\hline $\begin{array}{l}\text { Republic of Ingou- } \\
\text { shetia }\end{array}$ & 100 & 109.10 & 85.53 & 80.49 & 101.50 & 125.25 & 96.56 \\
\hline Republic of Kalmykia & 100 & 103.90 & 93.93 & 88.29 & 159.10 & 155.28 & 146.27 \\
\hline Republic Karelia & 100 & 94.20 & 87.42 & 96.95 & 104.99 & 107.93 & 116.03 \\
\hline Republic Komi & 100 & 98.40 & 97.71 & 94.10 & 103.51 & 111.79 & 108.10 \\
\hline Republic Mary-El & 100 & 101.90 & 97.42 & 97.61 & 94.88 & 98.77 & 97.78 \\
\hline Republic Mordovia & 100 & 104.90 & 100.28 & 101.99 & 111.98 & 119.82 & 130.13 \\
\hline $\begin{array}{l}\text { Republic of Sakha } \\
\text { (Yakutia) }\end{array}$ & 100 & 96.30 & 90.62 & 96.60 & 104.13 & 106.01 & 107.07 \\
\hline $\begin{array}{l}\text { Republic North } \\
\text { Ossetia (Alania) }\end{array}$ & 100 & 94.60 & 96.59 & 108.18 & 122.46 & 134.58 & 150.73 \\
\hline Republic Tatarstan & 100 & 101.00 & 92.72 & 99.76 & 106.75 & 118.38 & 122.53 \\
\hline Republic of Tyva & 100 & 99.50 & 95.32 & 101.61 & 104.76 & 119.43 & 123.73 \\
\hline Republic Khakassia & 100 & 100.30 & 91.57 & 90.84 & 97.93 & 101.55 & 98.30 \\
\hline Russian Federation & 100 & 101.20 & 94.72 & 100.03 & 110.73 & 117.37 & 123.95 \\
\hline Rostov Oblast & 100 & 101.30 & 97.96 & 107.26 & 119.28 & 138.48 & 144.30 \\
\hline Ryazan Oblast & 100 & 100.00 & 96.50 & 98.62 & 111.54 & 116.23 & 116.46 \\
\hline Samara Oblast & 100 & 105.40 & 97.50 & 99.93 & 106.33 & 113.56 & 117.76 \\
\hline Saratov Oblast & 100 & 112.00 & 98.45 & 104.45 & 118.24 & 122.02 & 128.49 \\
\hline Sakhalin Oblast & 100 & 99.10 & 95.04 & 113.19 & 95.98 & 111.92 & 118.86 \\
\hline Sverdlovsk Oblast & 100 & 96.90 & 89.15 & 90.75 & 101.82 & 110.68 & 115.33 \\
\hline Smolensk Oblast & 100 & 99.70 & 91.42 & 114.74 & 118.98 & 126.72 & 127.98 \\
\hline Stavropol Krai & 100 & 96.00 & 90.82 & 94.54 & 100.68 & 110.85 & 118.06 \\
\hline Tambov Oblast & 100 & 101.30 & 100.39 & 109.52 & 122.67 & 134.32 & 138.48 \\
\hline Tver Oblast & 100 & 91.70 & 93.53 & 98.12 & 104.59 & 107.52 & 109.46 \\
\hline Tomsk Oblast & 100 & 94.90 & 87.59 & 91.18 & 100.30 & 111.14 & 121.36 \\
\hline Tula Oblast & 100 & 93.40 & 90.32 & 90.05 & 99.50 & 102.98 & 105.15 \\
\hline Tyumen Oblast & 100 & 101.00 & 98.27 & 100.14 & 109.65 & 121.06 & 126.99 \\
\hline Udmurtia Republic & 100 & 102.60 & 95.93 & 97.27 & 110.21 & 116.38 & 115.34 \\
\hline
\end{tabular}




\begin{tabular}{lccccccc}
\hline \multicolumn{1}{c}{1} & 2 & 3 & 4 & 5 & 6 & 7 & 8 \\
\hline Ulyanovsk Oblast & 100 & 102.60 & 93.67 & 99.29 & 103.66 & 103.46 & 111.42 \\
Khabarovsk Krai & 100 & 104.50 & 97.92 & 106.92 & 119.33 & 129.11 & 137.38 \\
Chelyabinsk Oblast & 100 & 103.90 & 86.34 & 93.51 & 103.61 & 106.82 & 107.67 \\
Chita Oblast & 100 & 92.30 & 80.12 & 87.97 & 96.15 & 103.36 & 105.01 \\
Chuvash Republic & 100 & 95.00 & 86.36 & 86.96 & 92.44 & 98.26 & 100.91 \\
Chukotka autono- & 100 & 90.80 & 70.01 & 64.69 & 67.99 & 87.97 & 124.75 \\
mous okrug & 100 & 107.20 & 96.69 & 108.01 & 116.65 & 128.20 & 132.56
\end{tabular}

Table 2A-5

Weight Coefficients of Labor Input for the Regions

\begin{tabular}{|c|c|c|c|c|c|c|c|c|c|}
\hline Region & 1995 & 1996 & 1997 & 1998 & 1999 & 2000 & 2001 & 2002 & $\begin{array}{c}\text { Increase of coef- } \\
\text { ficient in } 2002 \text { as } \\
\% \text { of the } 1996 \\
\text { level }\end{array}$ \\
\hline 1 & 2 & 3 & 4 & 5 & 6 & 7 & 8 & 9 & 10 \\
\hline Russian Federation & 0.49 & 0.55 & 0.56 & 0.53 & 0.47 & 0.47 & 0.53 & 0.53 & -2.69 \\
\hline Altay Krai & 0.55 & 0.62 & 0.69 & 0.65 & 0.64 & 0.64 & 0.62 & 0.66 & 5.72 \\
\hline Amour Oblast & 0.77 & 0.67 & 0.65 & 0.65 & 0.66 & 0.75 & 0.68 & 0.78 & 15.89 \\
\hline Arkhangel' Oblast & 0.61 & 0.67 & 0.59 & 0.56 & 0.56 & 0.52 & 0.64 & 0.64 & -3.99 \\
\hline Astrakhan Oblast & 0.61 & 0.64 & 0.64 & 0.58 & 0.59 & 0.52 & 0.60 & 0.60 & -6.17 \\
\hline Belgorod Oblast & 0.42 & 0.53 & 0.53 & 0.50 & 0.47 & 0.53 & 0.62 & 0.63 & 20.39 \\
\hline Bryansk Oblast & 0.48 & 0.47 & 0.54 & 0.54 & 0.56 & 0.56 & 0.65 & 0.67 & 42.75 \\
\hline Vladimir Oblast & 0.50 & 0.61 & 0.64 & 0.63 & 0.60 & 0.67 & 0.71 & 0.76 & 25.52 \\
\hline Volgograd Oblast & 0.54 & 0.55 & 0.55 & 0.53 & 0.54 & 0.55 & 0.61 & 0.59 & 7.17 \\
\hline Vologda Oblast & 0.51 & 0.86 & 1.03 & 0.93 & 0.72 & 0.47 & 0.63 & 0.60 & -29.77 \\
\hline Voronezh Oblast & 0.41 & 0.51 & 0.52 & 0.53 & 0.54 & 0.58 & 0.62 & 0.57 & 9.94 \\
\hline the city of Moscow & 0.47 & 0.43 & 0.41 & 0.41 & 0.32 & 0.27 & 0.33 & 0.31 & -27.19 \\
\hline $\begin{array}{l}\text { the city of Saint- } \\
\text { Petersburg }\end{array}$ & 0.49 & 0.56 & 0.65 & 0.56 & 0.52 & 0.60 & 0.62 & 0.62 & 11.17 \\
\hline $\begin{array}{l}\text { Jewish Autonomous } \\
\text { Oblast }\end{array}$ & 0.73 & 0.85 & 0.76 & 0.95 & 0.72 & 0.79 & 0.85 & 0.86 & 2.16 \\
\hline Ivanovo Oblast & 0.54 & 0.60 & 0.70 & 0.65 & 0.65 & 0.66 & 0.72 & 0.75 & 26.49 \\
\hline Irkutsk Oblast & 0.55 & 0.58 & 0.56 & 0.55 & 0.55 & 0.60 & 0.66 & 0.69 & 18.64 \\
\hline $\begin{array}{l}\text { Kabardino-Balkar } \\
\text { Republic }\end{array}$ & 0.52 & 0.51 & 0.52 & 0.43 & 0.45 & 0.47 & 0.45 & 0.52 & 0.73 \\
\hline Kaliningrad Oblast & 0.62 & 0.65 & 0.73 & 0.74 & 0.61 & 0.60 & 0.60 & 0.66 & 0.79 \\
\hline
\end{tabular}




\begin{tabular}{|c|c|c|c|c|c|c|c|c|c|}
\hline 1 & 2 & 3 & 4 & 5 & 6 & 7 & 8 & 9 & 10 \\
\hline Kaluga Oblast & 0.47 & 0.61 & 0.66 & 0.67 & 0.63 & 0.65 & 0.68 & 0.73 & 20.99 \\
\hline Kamchatka Oblast & 0.52 & 0.58 & 0.63 & 0.46 & 0.48 & 0.56 & 0.59 & 0.75 & 28.97 \\
\hline $\begin{array}{l}\text { Karachay-Cherkessya } \\
\text { Republic }\end{array}$ & 0.47 & 0.52 & 0.57 & 0.54 & 0.49 & 0.57 & 0.59 & 0.61 & 17.13 \\
\hline Kemerovo Oblast & 0.55 & 0.62 & 0.64 & 0.64 & 0.62 & 0.66 & 0.69 & 0.65 & 5.51 \\
\hline Kirov Oblast & 0.44 & 0.54 & 0.54 & 0.57 & 0.58 & 0.60 & 0.68 & 0.70 & 29.38 \\
\hline Kostroma Oblast & 0.49 & 0.58 & 0.54 & 0.54 & 0.53 & 0.59 & 0.60 & 0.65 & 11.70 \\
\hline KrasnodarKrai & 0.47 & 0.50 & 0.58 & 0.53 & 0.45 & 0.48 & 0.52 & 0.54 & 8.15 \\
\hline Krasnoyarsk Krai & 0.53 & 0.63 & 0.62 & 0.55 & 0.47 & 0.48 & 0.56 & 0.67 & 5.24 \\
\hline Kurgan Oblast & 0.52 & 0.64 & 0.69 & 0.62 & 0.61 & 0.70 & 0.67 & 0.72 & 11.74 \\
\hline Kursk Oblast & 0.41 & 0.48 & 0.51 & 0.48 & 0.53 & 0.57 & 0.61 & 0.66 & 38.17 \\
\hline Leningrad Oblast & 0.49 & 0.57 & 0.62 & 0.55 & 0.48 & 0.53 & 0.55 & 0.56 & -2.30 \\
\hline Lipetsk Oblast & 0.36 & 0.49 & 0.55 & 0.52 & 0.43 & 0.46 & 0.56 & 0.48 & -2.27 \\
\hline Magadan Oblast & 0.99 & 0.83 & 0.79 & 0.72 & 0.72 & 0.77 & 0.77 & 0.69 & -17.13 \\
\hline Moscow Oblast & 0.47 & 0.51 & 0.56 & 0.46 & 0.48 & 0.57 & 0.63 & 0.57 & 12.67 \\
\hline Murmansk Oblast & 0.62 & 0.77 & 0.79 & 0.59 & 0.54 & 0.58 & 0.79 & 0.82 & 6.92 \\
\hline $\begin{array}{l}\text { Nizhny Novgorod } \\
\text { Oblast }\end{array}$ & 0.44 & 0.53 & 0.51 & 0.50 & 0.54 & 0.52 & 0.50 & 0.52 & -2.52 \\
\hline Novgorod Oblast & 0.61 & 0.61 & 0.64 & 0.53 & 0.47 & 0.53 & 0.54 & 0.60 & -0.63 \\
\hline Novosibirsk Oblast & 0.45 & 0.54 & 0.53 & 0.55 & 0.51 & 0.55 & 0.55 & 0.57 & 6.19 \\
\hline Omsk Oblast & 0.39 & 0.49 & 0.53 & 0.56 & 0.57 & 0.61 & 0.65 & 0.65 & 34.39 \\
\hline Orenburg Oblast & 0.50 & 0.51 & 0.50 & 0.53 & 0.45 & 0.48 & 0.54 & 0.56 & 9.79 \\
\hline Orel Oblast & 0.45 & 0.52 & 0.56 & 0.49 & 0.49 & 0.52 & 0.59 & 0.58 & 9.98 \\
\hline Penza Oblast & 0.52 & 0.53 & 0.60 & 0.64 & 0.62 & 0.64 & 0.65 & 0.67 & 25.97 \\
\hline Perm Oblast & 0.38 & 0.50 & 0.55 & 0.50 & 0.46 & 0.51 & 0.51 & 0.55 & 9.71 \\
\hline Primorsky Krai & 0.72 & 0.79 & 0.80 & 0.70 & 0.62 & 0.72 & 0.85 & 0.78 & -1.36 \\
\hline Pskov Oblast & 0.49 & 0.62 & 0.61 & 0.64 & 0.57 & 0.60 & 0.66 & 0.71 & 13.91 \\
\hline Republic Adygeya & 0.55 & 0.65 & 0.64 & 0.57 & 0.60 & 0.70 & 0.80 & 0.86 & 31.73 \\
\hline Altay Republic & 0.64 & 0.70 & 0.67 & 0.66 & 0.58 & 0.58 & 0.53 & 0.70 & -0.05 \\
\hline $\begin{array}{l}\text { Republic Bashkor- } \\
\text { tostan }\end{array}$ & 0.41 & 0.43 & 0.43 & 0.44 & 0.38 & 0.43 & 0.52 & 0.54 & 26.29 \\
\hline Republic Buryatia & 0.61 & 0.75 & 0.68 & 0.62 & 0.64 & 0.72 & 0.69 & 0.71 & -6.49 \\
\hline Republic of Dagestan & 0.60 & 0.67 & 0.61 & 0.61 & 0.57 & 0.63 & 0.53 & 0.60 & -11.06 \\
\hline $\begin{array}{l}\text { Republic of Ingou- } \\
\text { shetia }\end{array}$ & 0.45 & 0.49 & 0.47 & 0.55 & 0.38 & 0.26 & 0.48 & 0.87 & 77.78 \\
\hline Republic of Kalmykia & 0.78 & 0.87 & 0.82 & 0.80 & 0.84 & 0.34 & 0.35 & 0.39 & -55.03 \\
\hline Republic Karelia & 0.62 & 0.79 & 0.75 & 0.65 & 0.60 & 0.64 & 0.67 & 0.68 & -13.86 \\
\hline
\end{tabular}




\begin{tabular}{|c|c|c|c|c|c|c|c|c|c|}
\hline 1 & 2 & 3 & 4 & 5 & 6 & 7 & 8 & 9 & 10 \\
\hline Republic Komi & 0.55 & 0.71 & 0.62 & 0.55 & 0.51 & 0.56 & 0.59 & 0.63 & -10.73 \\
\hline Republic Mary-El & 0.46 & 0.59 & 0.54 & 0.55 & 0.59 & 0.66 & 0.66 & 0.75 & 26.08 \\
\hline Republic Mordovia & 0.49 & 0.50 & 0.51 & 0.47 & 0.45 & 0.39 & 0.52 & 0.72 & 44.58 \\
\hline $\begin{array}{l}\text { Republic of Sakha } \\
\text { (Yakutia) }\end{array}$ & 0.68 & 0.76 & 0.76 & 0.66 & 0.50 & 0.54 & 0.55 & 0.60 & -21.70 \\
\hline $\begin{array}{l}\text { Republic North } \\
\text { Ossetia (Alania) }\end{array}$ & 0.53 & 0.61 & 0.66 & 0.60 & 0.48 & 0.54 & 0.54 & 0.65 & 6.04 \\
\hline Republic Tatarstan & 0.42 & 0.42 & 0.45 & 0.41 & 0.37 & 0.34 & 0.44 & 0.43 & 2.15 \\
\hline Republic of Tyva & 0.58 & 0.66 & 0.65 & 0.64 & 0.51 & 0.52 & 0.57 & 0.67 & 1.69 \\
\hline Republic Khakassia & 0.56 & 0.69 & 0.61 & 0.62 & 0.57 & 0.62 & 0.73 & 0.72 & 4.39 \\
\hline Rostov Oblast & 0.53 & 0.62 & 0.62 & 0.54 & 0.49 & 0.54 & 0.61 & 0.68 & 9.95 \\
\hline Ryazan Oblast & 0.44 & 0.49 & 0.46 & 0.49 & 0.48 & 0.51 & 0.52 & 0.56 & 13.95 \\
\hline Samara Oblast & 0.41 & 0.47 & 0.43 & 0.44 & 0.40 & 0.43 & 0.45 & 0.49 & 4.97 \\
\hline Saratov Oblast & 0.37 & 0.48 & 0.47 & 0.52 & 0.49 & 0.50 & 0.50 & 0.55 & 14.75 \\
\hline Sakhalin Oblast & 0.81 & 0.88 & 0.79 & 0.75 & 0.54 & 0.60 & 0.57 & 0.62 & -29.68 \\
\hline Sverdlovsk Oblast & 0.39 & 0.54 & 0.55 & 0.52 & 0.51 & 0.57 & 0.61 & 0.66 & 22.20 \\
\hline Smolensk Oblast & 0.41 & 0.50 & 0.54 & 0.55 & 0.49 & 0.54 & 0.59 & 0.60 & 21.19 \\
\hline Stavropol Krai & 0.39 & 0.50 & 0.50 & 0.43 & 0.48 & 0.52 & 0.58 & 0.61 & 23.21 \\
\hline Tambov Oblast & 0.51 & 0.61 & 0.63 & 0.53 & 0.52 & 0.54 & 0.53 & 0.59 & -2.95 \\
\hline Tver Oblast & 0.50 & 0.55 & 0.57 & 0.52 & 0.52 & 0.57 & 0.59 & 0.67 & 21.59 \\
\hline Tomsk Oblast & 0.42 & 0.53 & 0.53 & 0.49 & 0.53 & 0.56 & 0.59 & 0.54 & 1.93 \\
\hline Tula Oblast & 0.52 & 0.63 & 0.67 & 0.60 & 0.57 & 0.61 & 0.66 & 0.66 & 4.36 \\
\hline Tyumen Oblast & 0.40 & 0.41 & 0.43 & 0.44 & 0.41 & 0.38 & 0.42 & 0.41 & -0.66 \\
\hline Udmurtia Republic & 0.44 & 0.53 & 0.57 & 0.56 & 0.46 & 0.49 & 0.57 & 0.57 & 8.52 \\
\hline Ulyanovsk Oblast & 0.44 & 0.46 & 0.52 & 0.52 & 0.48 & 0.55 & 0.59 & 0.64 & 40.39 \\
\hline Khabarovsk Krai & 0.67 & 0.60 & 0.56 & 0.60 & 0.61 & 0.59 & 0.66 & 0.70 & 17.72 \\
\hline Chelyabinsk Oblast & 0.48 & 0.51 & 0.56 & 0.63 & 0.52 & 0.50 & 0.59 & 0.58 & 13.57 \\
\hline Chita Oblast & 0.54 & 0.63 & 0.75 & 0.62 & 0.60 & 0.64 & 0.71 & 0.78 & 23.91 \\
\hline Chuvash Republic & 0.43 & 0.50 & 0.55 & 0.53 & 0.56 & 0.60 & 0.61 & 0.67 & 33.04 \\
\hline $\begin{array}{l}\text { Chukotka autono- } \\
\text { mous okrug }\end{array}$ & 0.68 & 0.58 & 0.66 & 0.55 & 0.58 & 0.56 & 0.43 & 0.44 & -24.19 \\
\hline Yaroslavl Oblast & 0.37 & 0.48 & 0.52 & 0.48 & 0.46 & 0.56 & 0.51 & 0.53 & 11.39 \\
\hline
\end{tabular}


Table 2A-6

\section{Results of the Assessment of Growth Rates of the Components of Labor Reserves across the Regions}

\begin{tabular}{|c|c|c|c|c|c|c|c|c|c|}
\hline \multirow[b]{2}{*}{ Region } & \multicolumn{3}{|c|}{ 1996-1998 } & \multicolumn{3}{|c|}{ 1999-2002 } & \multicolumn{3}{|c|}{ 1996-2002 } \\
\hline & $\begin{array}{l}\text { Number } \\
\text { of em- } \\
\text { ployed }\end{array}$ & $\begin{array}{l}\text { Structure } \\
\text { of em- } \\
\text { ployed }\end{array}$ & $\begin{array}{l}\text { Labor } \\
\text { input }\end{array}$ & $\begin{array}{c}\text { Number } \\
\text { of em- } \\
\text { ployed }\end{array}$ & $\begin{array}{l}\text { Structure } \\
\text { of em- } \\
\text { ployed }\end{array}$ & $\begin{array}{c}\text { Labor } \\
\text { input }\end{array}$ & $\begin{array}{l}\text { Number } \\
\text { of em- } \\
\text { ployed }\end{array}$ & $\begin{array}{l}\text { Structure } \\
\text { of em- } \\
\text { ployed }\end{array}$ & $\begin{array}{l}\text { Labor } \\
\text { input }\end{array}$ \\
\hline 1 & 2 & 3 & 4 & 5 & 6 & 7 & 8 & 9 & 10 \\
\hline Altay Krai & -1.28 & 0.00 & -1.28 & 0.68 & -0.64 & 0.06 & 0.03 & -0.43 & -0.39 \\
\hline Amour Oblast & -2.08 & -1.33 & -3.43 & 0.33 & -0.36 & -0.02 & -0.48 & -0.69 & -1.17 \\
\hline Arkhangel' Oblast & -3.18 & 0.00 & -3.19 & 1.22 & -0.37 & 0.85 & -0.27 & -0.25 & -0.52 \\
\hline Astrakhan Oblast & -2.35 & -0.97 & -3.32 & 1.10 & -0.95 & 0.15 & -0.07 & -0.96 & -1.02 \\
\hline Belgorod Oblast & -1.63 & 0.07 & -1.56 & 1.22 & -0.59 & 0.66 & 0.27 & -0.37 & -0.09 \\
\hline Bryansk Oblast & -1.72 & -0.17 & -1.88 & 1.33 & -1.11 & 0.24 & 0.31 & -0.79 & -0.47 \\
\hline Vladimir Oblast & -0.11 & -0.72 & -0.83 & 0.49 & 0.01 & 0.50 & 0.29 & -0.23 & 0.06 \\
\hline Volgograd Oblast & -3.43 & -0.37 & -3.80 & 1.48 & -1.04 & 0.45 & -0.18 & -0.82 & -0.99 \\
\hline Vologda Oblast & -2.45 & 0.67 & -1.78 & 1.61 & -0.35 & 1.28 & 0.24 & -0.01 & 0.25 \\
\hline Voronezh Oblast & -1.38 & -0.43 & -1.81 & 1.28 & -0.50 & 0.80 & 0.38 & -0.48 & -0.08 \\
\hline the city of Moscow & 0.39 & -0.12 & 0.27 & 0.43 & -0.11 & 0.32 & 0.42 & -0.12 & 0.30 \\
\hline $\begin{array}{l}\text { the city of Saint- } \\
\text { Petersburg }\end{array}$ & -0.02 & 0.62 & 0.60 & 0.31 & 0.06 & 0.37 & 0.20 & 0.24 & 0.45 \\
\hline $\begin{array}{l}\text { Jewish Autonomous } \\
\text { Oblast }\end{array}$ & -4.84 & 0.07 & -4.76 & 2.27 & -1.45 & 0.87 & -0.16 & -0.95 & -1.04 \\
\hline Ivanovo Oblast & -2.53 & 0.22 & -2.29 & 0.10 & -0.48 & -0.37 & -0.78 & -0.25 & -1.01 \\
\hline Irkutsk Oblast & -1.13 & -0.78 & -1.91 & 0.67 & -0.51 & 0.16 & 0.07 & -0.60 & -0.54 \\
\hline $\begin{array}{l}\text { Kabardino-Balkar } \\
\text { Republic }\end{array}$ & -1.23 & -0.05 & -1.28 & 2.39 & -0.68 & 1.72 & 1.17 & -0.47 & 0.71 \\
\hline Kaliningrad Oblast & 0.13 & -0.25 & -0.13 & 0.42 & -0.51 & -0.09 & 0.32 & -0.42 & -0.11 \\
\hline Kaluga Oblast & -2.12 & 0.48 & -1.64 & 0.29 & -0.67 & -0.37 & -0.52 & -0.29 & -0.80 \\
\hline Kamchatka Oblast & -2.32 & 0.02 & -2.29 & -0.06 & -0.46 & -0.53 & -0.82 & -0.30 & -1.12 \\
\hline $\begin{array}{l}\text { Karachay-Cherkessya } \\
\text { Republic }\end{array}$ & -1.54 & -0.66 & -2.19 & 1.57 & -0.73 & 0.85 & 0.52 & -0.70 & -0.17 \\
\hline Kemerovo Oblast & -2.27 & -0.20 & -2.47 & 0.01 & -0.30 & -0.29 & -0.76 & -0.26 & -1.02 \\
\hline Kirov Oblast & -0.76 & 0.02 & -0.74 & 0.86 & -0.59 & 0.29 & 0.31 & -0.39 & -0.06 \\
\hline Kostroma Oblast & -1.66 & -0.11 & -1.77 & 0.15 & -0.18 & -0.03 & -0.46 & -0.16 & -0.61 \\
\hline KrasnodarKrai & -1.18 & -0.02 & -1.20 & 1.49 & -0.35 & 1.15 & 0.59 & -0.24 & 0.36 \\
\hline Krasnoyarsk Krai & -0.77 & -0.40 & -1.17 & 0.61 & -0.56 & 0.05 & 0.15 & -0.50 & -0.36 \\
\hline Kurgan Oblast & -2.98 & 0.24 & -2.75 & 0.95 & -1.05 & -0.08 & -0.38 & -0.62 & -0.98 \\
\hline
\end{tabular}




\begin{tabular}{|c|c|c|c|c|c|c|c|c|c|}
\hline 1 & 2 & 3 & 4 & 5 & 6 & 7 & 8 & 9 & 10 \\
\hline Kursk Oblast & 0.13 & -0.11 & 0.02 & 0.91 & -0.81 & 0.11 & 0.65 & -0.58 & 0.08 \\
\hline Leningrad Oblast & -0.83 & 0.27 & -0.56 & 0.66 & 0.17 & 0.83 & 0.16 & 0.20 & 0.37 \\
\hline Lipetsk Oblast & -1.37 & 0.03 & -1.35 & 1.25 & -0.44 & 0.83 & 0.37 & -0.28 & 0.10 \\
\hline Magadan Oblast & -4.06 & -0.12 & -4.28 & -1.34 & -0.25 & -1.61 & -2.26 & -0.21 & -2.51 \\
\hline Moscow Oblast & -0.22 & -0.32 & -0.54 & 0.82 & -0.03 & 0.79 & 0.47 & -0.13 & 0.34 \\
\hline Murmansk Oblast & -2.36 & -1.11 & -3.46 & 0.49 & -0.21 & 0.28 & -0.47 & -0.51 & -0.98 \\
\hline $\begin{array}{l}\text { Nizhny Novgorod } \\
\text { Oblast }\end{array}$ & -1.30 & 0.45 & -0.84 & 0.42 & 0.00 & 0.42 & -0.15 & 0.15 & 0.00 \\
\hline Novgorod Oblast & -3.31 & 0.76 & -2.54 & 0.49 & 0.02 & 0.51 & -0.79 & 0.26 & -0.52 \\
\hline Novosibirsk Oblast & -2.21 & -0.17 & -2.39 & 0.88 & -0.37 & 0.51 & -0.16 & -0.31 & -0.46 \\
\hline Omsk Oblast & -0.41 & -0.54 & -0.95 & 0.47 & 0.58 & 1.06 & 0.18 & 0.21 & 0.38 \\
\hline Orenburg Oblast & -0.82 & -0.37 & -1.19 & 1.33 & -0.41 & 0.93 & 0.61 & -0.39 & 0.22 \\
\hline Orel Oblast & -0.96 & -0.22 & -1.17 & 1.33 & -0.48 & 0.86 & 0.56 & -0.39 & 0.18 \\
\hline Penza Oblast & -1.67 & -0.13 & -1.80 & 1.12 & -0.76 & 0.38 & 0.18 & -0.55 & -0.35 \\
\hline Perm Oblast & -1.58 & 0.01 & -1.57 & 1.28 & -0.07 & 1.21 & 0.32 & -0.04 & 0.27 \\
\hline Primorsky Krai & -1.34 & -0.33 & -1.67 & 1.08 & -0.41 & 0.68 & 0.27 & -0.38 & -0.11 \\
\hline Pskov Oblast & -2.15 & 0.13 & -2.01 & 1.58 & -0.81 & 0.84 & 0.32 & -0.50 & -0.12 \\
\hline Republic Adygeya & -2.50 & -1.83 & -4.34 & 0.58 & -0.31 & 0.28 & -0.46 & -0.82 & -1.29 \\
\hline Altay Republic & -3.33 & -0.84 & -4.17 & 1.58 & -0.76 & 0.87 & -0.08 & -0.79 & -0.84 \\
\hline $\begin{array}{l}\text { Republic Bashkor- } \\
\text { tostan }\end{array}$ & -0.83 & 0.15 & -0.68 & 0.62 & -0.49 & 0.13 & 0.13 & -0.28 & -0.14 \\
\hline Republic Buryatia & -5.30 & -0.36 & -5.67 & 0.98 & -1.04 & -0.04 & -1.16 & -0.81 & -1.95 \\
\hline Republic of Dagestan & 2.12 & -0.58 & 1.44 & 1.59 & -1.57 & -0.08 & 1.77 & -1.24 & 0.42 \\
\hline $\begin{array}{l}\text { Republic of Ingou- } \\
\text { shetia }\end{array}$ & -0.20 & -3.86 & -4.04 & 2.63 & -0.41 & 2.15 & 1.68 & -1.57 & 0.04 \\
\hline Republic of Kalmykia & -7.27 & 0.40 & -6.89 & 0.21 & -1.44 & -1.22 & -2.35 & -0.83 & -3.15 \\
\hline Republic Karelia & -5.46 & -0.79 & -6.26 & 1.48 & -0.02 & 1.46 & -0.89 & -0.28 & -1.18 \\
\hline Republic Komi & -1.69 & 0.19 & -1.48 & 0.45 & -0.70 & -0.25 & -0.27 & -0.40 & -0.66 \\
\hline Republic Mary-El & -1.50 & 0.15 & -1.35 & 0.69 & -0.58 & 0.15 & -0.04 & -0.34 & -0.35 \\
\hline Republic Mordovia & -0.40 & 0.00 & -0.40 & 0.34 & -0.17 & 0.16 & 0.09 & -0.12 & -0.02 \\
\hline $\begin{array}{l}\text { Republic of Sakha } \\
\text { (Yakutia) }\end{array}$ & -3.33 & 0.10 & -3.23 & -0.18 & -0.61 & -0.80 & -1.24 & -0.38 & -1.61 \\
\hline $\begin{array}{l}\text { Republic North } \\
\text { Ossetia (Alania) }\end{array}$ & -0.68 & -0.87 & -1.54 & 2.28 & -0.75 & 1.53 & 1.29 & -0.79 & 0.50 \\
\hline Republic Tatarstan & -0.81 & -0.08 & -0.89 & 0.69 & -0.14 & 0.56 & 0.19 & -0.12 & 0.07 \\
\hline Republic of Tyva & -3.58 & -0.28 & -3.87 & -0.89 & -0.31 & -1.20 & -1.79 & -0.30 & -2.09 \\
\hline Republic Khakassia & -0.97 & -0.37 & -1.34 & 0.99 & -0.82 & 0.17 & 0.33 & -0.67 & -0.34 \\
\hline Rostov Oblast & -1.75 & -1.04 & -2.79 & 1.07 & -0.41 & 0.66 & 0.12 & -0.62 & -0.50 \\
\hline
\end{tabular}




\begin{tabular}{|c|c|c|c|c|c|c|c|c|c|}
\hline 1 & 2 & 3 & 4 & 5 & 6 & 7 & 8 & 9 & 10 \\
\hline Ryazan Oblast & -1.87 & -0.16 & -2.02 & 0.84 & -0.38 & 0.47 & -0.07 & -0.30 & -0.37 \\
\hline Samara Oblast & -0.66 & -0.41 & -1.07 & 0.82 & -0.18 & 0.63 & 0.32 & -0.26 & 0.06 \\
\hline Saratov Oblast & -0.60 & -0.41 & -1.01 & 0.34 & -0.60 & -0.26 & 0.03 & -0.54 & -0.51 \\
\hline Sakhalin Oblast & -2.66 & -1.37 & -4.03 & 0.86 & 0.02 & 0.88 & -0.33 & -0.44 & -0.78 \\
\hline Sverdlovsk Oblast & -1.06 & 0.14 & -0.92 & 0.73 & -0.34 & 0.39 & 0.13 & -0.18 & -0.05 \\
\hline Smolensk Oblast & -1.51 & -0.41 & -1.92 & 0.99 & -0.23 & 0.76 & 0.15 & -0.29 & -0.14 \\
\hline Stavropol Krai & -1.25 & -0.50 & -1.75 & 0.74 & -0.37 & 0.37 & 0.08 & -0.41 & -0.34 \\
\hline Tambov Oblast & -3.01 & -0.01 & -3.02 & 1.00 & -0.83 & 0.21 & -0.36 & -0.55 & -0.88 \\
\hline Tver Oblast & -2.08 & -0.19 & -2.27 & 0.74 & -0.36 & 0.39 & -0.21 & -0.30 & -0.51 \\
\hline Tomsk Oblast & -2.02 & -0.10 & -2.12 & 0.70 & -0.01 & 0.69 & -0.22 & -0.04 & -0.25 \\
\hline Tula Oblast & -2.25 & -0.40 & -2.65 & 0.12 & -0.33 & -0.20 & -0.68 & -0.35 & -1.02 \\
\hline Tyumen Oblast & -1.43 & 0.60 & -0.83 & 0.91 & -0.11 & 0.80 & 0.12 & 0.12 & 0.25 \\
\hline Udmurtia Republic & -1.20 & -0.09 & -1.29 & 1.36 & -0.34 & 1.03 & 0.50 & -0.26 & 0.25 \\
\hline Ulyanovsk Oblast & -1.54 & -0.20 & -1.73 & 0.40 & -0.39 & 0.02 & -0.25 & -0.32 & -0.57 \\
\hline Khabarovsk Krai & -0.03 & -1.13 & -1.16 & 1.48 & -0.17 & 1.31 & 0.97 & -0.49 & 0.48 \\
\hline Chelyabinsk Oblast & -0.36 & -0.22 & -0.58 & 0.54 & -0.25 & 0.28 & 0.24 & -0.24 & 0.00 \\
\hline Chita Oblast & -3.87 & -0.86 & -4.78 & 1.53 & -1.10 & 0.43 & -0.31 & -1.02 & -1.34 \\
\hline Chuvash Republic & -1.11 & -0.13 & -1.24 & 1.36 & -0.62 & 0.76 & 0.53 & -0.45 & 0.09 \\
\hline $\begin{array}{l}\text { Chukotka autono- } \\
\text { mous okrug }\end{array}$ & -7.50 & -0.19 & -7.69 & -1.12 & 0.07 & -1.05 & -3.29 & -0.02 & -3.32 \\
\hline Yaroslavl Oblast & -0.93 & -0.27 & -1.20 & 0.78 & -0.20 & 0.58 & 0.20 & -0.22 & -0.02 \\
\hline
\end{tabular}

Table 2A-7

Fixed Assets Depreciation Rate (at the beginning of year; percent)

\begin{tabular}{lccccccccc}
\hline \multicolumn{1}{c}{ Region } & $\mathbf{1 9 9 9}$ & $\mathbf{2 0 0 0}$ & $\mathbf{2 0 0 1}$ & $\mathbf{2 0 0 2}$ & Region & $\mathbf{1 9 9 9}$ & $\mathbf{2 0 0 0}$ & $\mathbf{2 0 0 1}$ & $\mathbf{2 0 0 2}$ \\
\hline \multicolumn{1}{c}{1} & 2 & 3 & 4 & 5 & 6 & 7 & 8 & 9 & 10 \\
\hline Russian Federation & $\mathbf{4 1 . 9}$ & $\mathbf{4 2 . 4}$ & $\mathbf{4 5 . 8}$ & $\mathbf{4 7 . 9}$ & Perm Oblast & 46.1 & 47 & 51.3 & 51.6 \\
Altay Krai & 43.2 & 43.3 & 47 & 48.2 & Primorsky Krai & 37.7 & 40.6 & 43.2 & 45.2 \\
Amour Oblast & 31.1 & 36 & 41.4 & 42.5 & Pskov Oblast & 42 & 39.1 & 43.3 & 45.8 \\
Arkhangel' Oblast & 48.1 & 47.6 & 52.2 & 47.9 & Republic Adygeya & 48.9 & 48.4 & 50 & 51 \\
Astrakhan Oblast & 45 & 43.1 & 43.6 & 44.8 & Altay Republic & 40.6 & 37.7 & 35.8 & 36.4 \\
Belgorod Oblast & 39.3 & 39.4 & 41.3 & 45.3 & Republic Bashkor- & 46.2 & 44.8 & 45 & 46.7 \\
Bryansk Oblast & 45.8 & 47.3 & 49.6 & 52 & Republic Buryatia & 34.4 & 34.4 & 35 & 37.1 \\
Vladimir Oblast & 41.2 & 43 & 44.1 & 45.4 & Republic of Dagestan & 38.9 & 36.8 & 38.8 & 38.3
\end{tabular}




\begin{tabular}{|c|c|c|c|c|c|c|c|c|c|}
\hline 1 & 2 & 3 & 4 & 5 & 6 & 7 & 8 & 9 & 10 \\
\hline Volgograd Oblast & 46.8 & 50 & 50.8 & 52.8 & $\begin{array}{l}\text { Republic of Ingou- } \\
\text { shetia }\end{array}$ & 63.6 & 61.4 & 51.2 & 48.6 \\
\hline Vologda Oblast & 40.3 & 41.6 & 43.6 & 43.2 & Republic of Kalmykia & 39.3 & 40.5 & 36.9 & 33.2 \\
\hline Voronezh Oblast & 46.2 & 47.7 & 49.9 & 51.9 & Republic Karelia & 39.2 & 40.5 & 40.7 & 44.1 \\
\hline the city of Moscow & 37.7 & 39.2 & 48.4 & 50.1 & Republic Komi & 41.5 & 32.8 & 38.2 & 41.6 \\
\hline $\begin{array}{l}\text { the city of Saint- } \\
\text { Petersburg }\end{array}$ & 46.6 & 46 & 44.3 & 47 & Republic Mary-El & 38.2 & 38.2 & 38.9 & 39 \\
\hline $\begin{array}{l}\text { Jewish Autonomous } \\
\text { Oblast }\end{array}$ & 33.9 & 36 & 43.5 & 46 & Republic Mordovia & 40.9 & 43.1 & 45.2 & 50.5 \\
\hline Ivanovo Oblast & 40.9 & 43 & 44.3 & 45.2 & $\begin{array}{l}\text { Republic of Sakha } \\
\text { (Yakutia) }\end{array}$ & 37 & 37.5 & 39.3 & 42.7 \\
\hline Irkutsk Oblast & 40 & 41.5 & 44.7 & 47.7 & $\begin{array}{l}\text { Republic North } \\
\text { Ossetia (Alania) }\end{array}$ & 43.1 & 41.6 & 41.6 & 42.2 \\
\hline $\begin{array}{l}\text { Kabardino-Balkar } \\
\text { Republic }\end{array}$ & 41.5 & 38.7 & 36.4 & 45.1 & Republic Tatarstan & 42.6 & 42.1 & 40.8 & 45.3 \\
\hline Kaliningrad Oblast & 44.2 & 45.3 & 46.9 & 48.1 & Republic of Tyva & 35.5 & 40.1 & 43.4 & 48.5 \\
\hline Kaluga Oblast & 37.1 & 41.5 & 42.9 & 41.2 & Republic Khakassia & 36.5 & 30.3 & 38.4 & 43.7 \\
\hline Kamchatka Oblast & 29.1 & 30.7 & 31.3 & 36 & Rostov Oblast & 47.6 & 48.8 & 49.9 & 51.2 \\
\hline $\begin{array}{l}\text { Karachay-Cherkessya } \\
\text { Republic }\end{array}$ & 41.3 & 41.6 & 42.1 & 40.7 & Ryazan Oblast & 46.5 & 47 & 48.6 & 49.7 \\
\hline Kemerovo Oblast & 39.6 & 42.2 & 42.4 & 47.4 & Samara Oblast & 49.5 & 56.1 & 56 & 56.1 \\
\hline Kirov Oblast & 46.6 & 49.3 & 51.3 & 57 & Saratov Oblast & 43.3 & 45.8 & 48.1 & 50.2 \\
\hline Kostroma Oblast & 37.4 & 43.4 & 49.7 & 55.3 & Sakhalin Oblast & 37.2 & 37 & 36.4 & 40 \\
\hline KrasnodarKrai & 46 & 45.3 & 45.3 & 43 & Sverdlovsk Oblast & 43.9 & 45.4 & 48.7 & 51.9 \\
\hline Krasnoyarsk Krai & 38.6 & 39.4 & 38.4 & 42.2 & Smolensk Oblast & 40.7 & 44.1 & 46.4 & 50.3 \\
\hline Kurgan Oblast & 43.1 & 44 & 48.1 & 44.5 & Stavropol Krai & 43.1 & 44.2 & 45.9 & 47.7 \\
\hline Kursk Oblast & 47.2 & 49 & 51.7 & 53.2 & Tambov Oblast & 41.5 & 44.7 & 49.2 & 52.3 \\
\hline Leningrad Oblast & 39.7 & 40.9 & 40.5 & 41.7 & Tver Oblast & 41 & 42.7 & 48.7 & 51.3 \\
\hline Lipetsk Oblast & 45.7 & 47 & 48.1 & 48.5 & Tomsk Oblast & 48.1 & 46.8 & 48.1 & 47.4 \\
\hline Magadan Oblast & 35.9 & 39.2 & 42.5 & 45.3 & Tula Oblast & 45.6 & 46.9 & 48.2 & 50.5 \\
\hline Moscow Oblast & 37.5 & 35.5 & 37 & 38.5 & Tyumen Oblast & 44.4 & 38.1 & 38.7 & 44.3 \\
\hline Murmansk Oblast & 43.2 & 41.5 & 43.3 & 46.2 & Udmurtia Republic & 45.7 & 46.9 & 47.7 & 50.6 \\
\hline $\begin{array}{l}\text { Nizhny Novgorod } \\
\text { Oblast }\end{array}$ & 40.2 & 40.7 & 45.5 & 48 & Ulyanovsk Oblast & 36.1 & 37 & 40.5 & 43.6 \\
\hline Novgorod Oblast & 50.5 & 48.1 & 50 & 51.8 & Khabarovsk Krai & 32.7 & 37.2 & 42.5 & 44.7 \\
\hline Novosibirsk Oblast & 48.5 & 49 & 53.7 & 53.5 & Chelyabinsk Oblast & 43.4 & 45.5 & 47.2 & 48.5 \\
\hline Omsk Oblast & 45.7 & 46.7 & 48.5 & 50.7 & Chita Oblast & 41.3 & 46.3 & 45.8 & 49.5 \\
\hline Orenburg Oblast & 49.3 & 48.4 & 49 & 51.1 & Chuvash Republic & 39.9 & 37.5 & 42.1 & 37.7 \\
\hline Orel Oblast & 42 & 43.2 & 42.8 & 45.5 & $\begin{array}{l}\text { Chukotka autono- } \\
\text { mous okrug }\end{array}$ & 29.5 & 27.5 & 31.7 & 36.3 \\
\hline Penza Oblast & 42.2 & 48.8 & 51.5 & 53.7 & Yaroslavl Oblast & 46.7 & 52.6 & 56.9 & 57.2 \\
\hline
\end{tabular}


Table 2A-8

\section{Results of Assessment of the Growth rates of Components of Capital Input by Regions}

\begin{tabular}{|c|c|c|c|c|c|c|c|c|c|}
\hline \multirow[b]{2}{*}{ Region } & \multicolumn{3}{|c|}{ 1997-1998 } & \multicolumn{3}{|c|}{ 1999-2002 } & \multicolumn{3}{|c|}{ 1997-2002 } \\
\hline & $\begin{array}{l}\text { Change } \\
\text { in the } \\
\text { volume } \\
\text { of FA }\end{array}$ & $\begin{array}{l}\text { Change } \\
\text { of the } \\
\text { level of } \\
\text { capacity } \\
\text { loading }\end{array}$ & $\begin{array}{c}\text { Capital } \\
\text { input }\end{array}$ & $\begin{array}{c}\text { Change } \\
\text { in the } \\
\text { volume } \\
\text { of FA }\end{array}$ & $\begin{array}{c}\text { Change } \\
\text { of the } \\
\text { level of } \\
\text { capacity } \\
\text { loading }\end{array}$ & $\begin{array}{c}\text { Capital } \\
\text { input }\end{array}$ & $\begin{array}{l}\text { Change } \\
\text { in the } \\
\text { volume } \\
\text { of FA }\end{array}$ & $\begin{array}{l}\text { Change } \\
\text { of the } \\
\text { level of } \\
\text { capacity } \\
\text { loading }\end{array}$ & $\begin{array}{c}\text { Capital } \\
\text { input }\end{array}$ \\
\hline 1 & 2 & 3 & 4 & 5 & 6 & 7 & 8 & 9 & 10 \\
\hline Russian Federation & -0.02 & -1.26 & -1.28 & 0.19 & 1.06 & 1.24 & 0.12 & 0.28 & 0.40 \\
\hline Altay Krai & -0.22 & -1.68 & -1.90 & -0.15 & -1.30 & -1.45 & -0.17 & -1.42 & -1.60 \\
\hline Amour Oblast & -0.17 & -2.27 & -2.44 & -0.05 & 0.52 & 0.48 & -0.09 & -0.42 & -0.50 \\
\hline Arkhangel' Oblast & -0.21 & -0.67 & -0.88 & 0.03 & 0.61 & 0.64 & -0.05 & 0.19 & 0.13 \\
\hline Astrakhan Oblast & -0.09 & -0.21 & -0.29 & 0.44 & 0.60 & 1.04 & 0.26 & 0.33 & 0.59 \\
\hline Belgorod Oblast & 0.02 & -0.46 & -0.44 & 0.13 & 1.62 & 1.75 & 0.10 & 0.92 & 1.02 \\
\hline Bryansk Oblast & -0.33 & -2.17 & -2.50 & -0.19 & 1.28 & 1.09 & -0.24 & 0.12 & -0.12 \\
\hline Vladimir Oblast & -0.12 & -0.53 & -0.64 & -0.04 & 1.16 & 1.11 & -0.07 & 0.59 & 0.52 \\
\hline Volgograd Oblast & -0.15 & -5.76 & -5.91 & -0.07 & -0.34 & -0.42 & -0.10 & -2.18 & -2.28 \\
\hline Vologda Oblast & -0.01 & -0.06 & -0.07 & 0.01 & 0.56 & 0.57 & 0.00 & 0.35 & 0.36 \\
\hline Voronezh Oblast & -0.26 & -1.64 & -1.89 & -0.12 & 0.22 & 0.10 & -0.17 & -0.40 & -0.57 \\
\hline the city of Moscow & 0.77 & 1.05 & 1.82 & 1.23 & 2.76 & 3.99 & 1.07 & 2.19 & 3.26 \\
\hline $\begin{array}{l}\text { the city of Saint- } \\
\text { Petersburg }\end{array}$ & -0.05 & 0.05 & 0.00 & 0.31 & 1.96 & 2.26 & 0.19 & 1.32 & 1.50 \\
\hline $\begin{array}{l}\text { Jewish Autonomous } \\
\text { Oblast }\end{array}$ & -0.10 & -0.64 & -0.74 & -0.13 & 0.28 & 0.15 & -0.12 & -0.03 & -0.15 \\
\hline Ivanovo Oblast & -0.22 & -0.49 & -0.71 & -0.17 & 0.78 & 0.61 & -0.19 & 0.35 & 0.17 \\
\hline Irkutsk Oblast & -0.23 & -0.79 & -1.03 & -0.17 & 1.08 & 0.91 & -0.19 & 0.45 & 0.26 \\
\hline $\begin{array}{l}\text { Kabardino-Balkar } \\
\text { Republic }\end{array}$ & -0.07 & -1.70 & -1.77 & 0.18 & -1.42 & -1.25 & 0.09 & -1.51 & -1.42 \\
\hline Kaliningrad Oblast & -0.16 & -0.29 & -0.45 & 0.00 & 0.61 & 0.61 & -0.06 & 0.31 & 0.26 \\
\hline Kaluga Oblast & -0.10 & -0.50 & -0.59 & 0.03 & 0.94 & 0.97 & -0.02 & 0.46 & 0.44 \\
\hline Kamchatka Oblast & -0.24 & -0.92 & -1.16 & -0.14 & -1.21 & -1.35 & -0.17 & -1.12 & -1.29 \\
\hline $\begin{array}{l}\text { Karachay-Cherkessya } \\
\text { Republic }\end{array}$ & -0.14 & 0.79 & 0.65 & -0.11 & -0.31 & -0.42 & -0.12 & 0.06 & -0.07 \\
\hline Kemerovo Oblast & 0.10 & -1.03 & -0.92 & 0.05 & 0.64 & 0.69 & 0.07 & 0.08 & 0.15 \\
\hline Kirov Oblast & -0.28 & -0.43 & -0.72 & -0.15 & 0.46 & 0.32 & -0.19 & 0.16 & -0.03 \\
\hline Kostroma Oblast & -0.14 & 0.08 & -0.07 & 0.07 & -0.50 & -0.43 & 0.00 & -0.31 & -0.31 \\
\hline
\end{tabular}




\begin{tabular}{|c|c|c|c|c|c|c|c|c|c|}
\hline 1 & 2 & 3 & 4 & 5 & 6 & 7 & 8 & 9 & 10 \\
\hline KrasnodarKrai & 0.06 & -0.39 & -0.32 & 0.71 & 1.21 & 1.92 & 0.49 & 0.67 & 1.16 \\
\hline Krasnoyarsk Krai & -0.06 & -0.22 & -0.29 & 0.01 & -0.07 & -0.06 & -0.02 & -0.12 & -0.14 \\
\hline Kurgan Oblast & -0.20 & -1.18 & -1.38 & -0.16 & -0.43 & -0.58 & -0.17 & -0.68 & -0.85 \\
\hline Kursk Oblast & -0.28 & 0.70 & 0.42 & -0.15 & -0.37 & -0.52 & -0.19 & -0.01 & -0.20 \\
\hline Leningrad Oblast & -0.04 & - & -0.04 & 0.48 & - & 0.48 & 0.31 & - & 0.31 \\
\hline Lipetsk Oblast & -0.17 & -4.14 & -4.30 & -0.10 & 0.44 & 0.34 & -0.12 & -1.11 & -1.23 \\
\hline Magadan Oblast & -0.05 & -0.57 & -0.63 & -0.08 & -1.48 & -1.56 & -0.07 & -1.18 & -1.25 \\
\hline Moscow Oblast & 0.15 & - & 0.15 & 0.44 & - & 0.44 & 0.34 & - & 0.34 \\
\hline Murmansk Oblast & -0.19 & -0.37 & -0.55 & -0.09 & -0.17 & -0.26 & -0.12 & -0.24 & -0.36 \\
\hline $\begin{array}{l}\text { Nizhny Novgorod } \\
\text { Oblast }\end{array}$ & -0.01 & -1.82 & -1.82 & 0.00 & 0.48 & 0.48 & 0.00 & -0.29 & -0.30 \\
\hline Novgorod Oblast & -0.14 & -0.72 & -0.86 & 0.19 & -0.42 & -0.24 & 0.08 & -0.52 & -0.44 \\
\hline Novosibirsk Oblast & -0.16 & -2.91 & -3.07 & -0.09 & 0.34 & 0.25 & -0.11 & -0.76 & -0.87 \\
\hline Omsk Oblast & -0.19 & -2.69 & -2.88 & -0.13 & -0.30 & -0.43 & -0.15 & -1.10 & -1.25 \\
\hline Orenburg Oblast & -0.05 & -0.83 & -0.89 & 0.04 & 0.95 & 0.99 & 0.01 & 0.35 & 0.36 \\
\hline Orel Oblast & -0.20 & 0.51 & 0.31 & 0.02 & -0.66 & -0.64 & -0.06 & -0.27 & -0.32 \\
\hline Penza Oblast & -0.16 & -1.30 & -1.47 & -0.09 & -1.20 & -1.29 & -0.11 & -1.24 & -1.35 \\
\hline Perm Oblast & -0.11 & - & -0.11 & 0.15 & - & 0.15 & 0.07 & - & 0.07 \\
\hline Primorsky Krai & -0.14 & -2.81 & -2.95 & -0.11 & 1.13 & 1.01 & -0.12 & -0.20 & -0.33 \\
\hline Pskov Oblast & -0.22 & -1.42 & -1.65 & -0.14 & -0.30 & -0.43 & -0.17 & -0.67 & -0.84 \\
\hline Republic Adygeya & -0.22 & -2.42 & -2.63 & -0.03 & 0.58 & 0.55 & -0.09 & -0.43 & -0.52 \\
\hline Altay Republic & -0.11 & - & -0.11 & 0.08 & - & 0.08 & 0.02 & - & 0.02 \\
\hline $\begin{array}{l}\text { Republic Bashkor- } \\
\text { tostan }\end{array}$ & 0.09 & -2.92 & -2.84 & 0.26 & 0.08 & 0.34 & 0.20 & -0.93 & -0.73 \\
\hline Republic Buryatia & -0.12 & -2.67 & -2.79 & -0.07 & 0.30 & 0.23 & -0.09 & -0.70 & -0.79 \\
\hline Republic of Dagestan & 0.32 & 0.95 & 1.27 & 0.14 & -0.72 & -0.57 & 0.20 & -0.16 & 0.04 \\
\hline $\begin{array}{l}\text { Republic of Ingou- } \\
\text { shetia }\end{array}$ & -0.41 & - & -0.41 & -0.37 & -32.92 & -0.37 & -0.38 & - & -0.38 \\
\hline Republic of Kalmykia & -0.11 & -1.54 & -1.65 & 0.63 & -5.80 & -5.15 & 0.38 & -4.40 & -4.00 \\
\hline Republic Karelia & -0.19 & 0.01 & -0.18 & -0.01 & 1.26 & 1.25 & -0.07 & 0.84 & 0.77 \\
\hline Republic Komi & 0.02 & -1.00 & -0.98 & 0.22 & 0.04 & 0.26 & 0.15 & -0.31 & -0.16 \\
\hline Republic Mary-El & -0.18 & -1.76 & -1.95 & -0.14 & -3.35 & -3.49 & -0.15 & -2.82 & -2.98 \\
\hline Republic Mordovia & -0.16 & -1.48 & -1.65 & -0.01 & 0.72 & 0.71 & -0.06 & -0.02 & -0.08 \\
\hline $\begin{array}{l}\text { Republic of Sakha } \\
\text { (Yakutia) }\end{array}$ & 0.08 & -0.53 & -0.45 & 0.36 & -0.47 & -0.11 & 0.27 & -0.49 & -0.22 \\
\hline $\begin{array}{l}\text { Republic North } \\
\text { Ossetia (Alania) }\end{array}$ & -0.09 & - & -0.09 & 0.02 & - & 0.02 & -0.02 & - & -0.02 \\
\hline Republic Tatarstan & 0.08 & -2.55 & -2.47 & 0.41 & 1.47 & 1.88 & 0.30 & 0.11 & 0.41 \\
\hline
\end{tabular}




\begin{tabular}{|c|c|c|c|c|c|c|c|c|c|}
\hline 1 & 2 & 3 & 4 & 5 & 6 & 7 & 8 & 9 & 10 \\
\hline Republic of Tyva & -0.29 & - & -0.29 & -0.23 & - & -0.23 & -0.25 & - & -0.25 \\
\hline Republic Khakassia & -0.14 & -26.08 & -26.23 & -0.11 & 11.87 & 11.76 & -0.12 & -2.56 & -2.69 \\
\hline Rostov Oblast & -0.15 & -3.63 & -3.78 & 0.07 & 1.46 & 1.52 & -0.01 & -0.27 & -0.28 \\
\hline Ryazan Oblast & -0.25 & -0.64 & -0.89 & -0.02 & 0.08 & 0.06 & -0.10 & -0.16 & -0.26 \\
\hline Samara Oblast & 0.03 & -1.29 & -1.26 & 0.15 & 1.10 & 1.25 & 0.11 & 0.30 & 0.41 \\
\hline Saratov Oblast & -0.11 & -1.23 & -1.34 & -0.01 & -0.02 & -0.03 & -0.04 & -0.43 & -0.47 \\
\hline Sakhalin Oblast & -0.01 & -1.67 & -1.68 & 0.53 & -1.87 & -1.34 & 0.35 & -1.81 & -1.45 \\
\hline Sverdlovsk Oblast & 0.07 & -0.64 & -0.57 & 0.06 & 1.17 & 1.23 & 0.06 & 0.56 & 0.62 \\
\hline Smolensk Oblast & -0.34 & -3.81 & -4.15 & 0.04 & 1.03 & 1.07 & -0.09 & -0.61 & -0.70 \\
\hline Stavropol Krai & -0.20 & -1.67 & -1.87 & 0.04 & 0.65 & 0.70 & -0.04 & -0.13 & -0.17 \\
\hline Tambov Oblast & -0.28 & -0.67 & -0.94 & -0.22 & -0.94 & -1.17 & -0.24 & -0.85 & -1.09 \\
\hline Tver Oblast & -0.13 & -0.19 & -0.32 & 0.14 & -0.33 & -0.19 & 0.05 & -0.28 & -0.24 \\
\hline Tomsk Oblast & 0.00 & -1.28 & -1.28 & 0.13 & 1.03 & 1.16 & 0.08 & 0.25 & 0.34 \\
\hline Tula Oblast & -0.15 & 0.12 & -0.04 & -0.02 & 0.65 & 0.62 & -0.07 & 0.47 & 0.40 \\
\hline Tyumen Oblast & 0.35 & -0.22 & 0.13 & 0.75 & 2.77 & 3.51 & 0.61 & 1.76 & 2.37 \\
\hline Udmurtia Republic & -0.02 & -0.75 & -0.77 & 0.09 & 1.24 & 1.33 & 0.05 & 0.57 & 0.62 \\
\hline Ulyanovsk Oblast & -0.25 & -1.45 & -1.70 & -0.18 & -0.81 & -0.99 & -0.20 & -1.03 & -1.23 \\
\hline Khabarovsk Krai & -0.10 & - & -0.10 & 0.14 & - & 0.14 & 0.06 & - & 0.06 \\
\hline Chelyabinsk Oblast & -0.07 & -0.56 & -0.63 & 0.06 & -2.03 & -1.97 & 0.01 & -1.54 & -1.52 \\
\hline Chita Oblast & -0.14 & - & -0.14 & -0.03 & - & -0.03 & -0.07 & - & -0.07 \\
\hline Chuvash Republic & -0.15 & -0.13 & -0.28 & -0.05 & 1.23 & 1.18 & -0.09 & 0.77 & 0.69 \\
\hline $\begin{array}{l}\text { Chukotka autono- } \\
\text { mous okrug }\end{array}$ & -0.34 & - & -0.34 & -0.07 & -3.92 & -0.07 & -0.16 & - & -0.16 \\
\hline Yaroslavl Oblast & -0.22 & -0.46 & -0.68 & 0.08 & 2.01 & 2.09 & -0.02 & 1.18 & 1.16 \\
\hline
\end{tabular}

Table 2A-9

\section{Decomposition of Growth in GRP by the Regions for the Period between 1997 and 1998}

\begin{tabular}{lccccccc}
\hline & Far-Eastern & Volga & $\begin{array}{c}\text { North- } \\
\text { Western }\end{array}$ & Siberian & Ural & Central & Southern \\
\hline \multicolumn{1}{c}{1} & 2 & 3 & 4 & 5 & 6 & 7 & 8 \\
\hline GRP & -4.80 & -2.63 & -3.22 & -6.69 & -2.92 & 0.12 & -3.66 \\
I. Factor inputs & -3.54 & -2.41 & -1.75 & -4.00 & -1.34 & -0.85 & -3.95 \\
I.1 Labor (Employ- & -1.93 & -0.98 & -1.31 & -1.71 & -1.07 & -0.60 & -1.53 \\
ment) & & & & & & &
\end{tabular}




\begin{tabular}{lccccccc}
\hline \multicolumn{1}{c}{1} & 2 & 3 & 4 & 5 & 6 & 7 & 8 \\
\hline I.2 Capital & -1.61 & -1.43 & -0.44 & -2.29 & -0.27 & -0.25 & -2.42 \\
Fixed assets & -0.09 & 0.13 & -0.10 & -0.26 & 0.27 & 0.08 & 0.06 \\
$\quad$ Loading rate & -1.52 & -1.56 & -0.34 & -2.03 & -0.54 & -0.32 & -2.48 \\
Employment structure & -0.51 & -0.08 & 0.19 & -0.34 & 0.88 & 0.07 & -0.51 \\
II. Remainder & -0.75 & -0.14 & -1.65 & -2.36 & -2.46 & 0.90 & 0.80 \\
The Solow remainder & -2.78 & -1.76 & -1.81 & -4.73 & -2.12 & 0.65 & -2.18 \\
\hline
\end{tabular}

Table 2A-10

\section{Decomposition of Growth in GRP by the Regions for the Period between 1999 and 2002}

\begin{tabular}{|c|c|c|c|c|c|c|c|}
\hline & $\begin{array}{c}\text { Far- } \\
\text { Eastern }\end{array}$ & Volga & $\begin{array}{c}\text { North- } \\
\text { Western }\end{array}$ & Siberian & Ural & Central & Southern \\
\hline GRP & 4.60 & 5.52 & 7.46 & 5.60 & 6.22 & 7.72 & 8.17 \\
\hline I. Factor inputs & 0.91 & 1.72 & 1.80 & 1.80 & 2.37 & 2.43 & 2.25 \\
\hline $\begin{array}{l}\text { I.1 Labor (Employ- } \\
\text { ment) }\end{array}$ & 0.69 & 0.80 & 0.67 & 0.63 & 0.71 & 0.63 & 1.34 \\
\hline I.2 Capital & 0.22 & 0.92 & 1.13 & 1.16 & 1.66 & 1.80 & 0.90 \\
\hline Fixed assets & 0.05 & 0.28 & 0.17 & -0.19 & 0.53 & 0.35 & 0.40 \\
\hline Loading rate & 0.17 & 0.64 & 0.96 & 1.35 & 1.13 & 1.45 & 0.50 \\
\hline $\begin{array}{l}\text { Employment struc- } \\
\text { ture }\end{array}$ & -0.33 & -0.30 & -0.04 & -0.45 & -0.29 & -0.43 & -0.52 \\
\hline II. Remainder & 4.02 & 4.11 & 5.69 & 4.25 & 4.14 & 5.72 & 6.44 \\
\hline The Solow remainder & 3.86 & 4.43 & 6.62 & 5.15 & 4.96 & 6.73 & 6.41 \\
\hline
\end{tabular}

Table 2A-11

Decomposition of the Regions' Output Growth Rates in 1997-2002

\begin{tabular}{|c|c|c|c|c|c|c|c|c|}
\hline & \multirow[b]{2}{*}{ GRP } & \multirow{2}{*}{$\begin{array}{l}\text { I. Factor } \\
\text { inputs }\end{array}$} & \multirow{2}{*}{$\begin{array}{l}\text { I.1 Labor } \\
\text { (Em- } \\
\text { ployment) }\end{array}$} & \multirow{2}{*}{$\begin{array}{l}\text { I.2 Capi- } \\
\text { tal }\end{array}$} & \multicolumn{2}{|c|}{ of which } & \multirow{2}{*}{$\begin{array}{c}\text { Employment } \\
\text { structure }\end{array}$} & \multirow{2}{*}{$\begin{array}{l}\text { II. Remain } \\
\text { der }\end{array}$} \\
\hline & & & & & $\begin{array}{l}\text { Fixed } \\
\text { assets }\end{array}$ & $\begin{array}{c}\begin{array}{c}\text { Loading } \\
\text { rate }\end{array} \\
\end{array}$ & & \\
\hline 1 & 2 & 3 & 4 & 5 & 6 & 7 & 8 & 9 \\
\hline Altay Krai & 0.62 & -1.57 & 0.03 & -1.60 & -0.17 & -1.42 & -0.43 & 2.71 \\
\hline Amour Oblast & -0.08 & -0.98 & -0.48 & -0.50 & -0.09 & -0.42 & -0.69 & 1.74 \\
\hline Arkhangel' Oblast & 4.00 & -0.14 & -0.27 & 0.13 & -0.05 & 0.19 & -0.25 & 4.44 \\
\hline Astrakhan Oblast & 6.96 & 0.53 & -0.07 & 0.59 & 0.26 & 0.33 & -0.96 & 7.42 \\
\hline Belgorod Oblast & 4.98 & 1.28 & 0.27 & 1.02 & 0.10 & 0.92 & -0.37 & 4.08 \\
\hline Bryansk Oblast & 2.34 & 0.19 & 0.31 & -0.12 & -0.24 & 0.12 & -0.79 & 2.91 \\
\hline Vladimir Oblast & 3.11 & 0.81 & 0.29 & 0.52 & -0.07 & 0.59 & -0.23 & 2.57 \\
\hline
\end{tabular}




\begin{tabular}{|c|c|c|c|c|c|c|c|c|}
\hline 1 & 2 & 3 & 4 & 5 & 6 & 7 & 8 & 9 \\
\hline Volgograd Oblast & 2.33 & -2.46 & -0.18 & -2.28 & -0.10 & -2.18 & -0.82 & 5.62 \\
\hline Vologda Oblast & 3.10 & 0.60 & 0.24 & 0.36 & 0.00 & 0.35 & -0.01 & 2.54 \\
\hline Voronezh Oblast & 2.17 & -0.19 & 0.38 & -0.57 & -0.17 & -0.40 & -0.48 & 2.85 \\
\hline $\begin{array}{l}\text { the city of Mos- } \\
\text { cow }\end{array}$ & 6.42 & 3.68 & 0.42 & 3.26 & 1.07 & 2.19 & -0.12 & 2.81 \\
\hline $\begin{array}{l}\text { the city of Saint- } \\
\text { Petersburg }\end{array}$ & 4.73 & 1.71 & 0.20 & 1.50 & 0.19 & 1.32 & 0.24 & 2.82 \\
\hline $\begin{array}{l}\text { Jewish Autono- } \\
\text { mous Oblast }\end{array}$ & -1.20 & -0.31 & -0.16 & -0.15 & -0.12 & -0.03 & -0.95 & 0.19 \\
\hline Ivanovo Oblast & -0.10 & -0.61 & -0.78 & 0.17 & -0.19 & 0.35 & -0.25 & 0.89 \\
\hline Irkutsk Oblast & -2.08 & 0.33 & 0.07 & 0.26 & -0.19 & 0.45 & -0.60 & -1.74 \\
\hline $\begin{array}{l}\text { Kabardino-Balkar } \\
\text { Republic }\end{array}$ & 6.89 & -0.25 & 1.17 & -1.42 & 0.09 & -1.51 & -0.47 & 7.56 \\
\hline Kaliningrad Oblast & 2.76 & 0.58 & 0.32 & 0.26 & -0.06 & 0.31 & -0.42 & 2.64 \\
\hline Kaluga Oblast & 1.07 & -0.08 & -0.52 & 0.44 & -0.02 & 0.46 & -0.29 & 1.44 \\
\hline Kamchatka Oblast & -3.40 & -2.11 & -0.82 & -1.29 & -0.17 & -1.12 & -0.30 & -0.94 \\
\hline $\begin{array}{l}\text { Karachay- } \\
\text { Cherkessya Re- } \\
\text { public }\end{array}$ & 4.17 & 0.46 & 0.52 & -0.07 & -0.12 & 0.06 & -0.70 & 4.27 \\
\hline Kemerovo Oblast & 1.57 & -0.61 & -0.76 & 0.15 & 0.07 & 0.08 & -0.26 & 2.49 \\
\hline Kirov Oblast & 0.00 & 0.29 & 0.31 & -0.03 & -0.19 & 0.16 & -0.39 & 0.11 \\
\hline Kostroma Oblast & 1.84 & -0.77 & -0.46 & -0.31 & 0.00 & -0.31 & -0.16 & 2.77 \\
\hline KrasnodarKrai & 3.07 & 1.76 & 0.59 & 1.16 & 0.49 & 0.67 & -0.24 & 1.68 \\
\hline Krasnoyarsk Krai & 2.16 & 0.01 & 0.15 & -0.14 & -0.02 & -0.12 & -0.50 & 2.68 \\
\hline Kurgan Oblast & 1.19 & -1.23 & -0.38 & -0.85 & -0.17 & -0.68 & -0.62 & 2.95 \\
\hline Kursk Oblast & 3.41 & 0.44 & 0.65 & -0.20 & -0.19 & -0.01 & -0.58 & 3.51 \\
\hline Leningrad Oblast & 6.12 & 0.47 & 0.16 & 0.31 & 0.31 & - & 0.20 & 5.50 \\
\hline Lipetsk Oblast & 2.23 & -0.86 & 0.37 & -1.23 & -0.12 & -1.11 & -0.28 & 3.35 \\
\hline Magadan Oblast & -3.37 & -3.51 & -2.26 & -1.25 & -0.07 & -1.18 & -0.21 & -0.06 \\
\hline Moscow Oblast & 4.73 & 0.82 & 0.47 & 0.34 & 0.34 & - & -0.13 & 4.05 \\
\hline Murmansk Oblast & 1.64 & -0.82 & -0.47 & -0.36 & -0.12 & -0.24 & -0.51 & 2.96 \\
\hline $\begin{array}{l}\text { Nizhny Novgorod } \\
\text { Oblast }\end{array}$ & 3.89 & -0.45 & -0.15 & -0.30 & 0.00 & -0.29 & 0.15 & 4.04 \\
\hline Novgorod Oblast & 3.46 & -1.24 & -0.79 & -0.44 & 0.08 & -0.52 & 0.26 & 4.43 \\
\hline $\begin{array}{l}\text { Novosibirsk } \\
\text { Oblast }\end{array}$ & 3.52 & -1.03 & -0.16 & -0.87 & -0.11 & -0.76 & -0.31 & 4.94 \\
\hline Omsk Oblast & 1.39 & -1.07 & 0.18 & -1.25 & -0.15 & -1.10 & 0.21 & 2.36 \\
\hline Orenburg Oblast & 2.08 & 0.97 & 0.61 & 0.36 & 0.01 & 0.35 & -0.39 & 1.56 \\
\hline Orel Oblast & 5.97 & 0.24 & 0.56 & -0.32 & -0.06 & -0.27 & -0.39 & 6.08 \\
\hline
\end{tabular}




\begin{tabular}{|c|c|c|c|c|c|c|c|c|}
\hline 1 & 2 & 3 & 4 & 5 & 6 & 7 & 8 & 9 \\
\hline Penza Oblast & 1.92 & -1.17 & 0.18 & -1.35 & -0.11 & -1.24 & -0.55 & 3.67 \\
\hline Perm Oblast & 3.62 & 0.38 & 0.32 & 0.07 & 0.07 & - & -0.04 & 3.32 \\
\hline Primorsky Krai & 0.31 & -0.06 & 0.27 & -0.33 & -0.12 & -0.20 & -0.38 & 0.73 \\
\hline Pskov Oblast & 0.82 & -0.52 & 0.32 & -0.84 & -0.17 & -0.67 & -0.50 & 1.95 \\
\hline Republic Adygeya & -0.20 & -0.98 & -0.46 & -0.52 & -0.09 & -0.43 & -0.82 & 1.68 \\
\hline Altay Republic & 1.71 & -0.07 & -0.08 & 0.02 & 0.02 & - & -0.79 & 2.60 \\
\hline $\begin{array}{l}\text { Republic Bashkor- } \\
\text { tostan }\end{array}$ & 1.84 & -0.60 & 0.13 & -0.73 & 0.20 & -0.93 & -0.28 & 2.74 \\
\hline Republic Buryatia & 3.37 & -1.94 & -1.16 & -0.79 & -0.09 & -0.70 & -0.81 & 6.10 \\
\hline $\begin{array}{l}\text { Republic of } \\
\text { Dagestan }\end{array}$ & 5.65 & 1.81 & 1.77 & 0.04 & 0.20 & -0.16 & -1.24 & 4.41 \\
\hline $\begin{array}{l}\text { Republic of In- } \\
\text { goushetia }\end{array}$ & -2.63 & 1.30 & 1.68 & -0.38 & -0.38 & - & -1.57 & -1.86 \\
\hline $\begin{array}{l}\text { Republic of Kal- } \\
\text { mykia }\end{array}$ & 4.15 & -6.35 & -2.35 & -4.00 & 0.38 & -4.40 & -0.83 & 11.40 \\
\hline Republic Karelia & 2.24 & -0.11 & -0.89 & 0.77 & -0.07 & 0.84 & -0.28 & 2.76 \\
\hline Republic Komi & 1.16 & -0.42 & -0.27 & -0.16 & 0.15 & -0.31 & -0.40 & 2.03 \\
\hline Republic Mary-El & -0.41 & -3.02 & -0.04 & -2.98 & -0.15 & -2.82 & -0.34 & 2.73 \\
\hline $\begin{array}{l}\text { Republic Mor- } \\
\text { dovia }\end{array}$ & 4.28 & 0.01 & 0.09 & -0.08 & -0.06 & -0.02 & -0.12 & 4.43 \\
\hline $\begin{array}{l}\text { Republic of Sakha } \\
\text { (Yakutia) }\end{array}$ & 1.02 & -1.46 & -1.24 & -0.22 & 0.27 & -0.49 & -0.38 & 2.87 \\
\hline $\begin{array}{l}\text { Republic North } \\
\text { Ossetia (Alania) }\end{array}$ & 6.63 & 1.27 & 1.29 & -0.02 & -0.02 & - & -0.79 & 6.21 \\
\hline Republic Tatarstan & 3.20 & 0.60 & 0.19 & 0.41 & 0.30 & 0.11 & -0.12 & 2.80 \\
\hline Republic of Tyva & 3.41 & -2.04 & -1.79 & -0.25 & -0.25 & - & -0.30 & 5.79 \\
\hline $\begin{array}{l}\text { Republic Khakas- } \\
\text { sia }\end{array}$ & -0.42 & -2.36 & 0.33 & -2.69 & -0.12 & -2.56 & -0.67 & -2.23 \\
\hline Rostov Oblast & 5.93 & -0.16 & 0.12 & -0.28 & -0.01 & -0.27 & -0.62 & 6.78 \\
\hline Ryazan Oblast & 2.42 & -0.33 & -0.07 & -0.26 & -0.10 & -0.16 & -0.30 & 3.03 \\
\hline Samara Oblast & 2.60 & 0.73 & 0.32 & 0.41 & 0.11 & 0.30 & -0.26 & 2.18 \\
\hline Saratov Oblast & 3.82 & -0.44 & 0.03 & -0.47 & -0.04 & -0.43 & -0.54 & 4.80 \\
\hline Sakhalin Oblast & 2.20 & -1.78 & -0.33 & -1.45 & 0.35 & -1.81 & -0.44 & 4.38 \\
\hline Sverdlovsk Oblast & 2.16 & 0.75 & 0.13 & 0.62 & 0.06 & 0.56 & -0.18 & 1.66 \\
\hline Smolensk Oblast & 3.69 & -0.55 & 0.15 & -0.70 & -0.09 & -0.61 & -0.29 & 4.68 \\
\hline Stavropol Krai & 2.61 & -0.09 & 0.08 & -0.17 & -0.04 & -0.13 & -0.41 & 3.09 \\
\hline Tambov Oblast & 5.33 & -1.45 & -0.36 & -1.09 & -0.24 & -0.85 & -0.55 & 7.25 \\
\hline Tver Oblast & 1.39 & -0.45 & -0.21 & -0.24 & 0.05 & -0.28 & -0.30 & 2.09 \\
\hline Tomsk Oblast & 2.96 & 0.12 & -0.22 & 0.34 & 0.08 & 0.25 & -0.04 & 2.99 \\
\hline Tula Oblast & 0.70 & -0.27 & -0.68 & 0.40 & -0.07 & 0.47 & -0.35 & 1.36 \\
\hline
\end{tabular}




\begin{tabular}{|c|c|c|c|c|c|c|c|c|}
\hline 1 & 2 & 3 & 4 & 5 & 6 & 7 & 8 & 9 \\
\hline Tyumen Oblast & 3.89 & 2.50 & 0.12 & 2.37 & 0.61 & 1.76 & 0.12 & 1.27 \\
\hline $\begin{array}{l}\text { Udmurtia Repub- } \\
\text { lic }\end{array}$ & 2.21 & 1.12 & 0.50 & 0.62 & 0.05 & 0.57 & -0.26 & 1.42 \\
\hline Ulyanovsk Oblast & 1.65 & -1.48 & -0.25 & -1.23 & -0.20 & -1.03 & -0.32 & 3.51 \\
\hline Khabarovsk Krai & 5.13 & 1.03 & 0.97 & 0.06 & 0.06 & - & -0.49 & 4.65 \\
\hline $\begin{array}{l}\text { Chelyabinsk } \\
\text { Oblast }\end{array}$ & 0.76 & -1.29 & 0.24 & -1.52 & 0.01 & -1.54 & -0.24 & 2.32 \\
\hline Chita Oblast & 0.40 & -0.38 & -0.31 & -0.07 & -0.07 & - & -1.02 & 1.95 \\
\hline Chuvash Republic & -0.02 & 1.22 & 0.53 & 0.69 & -0.09 & 0.77 & -0.45 & -0.74 \\
\hline $\begin{array}{l}\text { Chukotka autono- } \\
\text { mous okrug }\end{array}$ & 1.55 & -3.45 & -3.29 & -0.16 & -0.16 & - & -0.02 & 5.65 \\
\hline Yaroslavl Oblast & 4.44 & 1.36 & 0.20 & 1.16 & -0.02 & 1.18 & -0.22 & 3.40 \\
\hline
\end{tabular}

Table 2A-12

\section{Results of Assessment of Output Growth Rates and Contribution of Labor Input by the Regions}

\begin{tabular}{|c|c|c|c|c|c|c|c|c|c|}
\hline Region & $\begin{array}{c}\text { Number } \\
\text { of the } \\
\text { em- } \\
\text { ployed }\end{array}$ & $\begin{array}{c}\text { Average } \\
\text { worked } \\
\text { time }\end{array}$ & $\begin{array}{c}\text { Gender } \\
\text { struc- } \\
\text { ture }\end{array}$ & $\begin{array}{l}\text { Labor } \\
\text { input }\end{array}$ & Region & $\begin{array}{c}\text { Number } \\
\text { of the } \\
\text { em- } \\
\text { ployed }\end{array}$ & $\begin{array}{c}\text { Average } \\
\text { worked } \\
\text { time }\end{array}$ & $\begin{array}{l}\text { Gender } \\
\text { structure }\end{array}$ & $\begin{array}{c}\text { Labor } \\
\text { input }\end{array}$ \\
\hline 1 & 2 & 3 & 4 & 5 & 6 & 7 & 8 & 9 & 10 \\
\hline $\begin{array}{l}\text { Russian Federa- } \\
\text { tion }\end{array}$ & -0.10 & 0.03 & 0.60 & 0.53 & Perm Oblast & 0.32 & -0.01 & -0.07 & 0.24 \\
\hline Altay Krai & 0.03 & 0.39 & 0.37 & 0.79 & Primorsky Krai & 0.27 & -1.26 & -1.11 & -2.11 \\
\hline Amour Oblast & -0.48 & 1.46 & -0.36 & 0.62 & Pskov Oblast & 0.32 & 1.04 & 0.71 & 2.07 \\
\hline Arkhangel' Oblast & -0.27 & 0.65 & 0.28 & 0.66 & Republic Adygeya & -0.46 & -0.69 & 0.33 & -0.81 \\
\hline Astrakhan Oblast & -0.07 & 0.23 & 0.34 & 0.50 & Altay Republic & -0.08 & -0.16 & -1.09 & -1.34 \\
\hline Belgorod Oblast & 0.27 & -0.30 & 0.77 & 0.73 & $\begin{array}{l}\text { Republic Bashkor- } \\
\text { tostan }\end{array}$ & 0.13 & -0.24 & 0.44 & 0.33 \\
\hline Bryansk Oblast & 0.31 & 1.09 & 0.62 & 2.01 & Republic Buryatia & -1.16 & -0.06 & -1.18 & -2.40 \\
\hline Vladimir Oblast & 0.29 & 0.28 & 0.27 & 0.84 & $\begin{array}{l}\text { Republic of } \\
\text { Dagestan }\end{array}$ & 1.77 & -0.02 & -1.61 & 0.14 \\
\hline Volgograd Oblast & -0.18 & -0.52 & 0.42 & -0.28 & $\begin{array}{l}\text { Republic of In- } \\
\text { goushetia }\end{array}$ & 1.68 & -0.54 & -1.57 & -0.43 \\
\hline Vologda Oblast & 0.24 & 0.27 & 9.58 & 10.10 & $\begin{array}{l}\text { Republic of Kal- } \\
\text { mykia }\end{array}$ & -2.35 & 0.80 & -1.11 & -2.66 \\
\hline Voronezh Oblast & 0.38 & -0.72 & 1.02 & 0.68 & Republic Karelia & -0.89 & 0.03 & 0.39 & -0.47 \\
\hline $\begin{array}{l}\text { the city of Mos- } \\
\text { cow }\end{array}$ & 0.42 & -0.06 & 1.82 & 2.18 & Republic Komi & -0.27 & 0.64 & -0.43 & -0.05 \\
\hline $\begin{array}{l}\text { the city of Saint- } \\
\text { Petersburg }\end{array}$ & 0.20 & 0.35 & -0.81 & -0.26 & Republic Mary-El & -0.04 & -0.09 & 0.12 & -0.02 \\
\hline
\end{tabular}




\begin{tabular}{|c|c|c|c|c|c|c|c|c|c|}
\hline 1 & 2 & 3 & 4 & 5 & 6 & 7 & 8 & 9 & 10 \\
\hline $\begin{array}{l}\text { Jewish Autono- } \\
\text { mous Oblast }\end{array}$ & -0.16 & -0.21 & -0.22 & -0.59 & $\begin{array}{l}\text { Republic Mor- } \\
\text { dovia }\end{array}$ & 0.09 & -0.30 & 0.44 & 0.23 \\
\hline Ivanovo Oblast & -0.78 & 0.53 & 0.07 & -0.18 & $\begin{array}{l}\text { Republic of Sakha } \\
\text { (Yakutia) }\end{array}$ & -1.24 & -0.85 & -0.20 & -2.29 \\
\hline Irkutsk Oblast & 0.07 & 0.11 & -0.18 & 0.00 & $\begin{array}{l}\text { Republic North } \\
\text { Ossetia (Alania) }\end{array}$ & 1.29 & -0.74 & -0.12 & 0.43 \\
\hline $\begin{array}{l}\text { Kabardino-Balkar } \\
\text { Republic }\end{array}$ & 1.17 & -0.71 & -0.12 & 0.34 & Republic Tatarstan & 0.19 & 0.13 & 0.15 & 0.47 \\
\hline Kaliningrad Oblast & 0.32 & -0.46 & -0.35 & -0.49 & Republic of Tyva & -1.79 & 0.45 & -0.06 & -1.40 \\
\hline Kaluga Oblast & -0.52 & -0.63 & 0.16 & -0.99 & $\begin{array}{l}\text { Republic Khakas- } \\
\text { sia }\end{array}$ & 0.33 & -0.92 & 0.46 & -0.13 \\
\hline Kamchatka Oblast & -0.82 & -0.14 & -1.05 & -2.02 & Rostov Oblast & 0.12 & 0.51 & 0.19 & 0.82 \\
\hline $\begin{array}{l}\text { Karachay- } \\
\text { Cherkessya Re- } \\
\text { public }\end{array}$ & 0.52 & -1.03 & -0.32 & -0.83 & Ryazan Oblast & -0.07 & -0.23 & 1.37 & 1.07 \\
\hline Kemerovo Oblast & -0.76 & 0.42 & 0.33 & -0.01 & Samara Oblast & 0.32 & 0.46 & 0.00 & 0.79 \\
\hline Kirov Oblast & 0.31 & -0.10 & 0.40 & 0.62 & Saratov Oblast & 0.03 & 0.23 & 0.43 & 0.69 \\
\hline Kostroma Oblast & -0.46 & -0.46 & 0.39 & -0.53 & Sakhalin Oblast & -0.33 & 0.89 & 0.72 & 1.28 \\
\hline KrasnodarKrai & 0.59 & -0.97 & 0.16 & -0.21 & Sverdlovsk Oblast & 0.13 & 0.40 & 0.29 & 0.82 \\
\hline Krasnoyarsk Krai & 0.15 & 0.01 & -0.24 & -0.08 & Smolensk Oblast & 0.15 & -0.10 & 0.25 & 0.29 \\
\hline Kurgan Oblast & -0.38 & 0.24 & 0.61 & 0.47 & Stavropol Krai & 0.08 & 0.56 & -0.02 & 0.61 \\
\hline Kursk Oblast & 0.65 & -1.37 & -0.06 & -0.79 & Tambov Oblast & -0.36 & -0.57 & 0.43 & -0.50 \\
\hline Leningrad Oblast & 0.16 & 0.23 & -0.30 & 0.09 & Tver Oblast & -0.21 & -0.03 & 0.22 & -0.02 \\
\hline Lipetsk Oblast & 0.37 & -0.34 & 0.58 & 0.62 & Tomsk Oblast & -0.22 & 0.85 & 0.42 & 1.05 \\
\hline Magadan Oblast & -2.26 & -0.80 & 2.30 & -0.76 & Tula Oblast & -0.68 & 0.38 & -0.01 & -0.30 \\
\hline Moscow Oblast & 0.47 & 0.07 & 1.16 & 1.70 & Tyumen Oblast & 0.12 & 0.07 & 0.55 & 0.75 \\
\hline Murmansk Oblast & -0.47 & -1.44 & 0.59 & -1.31 & $\begin{array}{l}\text { Udmurtia Repub- } \\
\text { lic }\end{array}$ & 0.50 & -0.02 & -0.06 & 0.43 \\
\hline $\begin{array}{l}\text { Nizhny Novgorod } \\
\text { Oblast }\end{array}$ & -0.15 & 0.23 & 1.09 & 1.17 & Ulyanovsk Oblast & -0.25 & 0.02 & 0.32 & 0.09 \\
\hline Novgorod Oblast & -0.79 & 0.16 & 0.33 & -0.30 & Khabarovsk Krai & 0.97 & 0.03 & 0.61 & 1.61 \\
\hline $\begin{array}{l}\text { Novosibirsk } \\
\text { Oblast }\end{array}$ & -0.16 & 0.05 & 0.23 & 0.12 & $\begin{array}{l}\text { Chelyabinsk } \\
\text { Oblast }\end{array}$ & 0.24 & 0.60 & 0.02 & 0.85 \\
\hline Omsk Oblast & 0.18 & -0.20 & -0.14 & -0.16 & Chita Oblast & -0.31 & -0.79 & -0.19 & -1.29 \\
\hline Orenburg Oblast & 0.61 & -0.12 & 0.46 & 0.94 & Chuvash Republic & 0.53 & 0.03 & 0.30 & 0.86 \\
\hline Orel Oblast & 0.56 & 0.27 & 0.21 & 1.04 & $\begin{array}{l}\text { Chukotka autono- } \\
\text { mous okrug }\end{array}$ & -3.29 & 1.06 & -1.54 & -3.77 \\
\hline Penza Oblast & 0.18 & -0.64 & 0.56 & 0.10 & Yaroslavl Oblast & 0.20 & 0.32 & 0.14 & 0.67 \\
\hline
\end{tabular}


Table 2A-13

Decomposition of the GRP Growth by the Regions for the period 2000-2002

\begin{tabular}{|c|c|c|c|c|c|c|c|c|c|c|}
\hline & \multirow[b]{2}{*}{ GRP } & \multirow[b]{2}{*}{$\begin{array}{l}\text { I. Factor } \\
\text { inputs }\end{array}$} & \multirow[b]{2}{*}{$\begin{array}{c}\text { I.1 } \\
\text { Labor }\end{array}$} & \multicolumn{3}{|c|}{ of which } & \multirow[b]{2}{*}{$\begin{array}{c}\text { I.2 } \\
\text { Capital }\end{array}$} & \multicolumn{2}{|c|}{ of which } & \multirow{2}{*}{$\begin{array}{c}\text { II. } \\
\text { Re- } \\
\text { mained }\end{array}$} \\
\hline & & & & $\begin{array}{c}\text { Employ- } \\
\text { ment }\end{array}$ & $\begin{array}{c}\text { Worked } \\
\text { time }\end{array}$ & $\begin{array}{l}\text { Struc- } \\
\text { ture }\end{array}$ & & $\begin{array}{l}\text { Fixed } \\
\text { assets }\end{array}$ & $\begin{array}{l}\text { Loading } \\
\text { rate }\end{array}$ & \\
\hline 1 & 2 & 3 & 4 & 5 & 6 & 7 & 8 & 9 & 10 & 11 \\
\hline $\begin{array}{l}\text { Russian Federa- } \\
\text { tion }\end{array}$ & 7.13 & 2.11 & 1.00 & 0.36 & 0.03 & 0.60 & 1.11 & 0.23 & 0.88 & 5.02 \\
\hline Altay Krai & 7.18 & -0.53 & 0.54 & -0.22 & 0.39 & 0.37 & -1.07 & -0.15 & -0.92 & 7.71 \\
\hline Amour Oblast & 5.98 & 1.10 & 1.61 & 0.52 & 1.46 & -0.36 & -0.52 & 0.00 & -0.51 & 4.88 \\
\hline Arkhangel' Oblast & 7.32 & 2.27 & 1.89 & 0.96 & 0.65 & 0.28 & 0.38 & 0.09 & 0.29 & 5.05 \\
\hline Astrakhan Oblast & 8.64 & 2.21 & 0.93 & 0.36 & 0.23 & 0.34 & 1.28 & 0.53 & 0.75 & 6.43 \\
\hline Belgorod Oblast & 6.20 & 1.42 & 0.39 & -0.08 & -0.30 & 0.77 & 1.03 & 0.11 & 0.92 & 4.78 \\
\hline Bryansk Oblast & 7.90 & 3.21 & 2.15 & 0.44 & 1.09 & 0.62 & 1.06 & -0.18 & 1.23 & 4.70 \\
\hline Vladimir Oblast & 5.67 & 1.46 & 0.48 & -0.07 & 0.28 & 0.27 & 0.98 & -0.03 & 1.01 & 4.21 \\
\hline Volgograd Oblast & 8.16 & -0.48 & 0.84 & 0.94 & -0.52 & 0.42 & -1.32 & -0.05 & -1.28 & 8.64 \\
\hline Vologda Oblast & 3.83 & 11.33 & 10.77 & 0.92 & 0.27 & 9.58 & 0.55 & 0.02 & 0.54 & -7.49 \\
\hline Voronezh Oblast & 3.36 & -0.49 & 0.07 & -0.23 & -0.72 & 1.02 & -0.56 & -0.11 & -0.45 & 3.85 \\
\hline $\begin{array}{l}\text { the city of Mos- } \\
\text { cow }\end{array}$ & 9.84 & 6.61 & 1.92 & 0.16 & -0.06 & 1.82 & 4.68 & 1.26 & 3.42 & 3.23 \\
\hline $\begin{array}{l}\text { the city of Saint- } \\
\text { Petersburg }\end{array}$ & 10.12 & 2.01 & -0.23 & 0.23 & 0.35 & -0.81 & 2.24 & 0.31 & 1.93 & 8.11 \\
\hline $\begin{array}{l}\text { Jewish Autono- } \\
\text { mous Oblast }\end{array}$ & 6.57 & 0.88 & 1.04 & 1.48 & -0.21 & -0.22 & -0.17 & -0.13 & -0.04 & 5.69 \\
\hline Ivanovo Oblast & 4.94 & 0.91 & 0.22 & -0.38 & 0.53 & 0.07 & 0.69 & -0.17 & 0.86 & 4.03 \\
\hline Irkutsk Oblast & 3.06 & 0.76 & 0.21 & 0.28 & 0.11 & -0.18 & 0.55 & -0.16 & 0.71 & 2.30 \\
\hline $\begin{array}{l}\text { Kabardino-Balkar } \\
\text { Republic }\end{array}$ & 11.88 & -2.37 & -0.01 & 0.83 & -0.71 & -0.12 & -2.37 & 0.14 & -2.51 & 14.25 \\
\hline Kaliningrad Oblast & 8.77 & 0.64 & -0.34 & 0.48 & -0.46 & -0.35 & 0.98 & 0.03 & 0.95 & 8.12 \\
\hline Kaluga Oblast & 5.83 & -0.51 & -0.96 & -0.49 & -0.63 & 0.16 & 0.45 & 0.05 & 0.40 & 6.34 \\
\hline Kamchatka Oblast & -0.69 & -1.00 & -0.69 & 0.50 & -0.14 & -1.05 & -0.31 & -0.09 & -0.21 & 0.31 \\
\hline $\begin{array}{l}\text { Karachay- } \\
\text { Cherkessya Re- } \\
\text { public }\end{array}$ & 10.39 & -0.47 & 0.08 & 1.43 & -1.03 & -0.32 & -0.55 & -0.10 & -0.44 & 10.86 \\
\hline Kemerovo Oblast & 4.93 & 1.50 & 0.98 & 0.24 & 0.42 & 0.33 & 0.52 & 0.04 & 0.48 & 3.42 \\
\hline Kirov Oblast & 1.75 & -0.46 & -0.29 & -0.60 & -0.10 & 0.40 & -0.16 & -0.15 & -0.01 & 2.21 \\
\hline Kostroma Oblast & 3.30 & -1.69 & -0.48 & -0.40 & -0.46 & 0.39 & -1.21 & 0.08 & -1.29 & 4.99 \\
\hline KrasnodarKrai & 5.34 & 1.58 & -0.06 & 0.75 & -0.97 & 0.16 & 1.64 & 0.82 & 0.82 & 3.76 \\
\hline Krasnoyarsk Krai & 5.00 & 0.12 & 0.22 & 0.44 & 0.01 & -0.24 & -0.10 & 0.04 & -0.14 & 4.88 \\
\hline
\end{tabular}




\begin{tabular}{|c|c|c|c|c|c|c|c|c|c|c|}
\hline 1 & 2 & 3 & 4 & 5 & 6 & 7 & 8 & 9 & 10 & 11 \\
\hline Kurgan Oblast & 3.15 & -0.14 & 0.38 & -0.47 & 0.24 & 0.61 & -0.52 & -0.15 & -0.37 & 3.29 \\
\hline Kursk Oblast & 5.27 & -2.74 & -1.40 & 0.04 & -1.37 & -0.06 & -1.34 & -0.14 & -1.20 & 8.01 \\
\hline Leningrad Oblast & 11.73 & 0.76 & 0.19 & 0.26 & 0.23 & -0.30 & 0.57 & 0.57 & - & 10.97 \\
\hline Lipetsk Oblast & 7.06 & -0.31 & 0.28 & 0.03 & -0.34 & 0.58 & -0.59 & -0.07 & -0.51 & 7.37 \\
\hline Magadan Oblast & 3.10 & -1.35 & 0.77 & -0.73 & -0.80 & 2.30 & -2.13 & -0.09 & -2.04 & 4.45 \\
\hline Moscow Oblast & 6.07 & 2.36 & 1.96 & 0.73 & 0.07 & 1.16 & 0.40 & 0.40 & - & 3.71 \\
\hline Murmansk Oblast & 1.20 & -0.35 & -0.16 & 0.69 & -1.44 & 0.59 & -0.19 & -0.06 & -0.13 & 1.54 \\
\hline $\begin{array}{l}\text { Nizhny Novgorod } \\
\text { Oblast }\end{array}$ & 7.66 & 0.95 & 1.52 & 0.19 & 0.23 & 1.09 & -0.56 & -0.01 & -0.55 & 6.71 \\
\hline Novgorod Oblast & 4.04 & 0.00 & 0.62 & 0.13 & 0.16 & 0.33 & -0.62 & 0.20 & -0.82 & 4.04 \\
\hline $\begin{array}{l}\text { Novosibirsk } \\
\text { Oblast }\end{array}$ & 9.58 & 0.35 & 0.47 & 0.19 & 0.05 & 0.23 & -0.13 & -0.07 & -0.05 & 9.23 \\
\hline Omsk Oblast & 9.04 & 0.22 & -0.31 & 0.03 & -0.20 & -0.14 & 0.53 & -0.13 & 0.66 & 8.82 \\
\hline Orenburg Oblast & 4.99 & 1.94 & 0.95 & 0.61 & -0.12 & 0.46 & 0.99 & 0.06 & 0.93 & 3.05 \\
\hline Orel Oblast & 8.69 & -0.29 & 0.57 & 0.09 & 0.27 & 0.21 & -0.86 & 0.03 & -0.89 & 8.97 \\
\hline Penza Oblast & 4.24 & -1.65 & 0.12 & 0.20 & -0.64 & 0.56 & -1.77 & -0.08 & -1.69 & 5.89 \\
\hline Perm Oblast & 4.80 & 1.33 & 1.12 & 1.21 & -0.01 & -0.07 & 0.21 & 0.21 & - & 3.47 \\
\hline Primorsky Krai & 0.86 & -1.19 & -1.48 & 0.89 & -1.26 & -1.11 & 0.29 & -0.10 & 0.39 & 2.05 \\
\hline Pskov Oblast & 3.61 & 0.75 & 1.45 & -0.30 & 1.04 & 0.71 & -0.70 & -0.13 & -0.57 & 2.85 \\
\hline Republic Adygeya & 2.48 & -0.94 & -0.41 & -0.06 & -0.69 & 0.33 & -0.53 & -0.02 & -0.51 & 3.42 \\
\hline Altay Republic & 7.29 & -0.86 & -1.01 & 0.25 & -0.16 & -1.09 & 0.15 & 0.15 & - & 8.15 \\
\hline $\begin{array}{l}\text { Republic Bashkor- } \\
\text { tostan }\end{array}$ & 5.28 & 0.41 & 0.41 & 0.21 & -0.24 & 0.44 & 0.01 & 0.30 & -0.30 & 4.87 \\
\hline Republic Buryatia & 5.70 & -1.66 & -1.30 & -0.07 & -0.06 & -1.18 & -0.35 & -0.06 & -0.29 & 7.35 \\
\hline $\begin{array}{l}\text { Republic of } \\
\text { Dagestan }\end{array}$ & 13.93 & -1.75 & -1.11 & 0.52 & -0.02 & -1.61 & -0.64 & 0.17 & -0.81 & 15.68 \\
\hline $\begin{array}{l}\text { Republic of In- } \\
\text { goushetia }\end{array}$ & 3.33 & 1.08 & 1.44 & 3.55 & -0.54 & -1.57 & -0.36 & -0.36 & -41.65 & 2.25 \\
\hline $\begin{array}{l}\text { Republic of Kal- } \\
\text { mykia }\end{array}$ & 13.38 & -6.49 & -0.47 & -0.16 & 0.80 & -1.11 & -6.02 & 0.87 & -6.89 & 19.88 \\
\hline Republic Karelia & 5.96 & 1.98 & 1.12 & 0.70 & 0.03 & 0.39 & 0.86 & 0.03 & 0.83 & 3.98 \\
\hline Republic Komi & 4.47 & 1.56 & 0.79 & 0.58 & 0.64 & -0.43 & 0.76 & 0.29 & 0.47 & 2.91 \\
\hline Republic Mary-El & 0.02 & -4.17 & -0.69 & -0.72 & -0.09 & 0.12 & -3.48 & -0.14 & -3.34 & 4.18 \\
\hline $\begin{array}{l}\text { Republic Mor- } \\
\text { dovia }\end{array}$ & 8.12 & 0.98 & 0.32 & 0.19 & -0.30 & 0.44 & 0.66 & 0.01 & 0.65 & 7.14 \\
\hline $\begin{array}{l}\text { Republic of Sakha } \\
\text { (Yakutia) }\end{array}$ & 3.39 & -1.25 & -1.16 & -0.11 & -0.85 & -0.20 & -0.09 & 0.43 & -0.52 & 4.64 \\
\hline $\begin{array}{l}\text { Republic North } \\
\text { Ossetia (Alania) }\end{array}$ & 11.05 & 1.68 & 1.65 & 2.50 & -0.74 & -0.12 & 0.04 & 0.04 & - & 9.37 \\
\hline Republic Tatarstan & 6.81 & 2.28 & 0.63 & 0.35 & 0.13 & 0.15 & 1.65 & 0.50 & 1.15 & 4.53 \\
\hline Republic of Tyva & 6.47 & -0.10 & 0.13 & -0.26 & 0.45 & -0.06 & -0.23 & -0.23 & - & 6.57 \\
\hline
\end{tabular}




\begin{tabular}{|c|c|c|c|c|c|c|c|c|c|c|}
\hline 1 & 2 & 3 & 4 & 5 & 6 & 7 & 8 & 9 & 10 & 11 \\
\hline $\begin{array}{l}\text { Republic Khakas- } \\
\text { sia }\end{array}$ & 2.53 & 16.22 & 0.30 & 0.76 & -0.92 & 0.46 & 15.92 & -0.12 & 16.04 & -13.69 \\
\hline Rostov Oblast & 9.80 & 3.00 & 1.55 & 0.85 & 0.51 & 0.19 & 1.45 & 0.10 & 1.35 & 6.79 \\
\hline Ryazan Oblast & 5.42 & 2.00 & 1.74 & 0.60 & -0.23 & 1.37 & 0.26 & 0.04 & 0.22 & 3.42 \\
\hline Samara Oblast & 5.46 & 2.62 & 1.28 & 0.82 & 0.46 & 0.00 & 1.34 & 0.18 & 1.16 & 2.84 \\
\hline Saratov Oblast & 6.83 & 1.05 & 0.65 & 0.00 & 0.23 & 0.43 & 0.40 & 0.00 & 0.40 & 5.78 \\
\hline Sakhalin Oblast & 0.71 & 0.91 & 3.04 & 1.43 & 0.89 & 0.72 & -2.13 & 0.51 & -2.65 & -0.20 \\
\hline Sverdlovsk Oblast & 7.95 & 2.47 & 1.25 & 0.56 & 0.40 & 0.29 & 1.22 & 0.06 & 1.16 & 5.47 \\
\hline Smolensk Oblast & 3.62 & 1.88 & 0.53 & 0.39 & -0.10 & 0.25 & 1.34 & 0.09 & 1.26 & 1.74 \\
\hline Stavropol Krai & 7.39 & 0.40 & 1.20 & 0.67 & 0.56 & -0.02 & -0.80 & 0.09 & -0.89 & 6.99 \\
\hline Tambov Oblast & 7.76 & -2.23 & -0.73 & -0.58 & -0.57 & 0.43 & -1.50 & -0.22 & -1.28 & 9.99 \\
\hline Tver Oblast & 3.63 & -0.05 & 0.27 & 0.08 & -0.03 & 0.22 & -0.32 & 0.16 & -0.48 & 3.68 \\
\hline Tomsk Oblast & 9.53 & 3.44 & 1.81 & 0.55 & 0.85 & 0.42 & 1.63 & 0.13 & 1.49 & 6.09 \\
\hline Tula Oblast & 5.11 & 0.37 & 0.02 & -0.36 & 0.38 & -0.01 & 0.36 & -0.01 & 0.36 & 4.74 \\
\hline Tyumen Oblast & 7.89 & 4.52 & 1.22 & 0.60 & 0.07 & 0.55 & 3.29 & 0.90 & 2.39 & 3.38 \\
\hline $\begin{array}{l}\text { Udmurtia Repub- } \\
\text { lic }\end{array}$ & 5.54 & 2.21 & 0.71 & 0.79 & -0.02 & -0.06 & 1.50 & 0.12 & 1.38 & 3.32 \\
\hline Ulyanovsk Oblast & 3.79 & -1.21 & 0.22 & -0.12 & 0.02 & 0.32 & -1.43 & -0.18 & -1.26 & 5.01 \\
\hline Khabarovsk Krai & 8.34 & 2.43 & 2.25 & 1.61 & 0.03 & 0.61 & 0.19 & 0.19 & - & 5.90 \\
\hline $\begin{array}{l}\text { Chelyabinsk } \\
\text { Oblast }\end{array}$ & 4.63 & -0.34 & 0.68 & 0.07 & 0.60 & 0.02 & -1.03 & 0.08 & -1.11 & 4.97 \\
\hline Chita Oblast & 5.86 & 0.50 & 0.50 & 1.49 & -0.79 & -0.19 & 0.00 & 0.00 & - & 5.35 \\
\hline Chuvash Republic & 4.95 & 1.81 & 0.65 & 0.32 & 0.03 & 0.30 & 1.16 & -0.04 & 1.20 & 3.14 \\
\hline $\begin{array}{l}\text { Chukotka autono- } \\
\text { mous okrug }\end{array}$ & 21.22 & -1.19 & -1.20 & -0.72 & 1.06 & -1.54 & 0.01 & 0.01 & -5.83 & 22.42 \\
\hline Yaroslavl Oblast & 6.80 & 2.89 & 1.09 & 0.62 & 0.32 & 0.14 & 1.80 & 0.11 & 1.69 & 3.90 \\
\hline
\end{tabular}

Table 2A-14

\section{Regions' CPI and GDP deflators (December to December of the previous year; percentage)}

\begin{tabular}{cccccccc}
\hline Region & & $\mathbf{1 9 9 7}$ & $\mathbf{1 9 9 8}$ & $\mathbf{1 9 9 9}$ & $\mathbf{2 0 0 0}$ & $\mathbf{2 0 0 1}$ & $\mathbf{2 0 0 2}$ \\
\hline 1 & 2 & 3 & 4 & 5 & 6 & 7 & 8 \\
\hline \multirow{2}{*}{ Russian Federation } & CPI & 111.00 & 184.40 & 136.50 & 120.20 & 118.60 & 115.10 \\
& GDP deflator & 113.33 & 116.21 & 162.09 & 135.40 & 117.43 & 115.86 \\
\multirow{2}{*}{ Altay Krai } & CPI & 111.20 & 171.00 & 135.40 & 122.60 & 119.40 & 113.10 \\
& GDP deflator & 115.34 & 110.12 & 147.72 & 129.65 & 121.55 & 114.80
\end{tabular}




\begin{tabular}{|c|c|c|c|c|c|c|c|}
\hline 1 & 2 & 3 & 4 & 5 & 6 & 7 & 8 \\
\hline \multirow{2}{*}{ Amour Oblast } & $C P I$ & 112.00 & 171.50 & 124.00 & 118.00 & 119.70 & 112.40 \\
\hline & GDP deflator & 134.78 & 107.10 & 137.61 & 118.73 & 123.60 & 124.89 \\
\hline \multirow{2}{*}{ Arkhangel' Oblast } & $C P I$ & 107.30 & 171.80 & 132.20 & 121.00 & 119.60 & 116.10 \\
\hline & GDP deflator & 118.72 & 113.80 & 144.50 & 145.88 & 103.04 & 122.20 \\
\hline \multirow{2}{*}{ Astrakhan Oblast } & $C P I$ & 114.40 & 171.00 & 136.20 & 116.60 & 121.60 & 114.50 \\
\hline & GDP deflator & 111.12 & 117.66 & 143.87 & 155.39 & 104.82 & 117.55 \\
\hline \multirow{2}{*}{ Belgorod Oblast } & $C P I$ & 113.40 & 167.70 & 140.80 & 120.90 & 119.20 & 116.40 \\
\hline & GDP deflator & 107.76 & 118.19 & 157.64 & 116.05 & 116.45 & 119.10 \\
\hline \multirow{2}{*}{ Bryansk Oblast } & $C P I$ & 115.40 & 175.40 & 139.20 & 118.60 & 117.30 & 116.90 \\
\hline & GDP deflator & 104.40 & 105.08 & 158.89 & 126.79 & 116.38 & 122.13 \\
\hline \multirow{2}{*}{ Vladimir Oblast } & $C P I$ & 110.90 & 172.30 & 135.80 & 118.10 & 120.50 & 113.80 \\
\hline & GDP deflator & 113.59 & 113.48 & 155.02 & 119.93 & 124.46 & 114.64 \\
\hline \multirow{2}{*}{ Volgograd Oblast } & CPI & 113.40 & 171.70 & 138.00 & 119.20 & 120.50 & 117.00 \\
\hline & GDP deflator & 104.51 & 116.73 & 147.39 & 130.22 & 118.23 & 119.60 \\
\hline \multirow{2}{*}{ Vologda Oblast } & $C P I$ & 109.80 & 163.50 & 136.90 & 119.70 & 115.40 & 113.10 \\
\hline & GDP deflator & 103.91 & 126.64 & 170.67 & 140.42 & 94.51 & 119.20 \\
\hline \multirow{2}{*}{ Voronezh Oblast } & $C P I$ & 112.80 & 173.90 & 136.30 & 122.70 & 117.10 & 114.20 \\
\hline & GDP deflator & 110.73 & 111.50 & 150.99 & 125.96 & 119.56 & 135.57 \\
\hline \multirow{2}{*}{ the city of Moscow } & CPI & 112.80 & 209.60 & 146.50 & 122.10 & 120.40 & 117.20 \\
\hline & GDP deflator & 121.01 & 125.15 & 198.55 & 139.95 & 114.95 & 117.11 \\
\hline \multirow{2}{*}{$\begin{array}{l}\text { the city of Saint- } \\
\text { Petersburg }\end{array}$} & $C P I$ & 113.00 & 178.00 & 141.10 & 123.50 & 118.10 & 114.70 \\
\hline & GDP deflator & 115.80 & 128.31 & 154.22 & 123.14 & 128.33 & 113.62 \\
\hline \multirow{2}{*}{$\begin{array}{l}\text { Jewish Autonomous } \\
\text { Oblast }\end{array}$} & $C P I$ & 114.90 & 166.30 & 129.50 & 116.90 & 119.00 & 119.90 \\
\hline & GDP deflator & 131.60 & 104.68 & 184.55 & 120.85 & 115.73 & 131.55 \\
\hline \multirow{2}{*}{ Ivanovo Oblast } & $C P I$ & 109.40 & 176.30 & 139.00 & 116.60 & 119.90 & 115.60 \\
\hline & GDP deflator & 96.93 & 129.00 & 134.00 & 128.09 & 127.51 & 123.66 \\
\hline \multirow{2}{*}{ Irkutsk Oblast } & $C P I$ & 109.10 & 171.40 & 132.80 & 121.80 & 116.90 & 113.30 \\
\hline & GDP deflator & 131.58 & 108.02 & 152.46 & 124.07 & 114.76 & 117.81 \\
\hline \multirow{2}{*}{$\begin{array}{l}\text { Kabardino-Balkar Re- } \\
\text { public }\end{array}$} & $C P I$ & 116.90 & 167.80 & 135.30 & 116.80 & 122.50 & 115.60 \\
\hline & GDP deflator & 115.60 & 125.28 & 152.76 & 126.54 & 117.89 & 104.90 \\
\hline \multirow{2}{*}{ Kaliningrad Oblast } & $C P I$ & 105.50 & 202.50 & 134.50 & 117.50 & 121.00 & 109.80 \\
\hline & GDP deflator & 113.36 & 118.70 & 174.48 & 131.34 & 133.80 & 110.38 \\
\hline \multirow{2}{*}{ Kaluga Oblast } & $C P I$ & 110.40 & 173.70 & 132.70 & 118.60 & 119.60 & 117.50 \\
\hline & GDP deflator & 115.26 & 108.84 & 157.68 & 138.28 & 123.40 & 114.75 \\
\hline
\end{tabular}




\begin{tabular}{|c|c|c|c|c|c|c|c|}
\hline 1 & 2 & 3 & 4 & 5 & 6 & 7 & 8 \\
\hline \multirow{2}{*}{ Kamchatka Oblast } & $C P I$ & 113.50 & 162.30 & 151.60 & 124.30 & 115.40 & 114.10 \\
\hline & GDP deflator & 104.10 & 154.31 & 140.38 & 112.96 & 135.96 & 113.83 \\
\hline \multirow{2}{*}{$\begin{array}{l}\text { Karachay-Cherkessya } \\
\text { Republic }\end{array}$} & $C P I$ & 111.00 & 173.30 & 149.40 & 113.40 & 119.20 & 119.20 \\
\hline & GDP deflator & 111.19 & 104.91 & 163.36 & 112.73 & 117.86 & 128.43 \\
\hline \multirow{2}{*}{ Kemerovo Oblast } & $C P I$ & 108.20 & 170.60 & 126.80 & 120.30 & 118.00 & 113.90 \\
\hline & GDP deflator & 100.55 & 110.25 & 134.91 & 130.07 & 118.59 & 119.88 \\
\hline \multirow{2}{*}{ Kirov Oblast } & $C P I$ & 112.80 & 162.90 & 140.90 & 122.60 & 117.30 & 113.90 \\
\hline & GDP deflator & 115.85 & 106.96 & 153.39 & 130.48 & 116.82 & 122.53 \\
\hline \multirow{2}{*}{ Kostroma Oblast } & $C P I$ & 110.00 & 172.40 & 135.00 & 121.40 & 119.80 & 116.00 \\
\hline & GDP deflator & 118.11 & 112.57 & 152.67 & 116.52 & 130.38 & 114.88 \\
\hline \multirow{2}{*}{ KrasnodarKrai } & $C P I$ & 111.90 & 174.80 & 135.50 & 118.20 & 122.30 & 115.90 \\
\hline & GDP deflator & 105.87 & 127.12 & 165.74 & 125.39 & 131.85 & 113.51 \\
\hline \multirow{2}{*}{ Krasnoyarsk Krai } & $C P I$ & 108.80 & 160.40 & 129.90 & 121.10 & 124.30 & 116.80 \\
\hline & GDP deflator & 109.69 & 123.15 & 167.03 & 160.57 & 104.06 & 95.23 \\
\hline \multirow{2}{*}{ Kurgan Oblast } & $C P I$ & 115.90 & 173.80 & 139.20 & 119.30 & 127.80 & 113.90 \\
\hline & GDP deflator & 101.09 & 122.12 & 150.70 & 119.49 & 135.56 & 117.57 \\
\hline \multirow{2}{*}{ Kursk Oblast } & $C P I$ & 114.30 & 171.50 & 146.60 & 119.10 & 122.90 & 116.60 \\
\hline & GDP deflator & 110.33 & 114.35 & 140.69 & 122.28 & 121.59 & 111.88 \\
\hline \multirow{2}{*}{ Leningrad Oblast } & $C P I$ & 110.30 & 166.20 & 141.60 & 123.50 & 119.60 & 114.80 \\
\hline & GDP deflator & 109.35 & 126.60 & 161.34 & 126.67 & 127.04 & 107.91 \\
\hline \multirow{2}{*}{ Lipetsk Oblast } & $C P I$ & 114.50 & 161.40 & 144.80 & 121.90 & 117.10 & 113.50 \\
\hline & GDP deflator & 107.04 & 115.29 & 183.78 & 139.12 & 104.06 & 124.56 \\
\hline \multirow{2}{*}{ Magadan Oblast } & $C P I$ & 110.70 & 153.70 & 148.90 & 118.30 & 117.50 & 112.30 \\
\hline & GDP deflator & 117.51 & 132.72 & 159.17 & 119.23 & 122.25 & 126.51 \\
\hline \multirow{2}{*}{ Moscow Oblast } & $C P I$ & 107.50 & 182.50 & 134.10 & 121.80 & 121.80 & 117.80 \\
\hline & GDP deflator & 103.44 & 133.99 & 145.17 & 121.24 & 121.59 & 134.75 \\
\hline \multirow{2}{*}{ Murmansk Oblast } & $C P I$ & 114.60 & 179.30 & 135.70 & 121.90 & 122.60 & 122.40 \\
\hline & GDP deflator & 105.72 & 135.30 & 167.32 & 131.29 & 98.23 & 121.05 \\
\hline \multirow{2}{*}{ Nizhny Novgorod Oblast } & $C P I$ & 109.10 & 199.70 & 142.30 & 121.70 & 119.70 & 115.40 \\
\hline & GDP deflator & 117.59 & 105.82 & 146.82 & 130.11 & 131.39 & 116.58 \\
\hline \multirow{2}{*}{ Novgorod Oblast } & $C P I$ & 109.50 & 172.20 & 136.30 & 120.50 & 116.80 & 115.20 \\
\hline & GDP deflator & 107.52 & 121.16 & 162.48 & 128.01 & 117.10 & 114.97 \\
\hline \multirow{2}{*}{ Novosibirsk Oblast } & $C P I$ & 110.50 & 170.60 & 127.60 & 122.70 & 114.20 & 115.40 \\
\hline & GDP deflator & 121.54 & 102.88 & 146.77 & 122.54 & 124.80 & 115.97 \\
\hline
\end{tabular}




\begin{tabular}{|c|c|c|c|c|c|c|c|}
\hline 1 & 2 & 3 & 4 & 5 & 6 & 7 & 8 \\
\hline \multirow{2}{*}{ Omsk Oblast } & CPI & 111.10 & 172.20 & 131.30 & 121.30 & 115.40 & 115.20 \\
\hline & GDP deflator & 117.01 & 105.44 & 124.90 & 120.58 & 122.40 & 126.02 \\
\hline \multirow{2}{*}{ Orenburg Oblast } & CPI & 110.10 & 178.50 & 139.70 & 118.70 & 115.80 & 113.60 \\
\hline & GDP deflator & 109.40 & 112.33 & 174.90 & 133.55 & 112.65 & 106.63 \\
\hline \multirow{2}{*}{ Orel Oblast } & CPI & 106.70 & 167.90 & 139.30 & 119.00 & 116.70 & 115.10 \\
\hline & GDP deflator & 109.05 & 113.61 & 173.74 & 121.44 & 113.52 & 121.61 \\
\hline \multirow{2}{*}{ Penza Oblast } & CPI & 109.00 & 175.70 & 148.40 & 120.60 & 118.50 & 115.10 \\
\hline & GDP deflator & 102.71 & 109.07 & 153.33 & 131.30 & 122.31 & 125.10 \\
\hline \multirow{2}{*}{ Perm Oblast } & CPI & 110.50 & 183.70 & 139.10 & 124.50 & 122.50 & 116.00 \\
\hline & GDP deflator & 106.62 & 118.67 & 157.36 & 122.26 & 129.60 & 112.32 \\
\hline \multirow{2}{*}{ Primorsky Krai } & CPI & 110.10 & 171.20 & 131.80 & 119.00 & 119.10 & 113.60 \\
\hline & GDP deflator & 113.83 & 118.37 & 163.43 & 118.68 & 115.04 & 131.27 \\
\hline \multirow{2}{*}{ Pskov Oblast } & CPI & 111.70 & 168.70 & 142.20 & 118.60 & 118.40 & 112.60 \\
\hline & GDP deflator & 124.54 & 109.82 & 156.74 & 135.60 & 119.25 & 114.17 \\
\hline \multirow{2}{*}{ Republic Adygeya } & CPI & 113.50 & 168.30 & 147.00 & 119.90 & 121.50 & 114.50 \\
\hline & GDP deflator & 128.19 & 124.56 & 137.37 & 114.91 & 115.91 & 119.37 \\
\hline \multirow{2}{*}{ Altay Republic } & CPI & 113.50 & 182.40 & 134.00 & 123.60 & 113.70 & 116.40 \\
\hline & GDP deflator & 118.31 & 126.59 & 150.64 & 133.04 & 126.63 & 116.74 \\
\hline \multirow{2}{*}{ Republic Bashkortostan } & CPI & 108.70 & 178.80 & 139.20 & 121.60 & 117.40 & 114.70 \\
\hline & GDP deflator & 115.67 & 108.49 & 166.42 & 135.44 & 107.30 & 113.07 \\
\hline \multirow{2}{*}{ Republic Buryatia } & CPI & 107.90 & 163.70 & 144.60 & 120.30 & 120.90 & 110.60 \\
\hline & GDP deflator & 116.34 & 107.00 & 138.65 & 121.81 & 131.05 & 121.40 \\
\hline \multirow{2}{*}{ Republic of Dagestan } & CPI & 111.30 & 177.90 & 140.90 & 112.20 & 117.40 & 114.70 \\
\hline & GDP deflator & 142.19 & 107.03 & 153.15 & 138.79 & 127.38 & 119.23 \\
\hline \multirow{2}{*}{ Republic of Ingoushetia } & CPI & 106.90 & 172.80 & 152.30 & 122.70 & 138.70 & 117.00 \\
\hline & GDP deflator & 114.33 & 131.04 & 202.83 & 223.18 & 68.28 & 101.57 \\
\hline \multirow{2}{*}{ Republic of Kalmykia } & CPI & 117.50 & 168.50 & 144.50 & 116.90 & 119.80 & 115.20 \\
\hline & GDP deflator & 121.66 & 114.50 & 139.93 & 211.67 & 138.28 & 122.98 \\
\hline \multirow{2}{*}{ Republic Karelia } & CPI & 107.70 & 180.10 & 129.80 & 119.30 & 118.30 & 115.30 \\
\hline & GDP deflator & 116.99 & 124.20 & 160.29 & 129.09 & 116.15 & 114.59 \\
\hline \multirow{2}{*}{ Republic Komi } & CPI & 104.30 & 170.20 & 130.70 & 119.80 & 120.50 & 114.00 \\
\hline & GDP deflator & 125.50 & 116.47 & 165.97 & 125.56 & 122.36 & 112.44 \\
\hline \multirow{2}{*}{ Republic Mary-El } & CPI & 108.00 & 179.60 & 150.90 & 121.70 & 118.20 & 114.90 \\
\hline & GDP deflator & 128.77 & 111.05 & 152.89 & 124.73 & 127.46 & 119.27 \\
\hline
\end{tabular}




\begin{tabular}{|c|c|c|c|c|c|c|c|}
\hline 1 & 2 & 3 & 4 & 5 & 6 & 7 & 8 \\
\hline \multirow{2}{*}{ Republic Mordovia } & CPI & 118.70 & 174.20 & 140.90 & 128.00 & 119.70 & 118.20 \\
\hline & GDP deflator & 108.77 & 109.51 & 148.73 & 157.97 & 96.47 & 91.08 \\
\hline \multirow{2}{*}{$\begin{array}{l}\text { Republic of Sakha (Ya- } \\
\text { kutia) }\end{array}$} & $C P I$ & 106.30 & 155.40 & 137.30 & 117.40 & 113.10 & 112.10 \\
\hline & GDP deflator & 115.23 & 118.06 & 172.41 & 122.21 & 121.88 & 112.93 \\
\hline \multirow{2}{*}{$\begin{array}{l}\text { Republic North Ossetia } \\
\text { (Alania) }\end{array}$} & $C P I$ & 113.00 & 174.20 & 137.10 & 116.30 & 117.50 & 115.50 \\
\hline & GDP deflator & 112.98 & 119.14 & 165.12 & 116.46 & 132.37 & 102.23 \\
\hline \multirow{2}{*}{ Republic Tatarstan } & $C P I$ & 117.50 & 176.30 & 138.50 & 124.20 & 117.40 & 116.40 \\
\hline & GDP deflator & 110.97 & 119.66 & 153.36 & 162.44 & 97.06 & 115.47 \\
\hline \multirow{2}{*}{ Republic of Tyva } & $C P I$ & 108.40 & 154.50 & 138.50 & 122.20 & 115.00 & 109.60 \\
\hline & GDP deflator & 111.11 & 117.23 & 135.77 & 132.34 & 120.70 & 130.12 \\
\hline \multirow{2}{*}{ Republic Khakassia } & $C P I$ & 112.70 & 182.30 & 139.80 & 125.00 & 117.80 & 114.30 \\
\hline & GDP deflator & 124.73 & 114.89 & 162.86 & 121.26 & 109.78 & 127.62 \\
\hline \multirow{2}{*}{ Rostov Oblast } & CPI & 117.60 & 179.40 & 145.60 & 116.10 & 122.30 & 118.50 \\
\hline & GDP deflator & 112.01 & 117.05 & 155.37 & 123.79 & 115.16 & 112.01 \\
\hline \multirow{2}{*}{ Ryazan Oblast } & CPI & 111.90 & 179.40 & 139.30 & 118.10 & 119.20 & 112.00 \\
\hline & GDP deflator & 114.72 & 104.32 & 156.77 & 122.50 & 132.26 & 124.57 \\
\hline \multirow{2}{*}{ Samara Oblast } & $C P I$ & 112.50 & 183.20 & 132.50 & 119.90 & 116.70 & 117.20 \\
\hline & GDP deflator & 116.73 & 108.53 & 155.74 & 124.52 & 122.21 & 113.21 \\
\hline \multirow{2}{*}{ Saratov Oblast } & CPI & 115.30 & 184.10 & 138.90 & 119.00 & 119.00 & 111.80 \\
\hline & GDP deflator & 109.04 & 110.53 & 153.48 & 126.04 & 128.25 & 109.94 \\
\hline \multirow{2}{*}{ Sakhalin Oblast } & $C P I$ & 109.00 & 174.30 & 140.70 & 115.60 & 119.40 & 117.60 \\
\hline & GDP deflator & 124.95 & 114.83 & 174.99 & 153.06 & 116.52 & 110.36 \\
\hline \multirow{2}{*}{ Sverdlovsk Oblast } & $C P I$ & 110.60 & 194.50 & 135.10 & 123.90 & 120.10 & 113.70 \\
\hline & GDP deflator & 113.77 & 114.70 & 151.28 & 122.74 & 117.25 & 111.65 \\
\hline \multirow{2}{*}{ Smolensk Oblast } & $C P I$ & 113.20 & 176.70 & 144.70 & 122.50 & 116.60 & 116.10 \\
\hline & GDP deflator & 111.28 & 117.59 & 142.81 & 133.66 & 119.42 & 122.38 \\
\hline \multirow{2}{*}{ Stavropol Krai } & $C P I$ & 117.60 & 171.40 & 140.80 & 118.80 & 119.80 & 116.20 \\
\hline & GDP deflator & 119.73 & 121.83 & 133.91 & 132.48 & 113.53 & 111.72 \\
\hline \multirow{2}{*}{ Tambov Oblast } & $C P I$ & 112.80 & 169.90 & 145.10 & 120.30 & 115.50 & 114.30 \\
\hline & GDP deflator & 106.74 & 118.96 & 156.39 & 124.34 & 120.96 & 114.79 \\
\hline \multirow{2}{*}{ Tver Oblast } & $C P I$ & 113.80 & 171.10 & 139.20 & 121.90 & 119.00 & 115.00 \\
\hline & GDP deflator & 116.86 & 114.73 & 149.55 & 126.61 & 129.75 & 113.46 \\
\hline \multirow{2}{*}{ Tomsk Oblast } & $C P I$ & 111.40 & 174.70 & 137.00 & 119.20 & 123.50 & 116.70 \\
\hline & GDP deflator & 120.37 & 112.73 & 130.86 & 136.25 & 129.34 & 119.27 \\
\hline
\end{tabular}




\begin{tabular}{|c|c|c|c|c|c|c|c|}
\hline 1 & 2 & 3 & 4 & 5 & 6 & 7 & 8 \\
\hline \multirow{2}{*}{ Tula Oblast } & $C P I$ & 110.70 & 169.40 & 139.40 & 121.70 & 117.20 & 114.90 \\
\hline & GDP deflator & 113.15 & 118.44 & 162.24 & 132.29 & 122.55 & 119.42 \\
\hline \multirow{2}{*}{ Tyumen Oblast } & $C P I$ & 114.80 & 164.50 & 136.30 & 120.80 & 119.90 & 115.50 \\
\hline & GDP deflator & 109.06 & 103.67 & 165.21 & 163.90 & 120.19 & 112.00 \\
\hline \multirow{2}{*}{ Udmurtia Republic } & $C P I$ & 112.00 & 164.50 & 142.80 & 122.20 & 120.40 & 118.00 \\
\hline & GDP deflator & 106.55 & 107.93 & 182.69 & 133.15 & 118.04 & 120.65 \\
\hline \multirow{2}{*}{ Ulyanovsk Oblast } & $C P I$ & 123.10 & 163.20 & 143.40 & 123.50 & 128.10 & 118.00 \\
\hline & GDP deflator & 106.68 & 110.13 & 147.92 & 123.50 & 125.15 & 110.15 \\
\hline \multirow{2}{*}{ Khabarovsk Krai } & $C P I$ & 114.80 & 163.90 & 135.00 & 119.90 & 123.50 & 117.60 \\
\hline & GDP deflator & 123.54 & 103.31 & 128.07 & 145.47 & 111.67 & 116.49 \\
\hline \multirow{2}{*}{ Chelyabinsk Oblast } & $C P I$ & 107.50 & 189.10 & 140.00 & 123.10 & 124.80 & 112.00 \\
\hline & GDP deflator & 102.83 & 108.39 & 164.94 & 146.11 & 109.35 & 119.32 \\
\hline \multirow{2}{*}{ Chita Oblast } & $C P I$ & 109.60 & 165.80 & 142.50 & 117.50 & 119.30 & 115.50 \\
\hline & GDP deflator & 117.95 & 119.54 & 147.55 & 130.35 & 111.28 & 122.86 \\
\hline \multirow{2}{*}{ Chuvash Republic } & $C P I$ & 111.70 & 167.90 & 141.10 & 126.20 & 119.10 & 117.30 \\
\hline & GDP deflator & 113.67 & 119.81 & 147.83 & 130.74 & 124.95 & 116.40 \\
\hline \multirow{2}{*}{$\begin{array}{l}\text { Chukotka autonomous } \\
\text { okrug }\end{array}$} & $C P I$ & 123.60 & 152.10 & 167.20 & 119.70 & 110.50 & 132.70 \\
\hline & GDP deflator & 98.93 & 154.88 & 127.87 & 128.80 & 154.31 & 104.90 \\
\hline \multirow{2}{*}{ Yaroslavl Oblast } & $C P I$ & 111.80 & 172.60 & 133.10 & 120.10 & 120.80 & 116.10 \\
\hline & GDP deflator & 100.93 & 127.37 & 144.06 & 118.86 & 139.13 & 118.29 \\
\hline
\end{tabular}

Table 2A-15

\section{Results of Assessments of Remainder Growth Rates for the Period between 1997 and 1998}

\begin{tabular}{lcccccc}
\hline & $\begin{array}{c}\text { TFP (So- } \\
\text { low) }\end{array}$ & $\begin{array}{c}\text { TFP (Di- } \\
\text { rect } \\
\text { method) }\end{array}$ & $\begin{array}{c}\text { TFP (dual } \\
\text { method 1) }\end{array}$ & $\begin{array}{c}\text { TFP (dual } \\
\text { method 2) }\end{array}$ & min & max \\
\hline \multicolumn{1}{c}{1} & 2 & 3 & 4 & 5 & 6 & 7 \\
\hline Russian Federation & -1.87 & & 9.46 & -3.01 & -3.01 & 9.46 \\
Altay Krai & -8.00 & -6.23 & -3.71 & -5.51 & -8.00 & -3.71 \\
Amour Oblast & -8.05 & -4.47 & -0.34 & -2.09 & -8.05 & -0.34 \\
Arkhangel' Oblast & -0.55 & 0.10 & 5.47 & -5.34 & -5.34 & 5.47 \\
Astrakhan Oblast & 4.99 & 6.15 & & -1.47 & -1.47 & 6.15 \\
Belgorod Oblast & 2.12 & 2.49 & -7.13 & -0.68 & -7.13 & 2.49
\end{tabular}




\begin{tabular}{|c|c|c|c|c|c|c|}
\hline 1 & 2 & 3 & 4 & 5 & 6 & 7 \\
\hline Bryansk Oblast & -0.27 & 1.96 & 10.06 & -2.37 & -2.37 & 10.06 \\
\hline Vladimir Oblast & -2.14 & -0.89 & 5.87 & -1.69 & -2.14 & 5.87 \\
\hline Volgograd Oblast & -1.73 & 4.50 & 3.98 & -3.74 & -3.74 & 4.50 \\
\hline Vologda Oblast & -0.17 & -0.80 & -1.30 & -2.21 & -2.21 & -0.17 \\
\hline Voronezh Oblast & -0.21 & 1.87 & 6.16 & -2.65 & -2.65 & 6.16 \\
\hline the city of Moscow & 0.18 & -0.88 & -4.68 & -9.74 & -9.74 & 0.18 \\
\hline $\begin{array}{l}\text { the city of Saint- } \\
\text { Petersburg }\end{array}$ & -3.38 & -4.06 & 17.96 & -3.89 & -4.06 & 17.96 \\
\hline $\begin{array}{l}\text { Jewish Autonomous } \\
\text { Oblast }\end{array}$ & -9.56 & -8.98 & & -4.26 & -9.56 & -4.26 \\
\hline Ivanovo Oblast & -6.61 & -6.34 & -0.57 & -1.85 & -6.61 & -0.57 \\
\hline Irkutsk Oblast & -9.40 & -7.82 & 3.49 & -8.77 & -9.40 & 3.49 \\
\hline $\begin{array}{l}\text { Kabardino-Balkar } \\
\text { Republic }\end{array}$ & 0.33 & 2.03 & 2.11 & -5.46 & -5.46 & 2.11 \\
\hline Kaliningrad Oblast & -7.28 & -6.74 & -1.69 & -11.68 & -11.68 & -1.69 \\
\hline Kaluga Oblast & -3.66 & -3.65 & 13.88 & -0.40 & -3.66 & 13.88 \\
\hline Kamchatka Oblast & -2.86 & -1.96 & -11.95 & -12.73 & -12.73 & -1.96 \\
\hline $\begin{array}{l}\text { Karachay- } \\
\text { Cherkessya Repub- } \\
\text { lic }\end{array}$ & 0.49 & 0.28 & 5.25 & -3.12 & -3.12 & 5.25 \\
\hline Kemerovo Oblast & -4.05 & -2.87 & 1.37 & -1.98 & -4.05 & 1.37 \\
\hline Kirov Oblast & -3.68 & -3.26 & 4.92 & -2.25 & -3.68 & 4.92 \\
\hline Kostroma Oblast & -0.16 & -0.13 & & -1.74 & -1.74 & -0.13 \\
\hline KrasnodarKrai & -4.59 & -4.21 & 15.01 & -2.73 & -4.59 & 15.01 \\
\hline Krasnoyarsk Krai & -2.43 & -1.81 & -0.37 & -5.70 & -5.70 & -0.37 \\
\hline Kurgan Oblast & 0.59 & 1.39 & 2.80 & -4.44 & -4.44 & 2.80 \\
\hline Kursk Oblast & 2.03 & 1.43 & & -1.44 & -1.44 & 2.03 \\
\hline Leningrad Oblast & -3.89 & -4.17 & & -3.89 & -4.17 & -3.89 \\
\hline Lipetsk Oblast & -3.49 & 0.61 & 10.24 & -2.74 & -3.49 & 10.24 \\
\hline Magadan Oblast & -8.55 & -8.91 & & -5.04 & -8.91 & -5.04 \\
\hline Moscow Oblast & 2.68 & 2.98 & 7.90 & -7.16 & -7.16 & 7.90 \\
\hline Murmansk Oblast & 2.71 & 4.24 & & -9.14 & -9.14 & 4.24 \\
\hline $\begin{array}{l}\text { Nizhny Novgorod } \\
\text { Oblast }\end{array}$ & 0.06 & 1.16 & 13.68 & -5.61 & -5.61 & 13.68 \\
\hline Novgorod Oblast & 4.70 & 4.67 & 18.86 & -3.74 & -3.74 & 18.86 \\
\hline Novosibirsk Oblast & -3.08 & -0.01 & 4.42 & -5.09 & -5.09 & 4.42 \\
\hline Omsk Oblast & -8.21 & -5.09 & 0.07 & -1.12 & -8.21 & 0.07 \\
\hline Orenburg Oblast & -4.24 & -3.10 & 3.01 & -2.25 & -4.24 & 3.01 \\
\hline
\end{tabular}




\begin{tabular}{|c|c|c|c|c|c|c|}
\hline 1 & 2 & 3 & 4 & 5 & 6 & 7 \\
\hline Orel Oblast & 5.56 & 5.26 & 7.86 & 1.11 & 1.11 & 7.86 \\
\hline Penza Oblast & -3.92 & -2.53 & 7.65 & 3.24 & -3.92 & 7.65 \\
\hline Perm Oblast & 1.92 & 1.89 & 1.58 & -0.10 & -0.10 & 1.92 \\
\hline Primorsky Krai & -1.86 & 1.28 & 4.86 & -6.77 & -6.77 & 4.86 \\
\hline Pskov Oblast & -7.48 & -6.20 & 4.74 & -5.15 & -7.48 & 4.74 \\
\hline Republic Adygeya & -3.87 & 0.40 & 1.68 & -4.18 & -4.18 & 1.68 \\
\hline Altay Republic & -3.94 & -3.09 & 7.28 & -1.34 & -3.94 & 7.28 \\
\hline $\begin{array}{l}\text { Republic Bashkor- } \\
\text { tostan }\end{array}$ & -3.55 & -0.75 & 11.19 & -5.14 & -5.14 & 11.19 \\
\hline Republic Buryatia & 3.38 & 6.37 & -9.70 & -2.91 & -9.70 & 6.37 \\
\hline $\begin{array}{l}\text { Republic of Dages- } \\
\tan \end{array}$ & -6.30 & -6.73 & -0.35 & -0.17 & -6.73 & -0.17 \\
\hline $\begin{array}{l}\text { Republic of Ingou- } \\
\text { shetia }\end{array}$ & -9.13 & -5.05 & & -1.70 & -9.13 & -1.70 \\
\hline $\begin{array}{l}\text { Republic of Kal- } \\
\text { mykia }\end{array}$ & 3.87 & 4.99 & & -1.34 & -1.34 & 4.99 \\
\hline Republic Karelia & -1.08 & -0.31 & 5.16 & -6.50 & -6.50 & 5.16 \\
\hline Republic Komi & 0.49 & 1.30 & 11.45 & -5.95 & -5.95 & 11.45 \\
\hline Republic Mary-El & 0.25 & 1.52 & 4.63 & -2.47 & -2.47 & 4.63 \\
\hline Republic Mordovia & 0.64 & 2.15 & & -6.37 & -6.37 & 2.15 \\
\hline $\begin{array}{l}\text { Republic of Sakha } \\
\text { (Yakutia) }\end{array}$ & -1.70 & -1.29 & -8.71 & -2.51 & -8.71 & -1.29 \\
\hline $\begin{array}{l}\text { Republic North } \\
\text { Ossetia (Alania) }\end{array}$ & -1.05 & -0.18 & & -4.06 & -4.06 & -0.18 \\
\hline Republic Tatarstan & -3.16 & -0.51 & 12.22 & -4.75 & -4.75 & 12.22 \\
\hline Republic of Tyva & 1.46 & 1.75 & & -1.25 & -1.25 & 1.75 \\
\hline Republic Khakassia & -3.38 & 19.40 & 3.76 & -3.40 & -3.40 & 19.40 \\
\hline Rostov Oblast & 0.86 & 5.46 & 0.95 & -8.88 & -8.88 & 5.46 \\
\hline Ryazan Oblast & 0.29 & 1.04 & 17.75 & -0.86 & -0.86 & 17.75 \\
\hline Samara Oblast & -0.86 & 0.92 & 6.08 & -3.97 & -3.97 & 6.08 \\
\hline Saratov Oblast & -0.83 & 0.73 & 6.63 & 1.11 & -0.83 & 6.63 \\
\hline Sakhalin Oblast & 0.12 & 3.16 & 3.69 & -5.26 & -5.26 & 3.69 \\
\hline Sverdlovsk Oblast & -4.79 & -4.28 & 4.32 & -4.12 & -4.79 & 4.32 \\
\hline Smolensk Oblast & -2.68 & 1.53 & & -0.08 & -2.68 & 1.53 \\
\hline Stavropol Krai & -3.37 & -1.25 & -0.76 & -0.19 & -3.37 & -0.19 \\
\hline Tambov Oblast & 3.48 & 4.14 & 6.23 & -3.38 & -3.38 & 6.23 \\
\hline Tver Oblast & -1.36 & -1.07 & 11.60 & -4.98 & -4.98 & 11.60 \\
\hline Tomsk Oblast & -4.61 & -3.27 & 0.45 & -3.30 & -4.61 & 0.45 \\
\hline
\end{tabular}




\begin{tabular}{|c|c|c|c|c|c|c|}
\hline 1 & 2 & 3 & 4 & 5 & 6 & 7 \\
\hline Tula Oblast & -2.70 & -2.45 & 10.50 & -4.28 & -4.28 & 10.50 \\
\hline Tyumen Oblast & 0.20 & -0.17 & 5.10 & -1.84 & -1.84 & 5.10 \\
\hline Udmurtia Republic & -0.95 & -0.07 & 4.38 & -0.98 & -0.98 & 4.38 \\
\hline Ulyanovsk Oblast & -1.60 & 0.06 & 9.38 & -0.88 & -1.60 & 9.38 \\
\hline Khabarovsk Krai & -1.02 & 0.09 & 12.36 & -7.46 & -7.46 & 12.36 \\
\hline Chelyabinsk Oblast & -7.56 & -6.66 & 5.64 & -0.46 & -7.56 & 5.64 \\
\hline Chita Oblast & -7.10 & -6.26 & -3.76 & -1.62 & -7.10 & -1.62 \\
\hline Chuvash Republic & -6.10 & -5.83 & 10.04 & -0.37 & -6.10 & 10.04 \\
\hline $\begin{array}{l}\text { Chukotka autono- } \\
\text { mous okrug }\end{array}$ & -10.26 & -10.09 & - & -13.06 & -13.06 & -10.09 \\
\hline Yaroslavl Oblast & -0.94 & -0.09 & 12.32 & -2.71 & -2.71 & 12.32 \\
\hline
\end{tabular}

Table 2A-16

Results of Assessments of Remainder Growth Rates for the Period between 1999 and 2002

\begin{tabular}{lcccccc}
\hline & $\begin{array}{c}\text { TFP } \\
\text { (Solow) }\end{array}$ & $\begin{array}{c}\text { TFP } \\
\text { (Direct } \\
\text { method) }\end{array}$ & $\begin{array}{c}\text { TFP (dual } \\
\text { method 1) }\end{array}$ & $\begin{array}{c}\text { TFP (dual } \\
\text { method 2) }\end{array}$ & min & max \\
\hline \multicolumn{1}{c}{1} & 2 & 3 & 4 & 5 & 6 & 7 \\
\hline Russian Federation & 6.21 & & -0.30 & 3.29 & -0.30 & 6.21 \\
Altay Krai & 5.52 & 7.49 & 3.24 & -1.00 & -1.00 & 7.49 \\
Amour Oblast & 5.08 & 5.00 & 6.36 & 4.38 & 4.38 & 6.36 \\
Arkhangel' Oblast & 6.93 & 6.68 & 2.43 & 4.55 & 2.43 & 6.93 \\
Astrakhan Oblast & 7.70 & 8.06 & & 5.62 & 5.62 & 8.06 \\
Belgorod Oblast & 5.91 & 4.89 & 5.44 & 0.99 & 0.99 & 5.91 \\
Bryansk Oblast & 3.50 & 3.39 & -0.27 & 0.03 & -0.27 & 3.50 \\
Vladimir Oblast & 5.52 & 4.35 & 2.05 & 4.99 & 2.05 & 5.52 \\
Volgograd Oblast & 4.87 & 6.18 & -3.21 & -2.17 & -3.21 & 6.18 \\
Vologda Oblast & 4.49 & 4.24 & 3.79 & 0.85 & 0.85 & 4.49 \\
Voronezh Oblast & 3.05 & 3.34 & -3.83 & -0.61 & -3.83 & 3.34 \\
the city of Moscow & 7.37 & 4.71 & -4.32 & -6.65 & -6.65 & 7.37 \\
the city of Saint- & 8.46 & 6.45 & -5.24 & 6.07 & -5.24 & 8.46 \\
Petersburg & & & & 2.44 & 2.44 & 5.12 \\
Jewish Autonomous & 3.99 & 5.12 & 4.91 & 1.76 & 1.76 & 4.99 \\
\hline $\begin{array}{l}\text { Oblast } \\
\text { Ivanovo Oblast }\end{array}$ & 4.99 & 4.71 & 4.91 & & & \\
\hline
\end{tabular}




\begin{tabular}{|c|c|c|c|c|c|c|}
\hline 1 & 2 & 3 & 4 & 5 & 6 & 7 \\
\hline Irkutsk Oblast & 2.03 & 1.45 & 8.63 & 4.98 & 1.45 & 8.63 \\
\hline $\begin{array}{l}\text { Kabardino-Balkar } \\
\text { Republic }\end{array}$ & 8.39 & 10.44 & 2.32 & 6.27 & 2.32 & 10.44 \\
\hline Kaliningrad Oblast & 7.82 & 7.69 & -6.03 & 0.89 & -6.03 & 7.82 \\
\hline Kaluga Oblast & 4.37 & 4.08 & -2.32 & 3.87 & -2.32 & 4.37 \\
\hline Kamchatka Oblast & -2.12 & -0.43 & 8.84 & -1.44 & -2.12 & 8.84 \\
\hline $\begin{array}{l}\text { Karachay- } \\
\text { Cherkessya Repub- } \\
\text { lic }\end{array}$ & 5.42 & 6.32 & -3.93 & 5.41 & -3.93 & 6.32 \\
\hline Kemerovo Oblast & 5.62 & 5.27 & 3.11 & -0.06 & -0.06 & 5.62 \\
\hline Kirov Oblast & 1.70 & 1.85 & 2.21 & 1.96 & 1.70 & 2.21 \\
\hline Kostroma Oblast & 3.59 & 4.25 & -3.53 & 1.15 & -3.53 & 4.25 \\
\hline KrasnodarKrai & 5.59 & 4.76 & -6.09 & -1.51 & -6.09 & 5.59 \\
\hline Krasnoyarsk Krai & 4.38 & 5.00 & 4.32 & -1.09 & -1.09 & 5.00 \\
\hline Kurgan Oblast & 2.25 & 3.74 & -0.12 & -0.50 & -0.50 & 3.74 \\
\hline Kursk Oblast & 3.40 & 4.57 & -2.05 & 2.93 & -2.05 & 4.57 \\
\hline Leningrad Oblast & 10.86 & 10.70 & -8.37 & 5.70 & -8.37 & 10.86 \\
\hline Lipetsk Oblast & 4.84 & 4.75 & -7.21 & -2.34 & -7.21 & 4.84 \\
\hline Magadan Oblast & 2.96 & 4.68 & -1.26 & -0.99 & -1.26 & 4.68 \\
\hline Moscow Oblast & 4.55 & 4.59 & 3.49 & 3.71 & 3.49 & 4.59 \\
\hline Murmansk Oblast & 1.95 & 2.32 & & 1.40 & 1.40 & 2.32 \\
\hline $\begin{array}{l}\text { Nizhny Novgorod } \\
\text { Oblast }\end{array}$ & 6.11 & 5.51 & -6.76 & -1.71 & -6.76 & 6.11 \\
\hline Novgorod Oblast & 3.92 & 4.31 & -8.24 & 3.69 & -8.24 & 4.31 \\
\hline Novosibirsk Oblast & 7.48 & 7.50 & 5.11 & 0.66 & 0.66 & 7.50 \\
\hline Omsk Oblast & 6.54 & 6.30 & 6.90 & 2.65 & 2.65 & 6.90 \\
\hline Orenburg Oblast & 4.52 & 3.97 & 0.19 & -1.28 & -1.28 & 4.52 \\
\hline Orel Oblast & 5.31 & 6.49 & 2.19 & 1.72 & 1.72 & 6.49 \\
\hline Penza Oblast & 4.98 & 6.91 & 1.14 & 0.31 & 0.31 & 6.91 \\
\hline Perm Oblast & 3.95 & 4.04 & 2.36 & 0.04 & 0.04 & 4.04 \\
\hline Primorsky Krai & 1.21 & 0.45 & -3.68 & 4.11 & -3.68 & 4.11 \\
\hline Pskov Oblast & 5.23 & 6.28 & -3.60 & 0.83 & -3.60 & 6.28 \\
\hline Republic Adygeya & 2.62 & 2.32 & 1.73 & 4.98 & 1.73 & 4.98 \\
\hline Altay Republic & 4.76 & 5.57 & -1.62 & -0.56 & -1.62 & 5.57 \\
\hline $\begin{array}{l}\text { Republic Bashkor- } \\
\text { tostan }\end{array}$ & 4.15 & 4.53 & -1.86 & 1.30 & -1.86 & 4.53 \\
\hline Republic Buryatia & 5.26 & 5.97 & 4.47 & 2.13 & 2.13 & 5.97 \\
\hline
\end{tabular}




\begin{tabular}{|c|c|c|c|c|c|c|}
\hline 1 & 2 & 3 & 4 & 5 & 6 & 7 \\
\hline Republic of Dagestan & 8.85 & 10.47 & -0.83 & 0.16 & -0.83 & 10.47 \\
\hline $\begin{array}{l}\text { Republic of Ingou- } \\
\text { shetia }\end{array}$ & -1.00 & -0.22 & & -15.88 & -15.88 & -0.22 \\
\hline $\begin{array}{l}\text { Republic of Kal- } \\
\text { mykia }\end{array}$ & 7.17 & 14.75 & & -10.35 & -10.35 & 14.75 \\
\hline Republic Karelia & 5.58 & 4.34 & -0.55 & 4.28 & -0.55 & 5.58 \\
\hline Republic Komi & 1.67 & 2.39 & 0.45 & 4.30 & 0.45 & 4.30 \\
\hline Republic Mary-El & -0.55 & 3.34 & 3.18 & 1.28 & -0.55 & 3.34 \\
\hline Republic Mordovia & 6.14 & 5.59 & -2.59 & 3.25 & -2.59 & 6.14 \\
\hline $\begin{array}{l}\text { Republic of Sakha } \\
\text { (Yakutia) }\end{array}$ & 3.94 & 5.01 & 5.36 & -2.62 & -2.62 & 5.36 \\
\hline $\begin{array}{l}\text { Republic North } \\
\text { Ossetia (Alania) }\end{array}$ & 8.81 & 9.56 & & -1.46 & -1.46 & 9.56 \\
\hline Republic Tatarstan & 5.83 & 4.49 & -11.65 & 0.18 & -11.65 & 5.83 \\
\hline Republic of Tyva & 7.56 & 7.88 & & 0.76 & 0.76 & 7.88 \\
\hline Republic Khakassia & 0.81 & -11.52 & 0.72 & -4.31 & -11.52 & 0.81 \\
\hline Rostov Oblast & 8.49 & 7.45 & 3.20 & 4.80 & 3.20 & 8.49 \\
\hline Ryazan Oblast & 3.74 & 4.04 & -3.29 & 1.38 & -3.29 & 4.04 \\
\hline Samara Oblast & 3.72 & 2.81 & -6.57 & 0.98 & -6.57 & 3.72 \\
\hline Saratov Oblast & 6.25 & 6.89 & -1.98 & -0.88 & -1.98 & 6.89 \\
\hline Sakhalin Oblast & 2.96 & 5.00 & 0.25 & -1.87 & -1.87 & 5.00 \\
\hline Sverdlovsk Oblast & 5.58 & 4.77 & 1.52 & 1.88 & 1.52 & 5.58 \\
\hline Smolensk Oblast & 7.12 & 6.29 & & 8.02 & 6.29 & 8.02 \\
\hline Stavropol Krai & 5.75 & 5.33 & 8.14 & 6.09 & 5.33 & 8.14 \\
\hline Tambov Oblast & 7.15 & 8.85 & 1.00 & -0.25 & -0.25 & 8.85 \\
\hline Tver Oblast & 3.03 & 3.71 & -4.84 & 5.47 & -4.84 & 5.47 \\
\hline Tomsk Oblast & 7.29 & 6.27 & 5.51 & 0.82 & 0.82 & 7.29 \\
\hline Tula Oblast & 3.62 & 3.32 & -5.63 & 1.81 & -5.63 & 3.62 \\
\hline Tyumen Oblast & 4.68 & 1.99 & -0.51 & -2.82 & -2.82 & 4.68 \\
\hline Udmurtia Republic & 3.03 & 2.17 & 0.83 & -2.56 & -2.56 & 3.03 \\
\hline Ulyanovsk Oblast & 4.09 & 5.29 & 2.57 & 0.15 & 0.15 & 5.29 \\
\hline Khabarovsk Krai & 6.84 & 7.00 & 1.54 & 2.44 & 1.54 & 7.00 \\
\hline Chelyabinsk Oblast & 4.87 & 7.13 & -5.43 & -1.82 & -5.43 & 7.13 \\
\hline Chita Oblast & 5.22 & 6.33 & 9.87 & 2.32 & 2.32 & 9.87 \\
\hline Chuvash Republic & 2.50 & 1.91 & 2.51 & 2.01 & 1.91 & 2.51 \\
\hline $\begin{array}{l}\text { Chukotka autono- } \\
\text { mous okrug }\end{array}$ & 14.46 & 14.53 & & -7.68 & -7.68 & 14.53 \\
\hline Yaroslavl Oblast & 6.98 & 5.19 & 4.67 & 6.12 & 4.67 & 6.98 \\
\hline
\end{tabular}


Table 2A-17

Results of Assessments of labor productivity index in industry by the Regions for the Period between 1990 and $2003(1990=100)$

\begin{tabular}{|c|c|c|c|c|c|c|c|}
\hline & 1990 & 1991 & 1992 & 1993 & 1994 & 1995 & 1996 \\
\hline 1 & 2 & 3 & 4 & 5 & 6 & 7 & 8 \\
\hline Russian Federation & 100 & 93.65 & 80.69 & 71.13 & 62.93 & 66.08 & 66.17 \\
\hline Altay Krai & 100 & 111.04 & 97.66 & 84.56 & 56.95 & 62.30 & 57.39 \\
\hline Amour Oblast & 100 & 99.43 & 82.44 & 77.83 & 70.21 & 59.91 & 47.73 \\
\hline Arkhangel' Oblast & 100 & 97.55 & 87.46 & 88.30 & 77.42 & 78.73 & 77.32 \\
\hline Astrakhan Oblast & 100 & 103.36 & 105.01 & 108.59 & 87.10 & 85.28 & 67.32 \\
\hline Belgorod Oblast & 100 & 100.39 & 92.25 & 85.31 & 78.10 & 81.14 & 75.56 \\
\hline Bryansk Oblast & 100 & 93.97 & 80.46 & 69.62 & 52.75 & 53.07 & 45.36 \\
\hline Vladimir Oblast & 100 & 100.55 & 81.16 & 74.85 & 58.27 & 58.86 & 54.25 \\
\hline Volgograd Oblast & 100 & 100.67 & 87.61 & 74.03 & 54.22 & 49.60 & 42.53 \\
\hline Vologda Oblast & 100 & 96.38 & 93.00 & 80.88 & 76.22 & 79.73 & 82.64 \\
\hline Voronezh Oblast & 100 & 101.59 & 92.43 & 83.03 & 56.60 & 55.21 & 49.31 \\
\hline the city of Moscow & 100 & 96.72 & 84.10 & 68.62 & 60.71 & 52.21 & 44.88 \\
\hline the city of Saint-Petersburg & 100 & 106.69 & 93.46 & 85.64 & 61.45 & 59.66 & 48.91 \\
\hline Ivanovo Oblast & 100 & 100.58 & 69.34 & 65.54 & 48.99 & 43.21 & 40.73 \\
\hline Irkutsk Oblast & 100 & 95.72 & 85.55 & 76.40 & 71.66 & 78.38 & 66.78 \\
\hline Kabardino-Balkar Republic & 100 & 96.10 & 70.20 & 77.49 & 52.75 & 54.00 & 46.24 \\
\hline Kaliningrad Oblast & 100 & 97.85 & 86.00 & 72.48 & 53.59 & 52.43 & 48.06 \\
\hline Kaluga Oblast & 100 & 94.06 & 71.55 & 60.72 & 51.96 & 56.14 & 52.87 \\
\hline Kamchatka Oblast & 100 & 89.46 & 66.60 & 58.09 & 46.93 & 53.88 & 52.84 \\
\hline Kemerovo Oblast & 100 & 93.12 & 78.98 & 73.28 & 70.12 & 73.42 & 70.59 \\
\hline Kirov Oblast & 100 & 95.22 & 80.49 & 71.06 & 54.86 & 57.03 & 52.06 \\
\hline Kostroma Oblast & 100 & 100.97 & 76.50 & 70.85 & 53.57 & 51.07 & 43.27 \\
\hline KrasnodarKrai & 100 & 113.26 & 109.21 & 89.35 & 87.52 & 77.82 & 73.50 \\
\hline Krasnoyarsk Krai & 100 & 111.99 & 96.73 & 84.97 & 79.66 & 84.95 & 82.74 \\
\hline Kurgan Oblast & 100 & 98.55 & 79.30 & 72.20 & 56.51 & 56.83 & 52.92 \\
\hline Kursk Oblast & 100 & 98.52 & 84.32 & 78.50 & 65.50 & 74.79 & 75.86 \\
\hline Leningrad Oblast & 100 & 99.19 & 96.66 & 88.54 & 77.01 & 83.87 & 76.10 \\
\hline Lipetsk Oblast & 100 & 91.71 & 83.99 & 72.04 & 67.79 & 82.07 & 85.25 \\
\hline Magadan Oblast & 100 & 102.73 & 143.21 & 114.02 & 140.74 & 135.10 & 133.38 \\
\hline Moscow Oblast & 100 & 99.32 & 84.39 & 72.02 & 57.77 & 55.10 & 46.31 \\
\hline
\end{tabular}




\begin{tabular}{|c|c|c|c|c|c|c|c|}
\hline 1 & 2 & 3 & 4 & 5 & 6 & 7 & 8 \\
\hline Murmansk Oblast & 100 & 102.89 & 95.79 & 84.78 & 82.93 & 85.88 & 81.36 \\
\hline Nizhny Novgorod Oblast & 100 & 102.34 & 99.75 & 101.13 & 69.39 & 66.33 & 65.14 \\
\hline Novgorod Oblast & 100 & 108.19 & 100.15 & 92.43 & 81.61 & 80.39 & 82.14 \\
\hline Novosibirsk Oblast & 100 & 98.74 & 79.85 & 73.31 & 62.72 & 64.49 & 66.56 \\
\hline Omsk Oblast & 100 & 102.21 & 91.73 & 79.30 & 70.73 & 79.22 & 73.74 \\
\hline Orenburg Oblast & 100 & 105.49 & 98.55 & 86.11 & 77.07 & 85.92 & 79.76 \\
\hline Orel Oblast & 100 & 94.57 & 81.23 & 71.58 & 57.73 & 56.87 & 70.49 \\
\hline Penza Oblast & 100 & 93.33 & 79.02 & 75.00 & 55.55 & 54.61 & 41.50 \\
\hline Perm Oblast & 100 & 103.17 & 93.28 & 87.52 & 75.97 & 81.64 & 82.62 \\
\hline Primorsky Krai & 100 & 98.25 & 88.03 & 93.57 & 72.99 & 77.63 & 76.95 \\
\hline Pskov Oblast & 100 & 98.91 & 84.15 & 78.08 & 56.63 & 50.10 & 46.43 \\
\hline Republic Bashkortostan & 100 & 101.64 & 94.71 & 81.38 & 71.60 & 74.18 & 73.91 \\
\hline Republic Buryatia & 100 & 88.85 & 77.51 & 83.89 & 67.79 & 67.08 & 67.20 \\
\hline Republic of Dagestan & 100 & 87.02 & 70.93 & 62.83 & 32.39 & 26.87 & 24.58 \\
\hline Republic of Kalmykia & 100 & 90.00 & 87.23 & 78.10 & 77.57 & 62.72 & 42.30 \\
\hline Republic Karelia & 100 & 97.03 & 85.39 & 76.57 & 68.43 & 75.59 & 67.34 \\
\hline Republic Komi & 100 & 93.51 & 91.91 & 84.27 & 73.46 & 73.31 & 75.58 \\
\hline Republic Mary-El & 100 & 94.19 & 69.94 & 72.74 & 60.54 & 54.87 & 46.48 \\
\hline Republic Mordovia & 100 & 103.64 & 85.14 & 83.31 & 51.72 & 46.27 & 44.38 \\
\hline $\begin{array}{l}\text { Republic of Sakha (Ya- } \\
\text { kutia) }\end{array}$ & 100 & 99.52 & 81.91 & 83.07 & 81.92 & 88.35 & 91.70 \\
\hline $\begin{array}{l}\text { Republic North Ossetia } \\
\text { (Alania) }\end{array}$ & 100 & 107.68 & 75.05 & 65.63 & 52.03 & 57.62 & 60.30 \\
\hline Republic Tatarstan & 100 & 97.95 & 88.71 & 79.12 & 75.07 & 79.75 & 89.45 \\
\hline Republic of Tyva & 100 & 89.08 & 78.93 & 79.78 & 79.66 & 59.22 & 63.48 \\
\hline Rostov Oblast & 100 & 97.49 & 91.63 & 78.66 & 57.09 & 52.96 & 46.06 \\
\hline Ryazan Oblast & 100 & 91.58 & 76.88 & 69.91 & 59.43 & 55.36 & 44.49 \\
\hline Samara Oblast & 100 & 94.80 & 89.15 & 81.46 & 68.69 & 73.41 & 73.14 \\
\hline Saratov Oblast & 100 & 101.71 & 96.10 & 93.74 & 69.15 & 63.39 & 60.18 \\
\hline Sakhalin Oblast & 100 & 104.18 & 79.51 & 58.76 & 59.21 & 73.50 & 68.39 \\
\hline Sverdlovsk Oblast & 100 & 100.24 & 75.81 & 68.89 & 60.99 & 61.89 & 58.18 \\
\hline Smolensk Oblast & 100 & 100.09 & 83.63 & 88.36 & 76.61 & 74.76 & 71.00 \\
\hline Stavropol Krai & 100 & 114.69 & 92.82 & 84.84 & 67.13 & 87.00 & 63.68 \\
\hline Tambov Oblast & 100 & 99.58 & 88.67 & 91.41 & 77.45 & 81.21 & 71.44 \\
\hline Tver Oblast & 100 & 99.39 & 82.38 & 74.45 & 59.97 & 55.71 & 48.72 \\
\hline
\end{tabular}




\begin{tabular}{lccccccc}
\hline \multicolumn{1}{c}{1} & 2 & 3 & 4 & 5 & 6 & 7 & 8 \\
\hline Tomsk Oblast & 100 & 98.39 & 94.67 & 98.21 & 89.08 & 84.70 & 85.95 \\
Tula Oblast & 100 & 101.53 & 89.10 & 81.46 & 68.14 & 72.76 & 66.48 \\
Tyumen Oblast & 100 & 96.13 & 84.89 & 73.07 & 68.90 & 70.72 & 55.05 \\
Udmurtia Republic & 100 & 97.88 & 76.62 & 76.62 & 65.40 & 61.63 & 54.71 \\
Ulyanovsk Oblast & 100 & 104.41 & 99.09 & 99.91 & 97.09 & 82.55 & 74.70 \\
Khabarovsk Krai & 100 & 102.31 & 95.90 & 84.95 & 55.91 & 50.47 & 49.08 \\
Chelyabinsk Oblast & 100 & 104.21 & 89.97 & 77.50 & 60.49 & 58.49 & 56.46 \\
Chita Oblast & 100 & 75.62 & 60.25 & 53.33 & 42.43 & 40.05 & 37.03 \\
Chuvash Republic & 100 & 97.82 & 76.02 & 71.52 & 48.14 & 48.23 & 41.46 \\
Yaroslavl Oblast & 100 & 96.21 & 76.68 & 69.58 & 58.89 & 53.06 & 46.70 \\
\hline
\end{tabular}

\begin{tabular}{lccccccc}
\hline & $\mathbf{1 9 9 7}$ & $\mathbf{1 9 9 8}$ & $\mathbf{1 9 9 9}$ & $\mathbf{2 0 0 0}$ & $\mathbf{2 0 0 1}$ & $\mathbf{2 0 0 2}$ & $\mathbf{2 0 0 3}$ \\
\hline \multicolumn{1}{c}{1} & 9 & 10 & 11 & 12 & 13 & 14 & 15 \\
\hline Russian Federation & 74.11 & 73.94 & 81.29 & 89.43 & 92.87 & 97.35 & 105.54 \\
Altay Krai & 57.58 & 57.07 & 65.13 & 70.79 & 82.72 & 83.63 & 93.97 \\
Amour Oblast & 50.05 & 43.60 & 49.27 & 55.10 & 53.59 & 58.77 & 65.83 \\
Arkhangel' Oblast & 83.81 & 89.55 & 109.88 & 136.37 & 143.56 & 141.42 & 172.04 \\
Astrakhan Oblast & 84.76 & 99.22 & 119.12 & 131.09 & 139.98 & 151.06 & 162.30 \\
Belgorod Oblast & 85.26 & 92.14 & 108.45 & 113.54 & 123.44 & 147.54 & 153.35 \\
Bryansk Oblast & 51.90 & 55.03 & 61.62 & 72.15 & 80.37 & 88.97 & 93.14 \\
Vladimir Oblast & 62.49 & 61.22 & 72.20 & 87.83 & 97.16 & 97.67 & 102.68 \\
Volgograd Oblast & 52.86 & 49.68 & 54.64 & 59.23 & 61.90 & 64.74 & 68.38 \\
Vologda Oblast & 85.83 & 89.48 & 93.75 & 97.80 & 94.57 & 99.81 & 103.54 \\
Voronezh Oblast & 56.11 & 54.13 & 59.28 & 65.78 & 67.33 & 65.86 & 71.77 \\
the city of Moscow & 53.85 & 56.04 & 63.60 & 78.32 & 86.14 & 112.83 & 127.13 \\
the city of Saint-Petersburg & 54.74 & 60.81 & 64.70 & 80.59 & 81.42 & 107.60 & 113.93 \\
Ivanovo Oblast & 43.32 & 36.95 & 42.97 & 49.12 & 55.06 & 57.30 & 63.56 \\
Irkutsk Oblast & 61.48 & 72.29 & 78.42 & 83.75 & 83.64 & 92.54 & 94.93 \\
Kabardino-Balkar Republic & 40.74 & 47.80 & 50.63 & 61.20 & 76.12 & 80.88 & 84.69 \\
Kaliningrad Oblast & 49.21 & 49.24 & 53.33 & 62.72 & 73.20 & 80.67 & 90.27 \\
Kaluga Oblast & 57.46 & 61.61 & 69.48 & 80.07 & 91.40 & 94.61 & 104.97 \\
Kamchatka Oblast & 54.09 & 57.94 & 57.62 & 51.51 & 60.90 & 58.08 & 66.17
\end{tabular}




\begin{tabular}{|c|c|c|c|c|c|c|c|}
\hline 1 & 9 & 10 & 11 & 12 & 13 & 14 & 15 \\
\hline Kemerovo Oblast & 72.75 & 73.19 & 88.73 & 97.14 & 102.18 & 102.39 & 105.37 \\
\hline Kirov Oblast & 54.40 & 54.11 & 61.20 & 65.97 & 58.98 & 60.66 & 60.36 \\
\hline Kostroma Oblast & 52.21 & 58.13 & 61.90 & 64.60 & 65.52 & 65.32 & 69.28 \\
\hline KrasnodarKrai & 73.86 & 81.93 & 89.15 & 97.95 & 98.83 & 103.25 & 115.18 \\
\hline Krasnoyarsk Krai & 83.62 & 91.19 & 97.30 & 103.82 & 115.26 & 126.05 & 131.60 \\
\hline Kurgan Oblast & 56.86 & 56.16 & 58.91 & 62.08 & 50.70 & 49.53 & 57.88 \\
\hline Kursk Oblast & 79.17 & 84.26 & 92.35 & 96.45 & 93.46 & 96.90 & 124.90 \\
\hline Leningrad Oblast & 81.90 & 83.88 & 85.79 & 104.89 & 114.65 & 155.60 & 188.37 \\
\hline Lipetsk Oblast & 85.62 & 82.83 & 88.01 & 96.33 & 99.77 & 111.95 & 128.70 \\
\hline Magadan Oblast & 171.06 & 185.63 & 188.80 & 172.28 & 180.70 & 197.24 & 188.19 \\
\hline Moscow Oblast & 60.39 & 59.18 & 75.13 & 80.85 & 97.03 & 104.61 & 117.13 \\
\hline Murmansk Oblast & 100.40 & 104.69 & 117.13 & 121.63 & 124.66 & 122.03 & 130.26 \\
\hline Nizhny Novgorod Oblast & 73.64 & 80.02 & 84.68 & 92.69 & 90.32 & 95.40 & 100.47 \\
\hline Novgorod Oblast & 93.36 & 99.91 & 113.13 & 114.38 & 127.28 & 133.57 & 144.37 \\
\hline Novosibirsk Oblast & 71.86 & 62.73 & 77.53 & 80.41 & 85.23 & 88.79 & 96.95 \\
\hline Omsk Oblast & 70.66 & 60.86 & 63.73 & 69.87 & 78.40 & 88.27 & 103.52 \\
\hline Orenburg Oblast & 80.12 & 71.81 & 77.22 & 80.93 & 80.27 & 91.40 & 116.10 \\
\hline Orel Oblast & 76.88 & 73.14 & 76.73 & 85.17 & 101.82 & 102.90 & 113.40 \\
\hline Penza Oblast & 42.07 & 44.17 & 52.83 & 57.40 & 59.97 & 55.77 & 65.69 \\
\hline Perm Oblast & 93.42 & 92.62 & 104.01 & 114.79 & 106.53 & 103.92 & 119.55 \\
\hline Primorsky Krai & 84.49 & 75.08 & 84.17 & 84.14 & 71.40 & 73.42 & 78.10 \\
\hline Pskov Oblast & 53.51 & 48.82 & 61.77 & 67.93 & 67.11 & 74.22 & 80.10 \\
\hline Republic Bashkortostan & 74.22 & 74.48 & 80.14 & 85.80 & 93.77 & 96.37 & 106.86 \\
\hline Republic Buryatia & 72.84 & 81.19 & 88.70 & 93.08 & 107.03 & 132.36 & 146.36 \\
\hline Republic of Dagestan & 32.77 & 30.28 & 25.65 & 26.95 & 33.42 & 41.82 & 46.40 \\
\hline Republic of Kalmykia & 47.70 & 51.53 & 47.60 & 52.79 & 59.26 & 54.11 & 60.19 \\
\hline Republic Karelia & 75.22 & 80.14 & 93.77 & 95.12 & 95.62 & 97.99 & 97.49 \\
\hline Republic Komi & 80.07 & 83.41 & 85.14 & 92.06 & 99.04 & 102.60 & 105.63 \\
\hline Republic Mary-El & 60.04 & 64.05 & 62.66 & 59.85 & 61.13 & 73.50 & 84.46 \\
\hline Republic Mordovia & 48.38 & 48.89 & 56.58 & 67.73 & 74.10 & 81.92 & 102.15 \\
\hline $\begin{array}{l}\text { Republic of Sakha (Ya- } \\
\text { kutia) }\end{array}$ & 87.97 & 103.41 & 101.68 & 108.61 & 100.88 & 96.95 & 100.55 \\
\hline $\begin{array}{l}\text { Republic North Ossetia } \\
\text { (Alania) }\end{array}$ & 63.36 & 74.61 & 85.66 & 90.96 & 95.73 & 96.53 & 97.13 \\
\hline Republic Tatarstan & 96.49 & 104.27 & 112.90 & 117.31 & 123.71 & 125.60 & 144.61 \\
\hline
\end{tabular}




\begin{tabular}{lccccccc}
\hline \multicolumn{1}{c}{1} & 9 & 10 & 11 & 12 & 13 & 14 & 15 \\
\hline Republic of Tyva & 52.28 & 58.62 & 69.36 & 77.40 & 75.62 & 81.20 & 81.31 \\
Rostov Oblast & 53.80 & 59.91 & 79.88 & 95.54 & 122.60 & 126.79 & 139.49 \\
Ryazan Oblast & 51.77 & 55.10 & 64.17 & 68.80 & 75.06 & 73.55 & 75.76 \\
Samara Oblast & 85.43 & 79.79 & 95.29 & 103.04 & 113.16 & 111.77 & 117.42 \\
Saratov Oblast & 65.89 & 70.80 & 80.68 & 94.72 & 103.02 & 110.21 & 126.41 \\
Sakhalin Oblast & 79.36 & 77.72 & 86.24 & 92.15 & 103.57 & 98.59 & 108.81 \\
Sverdlovsk Oblast & 58.03 & 53.78 & 59.29 & 66.49 & 72.86 & 76.24 & 83.57 \\
Smolensk Oblast & 75.55 & 75.21 & 84.80 & 95.46 & 106.06 & 108.44 & 114.45 \\
Stavropol Krai & 57.68 & 62.00 & 77.01 & 81.88 & 99.51 & 104.85 & 118.69 \\
Tambov Oblast & 78.57 & 85.81 & 101.19 & 104.94 & 110.93 & 115.80 & 122.94 \\
Tver Oblast & 50.85 & 59.04 & 64.61 & 69.14 & 72.08 & 76.89 & 88.86 \\
Tomsk Oblast & 83.64 & 84.76 & 97.65 & 101.30 & 114.32 & 121.63 & 146.61 \\
Tula Oblast & 74.40 & 77.45 & 83.76 & 95.97 & 104.95 & 110.87 & 115.95 \\
Tyumen Oblast & 59.56 & 62.15 & 60.70 & 66.11 & 55.15 & 59.21 & 66.04 \\
Udmurtia Republic & 60.03 & 62.92 & 67.01 & 76.32 & 70.14 & 75.73 & 82.44 \\
Ulyanovsk Oblast & 92.61 & 99.83 & 110.14 & 123.08 & 120.11 & 132.06 & 143.12 \\
Khabarovsk Krai & 51.62 & 61.69 & 61.08 & 72.05 & 79.06 & 83.53 & 89.70 \\
Chelyabinsk Oblast & 62.02 & 55.69 & 62.21 & 70.26 & 71.11 & 72.75 & 77.30 \\
Chita Oblast & 38.17 & 37.53 & 43.46 & 47.35 & 50.33 & 48.75 & 51.14 \\
Chuvash Republic & 45.30 & 45.20 & 48.69 & 51.75 & 54.15 & 56.76 & 59.16 \\
Yaroslavl Oblast & 52.51 & 51.03 & 62.88 & 70.62 & 77.81 & 81.74 & 88.10 \\
\hline
\end{tabular}




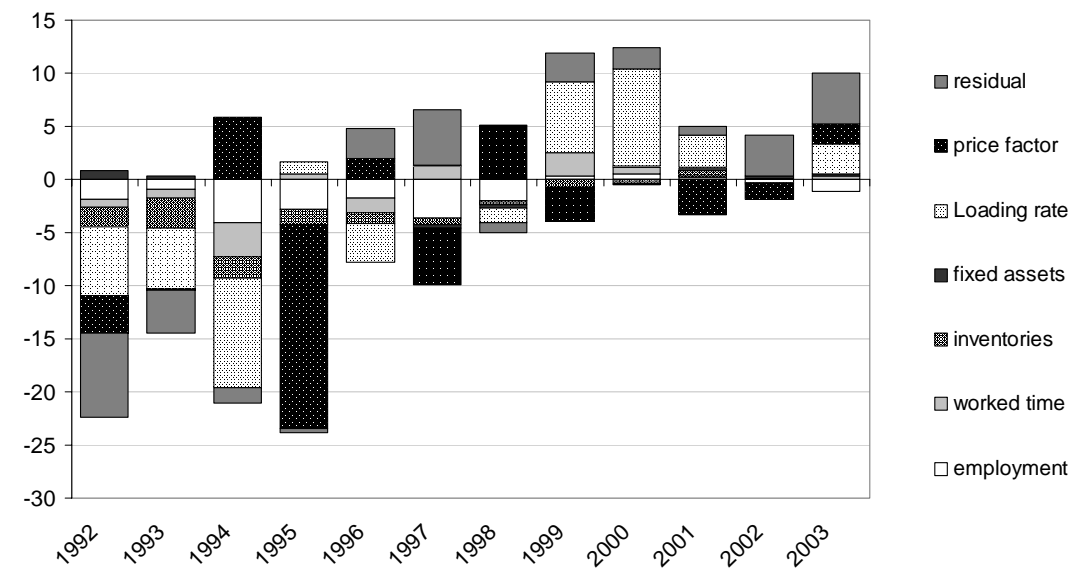

Fig. 2A-1. The Structure of GVA Growth Rates in Industry in 1992-2003

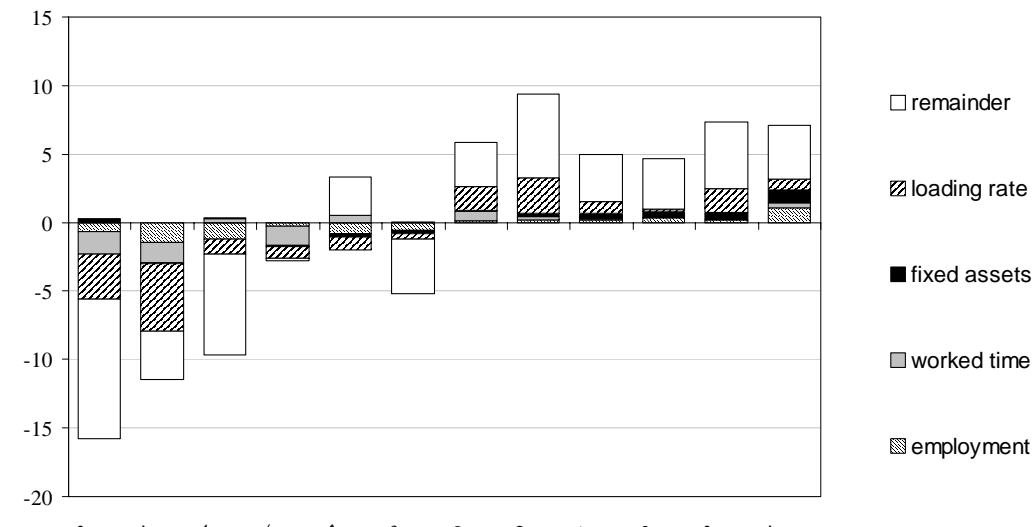

$2^{9^{3}} \quad 9^{9^{x}} \quad 9^{9^{5}} \quad 9^{9^{6}} \quad 9^{9^{1}} \quad 9^{9^{0}} \quad 9^{9^{9}} \quad 2^{0^{0}} \quad 2^{0^{2}} \quad 2^{0^{2}} \quad 2^{0^{3}} \quad 2^{0^{0}}$

Fig. 2A-2. The Structure of GDP Growth Rates in 1992-2003 


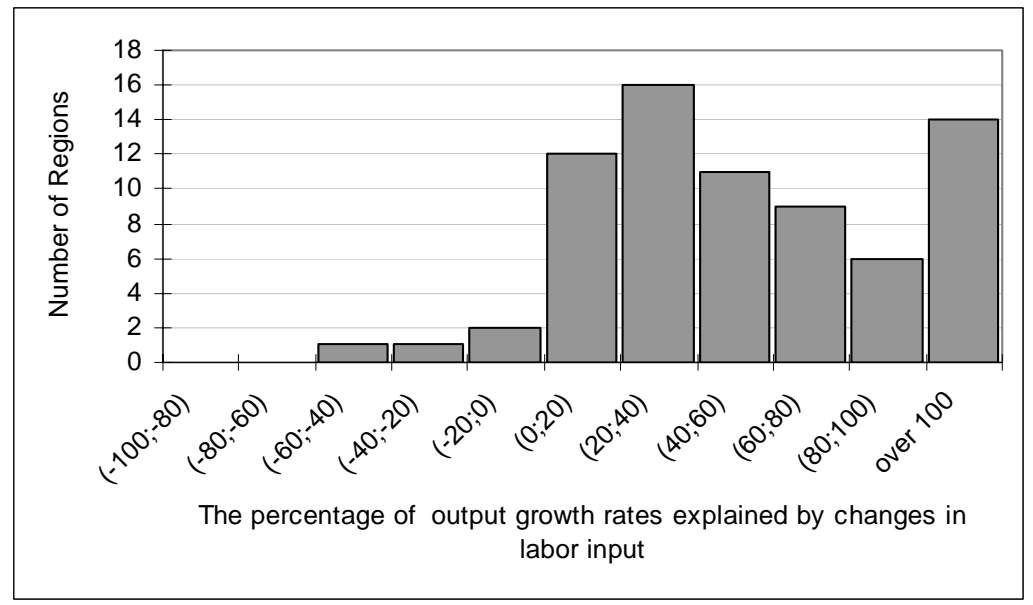

Fig. 2A-3. Bar Chart of the Percentage of the Output Growth Rates Explained by Changes in the Average Number of Employed in Regions with Account of Sectoral Differences (for the Period of 1997-1998)

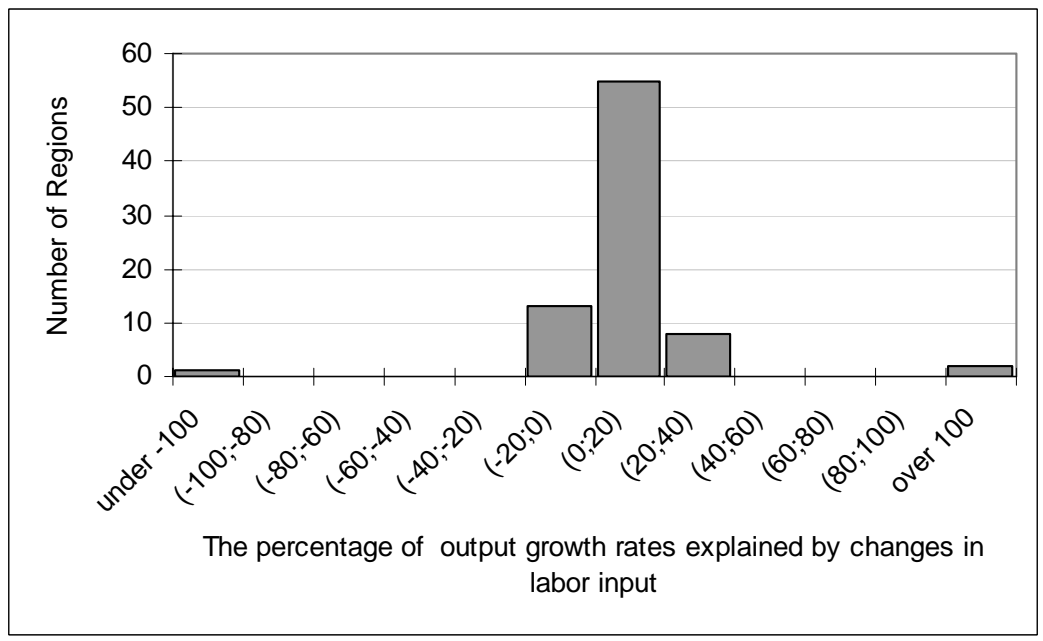

Fig. 2A-4. Bar Chart of the Percentage of the Output Growth Rates Explained by Changes in the Average Number of Employed in Regions with Account of Sectoral Differences (for the Period of 1999-2002) 


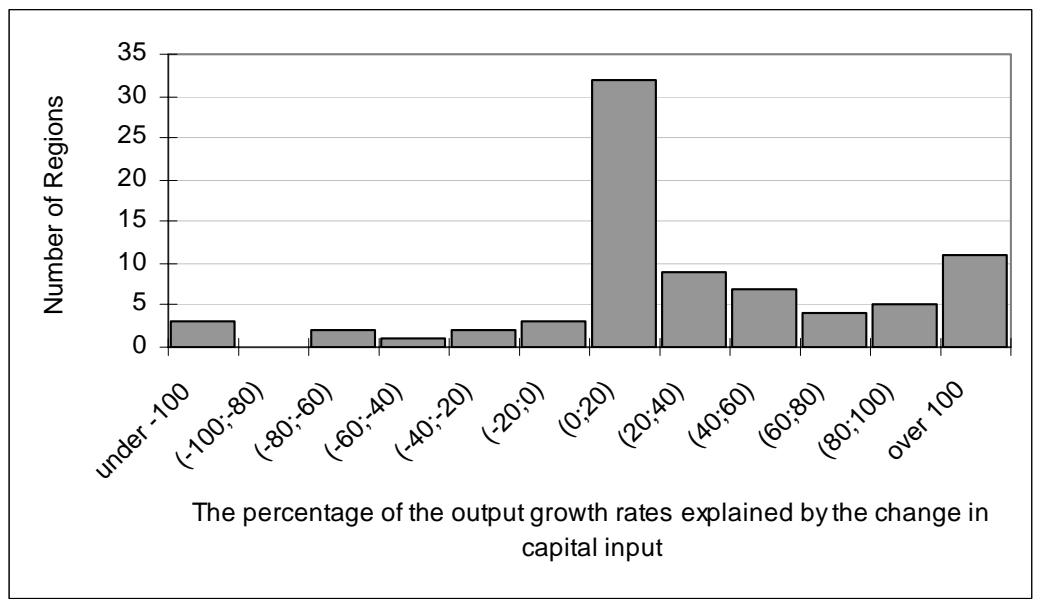

Fig. 2A-5. Bar Chart of the Output Growth Rate Explained by Changes in Capital Input Across Regions (the Period between 1997 and 1998)

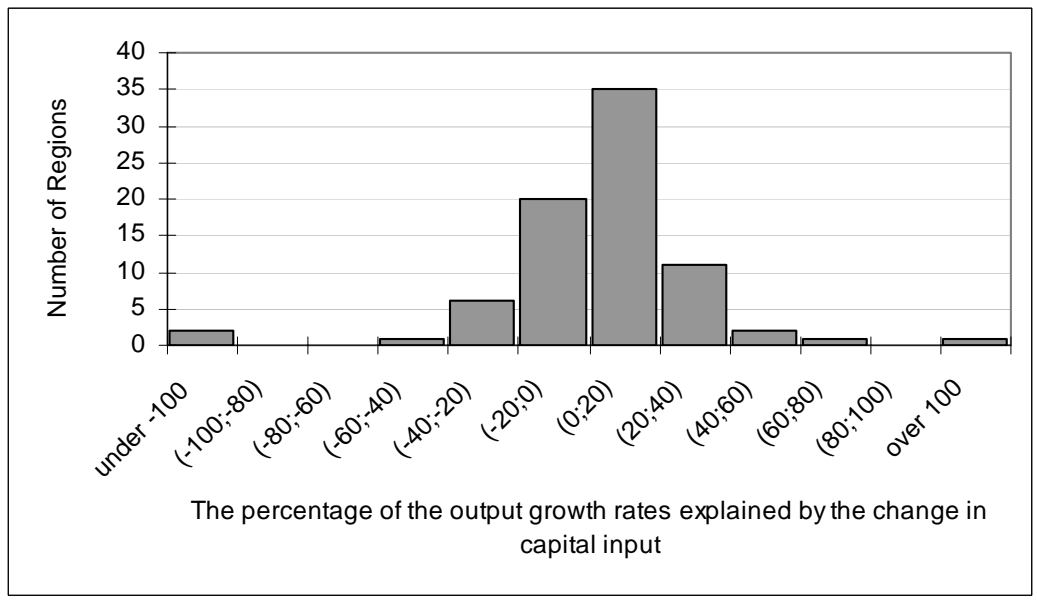

Fig. 2A-6. Bar Chart of the Output Growth Rate Explained by Changes in Capital Input Across Regions (the Period between 1999 and 2002) 


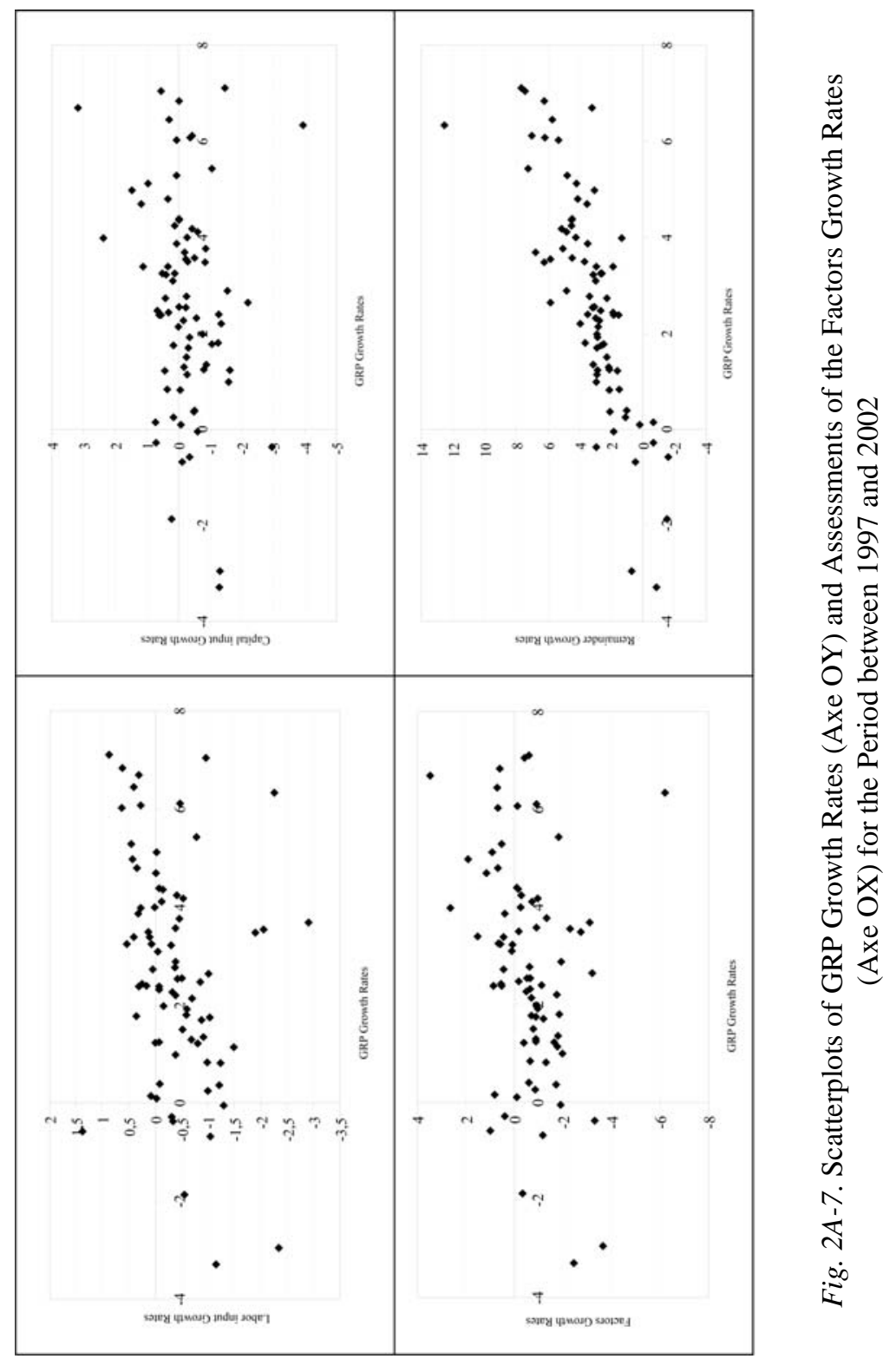




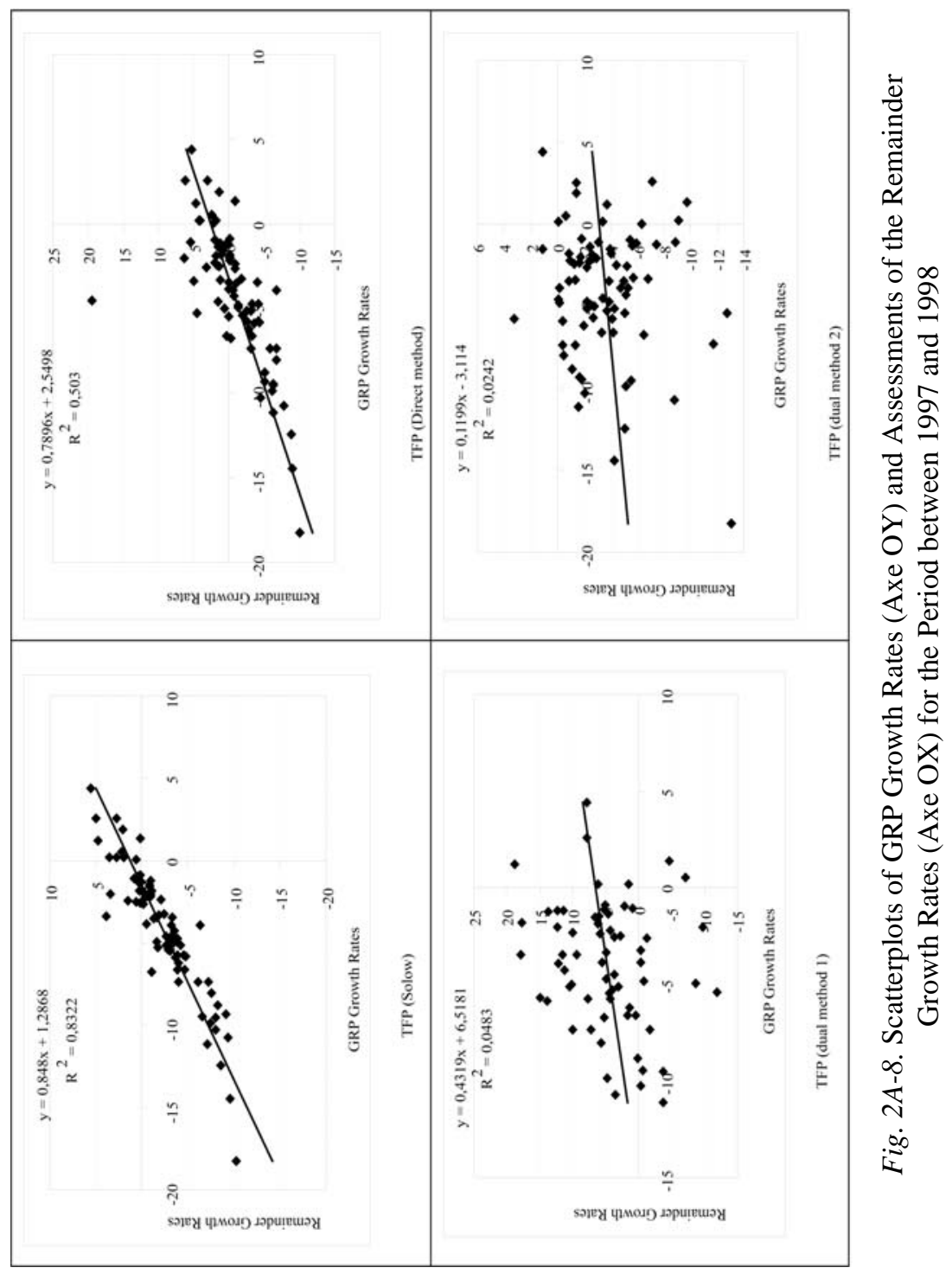




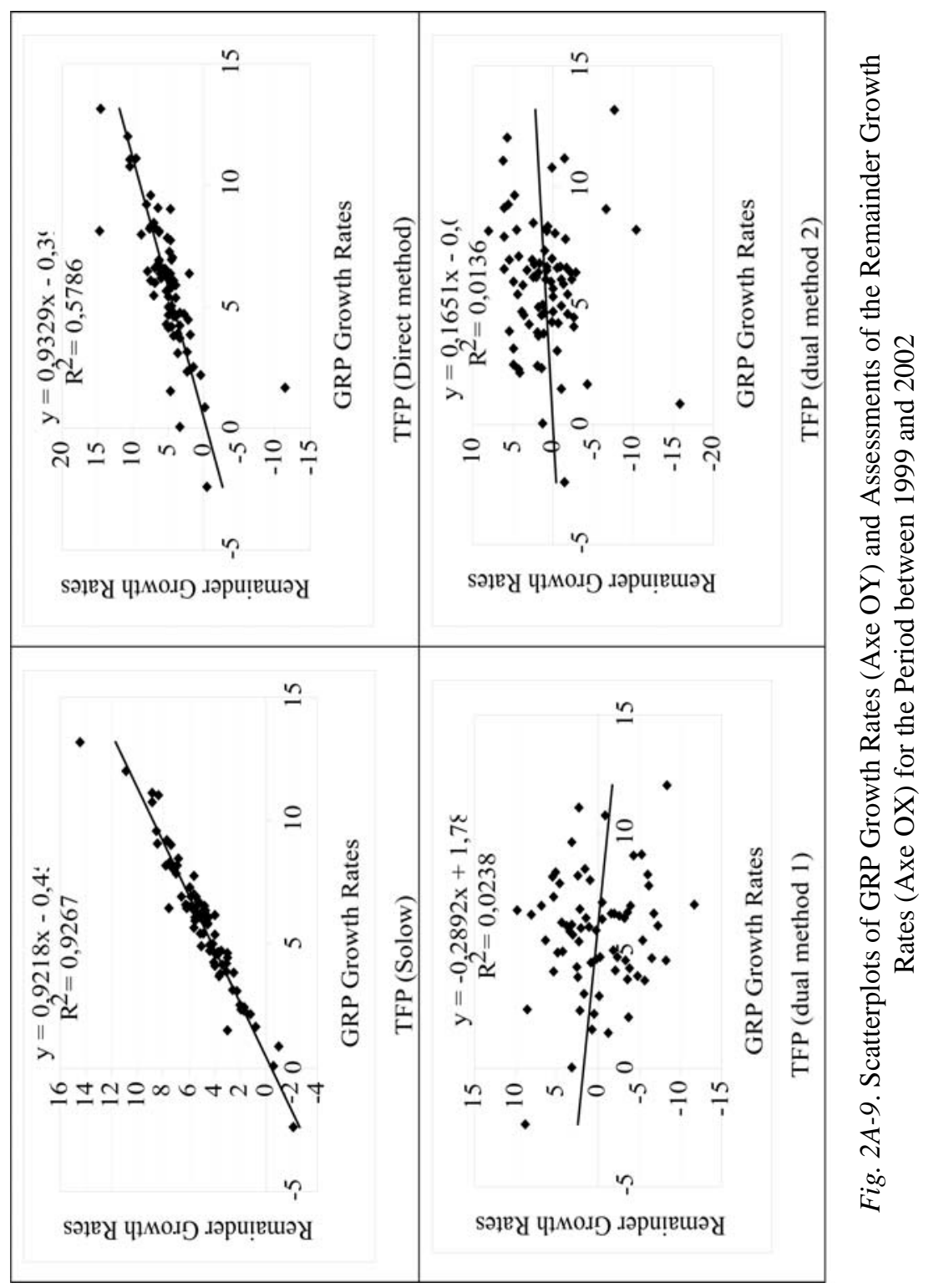




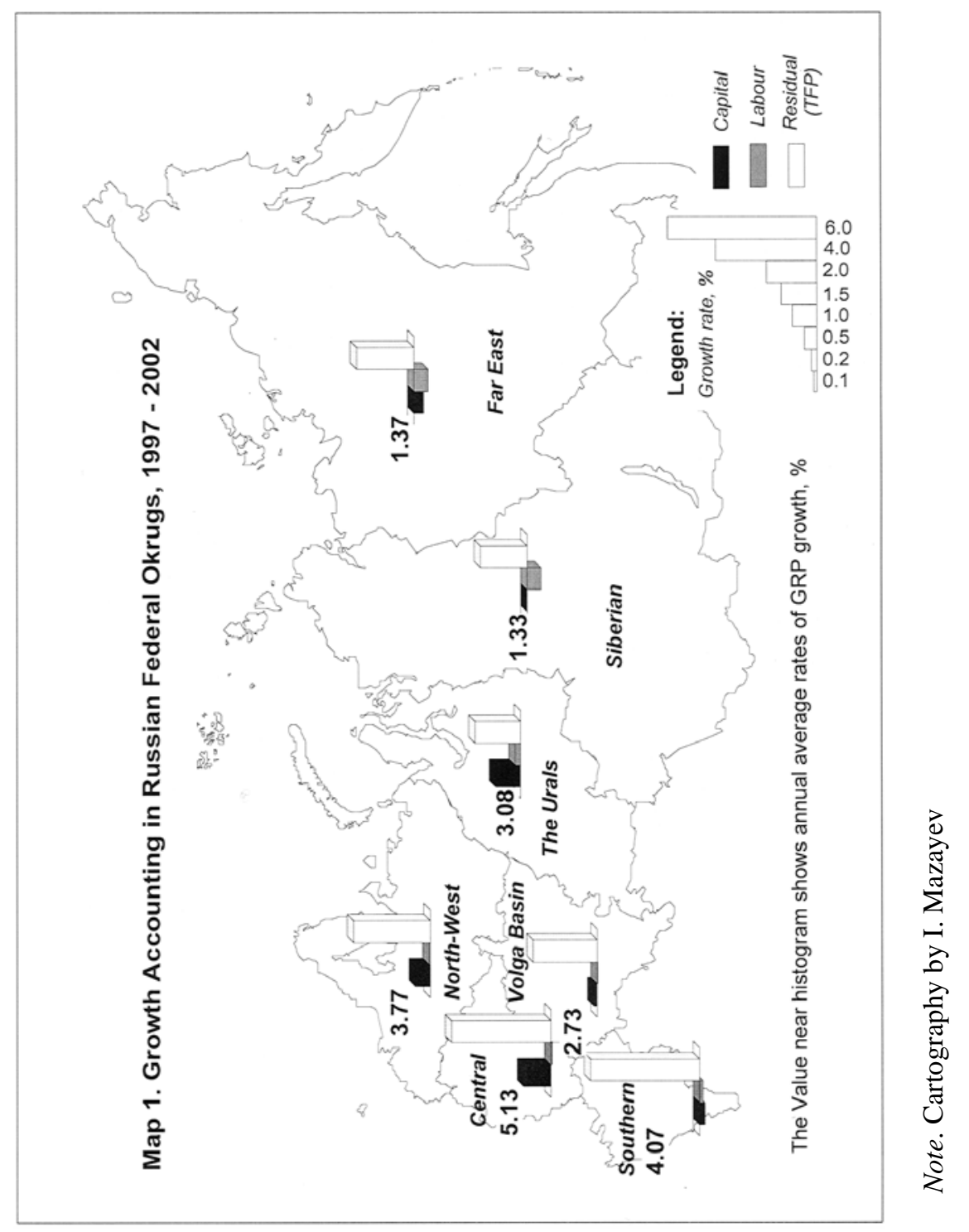




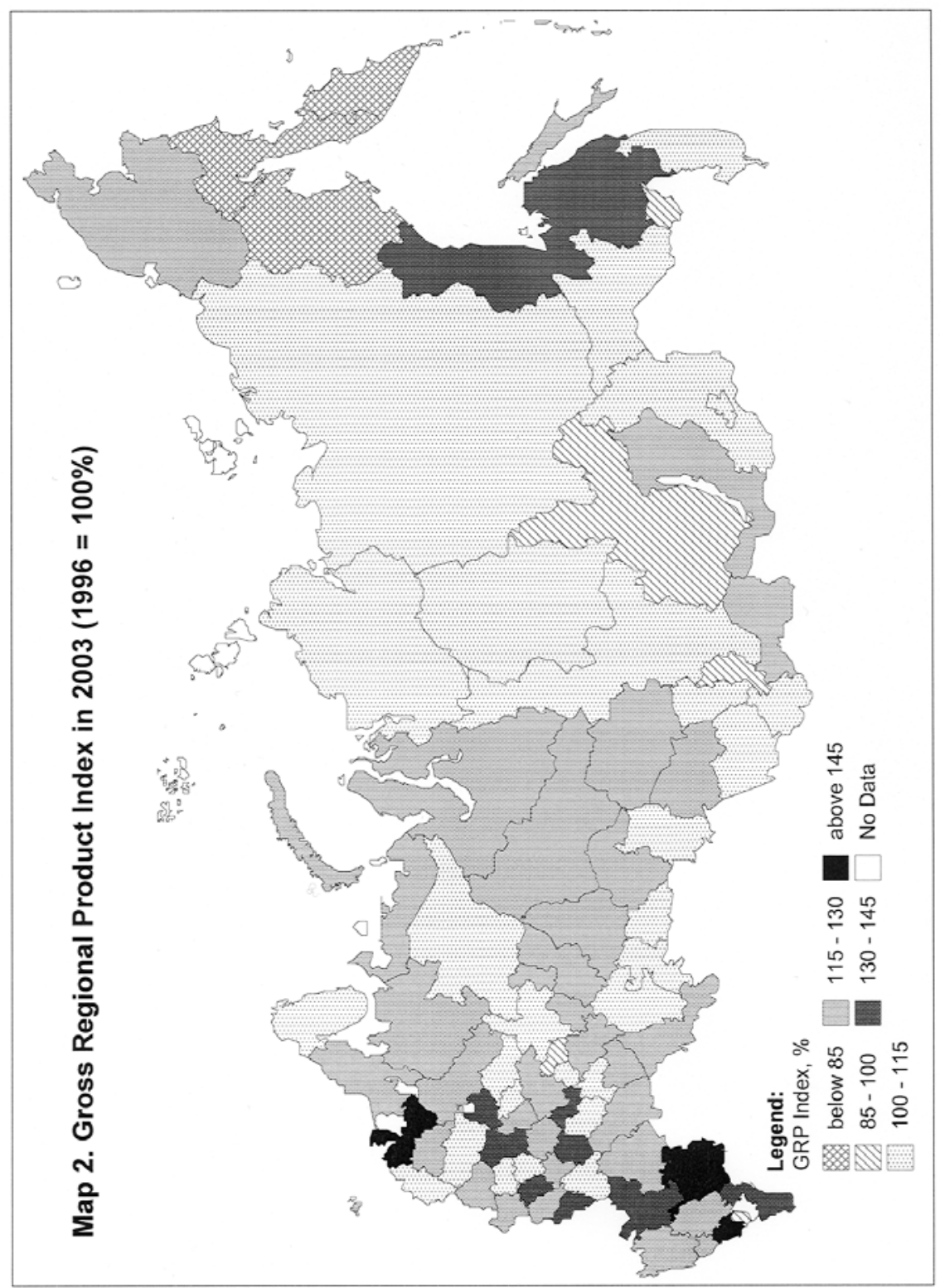




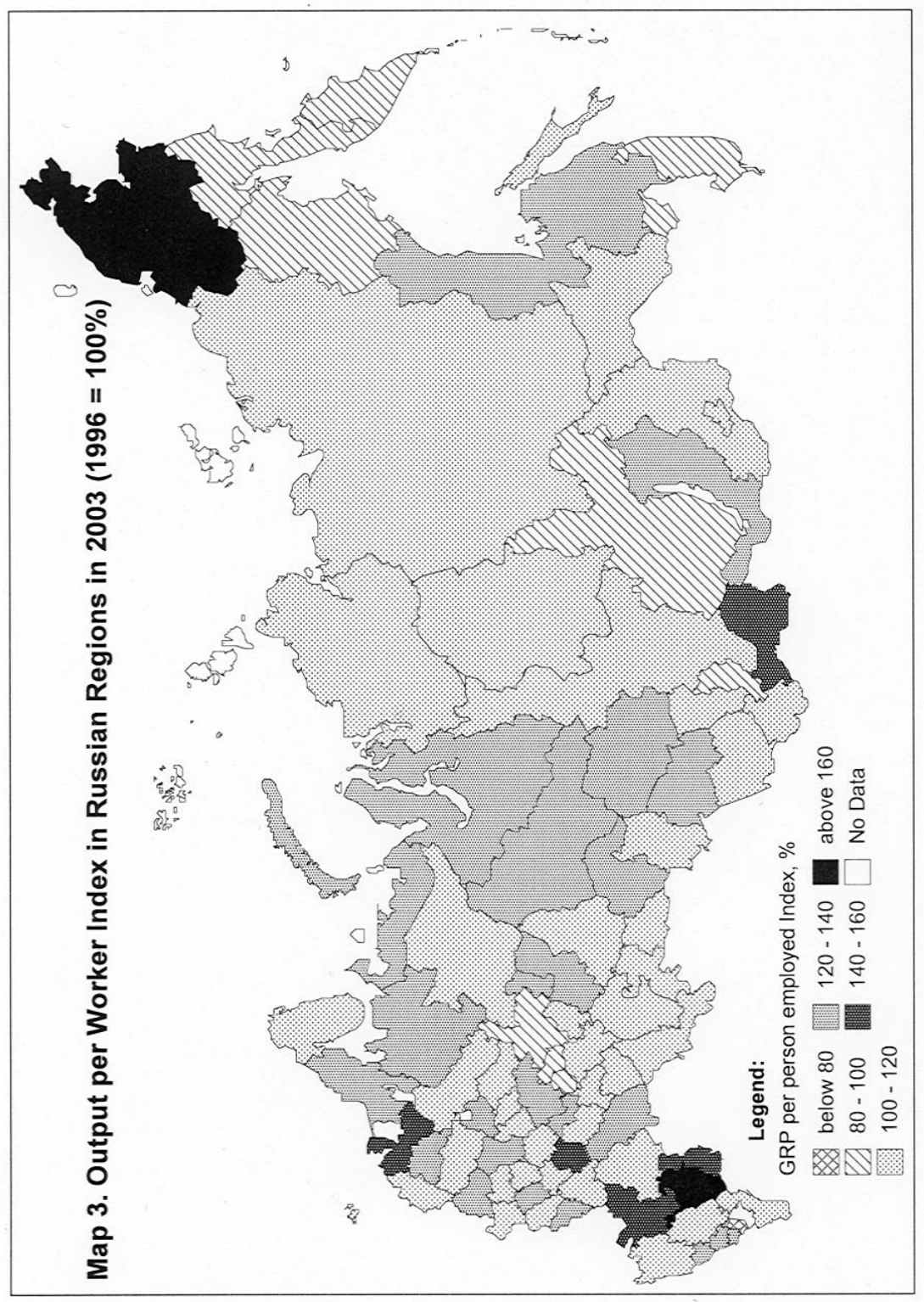




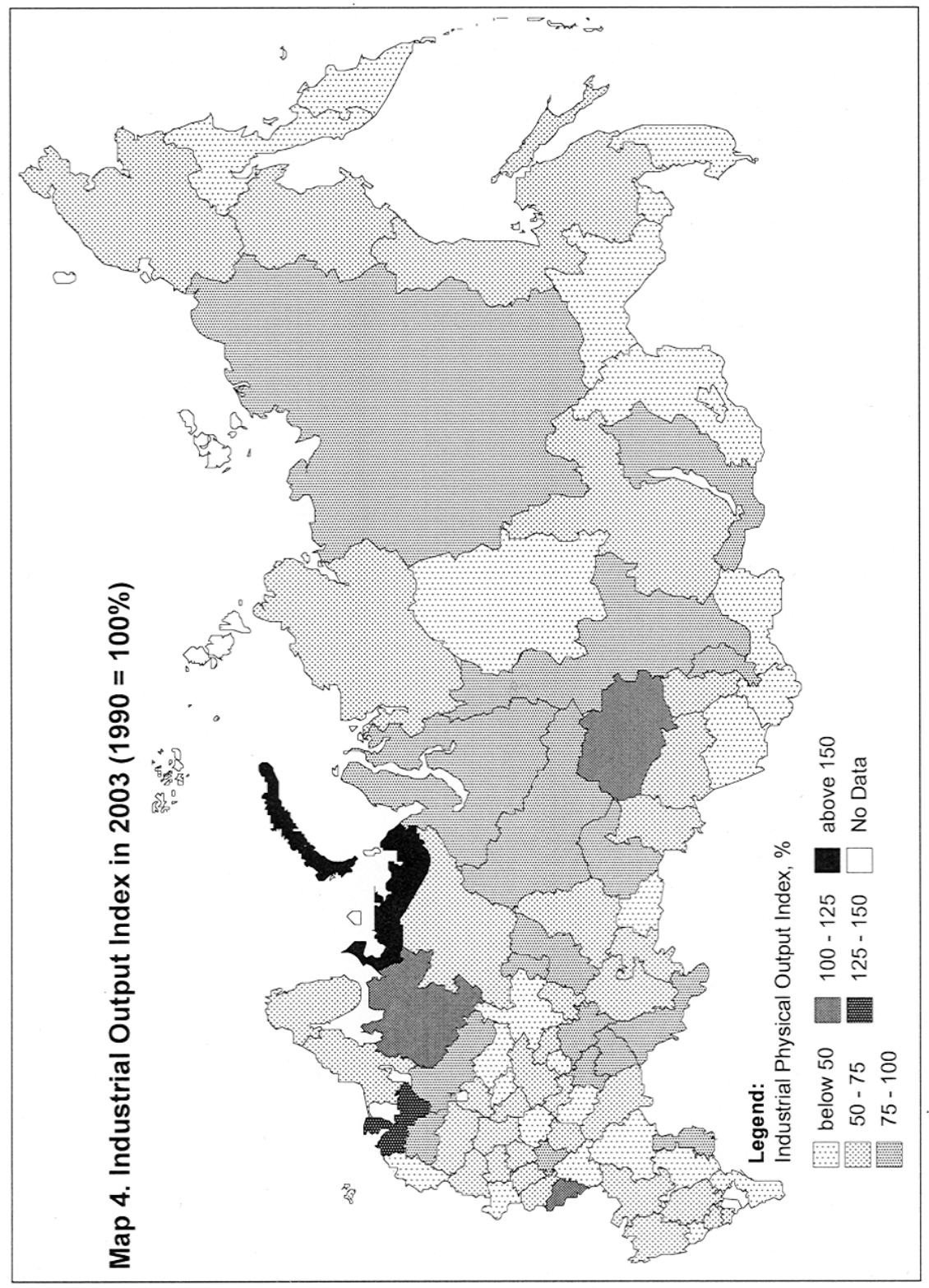




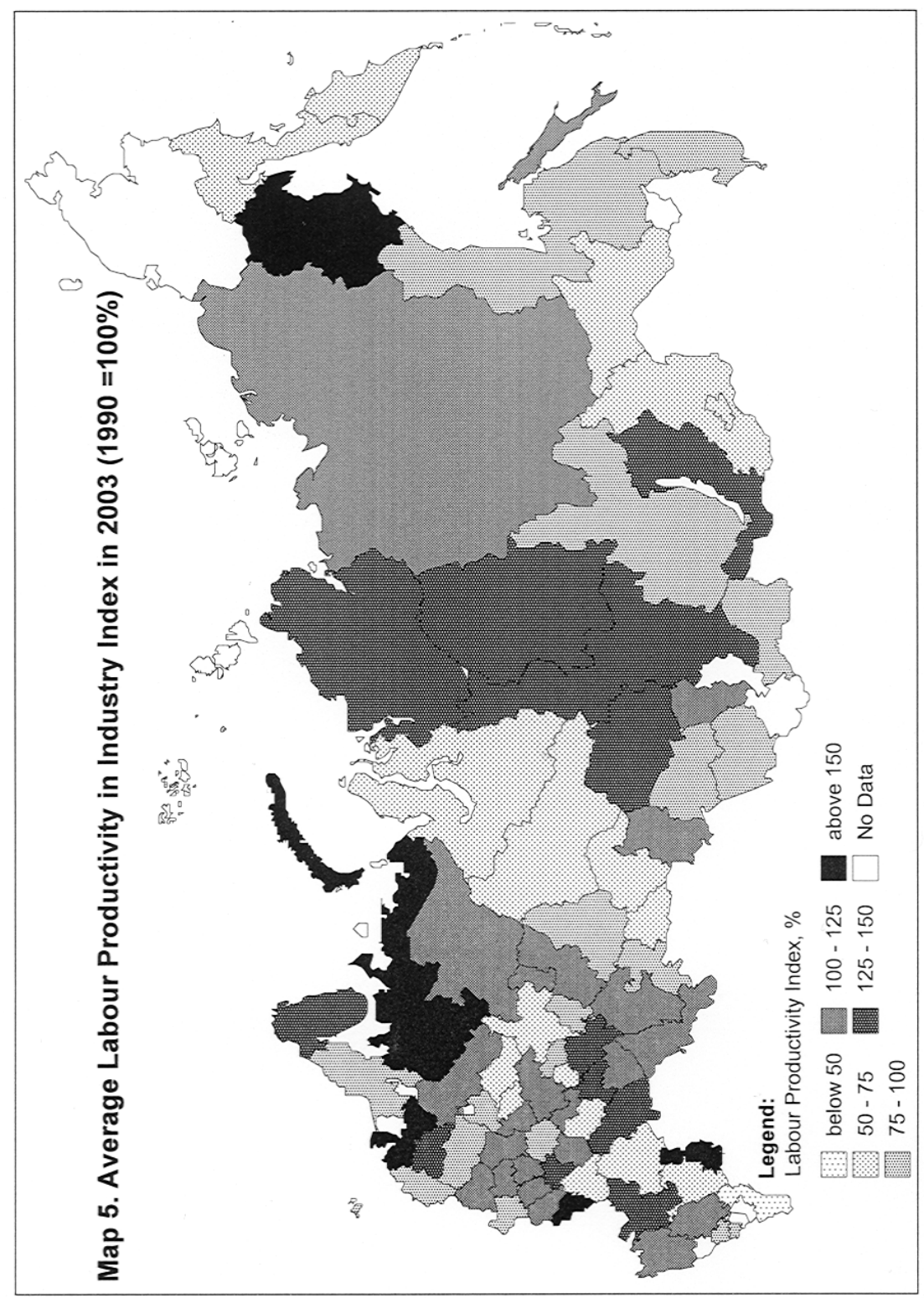




\section{Economic Growth in Regions. Relevant Canadian Experience}

While the "Economic Geography of Canada" defines and describes some 68 "economic regions" of Canada. for most purposes, the breakdown is by groupings of the 10 Provinces and 3 Territories:

- Atlantic Canada (Newfoundland, Nova Scotia, Prince Edward Island, New Brunswick),

- Quebec (Part of Central Canada, usually classed on its own),

- Ontario (Part of Central Canada, usually classed on its own),

- Prairies (Originally 3, Now more usually 2: Manitoba, Saskatchewan),

- Alberta (Originally classed as "Prairie", Now more usually classed on its own),

- British Columbia,

- The North (The 3 Territories: Yukon, Nunavut, Northwest Territories).

Canada's economic development generally can be seen in several "phases":

- Resource Extraction.

- Agriculture \& Resource Processing.

- Industrialization.

- "Services” (“Knowledge Work”).

These phases in economic development roughly track Canada's progress towards self-government and independence from Britain. To simplify, in the beginning Canada provided raw resources (fish, furs) and the Imperial centre provided finished (higher value per item) goods; later, more and more, further processing was to occur in Canada. 
However, much of this "finishing" was to occur in Central Canada, with other Regions (in their eyes, and those of a number of observers) relegated to suppliers of raw materials.

This theme of industrializing Central Canada, with the other Regions concentrating on providing raw materials to, and markets for, Central Canada is dealt with in more detail under the heading "National Policy".

Briefly, one way of seeing Canada's political-economic history is progress towards the development of the northern half of North America with all of its resources and opportunities; and at the same time remaining in "balance" with the inevitable - and over this time growing - power and influence of, and integration with, the US.

And, as indicated above, part of the Canadian balancing act over time has been to achieve independence from Britain.

\subsection{Capital Imports -Human and Financial}

It could be rightly said that Britain began its Canadian adventure in earnest 1760/63 (on the base of some 10,000 in its small colonies in Newfoundland, Nova Scotia, PEI, and New Brunswick) plus the sparsely inhabited northern Hudson's Bay Company Lands (known as Rupert's Land) with some 60,000 settlers - recently subjects of France, augmented by some British settlers, and soon to be augmented by some 40,000 Loyalist refugees from the American Revolution (with Britain losing millions of former subjects in its original Thirteen Colonies). So, some 110,000 plus or minus to hold, occupy and develop a vast space - with little or no capital, and an Imperial system that preferred to keep colonies supplying raw materials to, and buying manufactured good from the Mother Country. 
Accordingly, the critical themes in Canada's development over the next years, and indeed, continuing to this day, have been:

I) Immigration,

II) Investment.

\subsection{Federal "National Policy"}

Although the St Lawrence River-Great Lakes System constitutes one of the biggest water systems in the world, and an east-west highway to the centre of North America, with some exceptions, natural trade routes have tended to be North-South: the Atlantic provinces to New England and the Caribbean (and Europe), Quebec and Ontario to New York and the US Mid-West, the Prairies to the US West, BC to California (and the Far East). This was enhanced by the Erie Canal ensuring that western grain would be shipped via New York rather than Montreal; and by the US as a source of investment capital. As well, the surfacing from time to time of both Canada-US annexation and reciprocity movements has tended to reinforce this. The "National Policy" (below) was intended as a an East-West counterbalance.

In substance, the National Policy was composed of several basic parts: a) western resources, b) eastern manufacturing, c) protective tariffs, d) bound together by a national railroad. This was supplemented by western settlement, the necessary immigration to support it, and associated development and transportation and financing.

\subsection{US-Focus - Reciprocity / Free Trade / Continentalism}

The phases of "Colony-British Empire" and "National Policy" having passed, and the flirtations that characterized parts of the 1960s and 1970s with more European and more Pacific Trade having - in a sense - passed too; Canada has found itself with the reality of greater and greater trade and economic ties with the US. The Auto-Pact entered into with the US proved extremely beneficial to 
Canada - especially Ontario.This is now reflected by the NAFTA arrangement (Canada-US-Mexico, which quickly followed on the earlier Canada-US arrangement), likely to be followed by a Free Trade Area of the Americas.

Accordingly, it is now clear that Canada is not (and cannot be) a "stand-alone", and that its Regions not only relate to each other and to the National Government but also to the broader US, Continental and indeed Hemispheric framework. (The "global village" will be addressed later.)

The theme of Continental Security - for economic as well as military matters - is now front and centre, with a clear emphasis on development in critical regions, especially the energy-rich west.

\subsection{Resource-based Growth: Extraction and Processing}

As described by Bank of Canada Technical Report 51 (more fully dealt with below):

"The export-base or staples approach treats the demand for regional exports as the principal determinant of economic growth. Better known in Canada as the staples thesis, it was developed by Canadian economists H.A. Innis and W.A. Mackintosh. The staples thesis views economic growth as the result of the exploitation of a series of staple products, where staples are defined to be export goods with a high natural resource content. Though the thesis was initially intended to explain the development of Canada, it applies equally well to regions.

"According to the staples thesis, growth begins with the emergence of foreign demand for a staple product. This foreign demand may arise as the result of a change in tastes or because new technological developments have led to a reduction in the staple good's cost of production. Generally, it is assumed that the region is initially short of the capital and labour necessary to develop the staple; however, the high returns to developing the staple will raise the 
returns to the capital and labour employed in its production, prompting the inflow of both factors from other regions.

"The extent of the economic growth arising from staple production will depend on the strength of various types of linkages. First, there are backward linkages, which involve the development of local industries that supply inputs for the staple's production. The need for transport facilities has proved to be one of the strongest backward linkages. Next, forward linkages involve the development of secondary industries that use the staple product as an input. Such industries increase the value-added of regional exports. Third, there are lateral linkages, which according to Marr and Patterson (1980) occur 'mostly in the form of external economies generated by the export and related industries which stimulated the growth of some third industry.' Examples would be the development of a transportation network or the growth of a pool of skilled labour within the region. These types of external economies reduce costs not only for the staple industry but also for other industries, and this may attract new businesses to the region.

"Last, there are final-demand linkages, which are really a type of backward linkage. The term refers to the growth of industries that supply consumer goods and services to the labour employed in the expanding staple industry. As the local labour force grows, so will the local market for goods and services, and it will become more efficient for the region to produce these goods locally than to import them from elsewhere. The growth of these industries will in turn stimulate the growth of the regional economy” (pp. 34-5).

Another author (Watkins) put the Staples Thesis this way:

"A theory asserting that the export of natural resources, or staples, from Canada to more advanced economies has a pervasive impact on the economy as well as on the social and political systems. Furthermore, different staples (fur, fish, timber, grain, oil, etc.) have differing impacts on rates of settlement, federal- 
provincial conflicts, etc. The thesis was formulated in the 1920s by economic historians Harold A. Innis and W.A. Mackintosh. Agreeing that Canada had been born with a staple economy, they differed insofar as Mackintosh saw a continuing evolution toward a mature industrialized economy based on staple production, whereas Innis saw a tendency for Canada to become permanently locked into dependency as a resource hinterland. Contemporary proponents of the thesis argue that Innis' version more accurately describes the Canadian situation to the present. The thesis may be the most important single contribution to scholarship by Canadian social scientists and historians; it has also had some influence internationally, notably in the analysis of a comparable country such as Australia”.

Bank of Canada Working Paper 96-12 (Lefebvre \& Poloz) "The Commodity-Price Cycle and Regional Economic Performance in Canada www.bank-banque-canada.ca addresses regional development and convergence of regions by looking at commodities. The Abstract, referring to the period since the early 1970s, gives the following summary:

"We believe that, to a significant degree, regional diversity in economic performance reflects movements in Canada's terms of trade, which very frequently are tied to developments in world commodity markets. To state our hypothesis briefly, an improvement in Canada's terms of trade due to a rise in world commodity prices tends to boost output in the regions that produce primary products and dampen output in those that use primary products as an input. Because these activities are not uniformly distributed across regions in Canada, such shocks have implications for regional economic performance. A qualitative analysis of three episodes, 1974, 1978 and 1987, seems to broadly supportive of this theory. A statistical analysis using VARs also provides some support. Our results may have implications for other branches of the economic literature. One example is studies of long-term conver- 
gence between Canada's regions. Such studies might in fact be underestimating fundamental convergence because occasional termsof-trade shocks are tending to derail the process; alternatively, these studies might actually be detecting evidence of reequilibration to terms-of-trade shocks rather than fundamental convergence”.

\subsection{Trade-based Growth}

Although Canada's natural resource base (see the Staple Thesis) coupled with a small population and small internal market has forced it (despite the National Policy) always - to a greater or lesser degree - to look abroad for sales opportunities, since Confederation in1867 it could be said that the theme has been one a country focused on nation-building within and raw materials sales outside. Canadians have seen themselves as "exporters", but not really as either "traders" or "entrepreneurs". Indeed, many provinces over the years, relied on foreign entrepreneurs - in effect financed by provincial incentives of one kind or another - to use "mega-projects" to revitalize and restructure their economies. One of the most famous of these, for example, was a giant oil refinery at Come-by-Chance Newfoundland, developed under the auspices of an American businessman.

This passive/reactive mindset is, however, changing - witness the Alberta website materials cited above, and the initiatives described by the in-coming Prime Minister, Paul Martin below.

One model for this new re-orientation to "trade and entrepreneurship" is pre-Confederation Nova Scotia and New Brunswick (both now "have-not" provinces). Together, these two then colonies had the $4^{\text {th }}$ largest merchant marine in the world (source; RowellSirois Report). They built their own ships; they shipped their own produce (fish, farm goods); and traded with the US (New England for shoes and linens), the Caribbean (for sugar cane, molasses, and 
rum), and Britain (for linens and steel); and in doing so, earned both sale-of-goods profits, but also the shipping charges.

\subsection{Finance-based Growth}

As stated earlier, Canada has needed to rely heavily on foreign capital (with a heavy emphasis - first on British, then American sources) throughout its history. The Canadian Encyclopedia gives the following summary:

Foreign investment in Canada is very large, with the consequence that a considerable fraction of the economy is controlled by foreigners (mostly Americans). This large foreign presence in the economy, quite unparalled elsewhere in the world, has deep historic roots. Beginning in the mid- $19^{\text {th }}$ century, when Canada was still a British colony, British investors readily supplied capital, chiefly of the portfolio type, that financed construction of canals, railways, urban buildings and public works, in the half century prior to WW1. [And thereafter, there was heavy foreign investment in natural resources and branch plants.]

The Province of Ontario (Canada's largest with 41\% of GDP. Pop of some 12 million) Task Force on "Competitiveness, Productivity, and Economic Progress" issued a Report www.competeprosper.ca/task/ar2003.pdf November 25 ${ }^{\text {th }}, 2003$ highlighting a relative lack of growth in the Region (compared to a peer group of leading US States) and blamed a number of factors, working together to impair productivity, including:

a) lower investment by businesses in "machinery, equipment, and software, which are critical drivers of productivity and innovation”,

b) lower spending on education,

c) all levels of government (federal, provincial, municipal) shifting "their spending more towards areas that consume current pros- 
perity, such as health care and social services, and away from investment in education and infrastructure,

d) under investment in integrating immigrants "even though their qualifications and skills give us an advantage over our peer group",

e) under investment in "cities where the prosperity gap with the peer group is highest",

f) higher taxes - although the statutory corporate tax rate is lower in Ontario then the US, the US States allow bigger deductions for depreciation, charge lower capital taxes, and provide other tax breaks that reduce the marginal effective rate of taxation.

The point has already been made that Canada has always depended very heavily on investment from abroad. (Indeed, so did the US. In the $19^{\text {th }}$ century many US railroads were built with European, mostly British, money.) And, as above stated, there is currently more investment capital outflow than inflow.

That said, given that Canada has a national branch-banking system dominated by 5 big banks now largely centred in Toronto (Royal Bank of Canada, Bank of Montreal, CIBC, Bank of Nova Scotia, Toronto-Dominion Bank) there are two further points can usefully be highlighted here:

1) Australia (in its 1997 Wallis Report) has identified Regional Banks as important for regional development and for competition generally.

"Regional banks have been an increasingly important competitive force in recent years. In particular, along with credit unions and building societies, they have led the way on service, innovation and pricing on some products. Because consumers, including small businesses, are more likely to see regional banks, rather than credit unions and building societies, as acceptable substitutes to major banks, it is the former which have arguably imposed the greatest competitive pressure” (p. 455). 
While it can be said that Canada tried regional banks in the 1980s and that the experiment was unsuccessful (Canadian Commercial Bank and Northland Bank failed in 1985), it might rightly be said that the issue could bear re-examination.

2) Britain (in its 2000 Cruickshank Report) has noted that there is a lack of risk capital and bank loans are not at all a substitute.

"Fast growing small and medium sized enterprises have the potential to contribute to UK output and productivity growth. But to do so, they need access to external finance, since revenue growth often lags behind their investment demands. Achieving fast growth is a risky undertaking and many enterprises will fail in the attempt. So these SMEs primarily need access to risk capital at the key stages in their development. This chain of financing is only as strong as its weakest links. In the UK, the weaknesses appear to be:

- There is inadequate supply of early stage risk capital for start up and young companies, for whom traditional bank loans would be an inappropriate form of finance. This market failure in turn holds back the development of a group of enterprises which would then graduate onto larger scale venture capital;

- Public equity markets for smaller companies have been insufficiently vibrant to provide business angel and venture capital investors with timely liquidity when they wish to exit, which allows the proceeds to be recycled into earlier stage investments.

"Following on from this, the Government should examine all of its current and proposed policy interventions that are inappropriately focused on debt, such as bank finance for knowledge based businesses, with a view to redirecting the resources to equity support for SMEs" (pp. 178-9).

It could be argued that this analysis and recommendation should apply to Canada as well (perhaps more so) because of the dominant role in the financial system (including the capital markets) played here by the big banks. 


\section{RESEARCH-BASED}

Three early national research-based initiatives were establishing the Royal Society of Canada, the National Research Council, and the Experimental Farms.

In a nutshell - (i) the Experimental Farms developed Marquis wheat; (ii) Marquis wheat helped create the "Wheat Boom" on the Prairies; (iii) the "Wheat Boom" was the critical factor in opening up settlement and in making the National Policy work.

The Wheat Boom 1896 - 1913 (National Economic Expansion \& Integration)

"For twenty-five years [after 1867] the new nation [of Canada] had languished and even the most sanguine were troubled by forebodings about the success of Confederation. Then a fortuitous conjuncture of world circumstances brought with a rush the fulfillment of hopes long deferred. Life began to stir in the frame erected years earlier for a transcontinental economy. Directed by national policies of all-Canadian railways, western settlement and protective tariffs, it grew with a rapidity surpassing all expectations. A vast and sudden transformation was wrought by the magic of wheat.

The wheat boom brought a flood of settlers into the West and created two new and flourishing provinces [Saskatchewan and Alberta]. It precipitated a new era of railway development and spurred on the industrialization of Central Canada. Immense capital expenditures were necessary to equip the West and the growing urban and metropolitan areas of the East. Wheat worked a new integration of economic life and linked together the fortunes of the different regions ...

“The 'fortuitous combination of world circumstances' contained several elements particularly favourable to Canada: prices of raw materials rising more quickly than those of manufactured goods; the ready availability of capital at low interest; ... the phenomenal decline in ocean freight rates; and the relative cheapness of the 
costs of production in terms of selling prices. The Federal Government responded to these beneficent conditions by a more successful pursuit of national policies than had been possible in the previous years of world economic dislocations. With the end of the railroad land grants in 1896 the Federal Government made 60 million acres in the West available for free homesteads and along with the railway companies proceeded vigorously to attract settlers, so that by 1913 the arable lands in that region were almost totally occupied”.

The Development of Marquis Wheat

"It [Marquis Wheat] was first sown in a pure state in 1904 [at the Central Experimental Farm at Ottawa] ... [After baking tests in 1907 it was sent for trials in Saskatchewan.] its success in the prairie country was phenomenal. The year 1907 was quite unfavourable for most varieties owing to the prevalence of rust and of cool, wet weather. The early-ripening habit of Marquis and its power of resisting rust (to a certain extent) gave it an immense advantage ... Taking the average of the past five years (1907 - 1911 inclusive) Marquis has given 50 per cent more crop than Red Fife, on the uniform trial plots at Indian Head [Experimental farm]... In addition to its productiveness, the chief points in favour of Marquis, for the provinces of Saskatchewan, Manitoba and Alberta, are its earliness in ripening (generally from six to ten days earlier than Red Fife), strength of straw and comparative freedom from rust, heavy weight per bushel and fine appearance of the grain, and the excellent colour and baking strength of the flour produced from it”.

While the aim here is not to go into an analysis of "economic clusters", nevertheless, it is useful to highlight several points:

- Despite many of the opportunities of the "Global Village" (discussed later), many like and related industries tend to "cluster" together geographically.

- There is usually a university or research facility in that "cluster”. 
Ottawa, Canada's capital, for example, sees itself as the centre of at least 4 clusters:

a) Telecom - networking, transmission, satellite/microwave, communication interfaces.

b) Photonics - image sensing, multimedia, laser, light waves.

c) Health Tech - bio-tech, medical, health-related.

d) Microelectronics - component suppliers.

EDUCATION-BASED

One of the first reforms effected in Canada as it gained its independence bit-by-bit from Britain, was not only in many ways to adopt the Scottish model above, but to break the religious monopoly on schools and to insist on universal, free public education starting at the elementary level, then extending to secondary schools.

Despite a policy starting in the 1960s that made university education even more widely available than it had hitherto been, through grants and loans, years of austerity in university funding (most of which has in Canada come from government) have changed this. This austerity was generated by the need to curtail government deficits and address, among other things, the growing expenses associated with other pressing priorities, especially health care. Accordingly, two themes - both supposedly "markets" based have emerged:

(i) "user-pay" more along the American university model (for example, Law Schools in 1974 charged \$500 per year; now some charge $\$ 16,000$ to $\$ 20,000$ );

(ii) what universities "produce" should be more directly relevant to business.

SHIFT FROM AGRICULTURE TO INDUSTRY

At one point - especially during the Wheat Boom as earlier described - Canada was very heavily dependent on agriculture, with much of its population living on farms or in small towns. Technol- 
ogy, among other things, changed this. Canada's agricultural productivity is high, but it is driven by mechanization. And very few people now live in rural areas.

After the Second World War, in particular, Canada saw, with the rise of Industry: the rise of a well-paid, industrial (often unionized) workforce, the so-called "blue collar" middle class, usually hourlyrated, rather than salaried (as were the "white collar" office workers). But, recently the status quo has been shifting again: (a) with a rise is the "service" sector - including "knowledge workers" (discussed below); and (ii) with free-er trade and globalization, the growth of competing industry overseas. (Ross Perot in the US a few years ago called this a "Sucking Sound" of jobs going south to low-wage Mexico.)

The Post Second World War era in Canada also saw the "norm" as employment for life in a large organization (Alcan, CIL, Dupont, Abitibi, or a bank, a university, the army ...).

Suffice it to say that that "norm" no longer obtains for a growing number of Canadians.

SHIFT FROM INDUSTRY TO "KNOWLEDGE WORK"

Briefly, Peter Drucker has advanced the case that more and more work will be (in what used to be called the "service" sector) "knowledge work". And that necessarily places emphasis on how it is to be organized, and nurtured, and improved upon. More of this below (see the section on creativity and innovation in particular).

In short, this change of the centre of balance to knowledge work is happening, fast.

SHIFT FROM RURAL TO URBAN

Canada is not only now an urban, "cities-based" society, but it is concentrating in several major urban locations of in the order of at least 1 million each, especially: Vancouver, Edmonton, Calgary, Saskatoon, Winnipeg, Toronto, Ottawa, Montreal, Quebec City, Halifax. 
The general implication is clear - a new Cities-Based approach needs to be developed.

Canada's in-coming (on December $12^{\text {th }}$ ) Prime Minister, Paul Martin, has stressed this.

In his speech "Towards a New Deal for Cities" www.paulmartintimes.ca he stated:

"I want to confirm unequivocally that ... I remain firmly committed to forging a New Deal for Canadian municipalities, both large and small. Our cities are the engines of our nation's growth. Our cities are our signature to the world. It is clear that if Canada wishes to have a stronger voice on the global commons, then that voice will come from our cities, the places where the ideas of our nation coalesce around a thousand different ambitions - from sports to science, from manufacturing to music, from commerce to cuisine.

"It is clear that as the issues in Canada and around the world become more complex, the ingenuity to address them will come from places where people gather together in common cause.

"Indeed it is no coincidence that many of the world's most successful cities are home to one of the $20^{\text {th }}$ century's most productive developments: "Clusters," places where a concentration of talent creates a virtuous cycle of innovation and opportunity. Places where universities, companies, skilled labour, capital and governments converge to advance new breakthroughs ...

"in a world without walls - where the best and the brightest can live anywhere they want - it is evident that a country's success depends on the degree to which it becomes a magnet, attracting the best and brightest, whether home-grown or from beyond its shores.

"And the fact is that a country's strength as a magnet, is equal to the strength of its cities, [which in turn depend on] first, the ability to draw vastly different kinds of people together to dream and to innovate ...[And, secondly] the quality of life it provides ...” 


\subsection{Shifts from Region to Region}

A Bank of Canada "Technical Report" in 1989 on "Regional Disparities in Wage and Unemployment Rates in Canada" makes a number of relevant points respecting regional growth and movements and differences between regions:

"Simple multi-region economic models predict that in equilibrium, regional disparities in wage rates and unemployment rates will not exist. Yet this seems never to have been the case in Canada; the existence of regional income disparities since about 1900 has been well documented. The puzzle of why regional disparities exist and what to do about them has given rise to a large theoretical and empirical literature both in Canada and in other countries” (p. 1).

Under the heading "A survey of the causes of regional disparities" the Report surveys two types of theories - static and dynamic:

"The static models describe short- or long-run equilibriums in economies in which there is no growth. The dynamic models investigate the process of regional growth, with some models predicting the divergence of regional growth rates over time ...

"One shortcoming of the literature on the causes of regional disparities is that there seems to have been more emphasis on the development of theories than on testing them. In large part that may be because of a scarcity of data available at the regional level ... (p. 13).

"This [above] review of models from trade theory has identified a number of factors that can cause regional disparities in wage rates: increasing returns to scale, barriers to trade in goods, and barriers to the movement of capital and labour between regions. The evidence available suggests that these factors do exist and contribute to regional wage disparities ... (p. 16).

The Report then goes on to cite:

a) size of a region as aiding returns to scale,

b) internal barriers as distance and government policy (including regulation and subsidy), 
c) barriers to capital (including taxes and subsidies),

d) barriers to labour mobility (including moving costs, taxes, unemployment insurance).

With respect to Labour Mobility, the Report says:

"Most recent studies of migration in Canada have put particular emphasis on policy-induced barriers to labour mobility, such as regional differences in taxation, the provision of local public services and the availability of unemployment insurance benefits. Much attention has been focused on intergovernmental transfer payments from the federal to the provincial governments. Because such payments allow provincial governments to offer lower tax rates or higher levels of services than would otherwise be the case, outmigration from low-wage, high-unemployment provinces could be discouraged. The federally administered unemployment insurance program may have a similar effect because more generous benefits are available to individuals who live in high unemployment regions.” (p. 21).

\subsection{Focus on "Disparities"}

Tom Courchene (a leading Canadian political-economist) said the following:

"Equalization Payments are payments that the federal government makes to the poorer provinces. The monies come from Ottawa's [that is, the federal government's] general revenues and are unconditional transfers that can be spent as the recipient provinces please (see also transfer payment). Their purpose is to reduce the horizontal imbalance among the provinces.

"In general, 2 kinds of fiscal imbalance can arise in a federation vertical and horizontal. The former is an imbalance between the 2 levels of government, federal and provincial, eg, when the responsibilities of the provinces are disproportionately large compared with their share of revenues. Such an imbalance can be remedied 
by a transfer of responsibilities to the federal government (eg, family allowances and unemployment insurance) or by a transfer of revenues from Ottawa to the provinces. By contrast, horizontal imbalance is a fiscal imbalance among the provinces themselves - the fact that some provinces have more sources of revenue and are therefore richer then other provinces. Equalization payments can help adjust these horizontal imbalances.

"The Constitution Act of 1982 states 'Parliament and the Government of Canada are committed to the principle of making equalization payments to ensure that provincial governments have sufficient revenues to provide reasonably comparable levels of public services and reasonably comparable levels of taxation.' The concept of equalization can be traced to the statutory subsidies in the Constitution Act, 1867, and more recently to the National Adjustment Grants recommended by the Royal Commission on Dominion-Provincial Relations [Rowell Sirois Commission, 19371940] as part of an overall reorganization of federal-provincial financial arrangements ...

"The first formal equalization program was introduced in 1957. The transfers were designed to ensure that per-capita revenues of all provinces from shared taxes - personal income taxes, corporate income taxes and succession duties - matched those of the wealthiest provinces, at that time BC and Ontario.

"In the first of the required 5-year revisions, the level up to which these transfers were equalized became the all-province average ...

[In brief, the program continues with various permutations and complexities over the following years.]

At least to a degree, without more, these policies interfere with market allocation mechanisms, and thus can serve to impair possible further growth in the equalization giver and also the equalization receiver. 


\section{RELOCATIONS \& TRANSITIONS}

Both Canada and the US had a "Baby Boom" after the Second World War - demographically much larger than the groups immediately before or after. Thus as the "boom" matured, it generated higher than previous levels of demand for all kinds of products schooling, housing, jobs, health care, and - in the future - pensions.

As indicated: (i) Canada has needed immigration to grow; and (ii) with the Wheat Boom, there was extensive immigration to Canada from abroad, and considerable internal migration in response. Although much of the migration to the Prairies was both encouraged by the government and even subsidized, the allocations were fundamentally economic in nature, profit-motivated, if you will; and thus largely successful.

Without detailing them, suffice it to say that government policies (in Newfoundland and the Northwest Territories) encouraging people to move, so that government could provide services to them more effectively have been viewed as either unsuccessful or as having no better than "mixed" results.

\section{CREATIVITY \& INNOVATION}

For Canada, the coming together of technology, markets, and globalization with the new urban reality, and the need for creativity and innovation, at least, means several things:

- That "regional" focus will need to become more "city" centred.

- That education and life-long learning will need to receive renewed stress.

- That the system should be open to, and give opportunity to, all a diverse all.

- That SMEs will need more attention, and support, than they have hitherto received.

- That markets and competition will become more central.

It also means that - given the collapse of distance - that creativity and innovation, and the economic engine that they fuel can oc- 
cur wherever the right minds "cluster". No longer is there a need for over-aggregation either in remote natural resource-proximate locations, or in overly large urban "sprawls". The "best and the brightest" (to use the term from Paul Martin's speech) can, to a great degree, choose their location; and that choice will, more and more, be guided by "quality of life".

And, as well, it means the fostering of an attitude - an openness, a curiosity (a "thirsty mind"), an imagination, plus a practicality (since innovation is simply applied creativity).

ECONOMIC GROWTH AND PROVINCIAL DISPARITY (Extracts), Serge Coulombe (CD Howe Institute, March 1999)

Fiscal federalism has helped to remove many of the regional imbalances in per capita income among the provinces since the early 1950s, but given current institutional and political realities, the remaining disparities will persist, concludes a CD Howe Institute Commentary.

Coulombe notes that, despite Canada's huge size and geographic diversity, many disparities between rich and poor regions were gradually removed between 1950 and the mid-1980s through the "convergence phenomenon", whereby human and physical capital tend to accumulate more quickly in regions where they are relatively scarce. Interregional transfers, Coulombe argues, played their part by helping to finance improvements (in the form of better education and training) in human capital in the poorer regions, especially Atlantic Canada. This, in turn, helped to attract financial and physical capital into those regions.

Since the mid-1980s, however, the catch-up process generated by the convergence of capital seems to have exhausted its effects, and the disparities that persist reflect not only the industrial structure of the regions but also the institutional and political context. The downside of fiscal federalism, Coulombe argues, is that, by financing the delivery of health care, education, and income sup- 
port by the poorer provinces at levels comparable to those found in richer provinces, it encourages Canadians to remain in lowproductivity regions. Individuals who cannot find work in their home province need not, therefore, move to benefit from adequate public services.

In the future, Coulombe says, the regional distribution of Canada's economy will be affected by the relative decline of the manufacturing sector, the westward shift of the economic centre of gravity, and the continuing development of north-south patterns of trade in place of more traditional east-west patterns. These stresses could undermine Canadians' support for interregional redistribution. Yet, Coulombe argues, a transfer system more adequate than the current Canada Health and Social Transfer will be required in order to eradicate the under-funding of postsecondary education and improve the stock of human capital in the poorer provinces. Such an improvement would eventually make the poorer provinces richer by raising their level of economic.

Main Findings of the Commentary:

- Provincial disparities in per capita gross domestic product, per capita income, and productivity have lessened since World War II, but they are still substantially larger than those among US states.

- The persistence of regional disparity in Canada is the result of the country's diversity and its model of fiscal federalism.

- Since 1961, interprovincial migration has resulted in much redistribution of population, caused by the relative decline of manufacturing and the increase in farm productivity.

- Various economic indicators - per capita income, earned income, output, labor productivity, and the labor force participation rate - have grown faster in the poor provinces than in the rich ones since WW II. But disparities in unemployment rates have not lessened. 
- Disparities in human capital can largely explain the level and changes of provincial disparities in per capita income and output. Measuring human capital is difficult, but logical proxies such as measures of schooling, especially postsecondary schooling, suggest that the provincial distribution of human capital is indeed moving toward a national average.

- Federal government financing of university education in the poor regions can improve the Canadian social optimum. Without that funding, the poor provinces would tend to underinvest in postsecondary education. Since 1977, however, Ottawa's contribution to postsecondary education has been part of block grants not tied to identifiable spending in the provinces. That arrangement may be leading to underinvestment in education, especially in the poor provinces.

- Modelling suggests that the current level of provincial disparities has reached a steady state that reflects the industrial structure of the regions and the institutional and political context. Of course, regional economies will continue to be subject to unpredictable localized shocks, as with the effects of the failure of the Atlantic groundfishery.

- Nevertheless, disparities in Canada's per capita output are still a problem because of their size - about 50 percent higher than that of the disparities among US border states. This situation results from the fact that, because of Canadian policies on labor and employment insurance, Canadians are inclined to remain in areas of low productivity and high EI-benefit eligibility, even if they do not work. And they need not move to benefit from adequate public services.

The Commentary:

Canada's regional diversity has resulted in great disparities in provincial economies, but should this fact concern anyone except, 
perhaps, residents of the worst-off provinces ? [Yes] ... for at least four reasons:

[1] The first is the diversity of the country's settlement patterns ... The implication of this diversity is that Canada's various regions can follow different lines of development, that their economic cycles are not necessarily correlated, and that one industrial policy formula cannot be applied uniformly across the country.

[2] The second reason is the persistence of major economic disparities, which have been noted ever since Confederation. Although one can say that interprovincial disparities in per capita gross domestic product, per capita income, and productivity have tended to diminish since World War II - a phenomenon known as convergence - these differences are still substantially larger than those among US states. Their existence and persistence raise the problem of equity. They also greatly complicate the achievement of vertical and horizontal equilibrium in the finances of governments of a highly decentralized federation in which provincial and local administrations provide a large proportion of public services such as health care, education, and social security. The issue of substantial economic disparities is entangled with fiscal federalism. Indeed, Canada has traveled further down the road of interregional redistribution than almost any other federation (Courchene 1994).

This approach took shape mainly in the late 1950s and coincided with the birth of the welfare state. With the aim of promoting the introduction of national programs, Ottawa began to finance various provincial expenditures in health, postsecondary education, and social security. It set up an equalization program to ensure that provinces were able to offer public services of comparable quality without unduly increasing their tax burden, and the principle of equalization was entrenched in the 1982 Constitution [see 2 below]. Yet interregional redistribution is not an intrinsic characteristic of a federal system. In the United States, for example, the federal gov- 
ernment redistributes a smaller proportion of resources through transfers to municipalities and states, and this redistribution is not driven by the goal of equalizing fiscal capacity across local governments. Other federations, such as Germany, are in an intermediate position between the United States and Canada.

[3] Thus interregional redistribution is a third reason for concern about regional issues. High debt and interest rates [note; written early 1999] have tightened the efficiency constraint on public spending programs ...

[4] The last reason to be concerned about regional diversity is probably the most important. Study of Canada's regions is the best starting point for understanding the changing conditions and growth pattern of the country in general ...

EQUALIZATION \&THE CANADIAN CONSTITUTION: THE CONSTITUTION ACT, 1982

Part III - Equalization and Regional Disparities

36(1) [Commitment to promote equal opportunities]

Without altering the legislative authority of Parliament or of the provincial legislatures, or the rights of any of them with respect to the exercise of their legislative authority, Parliament and the legislatures, together with the government of Canada and the provincial governments, are committed to:

a) promoting equal opportunities for the well-being of Canadians;

b) furthering economic development to reduce disparity in opportunities; and

c) providing essential public services of reasonable quality to all Canadians.

36(2) [Commitment respecting public services]

Parliament and the government of Canada are committed to the principle of making equalization payments to ensure that provincial governments have sufficient resources to provide reasonably com- 
parable levels of public services at reasonably comparable levels of taxation.

IMPLY SHARING: AN INTERPROVINCIAL EQUALIZATION SCHEME FOR CANADA (Paul Boothe and Derek Hermanutz (CD Howe Institute, July 1999))

Canada's system of federal-provincial transfers is broken and needs fundamental reform, argues a CD Howe Institute Commentary that is critical of the existing equalization and Canada Health and Social transfer (CHST) programs.

In "Simply Sharing" [the authors] maintain that the existing equalization and CHST programs are seriously flawed. For example, the current scheme:

- transfers income from poor Canadians in rich provinces to rich Canadians in poor ptovinces;

- treats some provinces inequitably outside the formal equalization process;

- hinders economic development in less well off regions;

- blurs accountability, with Canadians not knowing which level of government to hold responsible for the taxes they pay and the programs they receive.

The authors argue that the existing transfer scheme is much larger than is necessary - currently more than two-thirds of the money raised to fund federal-provincial transfers ends up back in the province it came from. A smaller, more effective program would begin with Ottawa transferring income tax room equal to the total of federal-provincial transfer programs to the provinces. Provinces would allocate a portion of these revenues to a new interprovincial equalization fund. By design, initial contributions and withdrawals from the fund would ensure that the transfer of taxes left provincial budget balances unchanged, so that all governments would be no worse off at the outset. Future transfers among the provinces would be calculated using a simple equalization formula 
based on differences in provincial personal income, an arrangement that would avoid many of the existing scheme's problems.

Among the advantages of such a scheme, the authors say, are that equalization-receiving provinces would no longer face incentives that distort their economic development efforts. With transfers made directly between provinces rather than through Ottawa, voters would find it easier to relate the taxes they pay each level of government to the programs they receive. In addition, the federal budget would be insulated from swings in provincial fortunes that, through their impact on federal-provincial transfers, have affected Ottawa's bottom line in the past.

Main Findings of the Commentary

- Canada's current system of intergovernmental transfers is outdated and no longer serves the federation well.

- The current system has problems related to equity, efficiency, declining political viability, and sustainability.

- One result is that resources go from low-income families in some provinces to high-income families in other provinces.

- Equity problems for governments also occur: provinces receive different amounts of federal support for welfare, health care, and postsecondary education. In effect, these transfers are a separate equalization scheme, outside the formal program.

- The current system impedes efficiency-enhancing migration by individuals to provinces where employment opportunities are better.

- The existing scheme reduces incentives for equalizationreceiving provinces to stimulate certain kinds of economic development. It also encourages them to set tax rates higher than they otherwise would.

- The current system makes it difficult for taxpayers to know who pays for what. For example, in fiscal year 1996/97 about 
69 percent of the money Ottawa collected for transfers went back to the provinces from which it originated.

- Because equalization is paid by the federal government but is based on provincial revenues, large changes in those revenues can force major fluctuations in federal expenditures. For example, pressure on the equalization program was a major factor leading to the infamous National Energy Program.

- The recent federal-provincial review of equalization did little to address these problems. Neither the federal nor provincial governments used this opportunity to initiate an open, wideranging discussion on the future of this critical program.

- To that end, we suggest a fundamental reform of the transfer system. The proposed scheme would, first, transfer sufficient income tax points from Ottawa to the provinces to allow a set of net interprovincial equalization transfers. These transfers would be fiscally neutral for all governments, thus respecting the political bargain implicit in the current level and distribution of transfers. Next, the scheme would use a simple equalization formula based on a single macroeconomic indicator: adjusted personal income.

- Such a scheme could address many of the problems of the current system. Key benefits would include increases in transparency and the accountability of governments for the transfers they make and receive. The federal government would no longer be funder of the scheme, as it is currently, but guarantor of the new interprovincial find.

- Reforming intergovernmental transfers is worth the effort. By improving the incentives created by the transfer system as well as making it more transparent and accountable, Canadians can ensure that this important program will continue to support the federation in the future.

The Commentary: 
Commentators have long recognized that intergovernmental transfers, especially those under the equalization program, are a key element of Canadian federalism. Canada's system for sharing among the provinces is held to be one of the world's best examples of federalism at work.

Over the past decade, however, the intergovernmental redistribution system is waning. Concerns have been expressed that Ottawa's ad hoc changes to key transfer programs have increased the element of interprovincial redistribution in transfers originally aimed at funding programs for health, postsecondary education, and social assistance. In addition, the transfer system has become so complicated that it is virtually impossible for taxpayers to know how their tax dollars are being spent and which politicians to hold accountable. Some analysts also suspect that the design of certain intergovernmental transfer programs encourages inefficiency and impedes economic adjustment.

Equity - Equity concerns stem from the current transfer system's implications for the distribution of income among individuals. Academics have known for some time that the operation of transfer schemes that improve the distribution of income among governments can worsen its distribution among individuals.

Efficiency - Efficiency concerns about the current equalization program involve two questions: To what extent does it prevent efficient interprovincial migration by individuals? Does it distort the policy decisions of provincial governments?

Political Viability - Much of the recent decline in support for equalization has resulted from Ottawa's choosing to eliminate its deficit by reducing transfers to the provinces by much more than it has reduced spending on its own programs.

Transparency and Accountability - Issues related to the transparency and accountability of the system are also a concern. The 
current system is so complex that only a few experts in government and academe understand it.

Sustainability - To be sustainable over the longer term, intergovernmental transfers must be stable and reliable sources for recipients and affordable expenditures for contributors. The two characteristics are linked: transfer programs that prove unaffordable will, by their nature, also be unreliable.

\subsection{The Federal Spending Power: Scope and Limitations}

The concept of the federal "spending power" is a relatively recent constitutional development. It arises from federal government initiatives immediately following the Second World War, and is closely linked with efforts to centralize the taxing power. By providing program funds for a variety of health, education and social development programs, either unilaterally or in cooperation with the provinces, the federal government substantially altered Canada's approach to issues that were essentially within provincial jurisdiction.

The spending power thus became the main lever of federal influence in fields that are legislatively within provincial jurisdiction, such as health care, education, welfare, manpower training and regional development. By making financial contributions to specified provincial programs, the federal government could influence provincial policies and program standards.

Until the 1960s, most of the provinces acquiesced in this expanded federal influence, but Quebec both raised objections and refused to accept certain contributions. With the election of a new provincial government in 1960, Quebec's objections crystallized, and during the early 60s other provinces also began to find the increased federal role objectionable. Accordingly, in 1964 the provinces were given the right to "opt out" of programs financed 
through the spending power with income tax abatements as compensation. Only Quebec took advantage of this provision.

In June 1969, the federal government presented to a Federalprovincial First Ministers' Conference the paper "federal-provincial Grants and the Spending Power of Parliament”, which, for the first time, dealt with the evolving nature of the "spending power".

Ordinarily, one thinks of the "spending power" of governments simply in terms of the spending they do on particular programmes, under the authority of legislation passed by their legislative bodies. Constitutionally, however, the term "spending power" has come to have a specialized meaning in Canada: it means the power of Parliament to make payments to people or institutions or governments for purposes on which it [Parliament] does not necessarily have the power to legislate.

The federal paper noted that there was some disagreement among constitutional scholars as to the limits of the spending power. Some, such as Bora Laskin and GV LaForest [both later Supreme Court of Canada justices], argued that Parliament could make conditional or unconditional grants for any purpose, provided that the program did not amount to legislation or regulation within provincial jurisdiction. Others, including Quebec's Tremblay Commission, argued that Parliament had no power to make grants of any kind in areas within exclusive provincial jurisdiction. Yet others seemed to suggest that unconditional, but not conditional, grants could properly be made in areas within provincial jurisdiction.

The provincial governments had argued that the Government and Parliament of Canada ought not to be able to initiate cost-hared programs without obtaining a provincial consensus, because the operation of such programs fell to the provinces; that cost-shared programs forced the provinces to alter their spending and taxing 
priorities; and that the citizens of provinces that "opted out" were subjected to "taxation without benefit".

The federal government, on the other hand, stressed the importance of the spending power in maintaining equal opportunity for individual Canadians (eg family allowances); in equalizing provincial public services (eg health, welfare, education and roads); in regional economic development; and in carrying out programs of national importance, such as Expo '67 [Montreal World's Fair in 1967].

In the result, the federal government "tentatively advanced" certain principles: (1) the federal spending power should be entrenched in the Constitution; (2) Parliament should have an unrestricted power to make unconditional grants to provincial governments for the purpose of supporting their programs and public services; and (3) Parliament's power to initiate cost-shared programs involved conditional grants in areas within provincial jurisdiction should require both a broad national consensus and per capita reimbursement of the people (not the government) of a province whose legislature decided not to participate.

The debate over the spending power continued on a muted but steady level through various constitutional negotiations in the 1970s and the 1980s.

In 1986, limitations on the federal spending power became one of Quebec's five conditions for support of the Constitution Act 1982.

As a result, the Meech Lake Accord of 1987 would have added a new section, section 106A, to the Constitution, immediately after the federal power to appropriate funds. Section 106A would have provided for reasonable compensation to the government of any province that chose not to participate in a cost-shared program in an area of exclusive provincial jurisdiction, provided the province 
carried on a program compatible with national objectives. [However, the Meech Lake Accord was not passed.]

\subsection{A New Approach to Regional Development in Atlantic Canada}

In This Issue :

Critics have long charged that federal regional development subsidies for Atlantic Canada are poorly targeted, ineffective and prone to political influence. This Commentary evaluates the case for regional development reform and argues that business grants should be replaced by measures to reduce existing tax burdens on business investment.

The Study in Brief:

This Commentary makes the case for a fundamental reform in the delivery of federal development assistance to Atlantic Canada. It argues that the federal government should replace existing grants and tax credits to businesses with a broad-based reduction of corporate taxes in the region.

Existing grant programs are well intentioned, though poorly targeted. Governments are usually not good at picking winners - but losers tend to be very good at picking governments. As well, grants may serve political, rather than economic objectives. This paper contains a quantitative analysis of the allocation of federal grants in Atlantic Canada in the 1988-to-200 period, providing evidence that supports this contention.

Since direct grant programs are ineffective, we recommend federal business tax reductions for the region.

A tax cut for Atlantic Canada could be implemented in a variety of ways; we consider two of them. A federal corporate income-tax rate cut of 6.5 percentage points on Atlantic income could be provided to replace about $\$ 250$ million of existing grants. Alternatively, incentives for capital investments could be provided to en- 
courage new activities in the Atlantic. This Commentary argues that a broad-based tax credit at a 10.5-percent rate should replace cash grants, as well as the existing federal Atlantic Investment Tax Credit, which is seriously flawed in design. Under this proposal, we estimate that the effective tax rate on marginal investment projects in the Atlantic region would be almost eliminated for many investment projects.

The Commentary:

Since 1988, the federal government has transferred nearly $\$ 4$ billion to businesses, governments and non-governmental organizations in the Atlantic provinces through the Atlantic Canada Opportunities Agency (ACOA). Recently, a number of critics have called for a re-examination of the agency's mandate and a fundamental reform in the way that regional development assistance is delivered, including substituting corporate tax cuts for Atlantic businesses for ACOA grants. This Commentary evaluates the case for regional development reform. It argues that Ottawa should replace business grants with tax measures directed towards investment, while revamping federal Atlantic investment-tax credit that has been poorly structured in the past.

ACOA is just one of a handful of federal agencies that direct subsidies to businesses in all regions of the country. We focus on ACOA because its expenditures in per capita terms for the Atlantic region exceed those made by other agencies and the federal government in other parts of Canada. At the same time, we do not question the important role of the federal government in contributing to public services in have-not regions using, for example, the Equalization program. [underlining added]. Rather, our analysis is directed more narrowly at direct subsidies to local businesses for the purposes of regional development. Certainly, some of our analysis could equally apply to other regional development programs. 
Regional development grant programs exist in many countries. Their aim is typically to help fledgling businesses obtain financing that private lenders do not provide - the idea being that many lenders are insufficiently knowledgeable about profitable opportunities for investment or are unwilling to take on the risks. A further aim of regional development programs is to help regional economies "get over the hump", to use the vernacular - to establish sufficient investment in productive capital and infrastructure to enable a region to become a magnet for other businesses and for a skilled work force.

While the road to development in Atlantic Canada has long been paved with such good intentions, poor design and implementation of policies has created some significant potholes. Several deficiencies can arise that undermine the usefulness of regional development grants... Grants often appear to be directed to infra-marginal investments - projects which would have been undertaken even in the absence of government support. As well, the grants create more demand for land and capital components, resulting in higher rents and capital goods prices, without generating the new activity that was originally intended. Even when grants spur truly new investment, little evidence can be found to support the conclusion that such projects can be made sustainable and profitable in the long run. At worst, inefficient businesses favoured with grants drive out competing profitable businesses that are not given the same assistance. Because of the cost of raising taxes to fund grants, the lack of benefits would be a very serious concern to taxpayers who have to ultimately fund the activities.

Because the Atlantic provinces have been catching up to Canada in per capita income in the past two decades, it can be argued that the regional development programs have helped improve the economy. On the other hand, the Atlantic region may be showing vigorous growth through its own entrepreneurship - as well as benefiting 
from significant investment in energy - with regional development programs reducing, rather than improving, productivity by keeping inefficient businesses in operation.

Loser's Choice - To some extent, these failures reflect the inevitable problems facing any government agency that lacks sufficient information to target its finds at areas where they can be most useful. Governments are unlikely to be very good at picking winners but losers tend to be very good at picking governments. However, targeting failures may also reflect other non-economic objectives of governments: cash transfer programs like ACOA may be used to reward political supporters, to buy votes in swing ridings, and for a variety of other political objectives, as well as for their legitimate role as a tool of regional industrial policy. Political scandals have dogged ACOA and the other federal and provincial regional development agencies on a number of occasions in the past. Below, we present some preliminary evidence on how political considerations may have influenced the allocation of ACOA funds.

If, as we argue, direct grant programs do not work well, then what is the alternative? Some critics have proposed eliminating federal regional development programs entirely, which would be a radical departure from past practice that we do not evaluate or take a position on here. Instead, we focus on the narrower question of how the federal government might best deliver a specified level of support to the Atlantic region. A number of Atlantic politicians have raised the possibility of replacing ACOA spending with a cut in federal business taxes of equal value for the region. Replacing direct grant and loan programs with tax cuts would eliminate some of the problems in targeting development assistance, and we believe the alternative deserves serious consideration.

What Does ACOA Do Anyway? - ACOA was established in 1987 with a broad mandate to "increase opportunity for economic development in Atlantic Canada and ... enhance the growth of 
earned incomes and employment opportunities". The agency interprets this mandate liberally, offering loans and "non-repayable contributions" to a wide variety of businesses, non-governmental organizations, and provincial and local governments for a wide variety of purposes. Recipients must apply to the agency for funds and eligibility is determined on a case-by-case basis according to a set of criteria that include incrementality, economic viability and related considerations ...

What's Right and What's Wrong With ACOA? - The core of ACOA's strategy for the Atlantic region has been to award company-specific investment subsidies, either in the form of outright grants or repayable contributions. Proponents argue that subsidies often play a useful role in encouraging investment, transferring technologies, developing markets and ultimately, raising wages and increasing standards of living in the region. Critics point to informational and political failures in delivering the subsidies and the inefficiency that may result. Here we offer a brief review of the case for and against ACOA.

Investment Subsidies and Capital Market Failures:

The economic argument for government intervention in the regional development arena, as in other areas, is that government policy may succeed where banks, entrepreneurs and other private organizations do not. In this case, the usual story is one of capitalmarket failures. Fledgling businesses may find it difficult to obtain private financing if banks worry about default risk and local sources of finance are undeveloped. If investment subsidies are to be an effective use of scarce government resources, however, then they must be targeted at investment projects that would not be undertaken in the absence of such assistance. However, there are many reasons to believe that a significant fraction of projects financed through ACOA is not incremental in this sense ... 
The actual success of ACOA in this regard [incrementality] is difficult to gauge because there is little real cost-benefit analysis of the grants. ACOA itself estimates that 84 percent of jobs funded under its flagship Business development program are incremental.

But that figure is calculated by comparing employment growth of assisted companies to a sample of other companies in the region. Since ACOA's aim is to finance economically viable enterprises, however, it is not surprising that assisted companies grow faster than average, and the approach tells us little about what would have happened to these companies in the absence of grants. A full study would have to use a methodology to account for the potential displacement of resources to subsidized from unsubsidized sectors of an economy. ACOA also provides estimates of the net impact of its activities on Atlantic GDP, using a macroeconomic simulation model. But evidence from other sources indicates that the number of new jobs created is relatively small, and that the cost per job created are high. One government study examined the Cape Breton Investment-Tax Credit, a federal program established in 1985. While this program was delivered as a tax measure rather than a spending program, companies were required to apply for assistance and eligibility was at the discretion of program administrators. In other words, CBITC operated in a way that was quite comparable to ACOA's company-specific grants. The government study estimated that only about 20 percent of investment projects funded by CBITC were truly incremental. As a result, the cost of the program was quite high relative to ostensible benefits - the foregone tax revenues under the program were equivalent to an estimated onetime payment of $\$ 700,000$ per job created.

Even if funding is confined to projects that are truly incremental in this sense, they may still have undesirable economic effects. These include: 
- Bad projects that receive support may drive good ones out of the market.

- Inefficiencies in local production techniques.

- Prices of land and capital goods might rise.

- Administrative overhead... and deadweight costs.

Some countries, particularly the East Asian Tigers like Singapore and Korea, have used industrial policy to expand successfully in the past, and have done so by targeting particular sectors and companies. B ut these have been very much the exception to the rule, and even in those cases, governments usually delivered assistance through the tax system, rather than through direct spending programs. Since the Asian financial crisis of 1997, some doubts have arisen about the success that the Tigers have really had in using targeted tax cuts or subsidies to achieve economic development.

\subsection{Infrastructure Grants and Agglomeration Economies}

A special challenge for development in have-not regions is that they lack what is known as agglomerations of skilled labour and capital, and the access to consumer markets that help make new investment profitable. An old justification for government industrial policies, lately somewhat resurgent, is that they can help underdeveloped regions to exploit a virtuous cycle of growth, in which initial strategic investments create an environment in which investment is more profitable for all. Rosenthal and Strange (forthcoming) provide a good survey of current research on the role and extent of such agglomeration economies in urban growth. The initial evidence is intriguing and contains some lessons for the role of industrial policy.

For one thing, if urban economies of scale are an important part of the growth phenomenon, then even greater agglomeration of economic activity is an inevitable consequence of growth- 
enhancing policies ... That is, the best growth-enhancing policies will probably also raise inequality within the Atlantic region, and will involve more, not less, movement of labour from rural to urban areas within the region. Viewed in this light, ACOA's propensity to direct much of its assistance to the poorest and least industrialized parts of the region must be seen not as part of a growth strategy for the region, but rather as a very inefficient kind of social policy.

For another, the existence of agglomeration economies indicates that certain investments in public infrastructure, particularly transportation, may play an important role in spurring growth. Infrastructure spending has been part of the mantra of Liberal politicians in Canada since the Red Book promises of 1993, and it is now a big part of the mandate of ACOA, too. Initially the federal government's emphasis on infrastructure received much intellectual support from research showing that public capital had strong positive effects on growth in local economies. But, as Crowley (20000 has argued, much of what is delivered [under] the rubric of federal infrastructure programs - in Atlantic Canada and elsewhere - is really about expenditures for current consumption and community development, and not at all about investment in productive public capital.

The Politics of Regional Development

Inevitably, the way in which subsidy programs are allocated among companies and localities in the eligible regions is at the discretion of officials, and decisions on allocation are rarely transparent. This raises the spectre that funding decisions reflect the political calculus of the government of the day as much as legitimate economic development objectives ...

Grants vs. Tax Cuts

If discretionary spending programs are a bad way to deliver regional development assistance, then what is the alternative? Some 
commentators have suggested that direct spending programs be replaced by tax reductions for companies investing in the region. Admittedly, tax measures have costs as well as benefits when compared to direct spending. The case for tax cuts in place of grant programs depends, in part, on the type of tax cut being considered targeted or broad-based.

Some tax cuts, such as investment-tax credits, tax holidays, flow-through shares or tax-free zones, can be targeted to specific companies, activities, and types of investment. Consequently, they may share some of the economic costs arising in grant programs. Like grants, targeted tax cuts are chosen by governments trying to pick winners or gain votes. Instead, targeted cuts possibly result in the subsidization of uneconomic activity that replaces unsubsidized profitable activity. They may also lead to higher asset prices, without spurring new activity and leave less revenue available to fund basic public expenditures like education and health.

Broad-based tax cuts are not aimed at particular activities and therefore benefit the economy more generally. However, as an instrument to achieve certain aims, such as building infrastructure or supporting education, the broad-based tax cut is unable to achieve certain aims without costing significant revenue.

\section{Grants vs. Targeted Tax Cuts}

Grant programs provide certain advantages over targeted tax cuts for three reasons: accountability, broader application, and cost control.

- Grant programs are scrutinized by Parliament.

- Grants support projects regardless of the taxpaying status of the company.

- The program costs are fixed. 
The case for targeted tax cuts is argued on other grounds, as well. For one thing, tax relief benefits the successful enterprises because only profitable companies pay taxes ...

However, the open-endedness of tax-cut programs leads to a different concern. When governments provide tax assistance to fledgling companies to offset capital-market failures, the resulting new entry into the market can undermine the profitability of existing, high quality businesses.

\section{Grants vs Broad-Based Tax Cuts}

Cogent arguments can be made both for and against grants or targeted tax cuts. But a better alternative than either, we argue, is to replace ACOA with a broad-based corporate tax cut in the Atlantic region.

Universal tax cuts are appealing for several reasons. The cut applies more generally and neutrally so that many taxpayers are given incentives to work, make investments and heighten productivity. Supporting arguments are based on the principle that the tax system is most efficient and fair if businesses bear similar tax burdens ...

Recent experience in Ireland where the government cut corporate taxes for manufacturing and financial-services income in the 1980s, and more generally for all businesses in the 1990s, indicates that broad-based tax cuts could be critical to improving per capita incomes.

Looking for a Better Way - In our search.

In our search for a better approach to regional economic development, we have come to the conclusion that a more neutral, broad-based tax cut would achieve more balanced growth in the Atlantic, compared with targeted funding of certain virtuous businesses selected by the government of the day. Such a reform could be implemented two ways - as a general cut in federal statutory tax 
rates applied to Atlantic income, or as a broad-based incentive for capital investments in the region.

REVITALIZING THE MARITIMES (National Post Newspaper, June 4, 2004 - Don McIver, Halifax, Nova Scotia)

The need to revitalize Canada's underperforming Maritime economies is garnering little attention during this [federal] election campaign. Thank God. Far better the focus be on the state of health care, the tax-spending balance and even the needs of municipalities.

For decades, federal politicians have shown up at election-time, declaring they are 'here to help', thus presaging a disastrous policy outcome. Their well-meaning efforts, with promises to close the prosperity gap with the rest of the country, have retarded the Maritimes' economic convergence with the rest of Canada.

A few weeks ago, the Atlantic Institute for Market Studies released a comprehensive study [see next heading below] detailing the perversity of such programs as Employment insurance, which subsidizes seasonal work, discourages education and causes labour shortages; an equalization formula that discourages local initiatives and distorts taxes; and the regional development arm of Ottawa, the Atlantic Canada Opportunities Agency (ACOA), through which the taxes of successful businesses are used to subsidize the unsuccessful.

For too long, 'economic development policy' has been based on the government-knows-best model - a concept that presumes mandarins, rather than entrepreneurs and investors, are the best judge of which business opportunities should be capitalized.

The argument for government intervention is based on the erroneous presumption that markets just aren't working in this region. Government planners believe that investors are somehow systematically ignoring profit-making opportunities - opportunities that planners funded by Ottawa can identify and nurture. 
This vision of how to achieve growth and convergence is dead wrong. It is contradicted by international experience and by Atlantic Canada's own history.

In the postwar era up to 1971, Atlantic Canada's per capita economic growth was strong, consistently outpacing the rest of the nation. Then, when Ottawa dramatically increased regional subsidies in the early 1970s, the region's growth began to falter. The reason is clear: Bureaucrats have neither the insight nor the experience to choose winners more effectively than the marketplace, and the track record of grants and loans targeted at specific projects is decidedly spotty.

Bricklin Motors is one of the most famous examples. Lured by millions in loans guaranteed by the province of New Brunswick, an American sports car producer opened up shop in the province in the 1970s only to go bankrupt, leaving taxpayers to foot the bill.

Another example can be found in Nova Scotia's Sydney Steel Corporation, which received \$275-million in government financing in 1987 to no avail. The company's plant fell into the hands of the Nova scotia government, which eventually had to sell it at great cost to taxpayers.

Adding insult to injury, there is a real risk that publicly subsidized operations drive competing firms created with private capital out of business.

More unsettling is the use of ACOA to serve political objectives. When it was created, ACOA was intended to be the lead agency in the region - the 'local face' of the federal government so-to-speak. Having Ottawa pour tons of money into the region, during the lead-up to an election or at the time of a potentially unpopular policy decision, could be advantageous for the incumbent government, no matter what its political stripe. More particularly, it could be a boon for federal politicians either elected by a slim majority or 
those elected in response to a previous government's mishandling of key regional policies.

There are concrete alternatives to ACOA. Lowering tax rates for all businesses, rather than relying on stimulative government spending, would remove the politics from regional development activity. Profitable businesses could then keep a larger share of their revenues, automatically rewarding successful enterprises and encouraging them to become even more successful. Government would no longer need to - or be able to - choose winners.

Regionally differentiated federal tax rates or possibly an Atlantic investment tax credit could achieve this goal. Alternatively, the Atlantic provinces could achieve additional fiscal flexibility if [the] federal government absorbed some provincial debt. The important objective is to develop a broad, tax-driven means of encouraging business development in Atlantic Canada - not one that targets particular industries or firms. That will help bring Atlantic Canada back into the economic mainstream.

\subsection{Prospects of the Development of Atlantic Canada}

How Ottawa Can Put Atlantic Canada on the Road to Prosperity (Atlantic Institute for Market Studies, Halifax, April 2004 - Crowley \& McIver)

Misguided federal policies not only have failed to the economic discrepancy between the Atlantic provinces and the rest of Canada; they have, in fact, held back the natural process of convergence, which could have closed the gap relatively quickly. Ottawa's policies of regional development spending, equalization transfers, and regionally extended employment insurance benefits are wellintentioned failures.

They have left Atlantic Canada with per capita gross domestic product that is no more than three-quarters of the national average, well below average productivity levels, and unemployment that is 
high even as the region suffers from increasingly significant labour shortages., Moreover, excessive federal regulation and shortsighted bureaucratic interference have prevented key industries in the region, such as the fishery and offshore energy, from acting as catalysts of economic revitalization ...

Canada's political leaders must recognize that improving Atlantic Canada's economy will depend on reversing ill-considered measures and introducing effective new policies ...

At a minimum, a new federal government that wishes to put Atlantic Canada on a trajectory toward growth and prosperity should take the following steps:

1. Dismantle expensive, politicized, and distortionary regional development programs by

- using broad, tax-based measures to foster a development-friendly business environment, and scrapping inefficient, politicized, project-specific incentives provided through agencies such as the Atlantic canada Opportunities Agnecy; and

- replacing programs aimed at individual businesses with federally driven corporate tax reductions that allow consumers to choose which businesses will prosper.

2. Revise the equalization program to reduce its perverse incentives, by

- re-establishing a ceiling on the total amount available for equalization;

- removing natural resources from the equalization formula; and

- swapping equalization payments for a provincial debtreduction plan.

3. Restore employment insurance (EI) to its original objective of protecting workers from unpredictable short-term interruptions in life-long attachment to the workforce, by 
- ratcheting down EI benefits significantly every year for annual repeat claimants;

- requiring first-time claimants to have at least one year of continuous work to qualify for EI benefits;

- making EI experience rated, so that employers pay higher premiums when they repeatedly lay off people who then claim EI;

- funneling a significant part of the savings generated by the EI reforms into vouchers to allow workers to gain access to a wide range of job skills and training; and

- eliminating regionally extended EI benefits.

4. Develop a business climate and regulatory structure that promotes strong growth in industries, such as the fishery and offshore energy, where the region has comparative advantage, by

Case Study \# 1: The Fishery and Aquaculture

- transferring ownership and control of the fishery to those who make their living from the resource, and making fish quotas fully transferable and tradable;

- passing a National Aquaculture Act that creates strong property rights in the ocean resources required for the aquaculture industry to expand and thrive; and

- transferring jurisdiction over aquaculture from the Department of Fisheries and Oceans [federal] to an 'industry-oriented' department such as Agriculture or Industry [federal];

Case Study \# 2: Offshore Energy

- creating the conditions in which an internationally competitive oil and gas exploration and development industry can take root and grow by keeping regulatory and tax costs competitive with those in other jurisdictions;

- streamlining the regulatory process for oil and gas to match approval times in competitor regions, such as the North Sea, that are suitable benchmarks; 
- eliminating jurisdictional overlap and coordinating policies to move toward a 'one-window' solution for the oil and gas industry; and

- introducing 'performance-based' regulation.

5. Build a new, strong, and committed relationship with the US, Canada's most important international partner, that reflects the interests of Atlantic Canada as well as those of the country as a whole, by

- supporting Washington's study of the transportation infrastructure in the northeastern part of the continent, stretching from Halifax to northern New York state;

- working with the US to create the needed infrastructure that ties Atlantic Canada more effectively into markets in the NAFTA heartland; and

- building on the Smart Commerce initiative to accelerate and further simplify border-crossing procedures, and working with the US on integrated perimeter security, the harmonization of external tariffs, and mutually agreeable standards of entry for persons from third countries.

Introduction: Converging on the Future

For decades, federal government policies have tried without success to narrow economic discrepancies between Atlantic Canada and the rest of the country - indeed, such policies have actually retarded economic convergence. Take unemployment, for example. Studies published by the Atlantic Institute for market Studies (AIMS) have shown ... that, although unemployment rates in Atlantic Canada were similar to the national rate until the end of the 1960s, with the liberalization of unemployment insurance rules in 1971 and the introduction of massive economic development policy spending in the 1970s and 1980s, unemployment and growth rates in Atlantic Canada and the country as a whole began to diverge. 
Half a century of economic theory suggests, and real-world experience - in places as diverse as Ireland, Japan, the Netherlands, South Korea, and US states such as Georgia and Michigan - confirms, that lagging economies naturally catch up with advanced ones. Even if advanced nations or regions grow quickly, lagging ones should grow even more quickly.

Under certain conditions, a lagging region should close the gap in economic performance with its relevant leading economy by 2 to 3 percent annually ... Yet Atlantic Canada's economy has converged with that of the rest of Canada at less than half that rate despite - or, more likely, because of - massive federal intervention.

Lagging economies that are closing the gap with advanced economies share a number of characteristics:

- an educated populace or, at a minimum, an emphasis on raising the educational standard of the population;

- a market economy and limited government interference in markets;

- the rule of law;

- property rights; and

- stable institutions, including political stability.

Ottawa's policies, however, have actually undermined some of the necessary conditions for convergence, by encouraging development by bureaucrats rather than by sound business planning, by regulating without full regard to long-term benefits, and by failing to develop clear systems of property rights in resources such as the fishery.

In the right setting, convergence occurs as a result of the spread of productive ideas and methods, the creation of profitable opportunities in economies with chronic underinvestment, the paying of competitive wages that draw capital, and the development of an increasingly skilled labour force as investment creates jobs. 
Of particular relevance are the different ways in which labour and capital combine and fructify in different kinds of economies. When labour is abundant relative to capital, labour costs should be relatively low and potential returns (profits) on the scarcer resource (capital) should be relatively high. The profit motive attracts capital, which creates jobs and economic growth. This mechanism, however, can be derailed by policies that either inflate the cost of labour or reduce returns on capital.

For a practical example of convergence, take Ireland, whose economy, until just a few years ago, was among the walking dead of Eurpoe. Now, it is one of the most successful in the developed world. This turnaround did not come about accidentally or because of fortuitous resource discoveries, but because of consistent, deep, and widespread policy changes that reformed Ireland's economy from top to bottom.

Ireland's economic purgatory, like its subsequent remarkable recovery, was policy induced. Getting the policy framework right made the difference between productivity and prosperity on the one hand, and unemployment and decline on the other. The foreward to an AIMS study summed up the right policy in the following way:

[The Irish] saw that trying to prop up dying industries was a mug's game. Public debt needed to be brought under control, taxes lowered, and excellent value offered in public services when measured against the taxes paid. Politics needed to be banished from decisions about where and how to invest, whether in public infrastructure or private industry. Work incentives needed to be improved by reforming social welfare. Profitability in the private secotr needed to be improved. And costs, including labour costs, needed to be kept keenly competitive. The sum of these measures was a policy environment in which business had every reason to invest and build productive capacity, while workers had every reason to work hard and build their job skills. As the capital invest- 
ment grew and workers became more skillful, real wages rose along with tax revenues, and a virtuous circle was created. Growth bred more growth, success bred more success.

Ireland's turnaround did not happen because of governemtn spending, transfers from the European Union, or an activist 'economic development policy'. Rather, Ireland, like other jurisdictions that have harnessed the forces of convergence, focused on getting the policy right and then letting employers and workers respond to the signals of the marketplace.

In Atlantic Canada, however, much 'economic development policy; has been based on the government-driven model, which largely explains why, despite heroic efforts by the federal government to encourage economic growth, the region has failed to converge strongly with Canada's 'have' provinces. In the postwar era up to 1971, Atlantic Canada's per capita economic growth was strong, consistently outpacing the rest of the nation. Only when Ottawa dramatically increased regional subsidies in the early to mid-1970s did the region's growth falter relative to the rest of Canada.

Programs such as employment insurance, equalization, and regional development initiatives aimed at accelerating convergence and minimizing the economic disparity gap have instead encouraged Atlantic Canada to rely on Ottawa's generosity and have obstructed long-term skills training and enhancement. Former New Brunswick premier Frank McKenna, in his 1997 farewell speech, observed that dependency has become a narcotic to which Atlantic Canadians have become addicted.

The evidence demonstrates that success for Atlantic canada can come only from policies that focus sharply on productivity, investment, competitiveness, appropriate social support, removing disincentives within equalization, and eliminating barriers to the free functioning of the labour market, so that the region's unemployment can be absorbed and labour shortages eliminated. 


\section{Atlantica: The International Northeast}

Atlantic Canada is part of a region that AIMS' authors call 'Atlantica', which broadly encompasses the Atlantic provinces, eastern Quebec, the northern tier of the new England states, and northern new York state.

Geography has placed Atlantica near the centre of the two largest trading relationships in the world. On the one side is the Canada-US trading relationship, the largest in the world at $\$ 2$ billion per day; on the other is the trading relationship between North America and the European Union which accounts for 40 percent of total world trade.

Atlantica's relative degree of underdevelopment can be explained in large part by the failure of policy makers to think of the region as a whole, where local success depends on working effectively across boundaries to achieve the economies of scale, transportation efficiencies, and other regional coherences that more successful regions - such as the US Midwest and Ontario or Texas and Mexico - take for granted. In short, Atlantica's political and natural disadvantages have been exacerbated by the Canadian and US governments' relative disregard for the region's economic requirements. On the Canadian side, the cumulative effects of more than a century of policies favouring the population centres of Quebec and Ontario are crumbling infrastructure and provincial governments and electorates corrupted by large transfer payments. On the US side, the northern New England states have been the losers in political battles with richer, more powerful states such as California, Texas, New York and Massachusetts.

Free trade and globalization now give Atlantica an opportunity to establish its rightful place in the continental economy. If the border cannot be made to disappear, its impact must at least be blurred. The introduction of vehicle-handling efficiencies and the building of new crossings at the border will help, but the concept is much 
bigger than that. Ideally, commercial relationships should be equally attractive across provincial-state lines between the two countries as they are across political boundaries within the two countries. Canada's Atlantic provinces and the US northeast must become as economically integrated and coherent as is, for example, southwestern Ontario and the US Midwest. Only then will Atlantica realize its economic potential.

In an election campaign that has centred on the Liberal [federal] government's cavalier handling of public money, you might think a guy who left Cabinet amid accusations of nepotism would be a little sheepish. Not Lawrence MacAulay.

Seeking his fifth mandate as the MP for Cardigan in eastern PEI, the former Solicitor-General continues to deny any wrong-doing and is asking voters to judge him on his impressive record of bringing federal dollars to the riding.

"I thought when I was elected here, it was my job to make sure that this district received its fair share", he said yesterday as he took a break from campaigning. "The truth is, we got some. It's quite a thing. I would never in my wildest dreams have thought that I could have been criticized for taking too much to my district.” ...

It is true that people assess their politicians differently on the tight-knit Island, where patronage is so embedded that snow-plow operators and road construction crews can lose their jobs for supporting the wrong party.

Mr. MacAulay's newspaper advertising has hammered home the message that he has delivered the goods for PEI since being elected in 1988, boasting that the Liberal government spent \$70-million on job creation in the riding.

Kendall Docherty, manager of Royalty Hardwoods Ltd., saw some of that money when ACOA, the federal government's regional development arm in Atlantic Canada, helped fund an expansion three years ago. The subsidy helped increase the company's. 
Workforce to 35 from five, he said. "I believe PEI as a whole will support the Liberals because they've done a good job for us. They've certainly helped the Island", he said. ...

The notion that political considerations influence Ottawa's regional-development in Atlantic Canada is more than folk wisdom, according to new research by economists Kevin Milligan of the University of British Columbia and Michael Smart of the University of Toronto.

Their analysis of ACOA grants from 1998 to 2000 found that ridings represented by a minister received more funding per capita, as did ridings where the previous election was closely contested. The economists theorize that governments focus on such swing ridings because they can get more bang for their buck.

"If we decide as a society that we want regional development programs, I think it would be best to have a way of doing that that doesn't favour one set of people over another on an arbitrary basis”, Mr. Milligan said in an interview. 


\section{Main Conclusions and Economic Policy Recommendations}

The research findings regarding convergence processes in Russia's regions and decomposition of their growth allow conclusions and recommendations as follows:

1. In the period in question (1994-2002), the average (across all the regions) Gross Regional Product per capita level (in constant prices) has been growing, albeit unevenly. A steady growth of the index was noted since 1999.

2. Since 1999 the growth in the average level of GRP per capita has been accompanied by a growth in the median value of per capita GRP. This means that the growth in the level became possible thanks both to a further rise in the welfare of the most prosperous regions (for instance, Moscow and oil producing regions) and the increase in per capita income in poor regions (whose income levels initially had been below the average level).

3. The dispersion of regions in terms of per capita income (in constant prices) has remained unimodal for each year of the period in question, i.e. there exists the trend to concentration of values of GRP per capita around the average (growing) value. According to Quah, this forms a necessary prerequisite for matching the hypothesis of convergence.

4. However, the analysis of the annual dynamics of dispersion of GRP per capita does not allow to argue that the differentiation between regions in terms of income level was declining. Furthermore, in 2002 the differentiation of this particular index showed a drastic rise vis-à-vis the prior years and reached its maximum value over the period of observations. Such a result, as well as those of other tests (Jini coefficient, Tale coefficient, 
etc.), testifies to the RF regions' failure to meet conditions of the $\sigma$-convergence hypothesis.

5. Results of the regression analysis show that the concept of an absolute (unconditional) $\beta$-convergence is accurate, as long as Russian regions are concerned. In other words, during the period in question, the regions with a 1994 lower GRP per capita rate have demonstrated higher growth rates of the respective index. The bias from results of the tests on presence of $\sigma$ convergence in this particular case can be explained by the fact that income levels of some regions (most likely, those that enjoyed the highest income level) have found themselves affected by new "shocks", rising oil prices in particular, which has dramatically boosted the oil producing regions' revenues and determined the rise in income differentiation across the whole sample, while growth rates in such regions over the period in question were likely to remain at a level not higher than those in poorer regions.

6. An additional analysis of the impact of the federal financial aid and the budget investment policy on GRP growth rates (the hypothesis of the conditional $\beta$-convergence) showed the absence of such a correlation. More than that, assessment results were likely to evidence a negative effect of both the federal and regional budget policy (or its employment for purposes other than the encouragement of economic growth) on regional growth.

7. The analysis of convergence processes showed that despite the trend to income equalization, at least, among most regions, the processes of differentiation of regions with extreme (minimummaximum) values of indicators nevertheless intensified. Accordingly, while designing an economic policy, the RF Government, the RF Ministry of Economic Development and Trade and the RF Ministry of Finance should be attentive to the fact that the focus on averaged indices is not an efficient tactic, as it 
does not allow identification of a policy optimal for each of the groups of regions. This necessitates implementation of a more effectiveness equalization (redistribution of funds) policy towards the "richest" and "poorest" Subjects of RF.

8. The present research attempted to conduct decomposition of economic growth by factors on the regional level. Given a narrow statistical base on the regional level, the authors considered various options of assessing the dynamics of the factors and conducted the assessment on the basis of the direct and dual approaches.

9. The completed assessments evidence that the unexplained percent of growth in regions' GRPs over the period concerned (1997-202) accounts for quite a huge value. Interestingly, the dynamics of the factors form the least convincing explanation of growth between 1999 and 2002, for contribution of the factors is close to zero and displays rather a drastic dispersion across regions. The examination of the first of the sub-periods concerned (1997-98) allows to explain a. 40-70\% of growth on average.

10. The reasons underlying such a low rate of the explained growth, particularly over the post-1998 sub-period and throughout the whole period in question are likely to include a low quality of statistical information on the basis of which assessments are made, on the one hand, and rigid prerequisites of the model, which may not work in the conditions of Russia's transition economy.

11. Statistical information and its comprehensiveness and quality form a critical factor, as long as the procedure of decomposition of growth is concerned. The most accurate assessments of decomposition of growth are built upon disaggregated data on output, employed production factors and their productivity. The assessments made in this paper are built on the official 
statistical information published by Rosstat. Most statistical data we use are aggregated. The information available has failed to serve as a basis for building a reliable indicator of capital costs with account of capacity loading. The employment of electricity consumption by regions for this specific purpose appears just a rough approximation that suffers a number of deficiencies discussed in this paper. The indicator of labor costs can be substantially improved, providing employment is replaced by worked hours. As earlier made computations on the nationwide level show, a change in the length of the workday forms a serious growth factor during the whole period of transition. Unfortunately, it has just recently happened that the national statistics have begun highlighting assessments of employees' work-time.

12. Proceeding from the above computations, by contrast to the value-based indicators (GRP, industry value-added, etc.) one can consider sectoral assessments that are based on the physical volume of output to be the most acceptable assessment of productivity on the regional level. The value-based indicators are vulnerable to short-term changes in pricing, to which they fail to timely adopt, which leads to biased assessments. The previous paper (IET, 2003) illustrates that this particular "price factor" proves to be quite critical.

13. According to the assessments results, the dynamics of labor productivity in industry branches vary drastically from region to region. Notably, the labor productivity in the industrial sector began to rise in 1995 on average, while the growth in output is noted only starting from 1997-99 on average. These results correspond to the nationwide assessments and highlight prognostic features of the labor productivity as they do with respect to TFP. 
14. Assessments of the dynamics of productivity can be useful for building regional development forecasts; designing mediumand longer-term regional development programs, pursuing demographic and labor policies, assessing efficacy of fiscal equalization programs and their development, as well as designing the statistical instruments on the regional level by official statistical bodies.

15. The authors can suggest conducting a statistical examination of the impact of a broad array of factors on the rise in output, basic costs and productivity as landmarks of a further analysis that should form "the second step" of the research into sources of growth. Preliminary comparisons of the assessment of labor productivity in the industrial sector with some factors evidence that the growth rate of labor productivity in the regions appears positively correlated with the volume of foreign investment and negatively correlated with financial aid to the regions.

16. Whereas capital forms one of main factors that affect labor productivity, positive correlation between productivity and investment once again proves that the growth in labor productivity was greater in the regions with relatively greater volumes of foreign investment.

17. Negative correlation between the federal financial aid and labor productivity can be determined both by the impact financial aid has on labor productivity and the inverse correlation: that is, the aid was allocated primarily to depressive regions with lower productivity growth rates, which requires an additional examination.

The Canadian record in the regional economic development area highlights the possibility for a federal center to efficiently capitalize on various, region-specific factors of regional development, such as, for instance, proximity to the USA (Ontario, 
Quebec), development of mineral resources (Alberta), creation of a "Knowledge Economy Center" (Prince Edward's Island). However, given a great role these factors play in development of individual Canadian regions,still it is the fiscal equalization policy that mattered and continue to play a critical role in liquidation of the inequality between provinces. 


\section{Bibliography}

1. Bessonov V.A. "O tochnosti svodnyh pokazatelej ekonomicheskoj dinamiki v rossijskoj perehodnoj ekonomike" $\mathrm{v}$ sb. statej "Kolichestvennye metody $\mathrm{v}$ teorii perehodnoj ekonomiki" // Ekonomika i matematicheskie metody, 2002, pp. 11 12127 (in Russian).

2. Bessonov V.A. Transformacionnyj spad i strukturnye izmeneniya $\mathrm{v}$ rossijskom promyshlennom proizvodstve. M: IEPP, 2001 (in Russian).

3. Buts B., Drobyshevsky S., Kochetkova O., Malginov G., Petrov V., Fedorov G., Kheht A., Shehovtsov A., Yudin A. Tipologiya regionov RF. - M.: SEPRA, 2002 (in Russian).

4. Granberg A., Zajtseva Ju. Valovoj regionalnyj produkt: mezhregionalnye sravneniya i dinamika. - M.: SOPS, 2003 (in Russian).

5. Dneprovskaya S., Drobyshevsky S., Izryadnova O., Levchenko D., Lederman L., Malginov G., Turuntseva M., Khobson P. Investicii: regionalnyj proekt. - M.: SEPRA, 2002 (in Russian).

6. Yu.N. Ivanov, T. A. Khomenko, "Metodologiya stiatistiki natsionalnogo bogatstava I ekonomicheskogo potentsiala", Voporsy statistiki, 10/2003.

7. Kadochnikov P., Sinelnikov S. Trunin I., Chetverikov S. Analiz pereraspredeleniya sredstv mezhdu byudzhetami subjektov Rossijskoj Federacii v ramkah sistemy mezhbjudzhetnyh otnoshenij. Ocenka svojstv stabilizacionnyh instrumentov rossijskih federalnyh vlastej. - M.: SEPRA, 2003 (in Russian).

8. Lavrovskij B., «Izmerenie regionalnoj asimmetrii na primere Rossii» // Voprosy ekonomiki, № 3, 1999, pp. 42-52 (in Russian). 
9. Mikheeva N., «Differenciaciya socialno-ekonomicheskogo polozheniya regionov Rossii i problemy regionalnoj politiki», Nauchnye doklady RPEI, №99/09, 2000 (in Russian).

10. Pchelincev O., «Rossijskij ekonomicheskij rost 1999-2000 gg. v regionalnom i globalnom kontekstah» // Problemy prognozirovaniya, № 4, 2001a, pp. 25-43 (in Russian).

11. Pchelincev O, «Regiony Rossii: sovremennoe sostoyanie i problema perekhoda k ustojchivomu razvitiyu» // Problemy prognozirovaniya, № 1, 2001b, s.102-115 (in Russian).

12. Seleznev A., «Ogranicheniya ekonomicheskogo rosta v Rossii» // Problemy prognozirovaniya, № 6, 2002, pp. 29-43 (in Russian).

13. Smorodinskaya N., Kapustin A., Malygin V., «Kaliningradskaya oblast' kak svobodnaya ekonomicheskaja zona (ocenka uslovij i rezultatov razvitiya v 1994 - 1998 gg.)» // Voprosy ekonomiki, № 9, 1999, pp. 80-107 (in Russian).

14. Entov R., Lugovoy O., Astafyeva E., Bessonov V., Voskoboynikov I., Turuntseva M., Nekipelov D., Factory ekonomicheskogo rosta rossiyskoy ekonomiki. - M.: IEPP, 2003.

15. G. Khanin, “Za vernuyu tsifru: makroekonomicheskaya statistika Rossii, khozyaystvennaya zhizn i ekonomicheskaya politika”, Voporsy statistiki, 2/2005.

16. Abramovitz M., " The Search for Sources of Growth: Areas of Ignorance, Old and New”, The Journal of Economic History, V 53, № 2. (Jun., 1993), 217-243.

17. Andersson, A., E., J. Mantsinen (1981). "Mobility of Resources, accessibility of Knowledge, and Economic Growth”, Behavioral Science, 25:5.

18. Baily M.N. (1986). "Productivity Growth and Materials Use in U.S. Manufacturing,” Quart J. Econ., V101, № 1, 185-95. 
19. Barro, R. (1998). Notes on Growth Accounting. Cambridge: NBER.

20. Barro, R., X. Sala-i-Martin (1990). "Economic growth and convergence across the United States", NBER Working paper, 3419.

21. Barro, R., X. Sala-i-Martin (2004). Economic Growth. $2^{\text {nd }}$ ed. The MIT Press.

22. Bernatrd, A.B. (1991). "Empirical implications of the convergence hypothesis”, CEPR Working paper, 23.

23. Bernatrd, A.B., S.N. Durlauf (1998). "Interpreting tests of the convergence hypothesis”, Journal of Econometrics, 71, 161173.

24. Brubaker E.R. (1968). "Embodied Technology, the Asymptotic Behavior of Capital's Age, and Soviet Growth", The Review of Economics and Statistics, V 50, № 3, 304-311.

25. Bruno M. (1982). "World Shocks, Macroeconomic Response and the Productivity Puzzle", in Slower Growth in the Western World, R.C.O. Methews, ed., London: Heinemann.

26. Castro J. (2004). "Indicators of real economic convergence. A primer”, UNU-CRIS Working papers, W-2004/2.

27. Cobb C.W., Douglas P.H. (1928). "A Theory of Production", American Economic Review. V. 18. № 1, 139-165.

28. Costello D.M. (1993). “A Cross-Country, Cross-Industry Comparison of Productivity Growth”, The Journal of Political Economy, V 101, № 2., 207-222.

29. Davis, D. R., D. E. Weinstein (2002). "Bones, Bombs, and Break Points: The Geography of Economic Activity”, American Economic Review, 92, pp. 1269-1289.

30. De Broeck M., Koen V. (2000a). "The Great Contractions in Russia, the Baltics, and the Other Countries of the Former Soviet Union: A View from the Supply Side,” IMF Working Paper 00/32. 
31. Denison, E.F. The Sources of Economic Growth in the United States and the Alternatives Before Us, Committee for Economic Development, New York, 1962.

32. Denison E.F. (1967). "Why Growth Rates Differ: Post-War Experience in Nine Western Countries”, Brookings Institution, Washington, 1967.

33. Denison E.F. (1974). “Accounting for United States Economic Growth 1929-1969”, Washington, Brookings Institution.

34. Denison E.F. (1980). "The Contribution of Capital to Economic Growth”, The American Economic Review, V. 70, № 2, Papers and Proceedings of the Ninety-Second Annual Meeting of the American Economic Association, 220-224.

35. Denison Edward F. (1978). "Capital Formation and the Recent Productivity Slowdown: Discussion”, The Journal of Finance, V 33, № 3, Papers and Proceedings of the Thirty-Sixth Annual Meeting American Finance Association, New York City December 28-30, 1977. (Jun., 1978), 1006-1010.

36. Dolinskaya I., "Explaining Russia's Output Collapse: Aggregate Sources and Regional Evidence”, IMF Institute, (Feb., 2001).

37. Ellison, G., E. Glaser (1997). "Geographic Concentration in U.S. Manufacturing Firms: a Dartboard Approach”, Journal of Political Economy, 105, pp. 889-927.

38. Esteban J.M., D.Ray (1994). "On the measurement of polarization”, Econometrica, 4, pp. 819-851.

39. Evans, P. (1996). "Using cross-country variances to evaluate growth theories", Journal of Economics Dynamics and Control, 20, pp. 1027-1049.

40. Fiani, R. (1984). "Increasing Returns, Non-Traded Inputs and Regional Development”, Economic Journal, pp. 308-323.

41. Galor, O. (1996). "Convergence? Inferences from theoretical models”, Economic Journal, 106, pp. 1056-1081. 
42. Gilbert, A., J. Gugler (1982). Cities, Poverty and Development: Urbanization in the third world". Oxford: Oxford University Press.

43. Gordon R.J. (1990). "The Measurement of Durable Goods Prices”, Chicago: University of Chicago Press.

44. Gregory R.G., Denis W.J. (1973). "Do New Embody Best Practice Technology?”, Economic Journal, V 83, 1133-1155.

45. Griliches Z. (1961). "Hedonic Price Indexes for Automobiles: An Econometric Analysis of Quality Change”, New York: NBER, 137-196.

46. Griliches Z. (1963). "The Sources of Measured Productivity Growth: United States Agriculnure, 1940-1960”, The Journal of Political Economy, V 71, № 4. (Aug., 1963), 331-346.

47. Griliches Z. (1979). "Issues in Assessing the Contribution of Research and Development to Productivity Growth”, Bell Journal of Economics, V 10, № 1, 92-116.

48. Griliches Z., Jorgenson D.W. (1967). "The Explanation of Productivity Change”, The Review of Economic Studies, V 34, № 3. (Jul., 1967), 249-283.

49. Griliches. Z., J. Mairesse. "Comparing Productivity Growth”, "European Economic Review, XXI (March/April 1983), 89119.

50. Hall R.E., “ Technical Change and Capital from the Point of View of Dual", The Review of Economic Studies, V 35, (1968), 34-46.

51. Harberger Arnold C., "A Vision of the Growth Process", The American Economic Review, V 88, № 1, (Mar., 1998), 1-32.

52. Harberger Arnold C., "Reflections on Economic Growth in Asia and the Pacific", Asian Economic Studies, V 8, 1998, 1341.

53. Harrod, R. (1948). Toward a Dynamic Economics. London 
54. Henin, P.-Y., Y. Le Pen (1995). "Les episodes de la convergence europeene”, Revue Economique, 46, p. 667-671.

55. Hirschman, A. (1958). "The Strategy of Economic Development”, Yale University Press, New Haven.

56. Holmes, T. (1999). "Localization of Industry", Review of Economics and Statistics, 81, PP. 314-333.

57. Holmes, T., J. Stevens (2002). "Geographic Concentration and Establishment Scale”, Review of Economics and Statistics, 84, pp. 682-690.

58. Hseih, Chang-Tai, "What Explains the Industrial Revolution in East Asia? Evidence fron the Factors Markets", American Economic Review, V. 54, (Jun, 2002) 502-526.

59. Hulten C.R. (1992). "Growth Accounting When Technical Change is Embodied in Capital", The American Economic Review, V 82, № 4, 964-980.

60. Jorgenson D.W. (1966). "The embodiment Hypothesis", The Journal of Political Economy, V 74, № 1, 1-17.

61. Jorgenson D.W. (1988). "Productivity and Postwar U.S. Economic Growth", The Journal of Economic Perspectives, V 2, № 4., 23-41.

62. Jorgenson D.W., Gollop F., Fraumeni B. (1987). "Productivity and U.S. Economic Growth”, Cambridge, Mass.: Harvard Univ. Press, 1987.

63. Kendrick J.W. (1956). "Productivity Trends: Capital and Labor,” Review of Economics and Statistics, V. 38. № 3, 248257.

64. Krugman, P. (1991). "Increasing returns and economic geography”, Journal of Political Economy, 99, pp. 483-499.

65. Le Pen, Y. (1998). "La convergence internationale des revenus par tête: un tour d'horizon”, Problèmes économiques, 2.558, p. 17-24. 
66. Lichtenberg, F.R. (1994). "Testing the convergence hypothesis", Review of Economics and Statistics, 76, pp. 576579.

67. Myrdal, G. (1957). Economic Theory and Underdeveloped Regions. London: Duckworth.

68. N.Kaldor, “A Model of Economic Growth”, Economic Journal, December 1957.

69. N.Kaldor, J. A. Mirrlees, "A New Model of Economic Growth”, The Review of Economic Studies, June 1962.

70. Nelson R., "Aggregate Production Functions and Medium Range Projections”, American Economic Review, V. 54, (Sep, 1964) 548-606.

71. Nijkamp, P. (1996). Handbook of Regional and Urban Economics, ed. by. Vol. 1. Elsevier Science B.V.

72. Ottaviano, G., T. Tabuchi, J.-F. Thisse (2002). “Agglomeration and trade revisited”, International Economic Review, 43, pp. 101-127.

73. Phelps E.S. (1962). "The New view of Investment: A Neoclassical Analysis”, A Quarterly Journal of Economics, V 76, 548-67.

74. Quah, D. (1992). "International patterns of growth: I. Persistence in cross-country disparities”, LSE Working paper.

75. Quah, D. (1993). "Empirical cross-section dynamics in economic growth”, European Economic Revue, 37, pp. 426434.

76. Quah, D. (1995). "International patterns of growth: II. Persistence, path dependence, and sustained take-off in growth transition", LSE Working paper.

77. Quah, D. (1996). "Twin peaks: Growth and convergence in models of distribution dynamics”, Economic Journal, 106, pp. 1045-1055. 
78. Romer, P. (1992). "Increasing Returns and New Developments in the Theory of Growth", NBER Working paper, 3098.

79. S.Kuznets, "Modern Economic Growth”, Yale, 1966.

80. Sherer F.M. (1982). "Inter-Industry Technology Flows and Productivity Growth”, The Review of Economics and Statistics V 64, 627-634.

81. Solow Robert M., "Thechnical Change and the Aggregate Production Function", The Review of Economics and Statistics V 39, № 3 (Aug., 1957), 312-320.

82. Measuring Productivity. Measurement of Aggregate and Industry-Level Productivity Growth, OECD Manual, www.SourceOECD.org, 2001.

83. Measuring Capital. Measurement of Capital Stocks, Consumption of Fixed Capital and Capital Services, OECD Manual, www.SourceOECD.org, 2001.

84. Hulten, C.H., Dean E.R., Harper M.J. (editors) New Developments in Productivity Analysis, NBER, 2001, 631 p. 
Подписано в печать 30.11.2005

Тираж 100 экз. 